\title{
Measured and Modeled Performance of a Spring Dominant Free Piston Engine Generator
}

\author{
Ramanjaneya Mehar Baba Bade \\ West Virginia University, rmb0004@mix.wvu.edu
}

Follow this and additional works at: https://researchrepository.wvu.edu/etd

Part of the Computer-Aided Engineering and Design Commons, Electro-Mechanical Systems

Commons, Energy Systems Commons, and the Heat Transfer, Combustion Commons

\section{Recommended Citation}

Bade, Ramanjaneya Mehar Baba, "Measured and Modeled Performance of a Spring Dominant Free Piston Engine Generator" (2020). Graduate Theses, Dissertations, and Problem Reports. 7528.

https://researchrepository.wvu.edu/etd/7528

This Dissertation is protected by copyright and/or related rights. It has been brought to you by the The Research Repository @ WVU with permission from the rights-holder(s). You are free to use this Dissertation in any way that is permitted by the copyright and related rights legislation that applies to your use. For other uses you must obtain permission from the rights-holder(s) directly, unless additional rights are indicated by a Creative Commons license in the record and/ or on the work itself. This Dissertation has been accepted for inclusion in WVU Graduate Theses, Dissertations, and Problem Reports collection by an authorized administrator of The Research Repository @ WVU.

For more information, please contact researchrepository@mail.wvu.edu. 


\title{
Measured and Modeled Performance of a Spring Dominant Free Piston Engine Generator
}

\section{Ramanjaneya Mehar Baba Bade}

\author{
Dissertation Submitted to the \\ Benjamin M. Statler College of Engineering and Mineral Resources \\ At West Virginia University \\ In partial fulfillment of the requirements for the degree of \\ Doctor of Philosophy in Mechanical Engineering
}

Nigel. N. Clark, Ph.D., Chair

Cosmin Dumitrescu, Ph.D.

Parviz Famouri, Ph.D.

Derek Johnson, Ph.D.

Terence Musho, Ph.D.

Department of Mechanical and Aerospace Engineering

Morgantown, West Virginia

2020

\begin{abstract}
Keywords: Free Piston, Two-stroke, Linear Engine Generator, Mechanical Springs, Resonance, Sensitivity Analysis, Control Strategy, Piston Motion Control, Cycle-to-cycle variations
\end{abstract}

Copyright@ 2020 Ramanjaneya Mehar Baba Bade 


\section{ABSTRACT \\ Measured and Modeled Performance of a Spring Dominant Free Piston Engine Generator}

\section{Ramanjaneya Mehar Baba Bade}

Free Piston Engine Generators (FPEG) directly convert the reciprocating piston motion into electricity by using a linear alternator. Unlike conventional engines with piston motion restricted by a crankshaft mechanism, the FPEG piston motion is constrained by the energy available in the system. When stiff springs are considered in the design, the FPEG system attains high frequency with high power and efficiency. The main objective of this research was to model stiff springassisted FPEG system dynamics and performance accurately, and to apply the modeling results to the development of a $1 \mathrm{~kW}$, spark ignited, natural gas fueled, FPEG experimental prototype. The experimental data was further utilized to refine and improve the existing model.

First, a MATLAB®/Simulink based multi-cycle numerical model was developed for single and dual cylinder FPEG systems to study the effects of major design parameters on FPEG dynamics and performance. When stiff springs were added, the dynamics became more sinusoidal and symmetric with respect to the initial starting position. For a total displacement of $34 \mathrm{cc}$, trapped compression ratio of 8.25 , and assumed combustion efficiency of $95 \%$, the modeled frequency and electric power varied from $72.3 \mathrm{~Hz}$ to $80.8 \mathrm{~Hz}$ and $0.81 \mathrm{~kW}$ to $0.88 \mathrm{~kW}$ for a single cylinder FPEG as the spring stiffness changed from $372 \mathrm{kN} / \mathrm{m}$ to $744 \mathrm{kN} / \mathrm{m}$. For a dual cylinder FPEG with the same conditions, these modeled values changed from $76.8 \mathrm{~Hz}$ to $84.1 \mathrm{~Hz}$ and $1.7 \mathrm{~kW}$ to 1.8 $\mathrm{kW}$ with increasing spring stiffness. The numerical model was then expanded for sensitivity studies of major design parameters. When FPEG operating conditions were considered, the effective stroke length was found to have a dominant effect on efficiency followed by compression ratio, cylinder bore, and spring stiffness respectively. The experimental FPEG prototype generating $550 \mathrm{~W}$ of electricity with indicated efficiencies exceeding $13.8 \%$ was used for model validation. 
Finally, the stable FPEG system requires a control strategy to match the power generated by the engine to the power demanded by the alternator. A model-based control strategy was developed in Stateflow ${ }^{\circledR}$ for alternator mode switching, calibration maps, energy management, ignition and fuel injection timings. With the proposed control strategy and stiff spring dominance, the modeled and experimental FPEG system maintained stable operation with cycle-to-cycle variations less than $5 \%$. 
To my father, who could not see this dissertation completed, but continues to inspire me by his dedication and example 


\section{ACKNOWLEDGEMENTS}

I thank my advisor, Dr. Nigel. N. Clark, for his support and assistance during my doctoral study at WVU. Besides my advisor, I owe my gratitude to the rest of my committee members: Dr. Parviz Famouri, Dr. Terence Musho, Dr. Derek Johnson and Dr. Cosmin Dumitrescu for their assistance, support, and vision in shaping this research document.

Special thanks go to several colleagues at WVU who contributed directly or indirectly to the work presented in this report. I thank Dr. Matthew Robinson, Mr. Jayaram Subramaniam, Ms. Fereshteh Mahmudzadeh Ghomi, Mr. Christopher Ulishney, Dr. Mahdi Darzi, Mr. Nima Zamani, Mr. Gregory Heiskell and Dr. Al Yasin for their contribution.

I acknowledge the financial and technical support from the Department of Energy's Advanced Research Projects Agency-Energy (ARPA-E). I also thank Dr. David Tew and Dr. Gokul Viswanathan for their technical guidance and support for this project.

Finally, I thank my family members and friends. As my foundation, my parents, Venkateswara Rao Bade and Kasi Annapurna Bade, offered me infinite support since I was a kid. I am fortunate to be their son and their patience and love make me feel indebted. To my sister, Priya Vani Sri, I express my gratitude for your support, love, and understanding during my doctoral study. Lastly, I also thank my best friend and mentor, Ms. PriyaankaDevi Guggilapu, without her constant encouragement, motivation, patience, and constructive criticism through the past seven years, I could not have finished this dissertation. 


\section{TABLE OF CONTENTS}

ABSTRACT

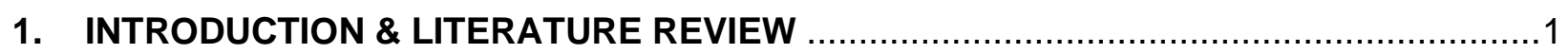

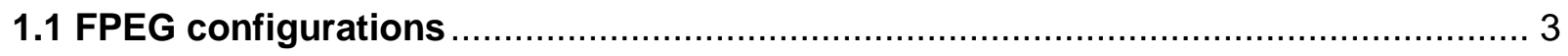

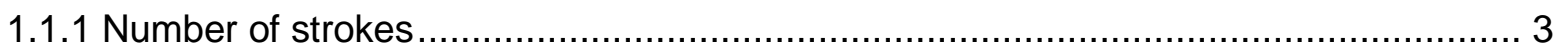

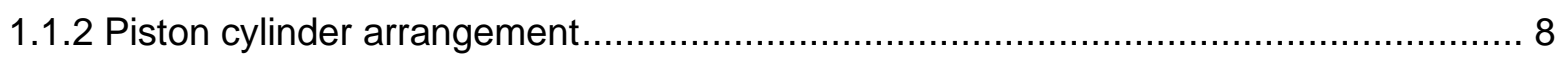

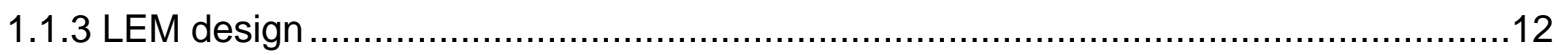

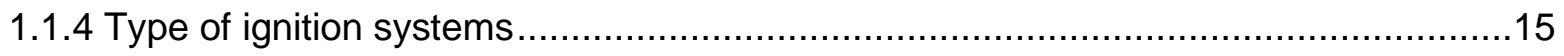

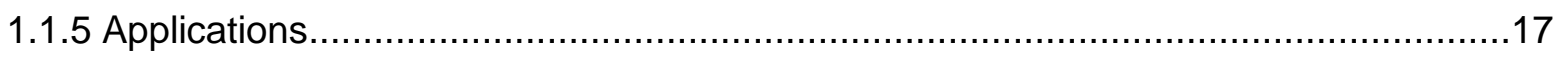

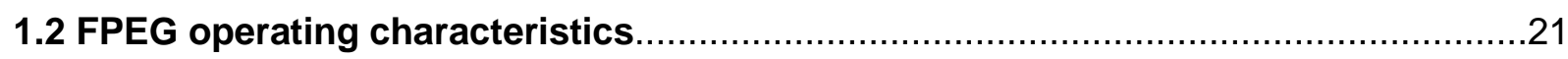

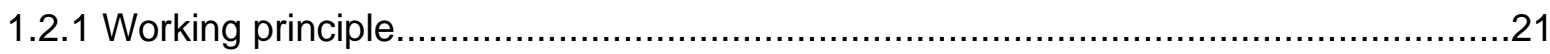

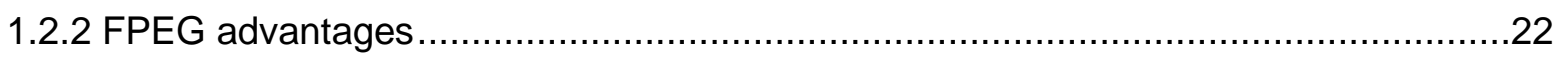

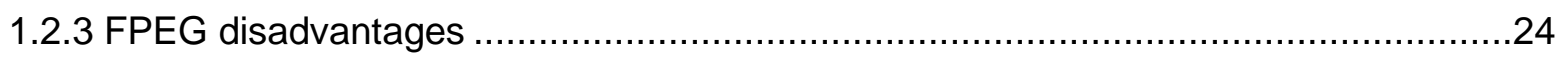

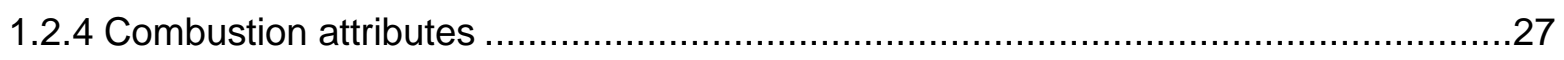

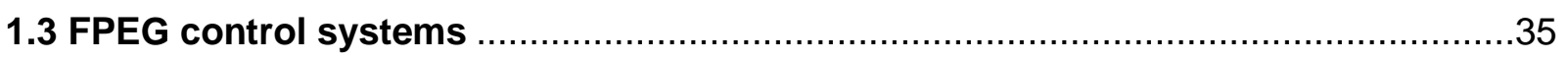

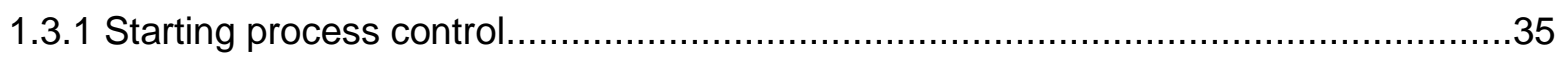

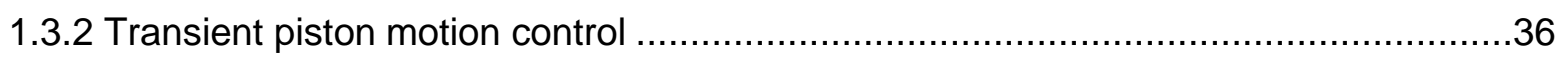

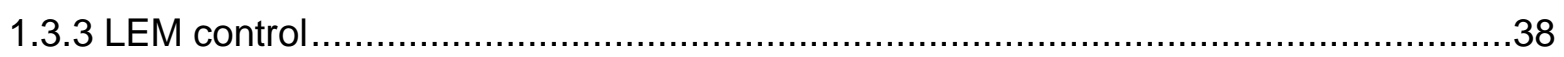

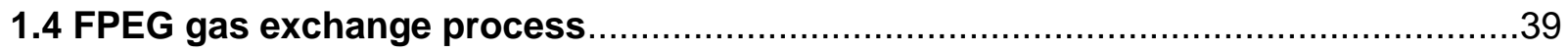

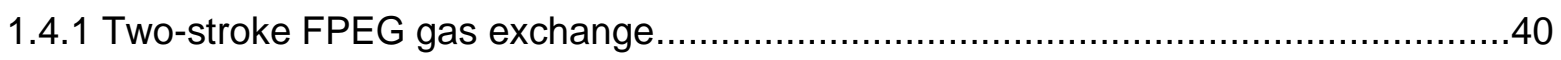

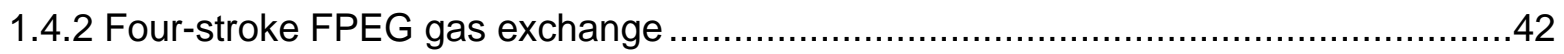

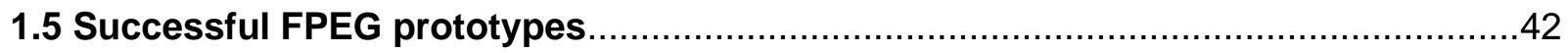

1.5.1 West Virginia University research group.....................................................43

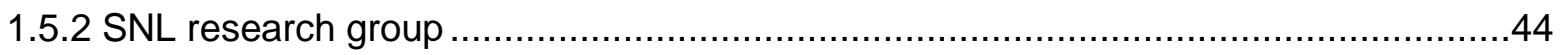




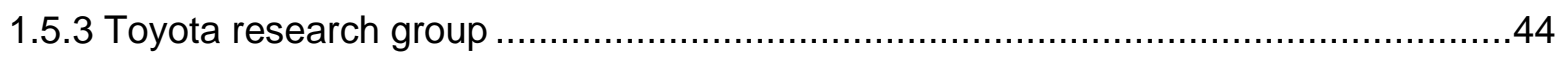

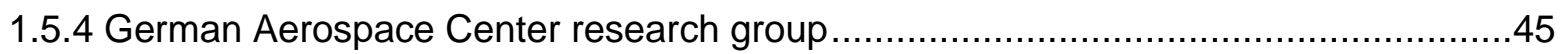

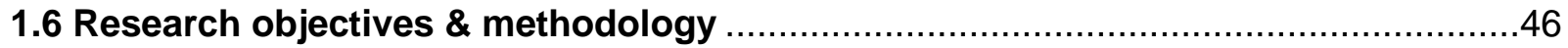

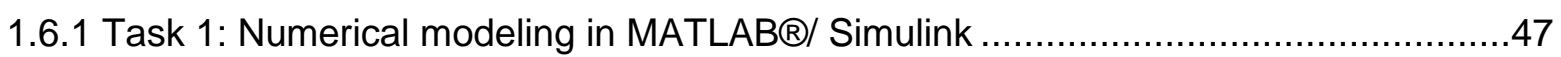

1.6.2 Task 2: Prototype Design, Testing and Model Validation ......................................47

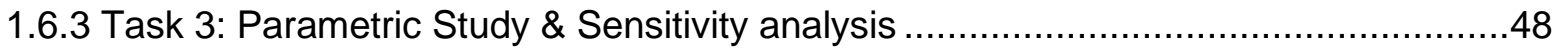

1.6.4 Task 4: Multi-level controller system development ............................................49

2. NUMERICAL MODELING IN MATLAB® / SIMULINK

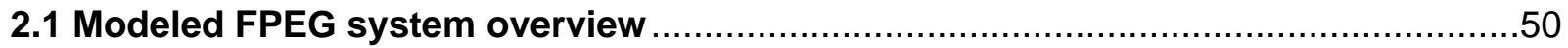

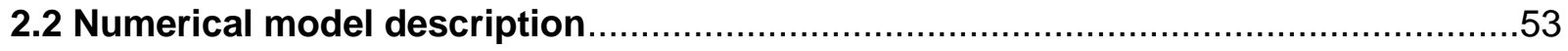

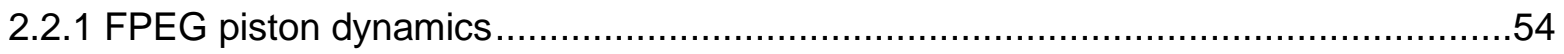

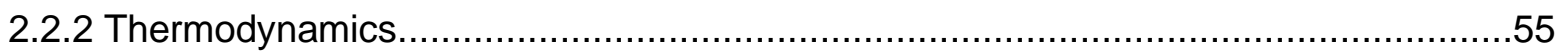

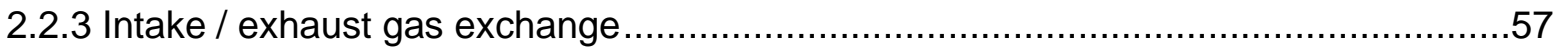

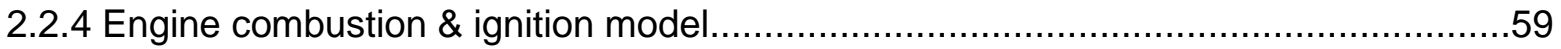

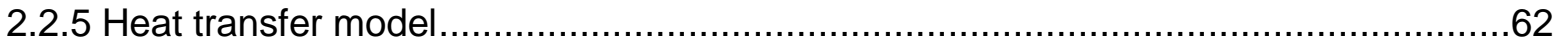

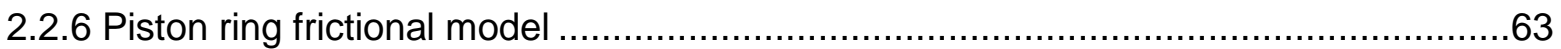

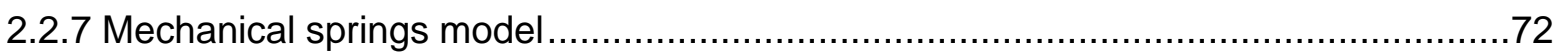

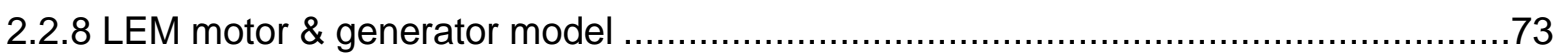

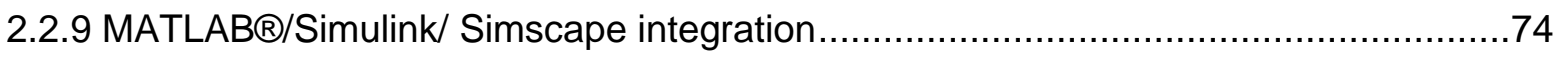

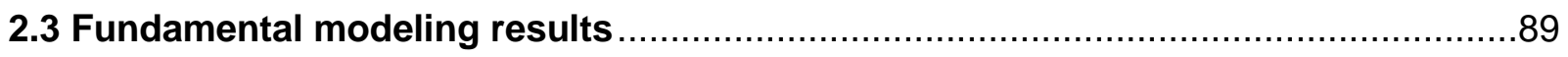

2.4 Energy analysis using the first law of thermodynamics..................................101

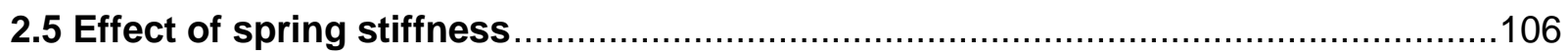

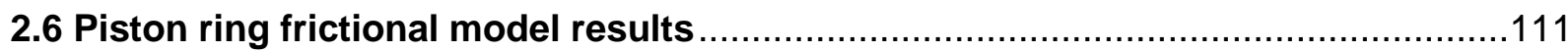

3. EXPERIMENTAL FPEG PROTOTYPE DEVELOPMENT …….............................117

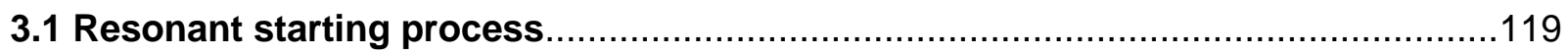

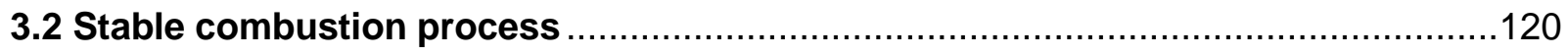

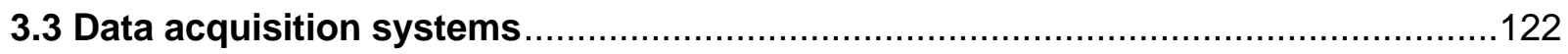

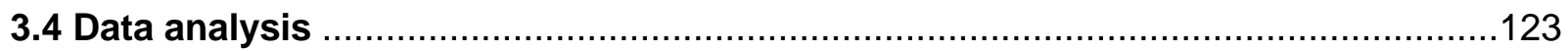




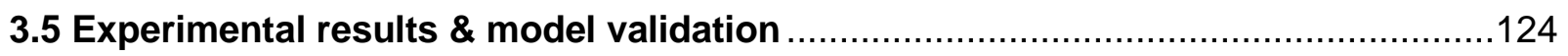

3.5.1 Resonance starting process results \& validation ...............................................125

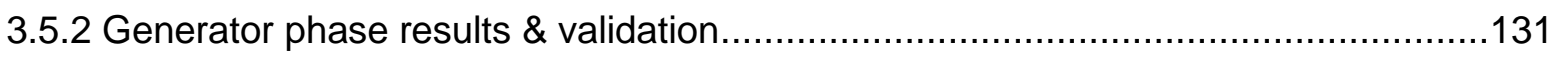

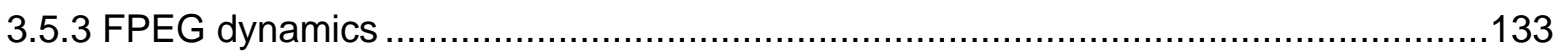

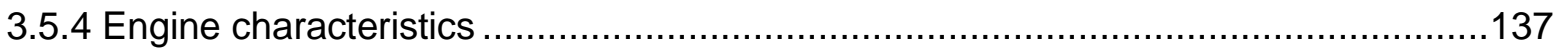

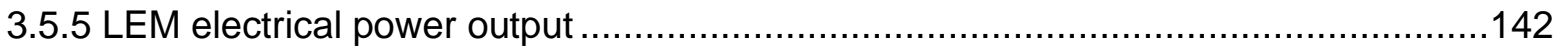

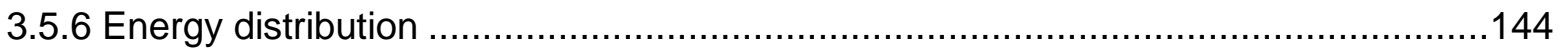

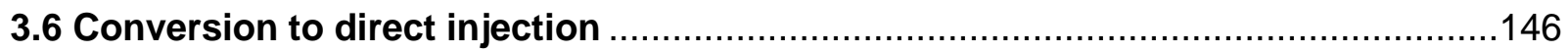

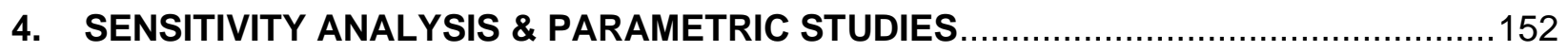

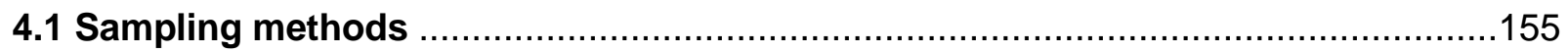

4.2 Model evaluations \& Screening methods ……................................................157

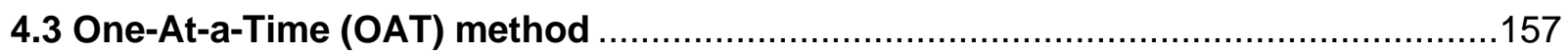

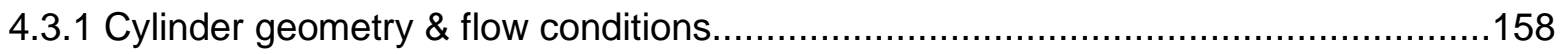

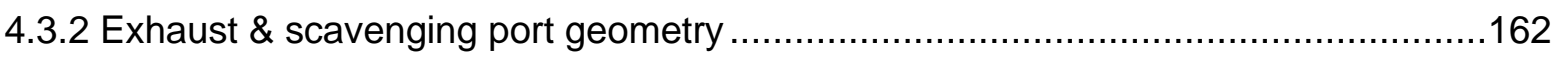

4.3.3 FPEG system design \& operating conditions ...................................................164

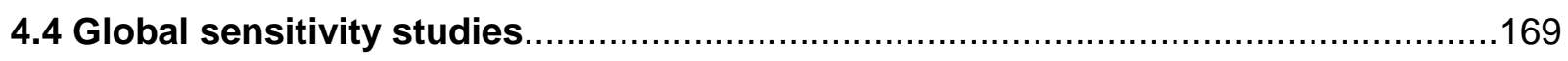

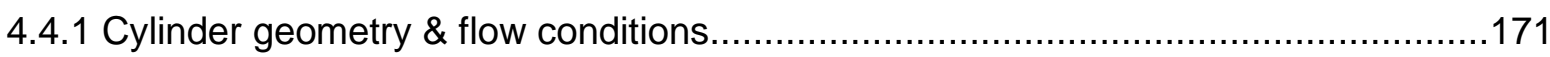

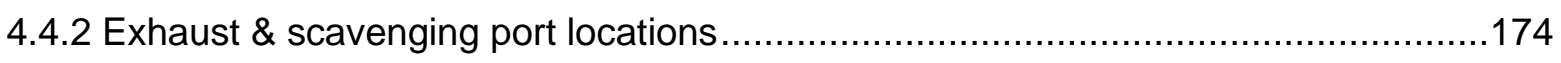

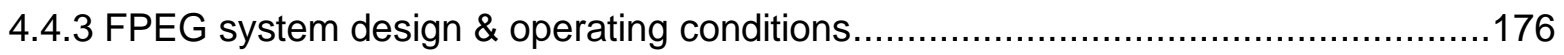

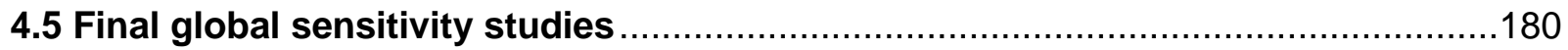

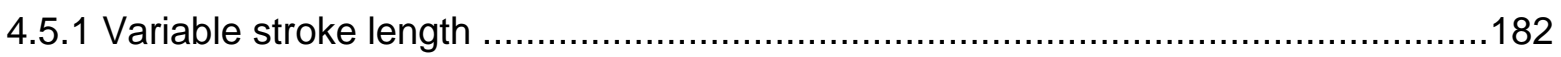

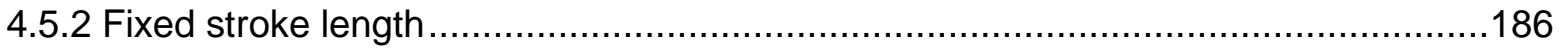

5. FPEG CONTROL ARCHITECTURE

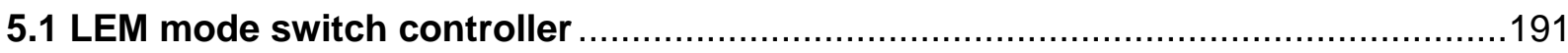

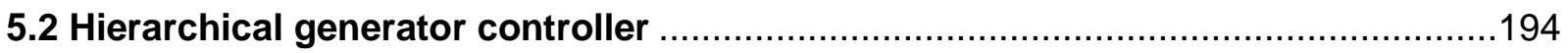

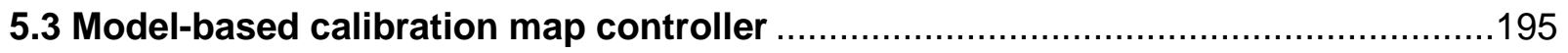

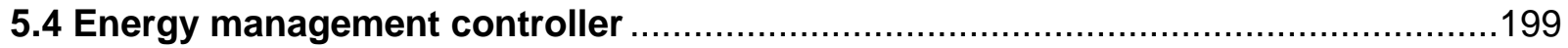

5.4.1 Energy management controller objectives.......................................................201 


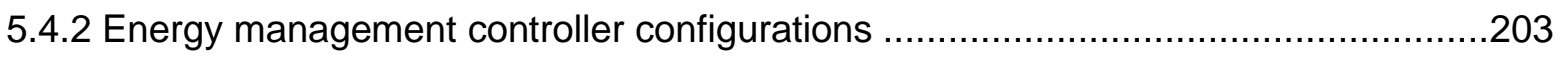

5.4.3 Controller simulation - Trapped compression ratio input ..................................205

5.4.4 Controller simulation - Dead center setpoint input ......................................208

5.4.5 Controller simulation - Peak cylinder pressure input .....................................210

5.4.6 Controller simulation - Mid-stroke velocity input.........................................213

5.4.7 Effect of model time step on controller behavior ..............................................216

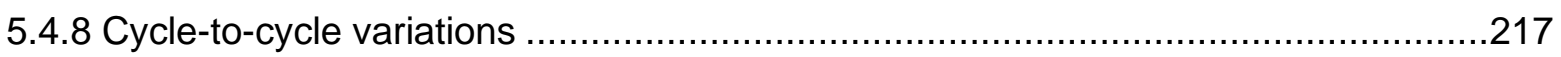

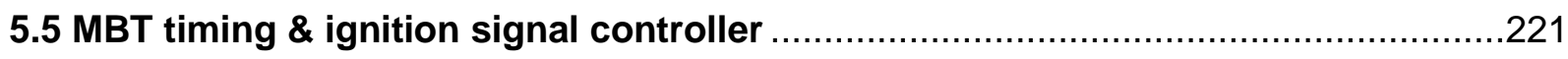

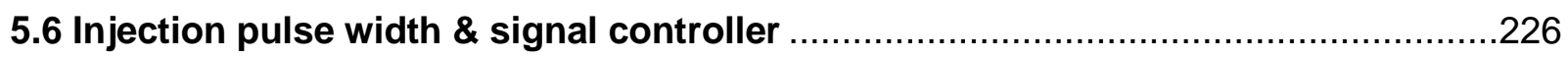

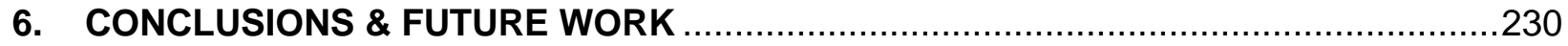

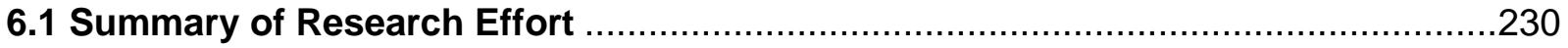

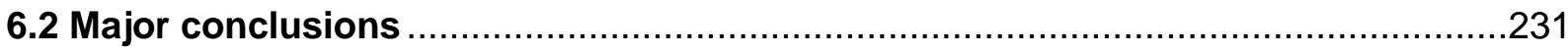

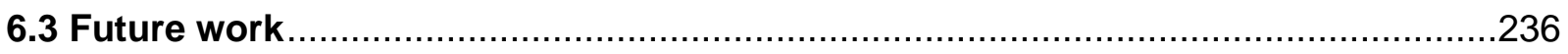

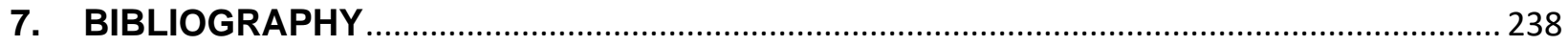




\section{LIST OF FIGURES}

Figure 1: In-cylinder pressure plotted against displacement for different intake and exhaust

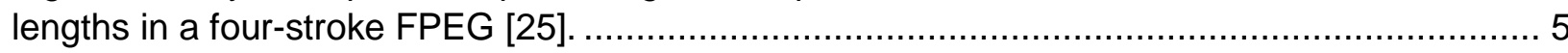
Figure 2: Comparison of two and four-stroke FPEG dynamics \& power generation characteristics [33] ......................................................................................... 7

Figure 3: Schematic diagrams of single, dual and opposed FPEG configurations [51] .............11 Figure 4: Free piston engine concepts based on applications [6, 35, 68, 73] ........................21 Figure 5: In-cylinder pressure traces and dynamics of an FPEG system during the resonance

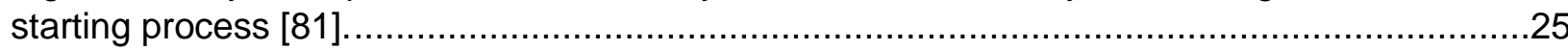
Figure 6: Pressure and mass fraction burned traces for FPEG and CSE systems of same size

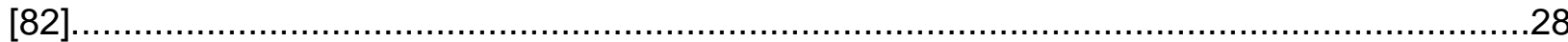
Figure 7: Gas exchange and combustion heat release comparison for a CI FPEG and CSE systems [90].... ...31

Figure 8: Charge conditions during transition from $\mathrm{SI}$ \& lean $\mathrm{SI}$ to $\mathrm{HCCl}$ mode operation in an FPEG system [62].

Figure 9: Cycle to cycle variation with trajectory piston motion control approach [45]...............37 Figure 10: Instantaneous output power comparison for a single cylinder, spring assisted FPEG

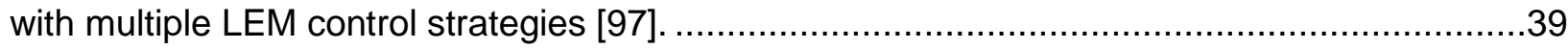
Figure 11: Cross section of a single free piston engine developed by Pempek systems [23]....42 Figure 12: Successful FPEG prototype from WVU in 1998 [34]..........................................4 Figure 13: FPEG prototype from Sandia Research Group [37] .......................................... 44

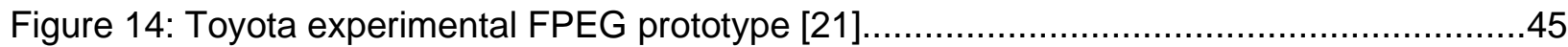
Figure 15: Experimental FPEG system from DLR [41]. .....................................................46 Figure 16: Schematic diagram and experimental prototype of a single cylinder FPEG at WVU. 51 Figure 17: Free body diagram of dual and single cylinder FPEG systems............................54 Figure 18: Schematic diagram of an FPEG cylinder domain with intake and exhaust ports.......58 Figure 19: Schematic diagram of a piston ring and cylinder liner contact in an FPEG system...65 Figure 20: Flow chart for the minimum oil film thickness calculation of piston ring in an FPEG

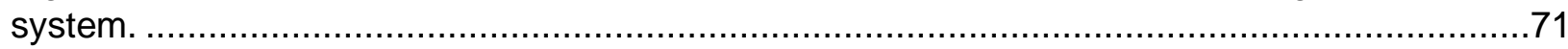

Figure 21: Equivalent circuit diagram of a LEM generator model. .......................................73 Figure 22: MATLAB®/ Simulink numerical model architecture of the FPEG system at the highest

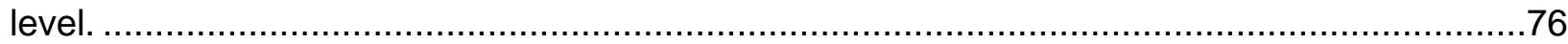

Figure 23: Architecture of FPEG plant functional block in MATLAB® / Simulink ......................77 Figure 24: Translator dynamics subsystem developed in MATLAB®/ Simulink .......................79 Figure 25: LEM motor and generator subsystem developed in MATLAB®/ Simulink .................81 Figure 26: LEM inverter circuit and motoring forces subsystem developed in Simscape. ...........82

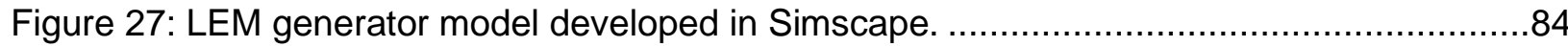
Figure 28: Cylinder pressure subsystem model developed in MATLAB®/ Simulink ..................85 Figure 29: Multiple subsystems developed inside the engine cylinder model for calculation of cylinder pressure.

Figure 30: Simplified piston ring frictional model developed in MATLAB® / Simulink for one and two piston rings. .87

Figure 31: Spring system model with damper developed in Simscape environment..................88 Figure 32: Velocity versus position plot of single FPEG, dual FPEG and CSE systems. ...........92 Figure 33: Translator acceleration and velocity distribution for CSE and FPEG systems. .........93 


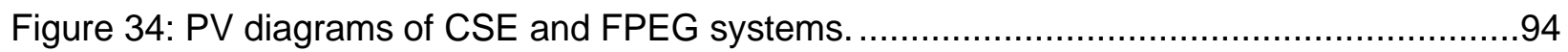

Figure 35: Region 2 of PV diagram for CSE and FPEG systems. .....................................95

Figure 36: Region 3 of PV diagram for crankshaft and FPEG systems. .................................. Figure 37: PV diagram of left and right engine cylinders in a dual FPEG system for an individual

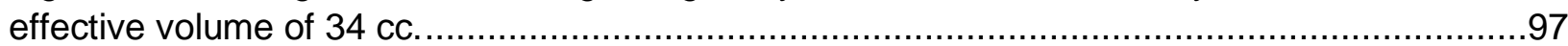

Figure 38: Distribution of forces in single and dual FPEG systems....................................98

Figure 39: Instantaneous power distribution for single and dual FPEG systems.......................99

Figure 40: Duration of gas exchange process for FPEG and crankshaft systems. .................101

Figure 41: Energy distribution of single (top) and dual (bottom) FPEG systems.....................104 Figure 42: Comparison of indicated, system and conversion losses for FPEG and CSE systems.

Figure 43: Variation in translator velocity with increasing spring systems (arrows) in single (top) and dual (bottom) cylinder FPEG systems.

Figure 44: Variation in cylinder pressure with increasing spring systems (arrows) in single (top) and dual (bottom) cylinder FPEG systems.

Figure 45: Normalized frequency and electrical power trend with increasing spring stiffness. 110 Figure 46: Comparison of minimum oil film thickness distribution for FPEG and CSE systems.

Figure 47: Comparison of piston ring frictional forces for FPEG and CSE systems.

Figure 48: Distribution of instantaneous frictional power loss due to piston ring in FPEG and CSE systems.

Figure 49: Variation in the minimum oil film thickness distribution for a single cylinder FPEG with increasing spring stiffness.

Figure 50: Variation in the piston ring frictional power losses for a single cylinder FPEG with

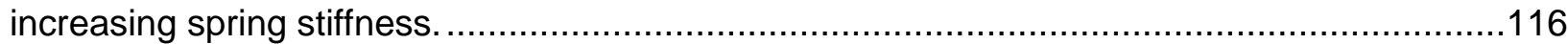

Figure 51: Experimental FPEG system under development at WVU................................118

Figure 52: Schematic diagram of prototype FPEG system at WVU...................................121

Figure 53: Translator displacement of FPEG system during the starting process..................126

Figure 54: Velocity versus position distribution of FPEG system during starting process. .......127

Figure 55: Translator velocity of FPEG system during the starting process...........................127

Figure 56: Absolute cylinder pressure of FPEG system during starting process.....................128 Figure 57: Experimental and modeled position and velocity during the FPEG starting process.

Figure 58: Experimental and modeled cylinder pressure during the FPEG starting process....131 Figure 59: Experimental and modeled velocity and position during the FPEG generating process.

Figure 60: Distribution of forces predicted by numerical model in an FPEG system. ..............134

Figure 61: Experimental and modeled dynamics during the FPEG generating process...........136 Figure 62: Deviation in the model predicted translator dynamics from experimental dynamics of the FPEG systems.

Figure 63: Crankcase pressure provided to the numerical model based on experimental data.

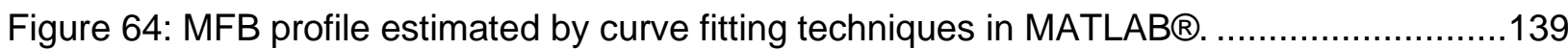

Figure 65: In-cylinder pressure distribution predicted by numerical model \& experiments. ......140

Figure 66: Deviation in the model predicted cylinder pressure from experimentally measured cylinder pressure.

Figure 67: LEM voltages and currents predicted by numerical model \& experiments. 
Figure 68: Electrical power output predicted by numerical model \& experiments. 144 Figure 69: Fuel energy distribution and indicated to electric conversion distributions of FPEG

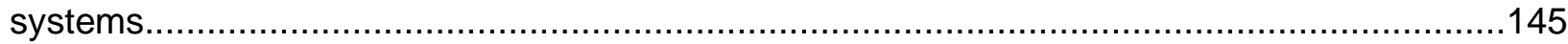

Figure 70: Translator dynamics of a direct-injected FPEG system. ...............................148

Figure 71: Cylinder pressure distribution of a direct-injected experimental FPEG system. ......149 Figure 72: Cycle-to-cycle variations in the indicated power of a direct-injected FPEG system.150 Figure 73: Variations in the energy stored in springs, LEM and energy generated by combustion in a direct-injected FPEG system.

Figure 74: Uniform probability distribution function (top) and samples generated from Sobol algorithm (bottom).

Figure 75: Variation in normalized indicated power with normalized scavenging and intake temperature.

Figure 76: Variation in normalized indicated power with normalized bore and maximum stroke length. 162

Figure 77: Variation in normalized indicated efficiency with normalized equivalence ratio, compression ratio and ignition timing.

Figure 78: Variation in the LEM power output with cylinder bore in global sensitivity analysis. 172 Figure 79: Spearman correlations for indicated power output with cylinder geometry and flow conditions.

Figure 80: Spearman correlations for FPEG system efficiency with cylinder geometry and flow conditions.

Figure 81: Spearman correlations for indicated power and FPEG system efficiency with varying port location and geometry.

Figure 82: Spearman correlations for indicated power and FPEG system efficiency with combustion heat release parameters.

Figure 83: Spearman correlations for indicated power and FPEG system efficiency with heat transfer correlation coefficients.

Figure 84: Spearman correlations for indicated power and FPEG system efficiency FPEG operating conditions.

Figure 85: Spearman correlations for indicated power and system efficiency of an FPEG system with variable stroke length.

Figure 86: Variation in the LEM power output with the stroke length.

Figure 87: Variation in the LEM power output with operating frequency.

Figure 88: Spearman partial correlation coefficients for model inputs at a constant effective stroke of $30 \mathrm{~mm}$.

Figure 89: Submodel of a multi-level controller developed in MATLABB/Simulink.

Figure 90: Submodel of a LEM inverter model developed in MATLAB®/Simulink. .................192

Figure 91: Calculation of resonant frequency based on piston reversal..............................193

Figure 92: Generating phase controller submodel developed in MATLAB®/Simulink.............195

Figure 93: Model-based calibration map of stroke length for target electrical power and

frequency.

Figure 94: Model-based calibration map of intake and exhaust pressures for target electrical power and frequency.....

Figure 95: Model-based calibration map of equivalence ratio for target electrical power and frequency.

Figure 96: Variations in peak pressure and mid-stroke velocity of the FPEG for different LEM load coefficients. 
Figure 97: Control architecture of PID \& PDF controllers modeled in MATLAB®/Simulink. .....204 Figure 98: Deviations in trapped compression ratio and TDC position with PID and PDF controllers. .206

Figure 99: Deviations in cylinder pressures and LEM power output. 206

Figure 100: PID Controller action due to step changes in the compression ratio. 207

Figure 101: Deviations in dead center positions \& compression ratio with PID and PDF controllers for dead center setpoint inputs.

Figure 102: Cycle-to-cycle cylinder pressure variation with dead center clearance set point control.

Figure 103: Cycle-to-cycle variations in compression ratio and cylinder pressures with peak cylinder pressure input.

Figure 104: Steady state in-cylinder pressure \& dead center positions with maximum pressure as an input to the controllers. 212

Figure 105: Variations in mid-stroke velocity with mid-stroke velocity control input. ..............213 Figure 106: Velocity versus position distribution during steady state with compression ratio and mid-stroke velocity input to the controllers. 214

Figure 107: Variation in the trapped compression ratio during transience and steady state operation.

Figure 108: Variation in the trapped compression ratio and dynamics with different time steps during the transient mode operation. 216

Figure 109: Stochastic variations imposed on combustion duration and combustion efficiency.

Figure 110: Variations in cylinder pressure and compression ratio with increasing spring

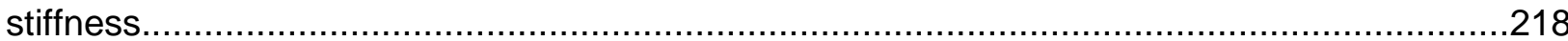

Figure 111: COV of compression ratio and peak pressures with increasing spring stiffness....219 Figure 112: Cycle-to-cycle variations in compression ratio and peak cylinder pressures with

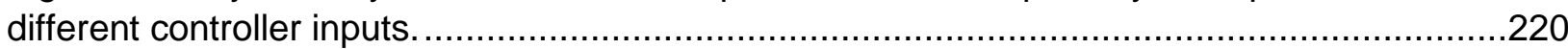

Figure 113: Distribution of MFB and its derivatives after igniting the mixture.......................222

Figure 114: Submodel developed in MATLAB®/Simulink for MBT timing calculation. .............224

Figure 115: PID controller action in regulating the 50\% MBT location and spark timing. .........225

Figure 116: Submodel for spark signal generation in MATLAB®/Simulink...........................226

Figure 117: Generation of injection signals for an FPEG system at modeling level with varying

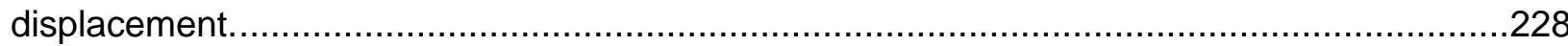

Figure 118: Injector pulse width calculation submodel based on feedback PID controllers.....229 


\section{LIST OF TABLES}

Table 1: Operating results for designs of uniflow scavenging by SNL research team [32]........41

Table 2: System specifications of the single and dual cylinder FPEG systems........................90

Table 3: Range \& fundamental values of cylinder geometry and flow inputs. 159 Table 4: Normalized LSI values for model outputs based on cylinder geometry \& flow conditions.

Table 5: Range \& fundamental values of exhaust and scavenging port geometry. 163

Table 6: Normalized LSI values for various model outputs based on exhaust and scavenging port geometry. 163

Table 7: Range \& fundamental values of FPEG system operation conditions. 165 Table 8: Normalized LSI values for various model outputs based on FPEG system operating conditions. 166

Table 9: Range \& fundamental values of parameters for final global sensitivity study. 183 


\section{ABBREVIATIONS}

\begin{tabular}{|c|c|}
\hline ARPA-E & Advanced Research Projects Agency - Energy \\
\hline BDC & Bottom Dead Center \\
\hline CFD & Computational Fluid Dynamics \\
\hline $\mathrm{CHP}$ & Combined Heat and Power \\
\hline $\mathrm{Cl}$ & Compression Ignition \\
\hline COV & Coefficient of Variation \\
\hline CR & Compression ratio \\
\hline CSE & Crankshaft Engine \\
\hline DLR & German Aerospace Center \\
\hline DOE & Department of Energy \\
\hline EGR & Exhaust Gas Retained \& Exhaust Gas Recirculation \\
\hline EHR & Exhaust Heat Rejection \\
\hline EPC & Exhaust Port Closing \\
\hline EPO & Exhaust Port Opening \\
\hline FDM & Finite Difference Method \\
\hline FEA & Finite Element Analysis \\
\hline FEM & Finite Element Method \\
\hline FPEG & Free Piston Engine Generator \\
\hline FTCS & Forward Time Central Space \\
\hline GEN & Generator \\
\hline GM & General Motors \\
\hline GSI & Global Sensitivity Index \\
\hline $\mathrm{HC}$ & Hydrocarbon \\
\hline $\mathrm{HCCl}$ & Homogenous Charge Compression Ignition \\
\hline HEV & Hybrid Electric Vehicle \\
\hline HRR & Heat Release Rate \\
\hline HT & Heat Transfer \\
\hline ICE & Internal Combustion Engine \\
\hline IGBT & Insulated Gate Bipolar Transistor \\
\hline IGN & Ignition \\
\hline IMEP & Indicated Mean Effective Pressure \\
\hline
\end{tabular}




$\begin{array}{cl}\text { INJ } & \text { Injection } \\ \text { LEM } & \text { Linear Electric Machine } \\ \text { LHV } & \text { Lower Heating Value } \\ \text { LQR } & \text { Linear Quadratic Regulator } \\ \text { LSI } & \text { Local Sensitivity Index } \\ \text { LTC } & \text { Low Temperature Combustion } \\ \text { MBT } & \text { Maximum Brake Thrust } \\ \text { MOT } & \text { Motor } \\ \text { MPV } & \text { Measured Process Variable } \\ \text { MTS } & \text { Material Testing System } \\ \text { OAT } & \text { One at a Time } \\ \text { OEM } & \text { Original Equipment Manufacturer } \\ \text { PCCI } & \text { Premixed Charge Compression Ignition } \\ \text { PDF } & \text { Pseudo Derivative Feedback } \\ \text { PID } & \text { Proportional Integral Derivative } \\ \text { PV } & \text { Pressure Volume } \\ \text { RPM } & \text { Revolutions Per Minute } \\ \text { SCFM } & \text { Standard Cubic Feet Per Minute } \\ \text { SI } & \text { Spark Ignition } \\ \text { SI } & \text { Sensitivity Index } \\ \text { SLPM } & \text { Standard Liters Per Minute } \\ \text { SNL } & \text { Sandia National Laboratory } \\ \text { SOI } & \text { Start of Injection } \\ \text { SPC } & \text { Scavenging Port Closing } \\ \text { SPO } & \text { Scavenging Port Opening } \\ \text { TDC } & \text { Top Dead Center } \\ \text { TSP } & \text { Target Set Point } \\ \text { WVU } & \text { West Virginia University } \\ \text { WVURC } & \text { West Virginia University Research Corporation } \\ & \\ \text { SPM } & \end{array}$




\section{NOMENCLATURE}

\begin{tabular}{|c|c|}
\hline$\dot{E}_{f u e l}$ & Fuel energy \\
\hline$\dot{m}_{f u e l}$ & Fuel mass flow rate \\
\hline$\dot{m}_{\text {in }}$ & Inlet mass flow rate \\
\hline$\dot{m}_{\text {out }}$ & Outlet mass flow rate \\
\hline$C_{d}$ & Discharge coefficient \\
\hline$C_{\text {load }}$ & Load Capacitance \\
\hline$H_{a}, H_{b}, H_{c}, H_{d}, H_{e}$ & Hohenberg heat transfer correlation coefficients \\
\hline$H_{\text {in }}$ & Inlet enthalpy \\
\hline$H_{\text {out }}$ & Outlet enthalpy \\
\hline$P_{L E M}$ & Electrical power output \\
\hline$P_{\text {fric }}$ & Frictional power \\
\hline$P_{\text {in }}$ & Intake pressure \\
\hline$P_{\text {ind }}$ & Indicated power \\
\hline$P_{\text {out }}$ & Exhaust pressure \\
\hline$P_{r g}$ & Interring pressure \\
\hline$Q_{\text {in }}$ & Heat addition \\
\hline$Q_{\text {out }}$ & Heat rejection \\
\hline$R_{\text {Load }}$ & Load resistance \\
\hline$W_{\text {ind }}$ & Indicated work \\
\hline$X_{b}$ & Mass fraction burned profile \\
\hline$c_{f}$ & Friction coefficient \\
\hline$c_{p}$ & Specific heat at constant volume \\
\hline$c_{v}$ & Specific heat at constant pressure \\
\hline$k_{s p r}$ & Spring stiffness \\
\hline$t_{d}$ & Combustion duration \\
\hline$t_{\text {spark }}$ & Ignition timing \\
\hline$h$ & Convective heat transfer coefficient \\
\hline$A$ & Area \\
\hline$F$ & Forces \\
\hline$H z$ & Hertz \\
\hline
\end{tabular}




\begin{tabular}{|c|c|}
\hline$L$ & Inductance \\
\hline$P$ & Pressure \\
\hline$R$ & Gas constant \\
\hline$T$ & Temperature \\
\hline$V$ & Volume \\
\hline$W$ & Watt \\
\hline$Z$ & Impedance \\
\hline$a$ & Translator acceleration \\
\hline cyl & Engine cylinder \\
\hline$f$ & Operating frequency \\
\hline$i$ & Current \\
\hline$k$ & Specific heat ratio \\
\hline$k W$ & Kilowatt \\
\hline$l$ & Left \\
\hline$m$ & Translator moving mass \\
\hline$m m$ & Millimeter \\
\hline$r$ & Right \\
\hline$S$ & seconds \\
\hline spr & Mechanical springs \\
\hline$t$ & time \\
\hline$v$ & Translator velocity \\
\hline$w$ & Work \\
\hline$x$ & Translator displacement \\
\hline$\beta$ & Damping coefficient \\
\hline$\eta$ & Efficiency \\
\hline$\mu s$ & microseconds \\
\hline$\sigma$ & Standard deviation \\
\hline$\varphi$ & Equivalence ratio \\
\hline
\end{tabular}




\section{INTRODUCTION \& LITERATURE REVIEW}

The depletion of fossil fuels and rising greenhouse gas emissions necessitate the need for highly efficient engine technology. With the development of new engine technology, tightening exhaust gas regulations pose a great challenge to the automotive engine manufacturers. While some researchers are devoted to improving and optimizing existing conventional engine technologies, other researchers are exploring and developing alternative engine technologies. A Free Piston Engine Generator (FPEG) is considered as one of the emerging technologies that meets power and consumer demands [1]. An FPEG system converts a reciprocating piston motion into electricity by using a linear alternator, with no rotational motion. A translator rod connects the engine piston to the moving component of the linear alternator. In a conventional crankshaft engine (CSE), the piston spends a relatively long time near the Top Dead Center (TDC) when compared to the FPEG system [2]. This increased residence time of the piston near TDC for the same compression ratio results in the increased heat transfer losses in a CSE system than the FPEG system. In addition to that, the frictional losses in the CSE system include piston ring friction, piston skirt friction, ring tilt, and crankshaft and connecting rod bearing friction. Since the FPEG system converts the reciprocating piston action into electricity directly, it does not require a crankshaft and connecting rod mechanism in the final design. With the elimination of crankshaft linkages, the FPEG piston motion is linear. Moreover, the losses due to piston ring tilt and skirt friction are eliminated in an ideal FPEG design. Furthermore, the frictional losses in an ideal FPEG include only the friction from the piston rings. Hence, the frictional losses are also relatively lower for an FPEG system than the CSE system [3]. Finally, the restrictions imposed by the CSE system in terms of mechanical and thermal inefficiencies are prevailed over by this novel FPEG system.

In the CSE system, a flywheel and a crankshaft-connecting rod mechanism serve as energy storage and piston motion control. Since there is no crank mechanism in the FPEG system, the piston motion is governed by the system of forces acting on a translator rod. The forces acting on the translator rod include combustion forces, frictional forces, generator forces, and rebound device or spring forces. Further, the FPEG piston motion is not constrained by the mechanical linkages when there are no springs in the design. These springs are also referred as rebound devices. The piston motion is constrained by the energy available within the FPEG system. This piston motion acts as an efficient method to control the combustion process. As a result, the FPEG system without springs exhibits variable stroke length and variable compression ratio for every 
cycle [4]. When springs were added in the final design, the cycle-to-cycle variations were reduced depending upon the spring stiffness.

Pateras Raul Pescara is credited with the modern FPEG design [5]. Pescara developed the first FPEG air compressor based on Spark Ignition (SI) combustion in 1925 and second FPEG air compressor based on Compression Ignition (Cl) combustion in 1928 [6]. At the same time, a German company Junkers also developed the free piston machinery [7, 8]. The Junkers machines were used as an air compressor in launching the torpedoes from German submarines during World War II [9]. After the success of free piston air compressors, Pescara started working on the development of the FPEG systems [10]. Since then, many researchers worked on the free piston engine concept intended for different applications. The applications include air compressors, gas generators, hydraulic engines, and electric generators. While the first three applications are used for non-road applications, the last application (electric generators) is intended for Hybrid Electric Vehicles (HEV) and Combined Heat and Power (CHP) devices. The current investigation focusses only on the FPEG system intended for HEV and CHP systems. However, the other applications are also briefly reviewed in the applications Section 1.1.5. Moreover, the contributions of this research dissertation are presented by topic wise. These topics include the numerical model, experimental prototype, and controller sections. Some of the concepts presented in one section draw on information or references from other sections presented in this document. However, these references are presented wherever possible in order to avoid confusion for the readers.

The FPEG system, in the most common geometry, consists of an Internal Combustion Engine (ICE), a Linear Electric Machine (LEM), a translator rod, and rebound devices (springs). The centrally located LEM is made of windings and permanent magnets. They are attached to either a rotor or a stator. During the start-up of the device, the LEM is operated as the motor until desired combustion conditions for the ICE are met. Just as for conventional CSE systems, the FPEG system can be made to run by using an $\mathrm{SI}, \mathrm{Cl}$, or Homogenous Charge Compression Ignition $(\mathrm{HCCl})$ combustion modes. To initiate the FPEG combustion process, the spark signal is activated for SI combustion, or the fuel is injected near the end of a compression stroke for $\mathrm{Cl}$ combustion. The engine combustion process causes the piston to reciprocate. The reciprocating piston motion produces useful electric output through the windings. Moreover, the FPEG system consists of relatively few moving parts than the CSE system. This novel design is mechanically simple and allows compact design. In some FPEG systems, a rebound device is used as a damping or energy 
storage device. This addition prevents the piston crown from hitting the head [11, 12]. Either mechanical springs or gas springs can be used for this purpose [12, 13]. Depending upon the magnitude of rebound device forces, the FPEG system can be either spring dominant or combustion dominant. In a spring dominant FPEG system, the piston motion is majorly influenced by the spring forces. For example, the addition of mechanical springs with high spring stiffness in the final design results in a nearly sinusoidal piston motion. In a combustion dominant FPEG system, the piston motion is majorly influenced by the changes in the combustion process. A single engine cylinder FPEG design requires a reversal force from the springs for its operation. Thus, the single FPEG system requires springs in the final design. The dual engine cylinder FPEG design can operate with and without the inclusion of a rebound device. The combustion dominant FPEG systems are usually dual cylinder systems. The design, operation, and the benefits of a single and a dual cylinder version are explained in Section 1.1.2.

With efficiency and cost benefits, many institutions and industries are currently exploring the use of FPEG systems. Such research institutions and industries include West Virginia University (WVU) [14], Stanford University [15], University of Texas [16], Sandia National Laboratories (SNL) [17], Newcastle University [18], Beijing Institute of Technology [19], General Motors (GM) [20], Toyota [21], FEV Engine Technology [22], and Pempek Systems Pty. Ltd. [23].

\subsection{FPEG configurations}

FPEG systems can have several different baseline configurations. In general, they are usually classified based on the number of strokes, piston cylinder arrangement, LEM design, type of combustion, and application. All of these categories are explained in a detailed manner below.

\subsubsection{Number of strokes}

Similar to conventional CSE's, the FPEG systems are classified into two-stroke engines and fourstroke engines based on the number of strokes per thermodynamic cycle. In a two-stroke FPEG system, the complete engine cycle is completed in two strokes. For every single cycle, there is a power stroke in a two-stroke single cylinder FPEG system. In a two-stroke dual cylinder FPEG system, there are two power strokes in a single cycle. In a four-stroke system, the complete engine cycle is completed in four strokes namely intake, compression, expansion, and exhaust stroke. In a four-stroke single cylinder FPEG system, the power stroke occurs once for every two 
linear cycles of operation. In a four-stroke dual cylinder FPEG system, the power stroke occurs twice for every two linear cycles of operation. As the flywheel and other linkages are eliminated in the FPEG design, the intake and exhaust valves are operated electrically or mechanically for a four-stroke design by using a position feedback signal [24]. Mechanically, these valves are operated passively through a pressure difference between the cylinder and manifolds.

\section{Four-stroke FPEG system}

The four-stroke FPEG system has improved energy savings and emission reductions when compared to a conventional CSE of the same size. This is due to the presence of more control variables to optimize the combustion process in an FPEG system [25]. These control variables include variable compression ratio, variable expansion ratio, valve overlap, variable intake, and exhaust stroke lengths. These characteristics allow for the further optimization of the existing conventional four-stroke CSE systems. However, the major disadvantage associated with the four-stroke FPEG system is the control of stringent piston motion, intake, and exhaust valve operations [24]. If the intake and exhaust valve operations are not controlled appropriately, then there is a chance for the collision of the piston crown with the valves. This collision leads to catastrophic failures and misfires, thereby, halting the continuous operation. Moreover, the LEM is operated as a motor to drive the piston during the non-power strokes in a non-spring four-stroke FPEG system [25]. As a result, a certain portion of the indicated power is lost to supply motoring power during the non-power strokes. Owing to these disadvantages, the four-stroke FPEG investigations are very few when compared to the two-stroke FPEG systems.

Xu et al. investigated the feasibility and operation of a four-stroke single cylinder FPEG system with mechanical springs in the design [25]. The mechanical springs were responsible for the compression of the cylinder contents during the non-power strokes based on the resonance theory. With mechanical springs, the motoring force was provided only for a certain percent of the stroke to overcome the damping losses. The expansion spring forces were responsible for the remaining force required for the compression of the cylinder contents. In case of an FPEG system with no springs, the LEM operated as a motor provided the motoring force throughout the nonpower strokes. Based on the simulations, a single cylinder, gasoline fueled SI FPEG system produced $2.2 \mathrm{~kW}$ electric power with $32 \%$ of fuel-to-electric conversion efficiency at an operating frequency of $25 \mathrm{~Hz}$. Moreover, a parametric investigation was carried out to understand the effect of variable intake and exhaust stroke lengths on the electric power generation. The pressure 
displacement diagrams for different intake and exhaust stroke lengths are shown in Figure 1. The results showed that long intake stroke and exhaust strokes increased the indicated work and indicated power output. As the intake stroke length was increased, more fuel was combusted when compared to the fuel combusted at a shorter intake stroke length. In case of a long exhaust stroke length, the expansion work potential was completely utilized before the opening of the exhaust valve. This allowed for expanding the mixture until the atmospheric pressure. As a result, the design followed an ideal Atkinson cycle or complete expansion cycle, which was not possible in a conventional engine. As the piston motion was fixed by the crankshaft mechanism in CSE, the cylinder pressure at the end of expansion stroke was about three to six atmospheres [25]. With an additional expansion process, the FPEG system with long exhaust stroke length achieved higher thermal efficiency and electric power than a conventional CSE for the same compression ratio.
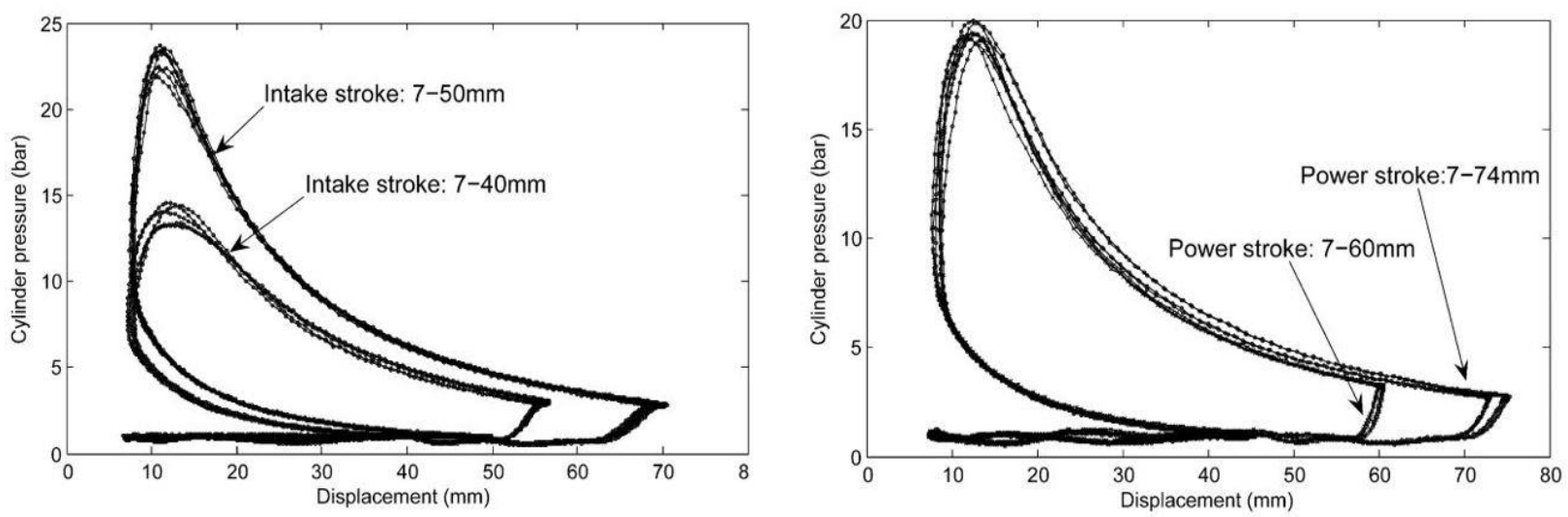

Figure 1: In-cylinder pressure plotted against displacement for different intake and exhaust lengths in a four-stroke FPEG [25].

\section{Two-stroke FPEG system}

Investigations of two-stroke FPEG systems are more common when compared to the four-stroke engines. This is due to relative simplicity in construction and operation of the two-stroke engines $[26,27,28,29,30]$. The two strokes of a usual two-stroke engine are scavenging-compression stroke and combustion-expansion stroke. The two-stroke design does not require intake and exhaust valves in the final design. The gas exchange process is achieved by using exhaust and scavenging ports. Multiple scavenging designs can be employed in a two-stroke engine depending upon the design objectives. However, the scavenging process is not perfect due to 
direct entrainment of the fuel-air mixture from scavenging port to exhaust port. This direct entrainment of the fuel mixture into the exhaust port is called short-circuiting of fuel. The magnitude of these short-circuiting losses generally depends upon the port design, the shape of the piston crown, exhaust and scavenging port pressure dynamics.

In 2002, Van Blarigan and Goldsborough from the SNL research team investigated a loopscavenged FPEG system in KIVA software. The study was aimed at the optimization of scavenging parameters for maximized performance [31]. Multiple options were analyzed and compared for loop, hybrid loop, and uniflow scavenging methods with different delivery options. Computational Fluid Dynamics (CFD) results indicated that the uniflow scavenging geometry with low intake pressure attained best engine efficiency and emission characteristics [32]. Of all the scavenging designs, the loop scavenging performance was found to be inferior. This is because of high acceleration at BDC in an FPEG system. With higher acceleration, the scavenging duration was reduced in an FPEG system when compared to a CSE system of the same stroke and frequency. This reduced time degraded the scavenging performance in a loop scavenged FPEG system. While this is true only for a dual cylinder design without springs, the loop scavenging performance in a spring dominant single cylinder design is almost similar to the CSE scavenging performance with the same stroke and frequency. The gas exchange durations for single and dual cylinder designs with stiff mechanical springs are presented in Section 2.3.

To reduce the short-circuiting losses, Pempek Systems Pty. Ltd included a special gas exchange passage, unlike a conventional two-stroke port design [23]. In their design, the exhaust gas exchange process was achieved by using an electro-pneumatic exhaust value mounted on the cylinder head. The intake procedure was achieved by using a passive intake valve. These exhaust and intake valves were mounted in such a way to achieve a uniflow scavenging design. The passive intake valve operated based on the pressure difference between the engine cylinder and compressor, and the dynamics of the mover and the gas spring force. The simulated scavenging procedure was performed within the fastest time possible, allowing for the longest expansion stroke of the combustion process.

\section{Two-stroke vs four-stroke comparison}

Many researchers investigated two-stroke and four-stroke engines individually. However, it is important to compare the performance characteristics and translator dynamics of these two 
architectures. One such numerical simulation was performed by Jia et al. [33]. They compared the in-cylinder thermodynamics and dynamics of an FPEG system operated on a two-stroke and four-stroke operation of the same prototype and initial conditions. The four-stroke and two-stroke FPEG systems were started by using the LEM as a motor without rebound devices. The simulated dynamics of the two-stroke system followed the motion of a mass-spring system. However, the four-stroke system was operated with a variable stroke duration depending upon its operation. Moreover, the piston velocity of a four-stroke engine was higher than that of a two-stroke engine during the expansion stroke. This was due to the variable intake and exhaust stroke lengths. Due to these variable conditions, the compression ratio was different for both the architectures.

The heat release process was more aligned with a constant volume process in the two-stroke engine. Moreover, the peak pressure was lower for a two-stroke than a four-stroke engine [33]. The high-pressure characteristic in a four-stroke engine was due to high mean effective compression ratio, and separation of the intake and exhaust strokes from the compression stroke. Further, the indicated and electric power outputs of a two-stroke engine were much higher than that of a four-stroke engine as shown in Figure 2.
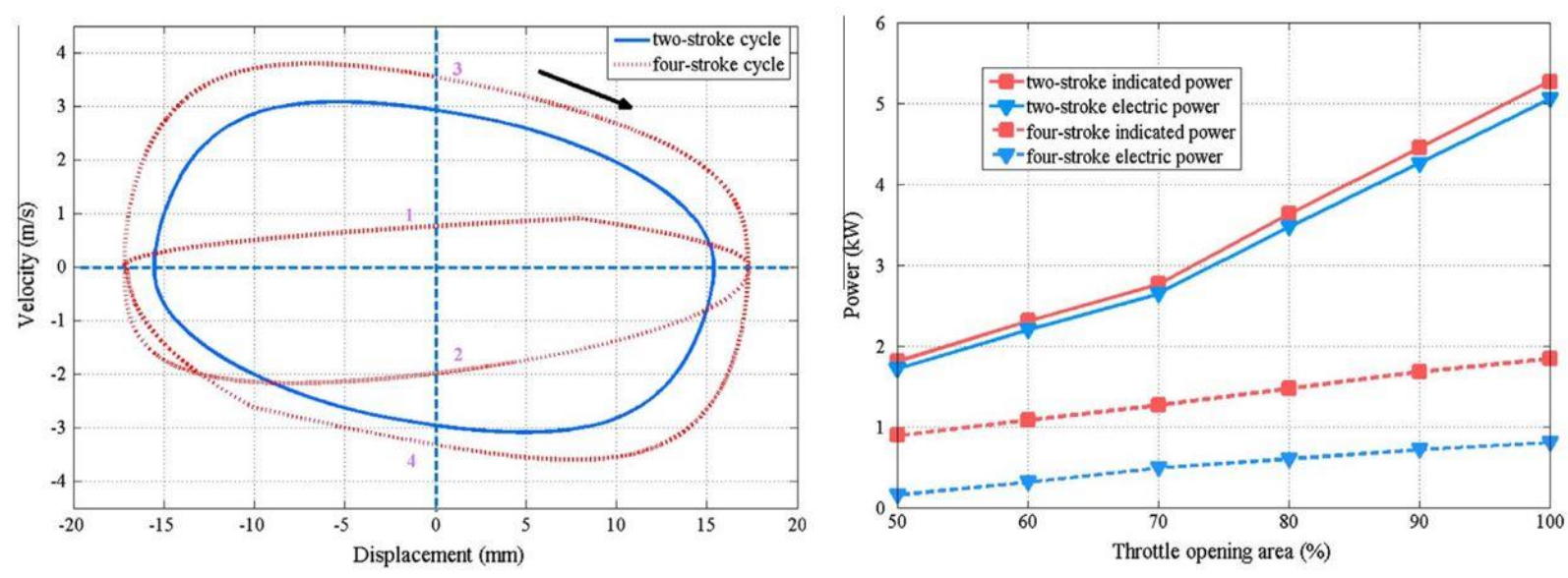

Figure 2: Comparison of two and four-stroke FPEG dynamics \& power generation characteristics [33].

From Figure 2, the difference between the indicated and electrical power was high in a four-stroke engine compared to a two-stroke engine. This high difference was due to the electrical compensation of running the LEM as a motor for non-power strokes in a four-stroke FPEG system. 
In other words, the indicated to electric conversion losses were higher in a four-stroke than a twostroke operation. As a result, the four-stroke FPEG system has a narrow operating range and lower power output than a two-stroke system for the same design geometry and input conditions.

\subsubsection{Piston cylinder arrangement}

The FPEG systems are categorized into many types based on number of engine cylinders. Of all the types, the single cylinder, dual cylinder, and opposed piston configurations are most common $[34,35,36,37,38]$. Each of these designs has their own advantages and disadvantages which are explained below.

\section{Single cylinder configuration}

A single cylinder FPEG system includes a LEM, a rebound device, and a translator rod with one ICE attached at one end. The single cylinder configuration is simple in its construction. It is also easier to control than other configurations [39]. It requires a reversal force from a LEM or a rebound device for compressing the cylinder contents during the compression stroke. The rebound device can be either a mechanical spring or a bounce chamber filled with gas. The use of a bounce chamber provides the opportunity to accurately control the energy input during the compression stroke. The energy input control variables specific to the bounce chamber are bounce chamber bore, stroke, and compression ratio. By using these variables, the bounce chamber regulates the compression ratio and stroke length.

The use of mechanical springs as a rebound device allows for a more compact and simplistic construction than the bounce chamber design [40]. However, the control ability of the system is compromised with the use of springs. In an FPEG design with mechanical springs, the researchers have no real-time control over the spring behavior. However, one can control the spring stiffness. Moreover, the spring forces vary linearly and non-linearly with stroke for mechanical springs. However, in a bounce chamber design, the force varies non-linearly with stroke. In linear mechanical springs, the losses overcome within $20 \%$ of stroke from a neutral or initial starting position. In the bounce chamber design, the same losses prevail with $50 \%$ of stroke. The neutral or initial starting position refers to the position of the piston from the cylinder head when the system is at rest. Furthermore, the addition of stiff springs in the system reduces the cycle-to-cycle variations in the FPEG system to less than $5 \%$ at the modeling level [3]. Single 
cylinder configurations with a bounce chamber and springs as rebound devices are presented in the literature [41, 42]. Moreover, the single cylinder design is not dynamically balanced. This design is more prone to vibrations when compared to other configurations. Though vibrations are a drawback, the combustion control is much easier in this configuration when compared to other configurations. This is due to the presence of only one combustion process in the system.

\section{Dual cylinder configuration}

The dual cylinder design has been the area of focus for many of the contemporary research investigations on FPEG technology [43, 44]. The dual cylinder configuration consists of a translator rod with engine cylinders mounted at each end. This design with a two-stroke operation can operate without a rebound device. However, rebound devices are required in this design when operated on a four-stroke operation. In a dual cylinder configuration without a rebound device, the boundary work from the piston in one cylinder provides the energy input for the cylinder charge compression in the other cylinder. The free piston motion without a rebound device exhibits variable stroke length and variable compression ratio every cycle due to combustion variations. The variable stroke length and compression ratio are beneficial for the optimization of the combustion process. In this design, small nuances in the combustion process of one cylinder will affect the compression ratio in the other cylinder. Further, an increase in the volumetric heat release from one cylinder will result in more energy input available for the compression stroke of another cylinder. If this energy input exceeds the maximum energy required for the compression, then it will result in the piston crown hitting the cylinder head. In contrast, if this energy input is lower than the maximum compression energy, then it will result in misfires and stalls.

The addition of a rebound device in the dual cylinder configuration stores the energy needed for the compression of cylinder contents in the other cylinder. These rebound devices reduce the impact of the piston crown hitting the head. Robinson et al. showed that the addition of mechanical springs in the dual system raised the operating frequency and power output [45]. Though dual piston configuration exhibits advantages in terms of power density and compactness, the accurate control of the combustion process in these cylinders is difficult [46]. If the combustion process is not controlled accurately, then the small variations in the current cycle will have a high impact on the compression process in the other cylinder. These combustion nuances reduced the efficiency of the FPEG system. Further, the experimental evaluations of the dual cylinder configuration without a rebound device were reported to be highly sensitive to combustion variations [47]. 


\section{Opposed piston configuration}

An opposed piston design generally consists of two pistons with a common combustion chamber. A rebound and a load device (i.e. spring and LEM) are required for each piston at the opposite ends for its operation. The opposed piston design with equal piston mass is perfectly balanced and vibration free [48]. All other configurations are dynamically unbalanced and are prone to vibrations. As both the pistons share the common combustion chamber, the heat transfer losses are reduced in this configuration. However, the major disadvantage is the accurate synchronization of two pistons. The inaccurate piston synchronization results in the reduced scavenging efficiencies and piston crowns hitting each other. In addition, the lubrication and cooling methods are complex as the common combustion chamber is located deep within the system assembly [18]. The system assembly also necessitates two sets of pistons and LEM components used in the single piston configuration. This is also a disadvantage in terms of manufacturing costs. Further, the design is more complex and bulkier than other configurations. Owing to these disadvantages, only a few opposed piston investigations have been reported in contemporary literature.

While most of the opposed piston designs were reported in the 1940's and 1960's, the only modern opposed piston investigation was reported by Hibi et al. [49]. Hibi and Ito investigated the two-stroke, direct-injected, opposed FPEG system with uniflow scavenging design as a hydraulic pump [49]. Their experimental findings reported a constant hydraulic thermal efficiency of $31 \%$ for hydraulic power output in the range of $124 \mathrm{~W}$ to $4880 \mathrm{~W}$. The schematic diagrams of single, dual, and opposed cylinder designs are presented in Figure 3 [50, 51].

\section{Single vs dual cylinder comparison}

Before proceeding with a detailed design, it is important to compare different FPEG configurations based on the piston cylinder arrangement for a variety of design variables. No such comparisons were made to date. Therefore, the author has considered the comparison of single and dual cylinder configurations with the same stroke length and operating frequency [50, 51]. At first, a single cylinder design was considered where the spring constant was varied, changing the frequency of operation and the motion of the translator. The results were then compared with the results from a dual cylinder configuration of the same stroke length and operating frequency. The 
dual cylinder displacement was twice the singe cylinder displacement. This was due to the presence of two engine cylinders of the same size used in the single cylinder design.

The simulation results showed that, without springs, the motion was far from sinusoidal, and low in frequency and power. The addition of stiff springs in the system dictated nearly sinusoidal motion and high power at high frequency. The simulation results showed that the dual cylinder configuration produced twice the power output for the same stroke length of $30 \mathrm{~mm}$ when compared to a single cylinder configuration. The detailed simulation results concerning the comparison of single and dual FPEG configurations are presented in Section 2.3 of this dissertation document.

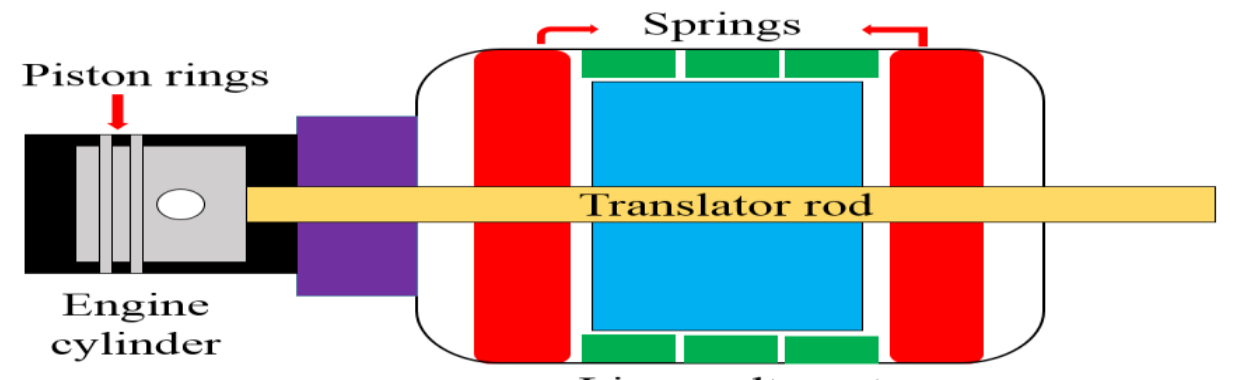

Linear alternator

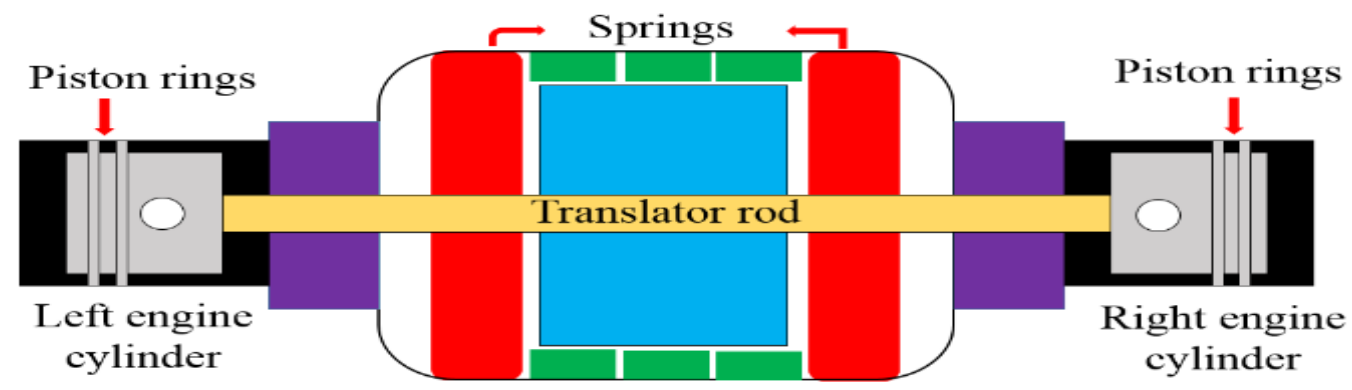

Linear alternator

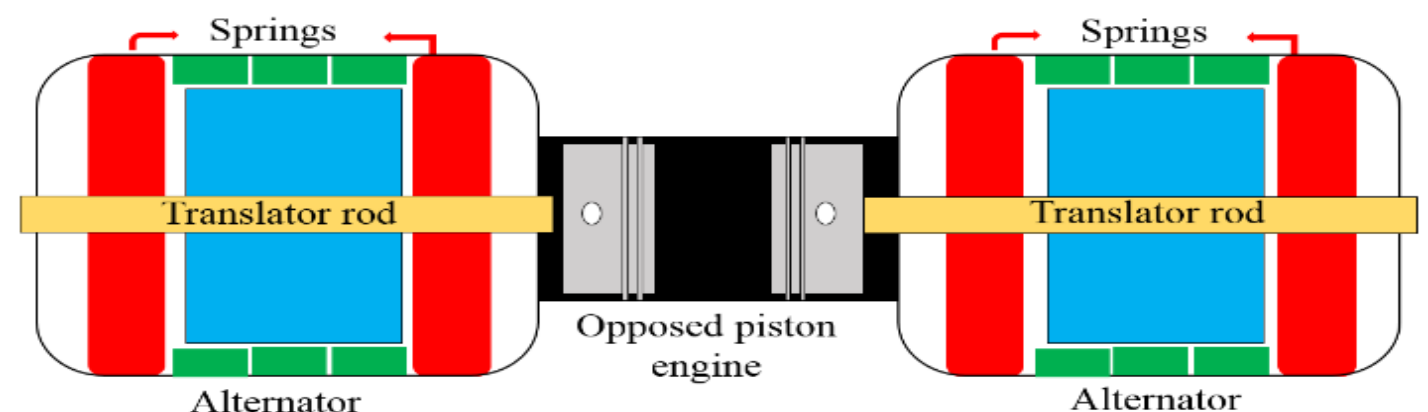

Figure 3: Schematic diagrams of single, dual and opposed FPEG configurations [51]. 


\subsubsection{LEM design}

In an FPEG system, the centrally mounted LEM can be used as both motor and a generator. During the startup of the device, the LEM is used as a motor until satisfactory ignition conditions are met. Once the combustion is initiated, the same LEM is used as a generator to produce electricity. Depending upon the LEM design, the FPEG systems are classified based on topology, phase, and magnetic cores.

\section{LEM topology}

Based on the LEM shape, the FPEG systems are classified into tubular type and flat type systems. Most of the FPEG research explorations employ tubular type because of their potential advantages over the flat type alternators.

\section{Tubular type design}

The tubular LEM contains copper windings that are mounted on a tubular stator and permanent magnets mounted on a translator rod. Hereinafter, the terms coil or windings refer to the copper windings. This design is called moving magnet design since the magnets are mounted on the mover. In addition to the moving magnet type, there is also a moving coil design. In the moving coil design, the magnets and windings are mounted on the stator and the translator rod respectively. The moving coil design requires flexible cables and is prone to vibrations and mechanical stress. As a result, the moving coil design is not favorable for high frequency applications because of its structural demerits and high moving mass [52]. Owing to these demerits, many researchers use the moving magnet design in the FPEG system.

The main benefits of moving magnet design are no end windings and zero net radial force between the stator and the translator. In this design, the translator rod has a certain freedom to rotate without changing the electrical properties of the LEM [52]. Further, the magnetic flux leakage and winding copper losses are less for this design. However, the windings in the tubular stator, laminations stacked stator, and magnetic rings are difficult to manufacture and assemble [52]. The LEM in this dissertation considered a tubular LEM with a moving magnet design at both modeling and experimental levels. 


\section{Flat type design}

The manufacturing of the flat type LEM is relatively simple than the tubular type. However, structurally it has a demerit of end-windings. For the same load, this type of design yields higher power output and efficiency when compared to a tubular design due to magnetic excitation [53]. However, the moving mass of the flat type is four times larger than the tubular type for the same output power, air gap, frequency, and number of poles [53]. This moving mass is detrimental to the operating frequency of the FPEG system. Rinderknecht et al. from the German Aerospace Center (DLR) investigated the operating characteristics of both tubular and flat type linear alternators [54]. Based on their findings, the flat type alternator was superior to the tubular type linear alternator in terms of efficiency, especially during the motoring mode. Moreover, for the FPEG systems used for HEV's, these flat type alternators can be easily integrated into the vehicle underbody. This integration lowers the center of gravity and provides more room for the crumple regions inside the engine hood [54].

\section{LEM phase}

Based on the phase, the LEM is classified into two types (single-phase and polyphase). In a polyphase system, the three-phase structure is most common. The merits and demerits of each of these types are explained below.

\section{Three-phase LEM}

In a three-phase LEM, the windings have three voltages that are generated in the windings with $120^{\circ}$ out of phase. For the same electrical power output, the size and weights of the three-phase LEM are lower when compared to a single-phase [55]. With the reduction in weight, transportation and installation become much easier. However, this design is more complex and expensive relative to a single-phase alternator. The three-phase alternator designs are used mainly for high power applications above 10kW [56].

\section{Single-phase LEM}

In a single-phase LEM, the windings have a single voltage that is generated in them. For the same power output, the single-phase LEM requires a greater number of windings than a three-phase. 
As a result, the power to weight ratio is less for this design. This kind of LEM is not very useful for higher power applications as it occupies more space than the three-phase alternator. The singlephase alternators are mostly used for FPEG applications below $10 \mathrm{~kW}$ [57].

\section{LEM magnetic core}

A magnetic core is usually defined as a magnetic material with high magnetic permeability. It guides and confines the magnetic fields in the electromechanical devices (transformers, motors, and generators). The magnetic cores are made up of ferromagnetic magnetic materials which allow the magnetic field to be concentrated within the core material. Based on the LEM magnetic core, the alternators are classified into two types, namely metallic core and air core. In a metallic core alternator, an iron core alternator is more common because of its high magnetic permeability. The merits and demerits of each of these types are explained below.

\section{Metallic core alternators}

In a metallic core alternator, a ferromagnetic magnetic material is placed between the windings to increase the magnetic field strength of the electromagnetic windings. Many ferromagnetic metals (soft iron, laminated silicon steel, special alloys, iron, and ceramics) are used as a core material. The amount of increase in the magnetic field strength depends upon the material magnetic permeability and the operating frequency. However, these metallic core materials have side effects such as eddy current losses, hysteresis, and frequency-dependent core losses. For the same level of magnetic flux, the metallic core alternators are more compact but heavier than the air core alternators.

\section{Air core alternators}

Air core alternators are the alternators without any metallic core between the electromagnetic windings. These alternators have lower inductance than the metallic core alternators of the same size. The eddy current losses and the hysteresis losses are eliminated in the air core alternators. However, for the same number of windings, the air core alternators have lower power carrying capacity than the metallic core alternators. This is due to reduced magnetic flux. An $18 \mathrm{cc}$, singlecylinder, two-stroke, natural gas fueled experimental prototype at WVU operated at a frequency 
of $72.5 \mathrm{~Hz}$ produced an average electrical power output of $130 \mathrm{~W}$ with an iron core alternator and $70 \mathrm{~W}$ with an air core alternator for the same windings and magnets.

\subsubsection{Type of ignition systems}

Similar to the conventional engines, the FPEG systems are majorly classified into three types based on the type of ignition. They include $\mathrm{SI}, \mathrm{Cl}$, and $\mathrm{HCCl}$ combustion modes. While there are other modes of ignition like Low Temperature Combustion (LTC) and Premixed Charge Compression Ignition ( $\mathrm{PCCl}$ ), the $\mathrm{SI}, \mathrm{Cl}$, and $\mathrm{HCCl}$ ignition modes are widely investigated [18, $58,59]$. The FPEG features such as variable compression ratio and variable stroke are beneficial for the $\mathrm{HCCl}$ operation, but not for the other ignition modes. In general, the stiff springs are used in the FPEG systems for reducing the cycle-to-cycle variations for conventional ignition modes [51]. Further, only the basic ignition methodologies are considered in this section. More detailed information on these ignition modes is presented in the combustion attributes section of this literature review.

\section{SI FPEG system}

In an SI FPEG system, the electrical discharge generated from the spark electrodes initiates the combustion process near TDC. The ignition timing is calculated based on the real-time displacement data measured by a linear position sensor [58]. The linear position sensor is a potentiometer in which the sliding contact shapes as a variable voltage divider. The ignition timing in an FPEG system is defined as the distance or time from BDC. This is because the crank angle has no meaning in the FPEG system. The effect of the ignition timing on the translator dynamics and overall system efficiency for different spring stiffness and operating frequencies were investigated by the author $[2,51,50]$. Two different cases were investigated. In the first case, the Maximum Brake Thrust (MBT) timing was found for different spring stiffness with the same operating frequency. In this case for all the spring stiffness, the MBT timing was found to be at the same location from BDC. Moreover, the maximum electric power output and maximum efficiency locations were coincident in this case.

In the second case, the MBT timing was found for different frequencies with the same moving mass. With increasing spring stiffness, the MBT timing was retarded due to an increase in the operating frequency. Moreover, the maximum electric power output and maximum efficiency 
locations were non-coincident in the second case. This was due to variations in the dynamics and operating frequency. In this case, the maximum power location occurred earlier than the maximum efficiency location.

The main reason for the non-coincident condition was mainly due to a change in the operating frequency. Even though constant fuel was delivered for every stroke, an increase in the frequency resulted in more strokes per unit time. These increased strokes caused more fuel delivered per unit time, thereby increasing the electrical power output. The other reason mentioned by the author was due to gas exchange variations with frequency. As a result, more/less trapped oxygen or more/less retained exhaust results in more/less fuel energy per cycle.

\section{FPEG system}

In a CI FPEG system, the combustion is initiated by the injection of fuel near TDC. The CI FPEG engines are less commonly investigated when compared to the $\mathrm{SI}$ and $\mathrm{HCCl}$ modes. This is because of device starting plus the addition and control of an external fuel injector. For SI and $\mathrm{HCCl}$ combustion modes, the fuel is supplied through the intake port. In case of a $\mathrm{Cl}$ mode, an external injector is required to supply the fuel at the end of the compression stroke. Moreover, the mistiming of the injector results in misfires and stalling of the engine. Petreanu from WVU simulated the conceptual design of a four-stroke, direct injected, $\mathrm{Cl}$ engine [59]. The simulation findings showed that the $\mathrm{Cl}$ engine operated only in a limited range. Moreover, the FPEG operation was found to be stable over a large air to fuel ratio with high indicated efficiency. Further, the study claimed an efficiency of $49 \%$ when operated on a direct injection $\mathrm{Cl}$ mode [59]. Tor et al. presented the architecture for electronic control of fuel and the effect of injection timing in a $\mathrm{Cl}$ FPEG system [29]. The injection timing control was implemented with respect to the piston position. The proposed electronic control demonstrated feasible results experimentally.

\section{HCCI FPEG system}

$\mathrm{HCCl}$ is a type of combustion in which the well-mixed fuel and air are compressed to the point of auto ignition. This alternative type of combustion achieves high efficiencies typical of $\mathrm{Cl}$ combustion and low emissions typical of SI combustion [60]. Since the ignition timing control is difficult for the FPEG systems, the $\mathrm{HCCl}$ combustion is more suitable. Most of the investigations reported in the literature are of $\mathrm{HCCl}$ combustion $[61,62,63]$. Van Blarigan et al. investigated the 
$\mathrm{HCCl}$ combustion capability in a rapid compression machine with regards to improved thermal efficiency and reduced exhaust emissions [64]. Their simulation results showed the thermal efficiency improvement by $56 \%$ than the current conventional FPEG combustion systems when operated on natural gas, propane, and hydrogen. This was due to a constant volume combustion process at a high compression ratio of $30: 1$ and the low equivalence ratio of 0.35 [64]. Besides, over compression of cylinder contents had no influence on the indicated thermal efficiency. This was due to the highest value of heat release happening before the end of the compression stroke. The above-stated conclusion was justified by the experiments. Based on the experiments, the indicated efficiency with insulated piston and cylinder head was $5 \%$ higher than the operation without the insulated coatings.

$\mathrm{Li}$ et al. investigated the performance of an FPEG system with $\mathrm{HCCl}$ combustion using Chemkin, Finite Element Method (FEM), and MATLAB®/ Simulink [65]. Based on the simulation findings, the $\mathrm{HCCl}$ combustion duration was much lower in an FPEG system than a CSE system of the same size. Further, they reported that the peak in-cylinder temperature in an FPEG system was much lower than the CSE under the same conditions. With the reduction in in-cylinder temperature, they reported that the temperature dependent emissions (i.e. $\mathrm{NO}_{x}$ emissions) were also reduced. As the compression ratio was increased, the high cylinder pressure forces reduced the time spent by the piston near TDC. As a result, the in-cylinder temperatures were reduced much faster than the CSE. Overall, the HCCI FPEG research and optimization opens a new platform for the development of advanced power sources with improved thermal efficiencies.

\subsubsection{Applications}

The FPEG concept can be used for a wide variety of applications. In general, the FPEG systems are most commonly used as air compressors, hydraulic pumps, distributed electric generators, and gas generators. The operating characteristics, advantages, disadvantages, and the reasons for commercial failure for all these applications are highlighted in this section.

\section{Free piston air compressors}

Most of the free piston air compressors including the Pescara design were a topic of interest during the period $1925-1960$ [8]. This interest led to the development of commercial prototypes at that time. The improved performance of the free piston air compressors was the main 
contributor to many research investigations between 1925 and 1960. The original free piston engine concept developed by Pescara and Junkers was an air compressor design [5, 7]. In the air compressor design, the compressor cylinders were coupled to the translating pistons in a multistage configuration. The multi-stage configuration referred to the design in which the compressed air in the clearance volume in one cylinder delivered the work to propel the next compression. As a result, the need for a rebound device was eliminated in a multi-stage design. Most of the free piston air compressors at that time were of opposed piston configuration without exception, thereby making them vibration free. The opposed piston air compressors developed by Junkers were used by the German Navy for launching torpedoes in World War II [7]. Despite its high efficiency and compactness, the free piston air compressor failed in terms of extensive commercial success. One of the reasons for such failure was due to a narrow operating range.

Beachly and Fronczak in their research paper evaluated the reasons for the commercial failure of the free piston air compressor [66]. London and Oppenheim reported the experimental operation of a free piston air compressor at a compression ratio of 40:1. Moreover, they reported problems of starting and ignition control in the experimental prototype [9]. Toutant reported that the free piston air compressor occupied $50 \%$ of the space of conventional air compressors with great advantages in submarine installations [67].

\section{Free piston gas generators}

After the success of a free piston air compressor, several research groups started working on the development of free piston gas generators. The gas generator design is similar to a free piston air compressor design except that there is no load device coupled with the engine. The hot exhaust gases from the engine are directly sent to the gas turbine. The output power is extracted from the gas turbine. A noteworthy feature of these gas generators is the low temperature exhaust gas supplied to the output turbine when compared to the conventional gas generators $[8,18]$. With this advantage, the heat transfer losses are reduced, and the gas turbine can be placed far away without any restrictions. Moreover, the synchronization mechanism in an opposed gas generator design also drives auxiliaries (water pump, fuel injection pump, and oil pump). The starting of these generators is the same as the starting of air compressors i.e. introducing the compressed air into the bounce combers. 
Large gas generators are mostly used in large scale stationary and marine power plants whereas, the small-scale gas generators are aimed at vehicle propulsion [68]. However, the intake air needs to be supercharged. An engine with a highly supercharged intake has higher mean effective pressures when compared to the conventional gas generators [68]. The advantages specific to the free piston gas generators are vibration free design, low fuel quality requirements, and low turbine material requirements.

McMullen and Ramsey claimed that the free piston gas generator was highly efficient when compared to the conventional gas turbine [69]. This was due to high compression pressures. They stated that a free piston design achieved an end-of-compression pressure of 100 times the atmospheric pressure. This value was around 6 for a conventional gas generator [69]. However, low part load efficiency, low lifetime, high failure rates, and low fuel economy resulted in the limited commercial success of these free piston gas generators.

General Motors gained attention in the free piston design after testing the GS-34 free piston generator developed by Societe Industrielle Generale de Mecanique Applicquee from France. GS-34 is one of the most successful free piston gas generators ever made. After that, they developed two gas generators, GS-14 and GMR 4-4 Hyprex. A GM-14 gas generator was installed in a US Maritime Administration vessel with unsatisfactory results. Specht evaluated the problems in the GM-14 gas generator during the testing period [70]. He reported noise, piston ring breakage, air pulsation, high maintenance costs, and difficulties in matching generators with turbines were the major problems at that time. The GMR 4-4 Hyprex was an opposed piston engine installed in a car, the XP-500 [71]. It is the first car in the world with an FPEG design. However, this engine failed to compete with the CSE at that time and was abandoned eventually [71].

\section{Free piston hydraulic pumps}

The hydraulic free piston pumps are mostly single cylinder free piston engines. These pumps have an engine cylinder mounted at one end and a hydraulic cylinder mounted at another end. The hydraulic cylinder acts as both rebound device and output load by using a hydraulic control strategy [72]. These units are aimed at off-road applications mainly earthmoving machinery and forklift trucks. These applications require high hydraulic loads from vehicle propulsion and accessories. In a conventional forklift, the hydraulic load is attained from a hydraulic pump 
powered by a conventional diesel ICE. Over conventional designs, the free piston system has the advantages of high operational flexibility, fuel economy, and excellent part load performance.

The Dutch company Innas has been carrying out research on hydraulic free piston engines for the last 20 years. The team from Innas developed a single cylinder, hydraulic free piston engine in a forklift truck [72]. Their experimental findings claimed a $20 \%$ reduction in fuel consumption at high loads and $50 \%$ at low loads. Moreover, they also reported indicated efficiencies of $50 \%$ for a power output of $17 \mathrm{~kW}$ [72]. In addition to these, they also claimed reduced emissions, lower fuel consumption, higher operational flexibility, and simple design than a conventional design.

\section{Free piston electric generator}

The FPEG system generates electrical power by using a centrally mounted LEM. Many of the contemporary research investigations focus on the development of the FPEG system. They are mainly used as either a range extender in HEV's or as a CHP device at the residential level. They can be either single cylinder, dual cylinder, or opposed cylinder configurations. These generators are compact, simple in construction, and have higher efficiencies compared to the CSE [23, 40, 51]. In a CSE system, the piston spends a relatively long time near TDC when compared to an FPEG system [2]. This increased residence time of the piston is responsible for higher heat transfer losses in a CSE system than an FPEG system. Moreover, with the elimination of crankshaft linkages, the frictional losses are relatively lower for an FPEG system [3]. However, challenges as cycle-to-cycle variations and piston motion control are reported for these engines.

Researchers from WVU were most successful within academia regarding the successful development of a running prototype. They have conducted research on FPEG systems for the past 20 years. A successful operational prototype with an output of $316 \mathrm{~W}$ was reported by WVU researchers in 1998 [1, 40, 73]. The operating characteristics, working principle, combustion, and performance characteristics of the FPEG systems are explained comprehensively in the upcoming sections. The various free piston designs used in air compressors, gas generators, hydraulic pumps, and electricity generators are shown in Figure $4[6,35,68,73]$. 


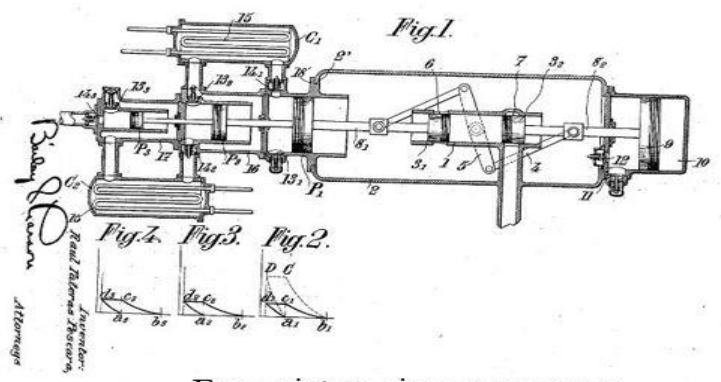

Free piston air compressor

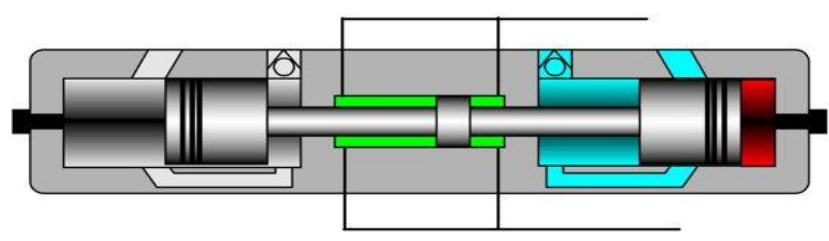

Hydraulic free piston engine

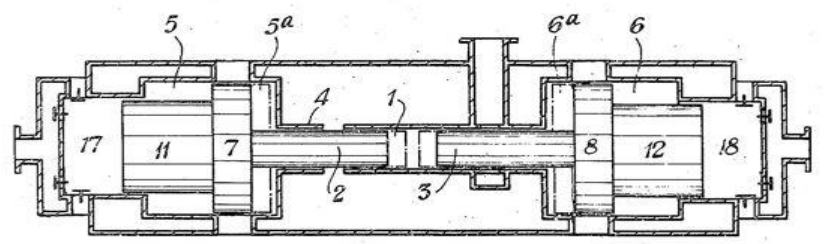

Free piston gas generator

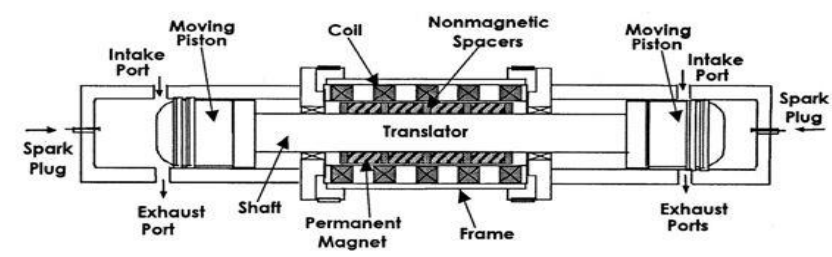

Free piston engine generator

Figure 4: Free piston engine concepts based on applications [6, 35, 68, 73].

\subsection{FPEG operating characteristics}

The FPEG systems offer many potential advantages when compared to the CSE systems of the same size and operating conditions. However, there are also some challenges associated with this technology that limited its commercial success. This section mainly deals with the advantages and the disadvantages associated with the FPEG technology. Moreover, it also highlights the issues to be addressed to make this technology commercially successful. Later, the combustion attributes of the FPEG systems are discussed comprehensively.

\subsubsection{Working principle}

In an FPEG system, the piston motion is governed by the system of forces acting on it. The mechanical work obtained from the reciprocating piston movement is converted into electricity by using a LEM. During the start-up of the device, the LEM is operated as the motor until the desired combustion conditions for ICE are met. Once the combustion is initiated, the piston reciprocates 
linearly between the dead centers. The reciprocating piston motion disturbs the magnetic flux of the LEM winding. These disturbances further produce a useful electric output from the LEM.

\subsubsection{FPEG advantages}

The section describes the advantages of the FPEG system.

\section{Structural simplicity}

The mechanical linkages that restrict the piston motion are eliminated in an FPEG system without a rebound device. With the elimination of complex mechanisms, the FPEG system exhibits simplicity in the design. In an FPEG design with rebound devices, the moving parts are higher than the FPEG design without rebound devices. This is because of the addition of the bounce chamber system or mechanical springs in the final design. Moreover, the two-stroke port scavenged FPEG design is simplistic when compared to all other configurations. Of all the configurations, opposed piston design is most complicated. This is because of the synchronization mechanism, load, and rebound devices attached to each piston.

\section{Frictional losses}

Reduction in moving parts reduces the frictional losses in an FPEG system [74, 75, 75]. Furthermore, the ideal FPEG design has no piston side forces induced by the slider crank mechanism. Jia et al. compared various friction mechanisms for the FPEG and CSE systems [74]. In a CSE system, the main friction mechanisms were identified as crankshaft and connecting rod bearing friction, piston ring, piston skirt friction, and valve train friction. In an FPEG system, the main friction mechanisms were identified as piston ring friction and LEM losses. Based on their findings, $14.4 \%$ and $8.9 \%$ of indicated power were lost to friction in the CSE and FPEG systems respectively for the same operating conditions. Of those losses, a percentage of $45 \%$ to $55 \%$ was attributed to the piston ring frictional losses. Bade et al. simulated the frictional characteristics of a piston ring in an FPEG system and compared the results with the CSE system [75]. The modeled piston ring frictional power losses were found to be $342.8 \mathrm{~W}$ and $382.5 \mathrm{~W}$ for the FPEG and CSE systems respectively [75]. In other words, the overall efficiency was improved by $0.6 \%$ in an FPEG system due to reduced piston ring frictional losses. 


\section{Multi-fuel flexibility}

The variable compression ratio in an FPEG design without a rebound device enables multi-fuel operation. A wide variety of alternative fuels is used for combustion in an FPEG system with minor hardware alterations. Flynn stated the efficient operation of an FPEG system on a wide range of fuels, including crude oil, diesel, and gasoline. Based on his experiments, he quoted that "It seems that these engines do not care whether they get fuel with octane or cetane numbers." [76]. Further, he also reported the engine operation on various vegetable and animal oils. However, the only difference was the variation in the engine power output according to the fuel heating value. Van Blarigan et al. tested eight different fuels, namely natural gas, propane, methanol, hydrogen, hexane, n-pentane, isooctane, and n-hexane on an HCCI FPEG system at a low equivalence ratio of 0.35 . His research work showed that the FPEG system attained a minimum thermal efficiency of $45 \%$ for all the fuel operations [64].

\section{Thermal efficiency}

Relative to the CSE systems, the FPEG systems attain higher thermal efficiency and higher power density. Furthermore, the short piston residence near TDC and a relatively faster expansion stroke are beneficial for improving the thermal efficiency [77]. The short residence and the fast expansion stroke of an FPEG system are also responsible for the reduction in the temperature dependent emissions. Moreover, the addition of the mechanical springs allows for the combustion process optimization for maximized indicated efficiency. This optimization is achieved due to variable piston trajectories.

\section{Vibration \& balancing}

The elimination of crankshaft mechanical linkages reduces the associated forces and moments in an FPEG system. It also eliminates the vibrations from the piston side thrust. Of all the configurations, the opposed piston configuration with equal piston masses possess minimal vibrations. Aichlmayr, in his dissertation, referred to the vibration characteristics of the Junkers free-piston air compressor at the Leipzig fair in 1936 [78].

Of all the configurations, the single cylinder designs are dynamically unbalanced with poor vibrational characteristics. The addition of a counterweight might mitigate the vibrations in this 
design. However, the design will become complex with the counterweight addition. The vibrations of the dual cylinder configuration lie in between the single cylinder and the opposed piston configuration. However, the balancing issues are yet to be addressed in the modern FPEG systems.

\section{Manufacturing \& maintenance costs}

Since the FPEG systems contain relatively fewer parts than the CSE systems, the manufacturing costs are reduced in the FPEG system [2]. The main expensive components of the FPEG system include LEM and specially designed rebound devices (if any) depending upon the system requirements.

\subsubsection{FPEG disadvantages}

This section addresses the disadvantages of the FPEG systems.

\section{Starting}

The CSE systems are started by rotating the crank for several revolutions. This is accomplished by using the rotating force of an electric motor. With the crankshaft elimination, the FPEG systems cannot be started by a rotating motor. Moreover, the FPEG designs have no mechanical couplings to receive the rotating motor force. As a result, novel methods are required for the starting of an FPEG system. Earlier in the mid-1900's, compressed air was used to start the FPEG systems [6]. Since the early designs were meant to function as an air compressor, this was not a problem. For all other applications, compressed air starting requires an auxiliary air compressor system which adds the cost and complexity to the system.

Mechanical resonance is the most common method used by the FPEG researchers to start the engine $[79,80]$. The mechanical resonance is a process in which a constant reciprocating motoring force is provided in consecutive strokes until desired combustion conditions are reached. In simple words, the resonance phenomenon refers to the process where the frequency and stroke length increase over time. In the absence of engine combustion, the engine cylinders exhibit gas spring behavior. This gas spring behavior causes the resonance phenomenon. If gas springs are used as rebound devices, the leakage and friction from the gas spring piston rings 
pose a problem during the starting. If mechanical springs are used as rebound devices, these challenges are eliminated. However, mechanical springs are subjected to damping losses. These losses are dependent upon the spring material and stiffness.

In the resonance starting method, the LEM is operated as a motor in the axial direction. Assuming a very little energy is lost during the resonance process, it is possible to apply a low motoring force. Due to the axial motoring force, the amplitudes of displacement and frequency will grow over time [81]. The displacement, velocity, and cylinder pressures for the resonance starting of the FPEG system are shown in Figure 5. From Figure 5, it is evident that the stroke length, piston velocity, and cylinder pressures are increased with time due to the resonant behavior of engine cylinders.
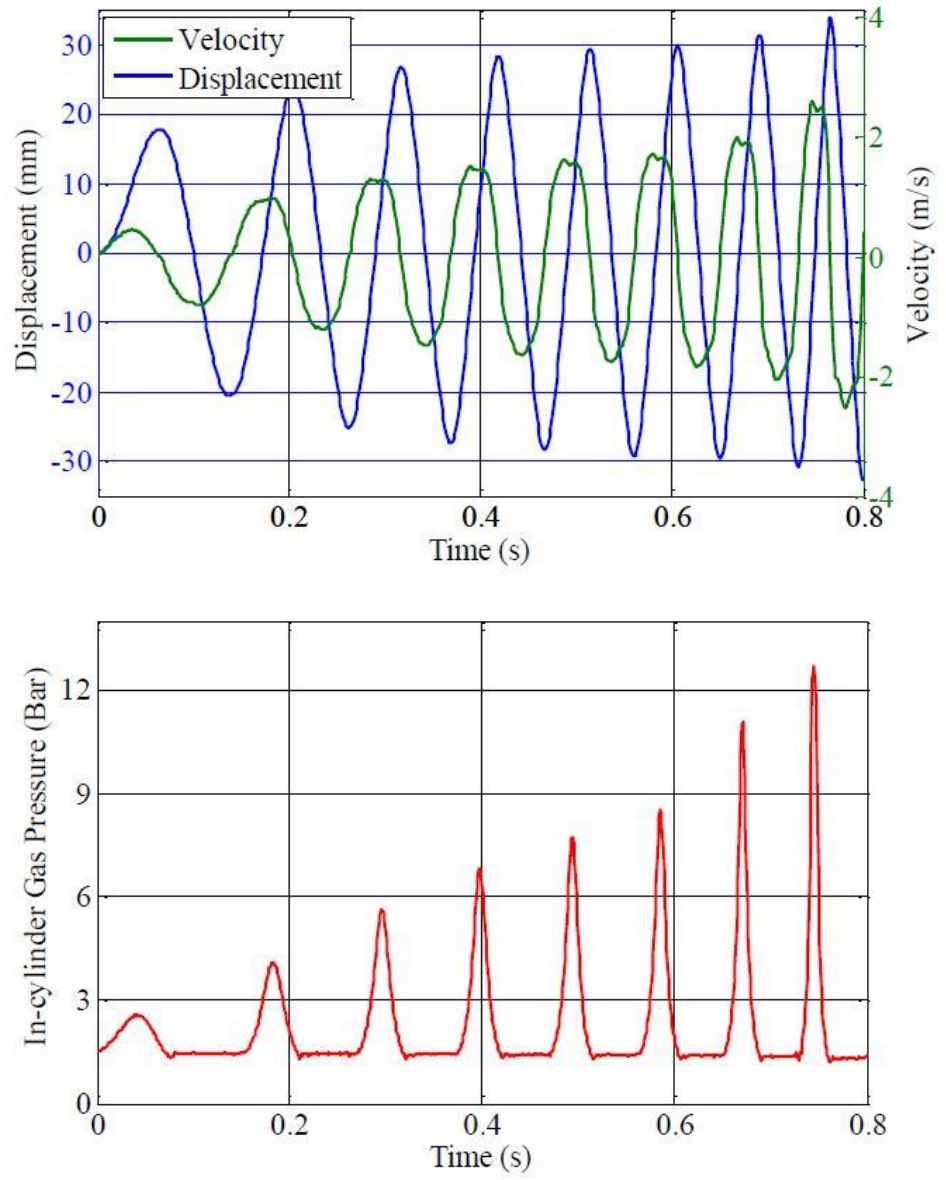

Figure 5: In-cylinder pressure traces and dynamics of an FPEG system during the resonance starting process [81]. 


\section{Device control}

With no crankshaft mechanism, the piston motion in an FPEG system is restricted by the energy available in the system. In other words, the locations of the dead centers are variable. In conventional engines, the crankshaft mechanism allows the piston to move between the fixed dead centers. In an FPEG system, this motion is governed by the system of forces acting on the translator rod. The variation in these forces causes the variations in the piston motion profile, thereby causing a variable compression ratio and variable stroke every cycle. Moreover, the piston motion is different for different operating conditions. This problem of motion control has been reported in the literature for many FPEG applications [82]. Position or piston motion control is the first and foremost important control of the FPEG design. It must be controlled within tight tolerance limits for a steady FPEG operation. The TDC position control avoids excessive combustion chamber pressures and ensures an efficient combustion process. The BDC position control enables the proper scavenging process. If the actual TDC position goes beyond the target TDC position, then there is a chance of piston crown hitting the cylinder head. Further, the engine optimization controls also depend upon proper position control. The mistiming of fuel injection and ignition results in misfires. Therefore, a robust control strategy is required for the stable operation of these systems. The FPEG control architecture is not completely explored and is evaluated in this research investigation at the modeling level.

\section{Misfires \& stalls}

Unlike conventional engines, the FPEG system without a rebound device cannot run for several cycles. This is due to the absence of an energy storage device like a flywheel in an FPEG system. In an FPEG design with springs, the springs store the energy for the compression of the cylinder contents. This energy storage allows the device to run for a few cycles without stalling. As a result, the springs in the FPEG system act like a flywheel in the CSE system. If the engine fails to build up enough compression ratio in one cycle, the translator dynamics are affected in the consecutive cycles. With the variations in dynamics, the combustion and gas exchange characteristics of an FPEG are affected, thereby resulting in misfires. Further, if other factors like ignition timing, injection timing, and valve timings (four-stroke) are not properly controlled, the engine may misfire and stop eventually. 


\subsubsection{Combustion attributes}

Similar to CSE systems, the fuel and air mixture in an FPEG can be ignited by using $\mathrm{SI}, \mathrm{Cl}$ and $\mathrm{HCCl}$ combustion modes. The FPEG system without a rebound device exhibits a variable compression ratio, which is beneficial for $\mathrm{HCCl}$ operation, but not for other combustion modes. When springs are used in conjunction with the FPEG system, the motion becomes more consistent and more sinusoidal with respect to the neutral position. Moreover, the springs also reduce cycle-to-cycle variations. For an SI combustion, a spark plug mounted on the cylinder head ignites the fuel-air mixture at the end of the compression stroke. For a $\mathrm{Cl}$ combustion, the fuel injector injects the fuel near the end of the compression stroke. The injected fuel vaporizes and mixes with the high pressure and high temperature air in the cylinder. As the temperature and pressures are beyond the ignition conditions, the fuel ignites spontaneously. In an $\mathrm{HCCl}$ combustion, a well-mixed fuel and air mixture is compressed until the point of auto-ignition. The $\mathrm{HCCl}$ combustion combines the beneficial characteristics of both $\mathrm{SI}$ and $\mathrm{Cl}$ combustions i.e. efficiency improvement and emissions reduction.

\section{Sl combustion characteristics}

In an SI combustion, the spark timing is an important control parameter that directly influences the FPEG dynamics and performance. In a CSE system, the ignition timing is described in terms of crank angle degrees before TDC. However, it may also be defined in terms of time or distance or velocity before TDC. In an FPEG system, the spark ignition timing is defined in terms of velocity or time or distance from BDC. This is due to the absence of a rotating crankshaft mechanism in an FPEG system.

Mikalsen and Roskilly investigated the performance of an SI FPEG and CSE systems by using a CFD simulation model [82]. The intake conditions and engine speed were maintained the same for both CSE and FPEG systems. Due to the fast expansion stroke, the optimal ignition timing was advanced in an FPEG system when compared to a CSE system. Based on the indicated efficiency calculations, they reported that the FPEG system had noticeable performance benefits at low speeds. With increasing the engine speed, the FPEG efficiency dropped below the CSE efficiency [82]. This was due to a relatively higher volume change in an FPEG system with increasing speeds. The pressure traces for both FPEG and CSE systems are shown in Figure 6. From Figure 6, it is evident that the FPEG system has higher in-cylinder pressure at the TDC than 
the CSE system. This is because of an advanced spark timing in an FPEG system exhibited by the dynamics. Moreover, the drop in the in-cylinder pressure after TDC was more rapid in the FPEG than the CSE system. This is due to a relatively faster expansion stroke exhibited by the FPEG system.

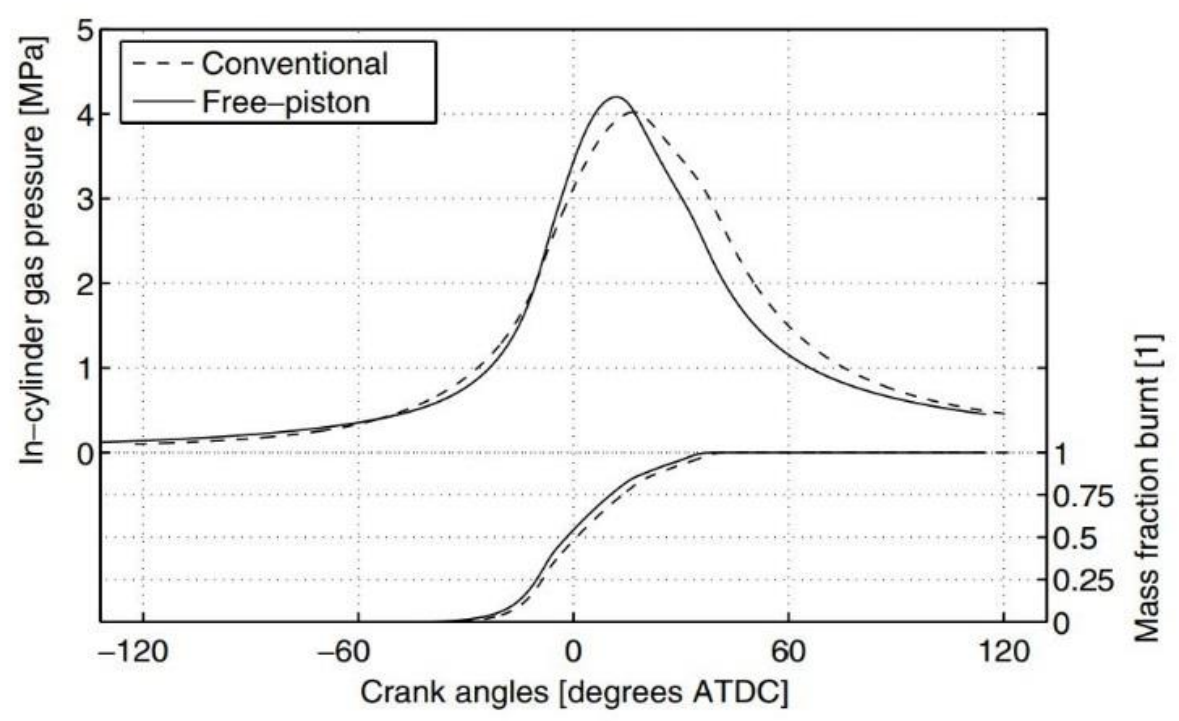

Figure 6: Pressure and mass fraction burned traces for FPEG and CSE systems of same size [82].

At an optimized ignition timing, with no Exhaust Gas Recirculation (EGR), no noticeable differences were found in the $\mathrm{CO}$ and $\mathrm{NO}_{x}$ emission levels for both systems. Moreover, the effect of spark timing on the engine-out emissions was also investigated. The efficiency penalty due to a retarded spark timing was found to be similar for both systems. However, the FPEG benefitted slightly more in terms of $\mathrm{NO}_{x}$ emissions than the CSE system with the retarded spark timing [82]. In another investigation, Baruah reported a significant reduction in $\mathrm{NO}_{x}$ emissions for a hydraulic FPEG system when the ignition timing was retarded [83]. The same conclusions were reported in the literature by many researchers $[84,85]$.

\section{Cl combustion characteristics}

Mao et al. investigated the three-dimensional combustion process of a diesel fueled FPEG by using a novel coupling technology [86]. Initially, the piston dynamics were obtained from a zerodimensional MATLAB® model. The 3D combustion and gas exchange models were defined in an AVL-FIRE CFD model. The combustion duration and ignition delay were also obtained from the 
CFD calculations. Based on these models, a novel coupling process, i.e. an iterative procedure was developed between the dynamics and combustion models. In the CFD model, a time-based two-step Wiebe function was used for the premixed and diffusion combustion. The time-based Wiebe function is similar to a conventional Wiebe function. The only exception is that the heat release rate occurs according to time rather than the crank angle degrees. From their simulation findings, the maximum temperatures and pressures were obtained at about $10^{\circ}$ equivalent crank angle degrees after TDC for an FPEG system. The equivalent crank angle degree for an FPEG system was calculated by multiplying the instantaneous cycle time with the operating frequency.

In another investigation, Kaario investigated a 3D combustion with a one-step and a two-step chemical reaction for a diesel-fueled two-stroke CI FPEG [87]. Based on the results, he reported a slight difference in the cylinder pressure distribution for these reactions. However, noticeable variations were observed for in-cylinder temperatures. The peak cylinder temperature was reduced by $100 \mathrm{~K}$ in a two-step chemical reaction during the fuel injection process. However, this variation was eliminated after the injection process. Moreover, the mass averaged gas temperature was $50 \mathrm{~K}$ less for a two-step reaction after the injection process. The main reason for this variation was due to a difference in the fuel droplet behavior. These differences were induced by the variations in the turbulent dispersion models for one-step and two-step cases.

Samhorst and Achten studied the Cl combustion characteristics in a hydraulic FPEG system [88]. The simulation results were then compared with the experimental results obtained from an Innas hydraulic FPEG system. Based on the simulation results, the major percentage of the input fuel was combusted in the premixed phase. This is due to higher piston velocities in an FPEG system than a CSE system at TDC. Moreover, it also increased the turbulence levels and gas motion in the FPEG system. As a result, a very high rate of heat release was observed in an FPEG system than the CSE system during the premixed phase. The same behavior of combustion happening predominantly in the premixed phase was reported by Tikkasen et al. [35].

Shoukry numerically simulated a two-stroke, direct-injected, CI FPEG system for a variety of design variables [89]. He investigated the effects of LEM load constant, moving mass, injection timing, and combustion duration on the FPEG system. The injection timing was defined in terms of piston position before the maximum possible stroke. The combustion process was defined by using a two-step time-based Wiebe function. The premixed and diffusion combustion ratio was provided as an input to the numerical model. Based on the numerical findings, delaying the fuel 
injection enhanced the indicated performance. Further, high in-cylinder pressures were reported with the fuel injection advance. This was due to the combustion similar to an Otto cycle combustion.

The $\mathrm{Cl}$ combustion characteristics of a diesel fueled FPEG system were also studied by Yuan et al. [90]. At first, a heat release model using a time-based Wiebe function was used to calculate the piston motion profile. With the calculated piston motion, the scavenging performance was estimated through an AVL Fire CFD model. The dynamics and scavenging results were then fed into a combustion model. Finally, the numerical simulation was repeated until the iterative convergence conditions were met. Due to variations in FPEG and CSE dynamics, these two systems had different ignition timings. Moreover, the CSE combustion time scope (i.e. $260^{\circ}$ to $460^{\circ}$ crank angle) was smaller than the FPEG combustion time scope (i.e. $244^{\circ}$ to $464^{\circ}$ crank angle). The gas exchange procedure and the combustion heat release plots for both CSE and FPEG systems are presented in Figure 7.

From Figure 7, the EGR percentage of a CSE system was smaller than that of an FPEG system operating under the same conditions. The high internal EGR content in an FPEG system was due to the reduced scavenging duration. The scavenging duration was reduced due to the relatively faster piston velocity near BDC in an FPEG system. Coming to the combustion characteristics, the positive in-cylinder pressure rising rate was higher in a CSE system than an FPEG system. With the same operating conditions, the FPEG system had minimal indicated efficiency losses. This was due to the reduced in-cylinder peak pressure and faster expansion stroke than the CSE system.

Based on Figure 7, the combustion lasted longer in an FPEG than the CSE system. Even though the combustion lasted longer, the heat release before TDC was lower for an FPEG system than the CSE system. Moreover, before TDC, the ignition delay, premixed and mixing phases of the FPEG were short. As a result, the heat release rate was less in these phases in an FPEG system. After TDC, the combustion and heat release rates were more rapid in an FPEG system. This is evident from the combustion heat release rate at $364^{\circ}$ and $365^{\circ}$ crank angles in Figure 7.

Moreover, due to the fast expansion stroke, the $\mathrm{NO}_{x}$ emissions in the FPEG system were lower when compared to a CSE system. The same trend in the $\mathrm{NO}_{x}$ emissions was reported by Mikalsen et al. in their CI FPEG system modeling [24]. Further, the mass fraction of soot and unburned 
hydrocarbon emissions had no noticeable variations in the FPEG and CSE systems [90]. In fact, these emissions were found to be slightly higher in an FPEG than the CSE system. This was due to more rapid combustion after TDC in an FPEG system than the CSE system.

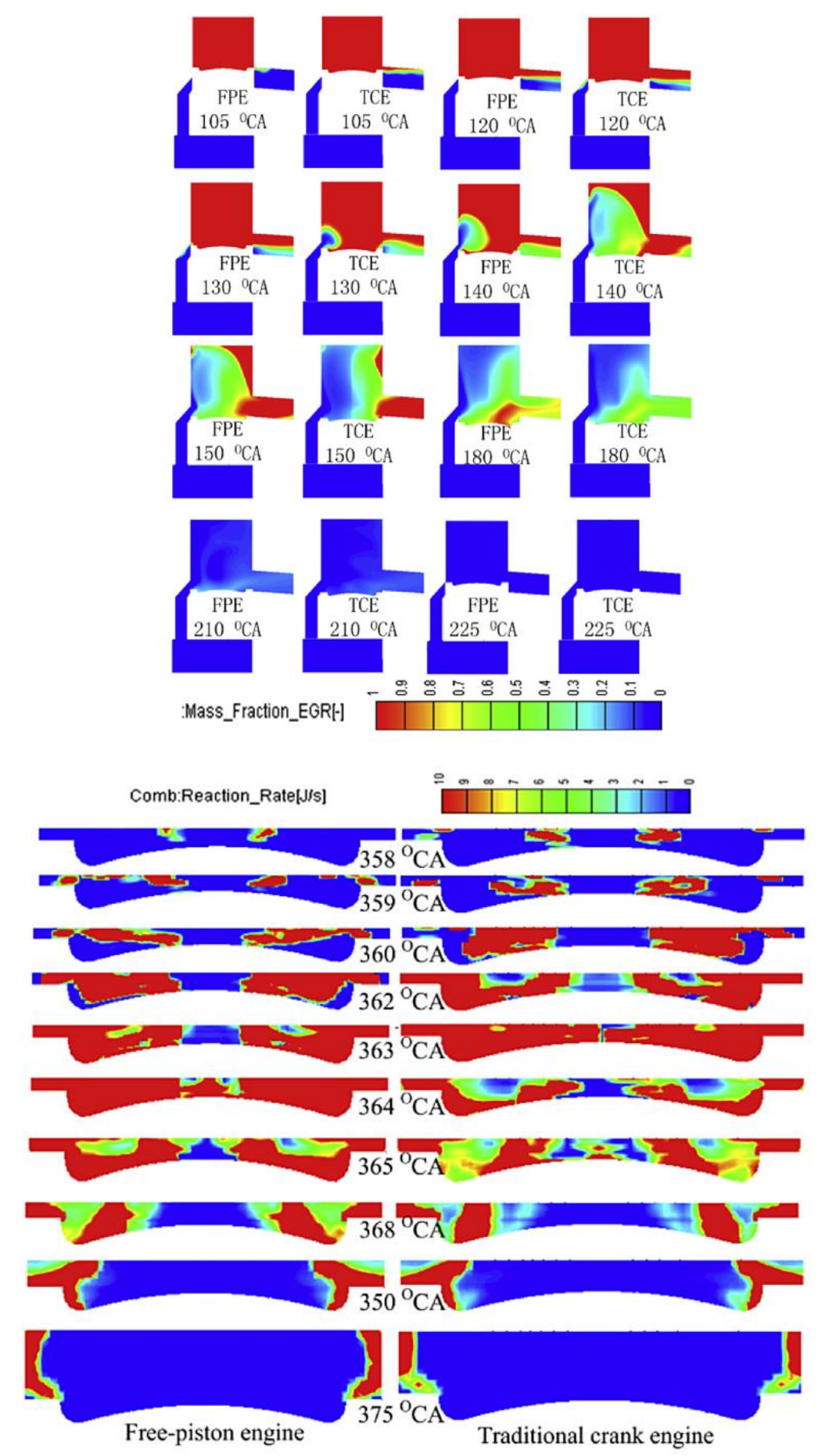

Figure 7: Gas exchange and combustion heat release comparison for a CI FPEG and CSE systems [90]. 


\section{$\mathrm{HCCl}$ combustion characteristics}

Unlike CSE systems, the FPEG exhibits variable compression ratio and variable stroke length every cycle. These unique features allow FPEG to operate on a wide variety of fuels. Many researchers investigated the use of an $\mathrm{HCCl}$ combustion in the FPEG systems [44, 62, 63]. Starting from 1998, Peter Van Blarigan from SNL worked on the ability to use $\mathrm{HCCl}$ combustion in an FPEG system [64]. The feasibility of the $\mathrm{HCCl}$ combustion in an FPEG system was investigated for eight different fuels. Propane, natural gas, hydrogen, methanol, n-pentane, hexane, n-heptane, and isooctane were the fuels used. These fuels were considered at a low equivalence ratio of $0.30-0.35$ and at an initial charge temperature of $25^{\circ} \mathrm{C}, 50^{\circ} \mathrm{C}$ and $70^{\circ} \mathrm{C}$. $\mathrm{A}$ zero-dimensional, thermodynamic model with detailed chemical kinetics, empirical scavenging, heat transfer, and friction component models was used to analyze the steady-state operating characteristics.

Experimentally, a free piston rapid compression expansion machine was used for the initial testing. Experimental findings reported a significant improvement in the indicated thermal efficiency when compared to an SI engine performance. Indicated efficiencies above $56 \%$ were achieved for the $\mathrm{HCCl}$ combustion when operated on hydrogen, propane and natural gas fuels. The primary reason for such high efficiencies was due to nearly constant volume combustion at a high compression ratio of 30:1 [44]. Furthermore, the initial charge temperature had a strong influence on the compression ratio. For the hydrogen mixture at an equivalence ratio of 0.32 , the minimum compression ratio for combustion initiation was found to be $30: 1,22: 1$ and 17:1 when the charge temperatures were $22^{\circ} \mathrm{C}, 48^{\circ} \mathrm{C}$ and $68^{\circ} \mathrm{C}$ respectively. In addition to that, the shortest combustion duration of $20 \mu$ s was recorded for any of the fuel-air mixtures tested. Furthermore, the over-compression of the fuel-air mixture had a negligible effect on thermal efficiency. This was because of the complete combustion of fuel-air mixtures earlier in the compression stroke. Further, the $\mathrm{NO}_{\mathrm{x}}$ emissions were increased with over compression. This was due to the high temperature of the fuel-air mixture. These temperatures were maintained for a relatively long time with increasing compression ratio.

Chiang et al. investigated a numerical model of SI/HCCI FPEG with electro-mechanical valves in MATLAB®/Simulink [62]. The electro-mechanical valves were responsible for the scavenging process. Moreover, these valves also maintained the proper trapped mass balance during the transition from $\mathrm{SI}$ to $\mathrm{HCCl}$ mode. Initially, the system was made to run on an SI combustion with 
high electrical power demand. Later, the combustion mode was switched to an $\mathrm{HCCl}$ combustion. Initially, three different operating modes were investigated, i.e. stoichiometric SI, lean burn SI and ultra-lean $\mathrm{HCCl}$ combustion modes. Based on the simulation results, the stoichiometric SI combustion mode produced a high electric power output of $9.2 \mathrm{~kW}$ at a low indicated efficiency of $29 \%$. When the same engine was operated on the $\mathrm{HCCl}$ mode, the power output decreased to $5.7 \mathrm{~kW}$, whereas the indicated efficiency increased to $56.3 \%$. In terms of indicated efficiencies, the $\mathrm{HCCl}$ combustion mode was beneficial for FPEG systems.

Later, they also investigated two different cases for the transition from SI to HCCI mode. In the first case, the direct transition was demonstrated from the stoichiometric SI combustion to $\mathrm{HCCl}$ combustion. In the second case, the transition was demonstrated from the lean burn SI combustion at a medium power level to $\mathrm{HCCl}$ combustion. The direct transition was carried out by turning off the spark, reducing the LEM electromagnetic force and injecting less fuel to maintain ultra-lean conditions. The simulation results reported misfires in the first case (as shown in Figure 8) due to improper valve timings and fuel control. From Figure 8 , it is evident that the piston motion is slowed down in cycle 4 due to improper control.
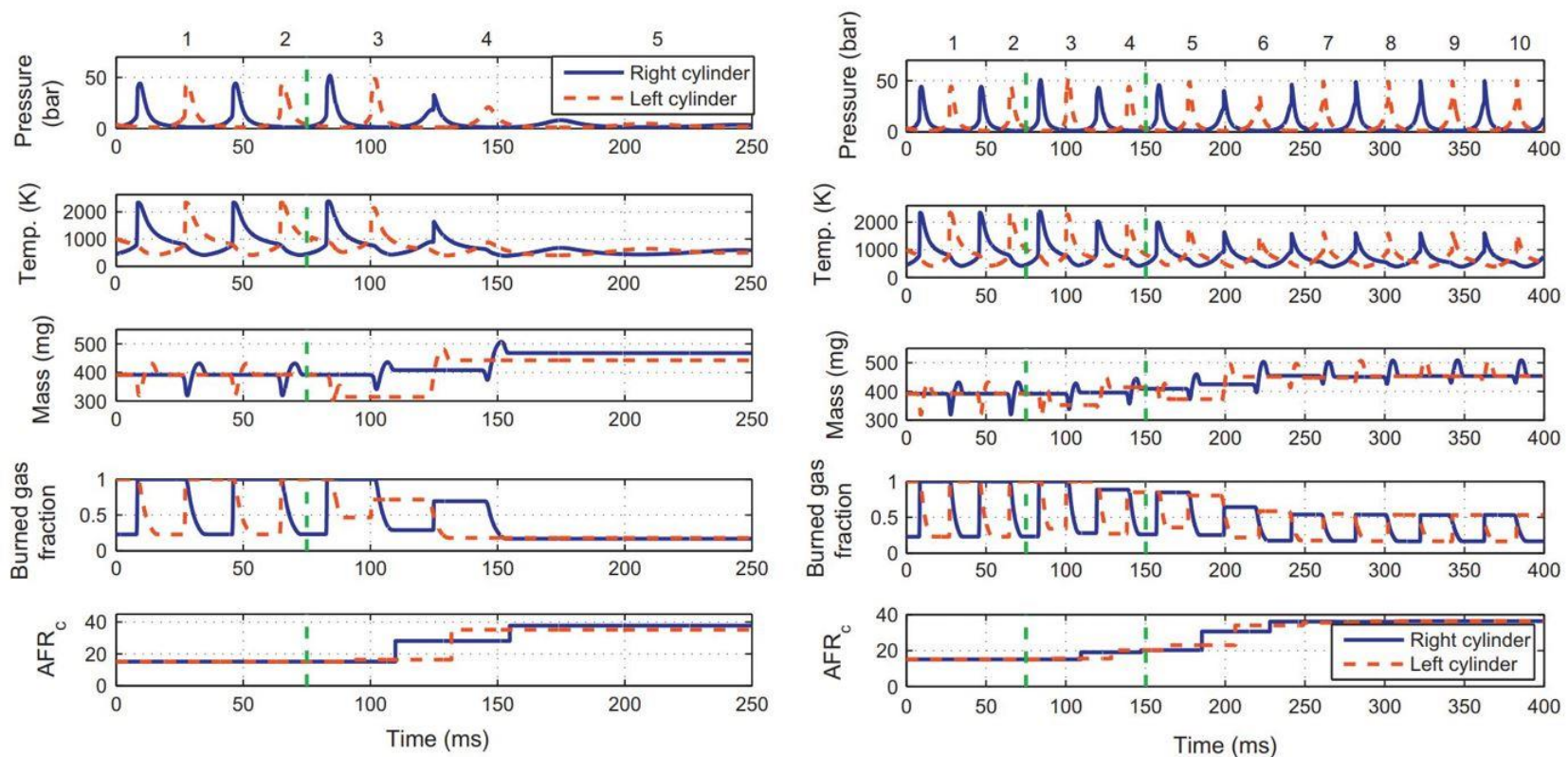

High power SI $\rightarrow$ HCCI mode

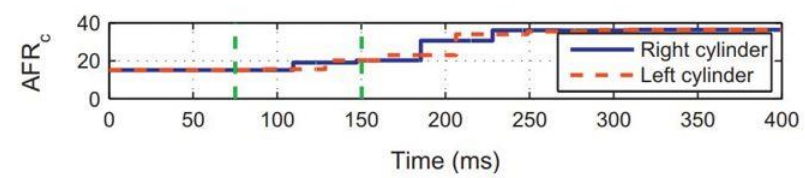

High power SI $\rightarrow$ lean medium power SI $\rightarrow$ HCCI mode

Figure 8: Charge conditions during transition from SI \& lean $\mathrm{SI}$ to $\mathrm{HCCl}$ mode operation in an FPEG system [62]. 
As shown in Figure 8, the FPEG operated successfully, when switched from the lean SI combustion at a medium power level to $\mathrm{HCCl}$ combustion. Initially, at around $75 \mathrm{~ms}$, the LEM electromagnetic force was reduced. This resulted in a higher in-cylinder pressure and compression ratio in the next cycle. Finally, at $150 \mathrm{~ms}$, the spark ignition was switched off and the fuel level and valve closings were adjusted to suit the $\mathrm{HCCl}$ combustion. In the initial phases of transition, a minor trapped mass imbalance was observed in cycle 5. However, the trapped mass imbalance was less severe in this case when compared to the first case. Moreover, the compression ratio was adequate for $\mathrm{HCCl}$ combustion initially and then higher compression ratios were observed in the later cycles i.e. cycle 6. The simulation results also showed that HCCI FPEG achieved lower peak cylinder temperatures and better efficiency than the stoichiometric SI FPEG system.

Kleeman et al. investigated the performance of an HCCI FPEG system with increased levels of EGR [85]. A computational methodology for dynamics and combustion was developed based on the iterative convergence calculations. The results were then validated with the chemical kinetics reaction code SENKIN. The simulation results demonstrated indicated efficiencies above 50\%. Low emission levels of soot and $\mathrm{NO}_{\mathrm{x}}$ were also obtained through a high compression ratio and elevated EGR rates. In addition to that, the influence of injection timing and spray cone angle on combustion characteristics was also studied. As the injection spray angle was reduced, the peak in-cylinder pressure and IMEP rose for the same injection timing. Near zero soot and $\mathrm{NO}_{x}$ emissions were reported at an optimized spray angle of $30^{\circ}$. At a very large and a very small cone angle, the particulate matter emissions increased drastically. This is due to the droplets impinging upon the cylinder liner and improper mixing respectively [85]. Based on the spray angle and injection timing parametric studies, an indicated efficiency of $52 \%$ was obtained for the optimized case.

Hung et al. investigated the influence of intake conditions, engine load, spring stiffness and spark timing on the transition from SI to $\mathrm{HCCl}$ combustion mode [63]. The results presented by Hung et al. [63] matched with the results presented by Chiang et al. [62]. The simulation results reported successful $\mathrm{HCCl}$ transition from SI combustion by regulating intake conditions, ignition timing, springs stiffness, equivalence ratio, and engine load. During the transition, the engine load and the intake temperature were increased as the equivalence ratio was decreased. Moreover, an increase in the intake pressure from 1.1 bar to 1.6 bar reduced the in-cylinder peak temperature in the $\mathrm{HCCl}$ combustion mode. Besides intake pressure, spring stiffness variation at the modeling 
level was also used as an alternative to reduce the peak gas temperature. Finally, the indicated performance in an $\mathrm{SI}$ combustion was found to be lower than the $\mathrm{HCCl}$ combustion. Even though many simulations are reported on $\mathrm{HCCl}$ combustion, the stable experimental prototype is yet to be investigated.

\subsection{FPEG control systems}

In an FPEG system, it is essential to match the engine power output to the generator demand by using an external control system. In an FPEG system, if the LEM consumption goes beyond the engine combustion output, then the stroke length, peak cylinder pressure, velocity at mid-stroke will be reduced for following cycles. As a result, the system will "ring down" to a stall. If the engine combustion delivers too much energy, the stroke length and compression ratio will increase on the following cycle. At mid-stroke, this same combustion energy is vested in high translator velocity, associated with an increase in stroke length. In the end, if more engine power is delivered, losses from in-cylinder heat transfer, increased ring friction and mass loss past the rings will consume the excess power. However, this operating condition is unlikely to be optimal or efficient. This results in the mechanical damage to the system which is similar to the conventional engine operating at too high speed. Moreover, the stable operation and performance of an FPEG depend upon the type of control architecture employed in the system.

Currently, there exist two different kinds of control strategies for the FPEG operation. The first control strategy refers to motion or trajectory-based control (the piston motion is controlled to follow the specified reference motion). The second control strategy allows the LEM to follow a specified current profile for stable operation. Both strategies employ system energy balance approach for the stable FPEG operation. Apart from them, a control strategy is required for starting of the FPEG system. The section below summarizes the control strategies used in the FPEG system for stable operation.

\subsubsection{Starting process control}

During the start-up of an FPEG, the centrally mounted LEM operates as a motor until desired combustion conditions are met. The startup controller uses the sign change in the piston velocity at the dead centers. This reversal control is one of the complex controls in an FPEG system. Sun et al. investigated a reversal control method and energy utilization efficiency during the starting 
process of an FPEG system [91]. A mathematical model was developed by using a position tracking mode and a velocity tracking mode for starting force reversal. The position tracking was achieved by using a linear displacement sensor. The velocity tracking was achieved by estimating the sign change location of the piston velocity. By using these two tracking modes, the motoring efficiency was comparatively analyzed.

The simulation results showed that both position and velocity tracking modes started the FPEG system steadily. For a starting force magnitude of $180 \mathrm{~N}$, the piston reached the ignition favorable conditions in the fourth stroke for the velocity tracking mode. The same conditions were attained in the fifth stroke for the position tracking mode [91]. The main reason for the inefficiency in the position tracking mode was due to piston moving forward during the starting force reversal. Based on the variable reversing positions, an optimized position tracking mode was adopted.

Jia et al. investigated a closed loop and an open loop control strategy for starting the FPEG system [79]. Based on their simulation results, the open loop control was found to be inferior when compared to the closed loop control. Moreover, they considered the starting by using mechanical resonance and one-stroke methods. The one-stroke method is the starting process in which the combustion favorable conditions were attained within one stroke. With the proposed closed loop control, a magnitude of $110 \mathrm{~N}$ and $800 \mathrm{~N}$ of motor force was utilized when started by mechanical resonance and one-stroke methods for the same dynamic conditions. Based on the results, the FPEG startup with mechanical resonance was found to be highly efficient in terms of input motor force $[79,80,81]$.

\subsubsection{Transient piston motion control}

The piston position in an FPEG system is measured by using a linear displacement sensor. For a two-stroke engine, the piston motion control is important for effective scavenging performance. Yang et al. presented a piston motion controller approach for an FPEG system based on a Linear Quadratic Regulator (LQR) technique [92]. They tracked a specified clearance set point at the dead centers for a controller input of fuel mass. Nemecek et al. examined piston motion control of a two-stroke opposed FPEG system by providing a reference trajectory. The reference trajectory provided was very similar to a sinusoidal signal [93]. Their simulation results showed that the deviation in the reference trajectory had a negative impact on FPEG performance. 
Kosaka et al. from Toyota investigated the effect of piston motion on the combustion and power generation characteristics of an FPEG system [94]. Unlike clearance set points, they designed a controller to ensure a stable compression ratio. With the proposed control design, stable FPEG operation was accomplished for more than 4 hours. Moreover, the cycle-to-cycle variations in IMEP were found to be less than $3 \%$. The same control architecture was developed by Robinson et al. for a dual FPEG system with mechanical springs [45]. They also reported a steady FPEG operation with reduced cycle-to-cycle variations (Figure 9).

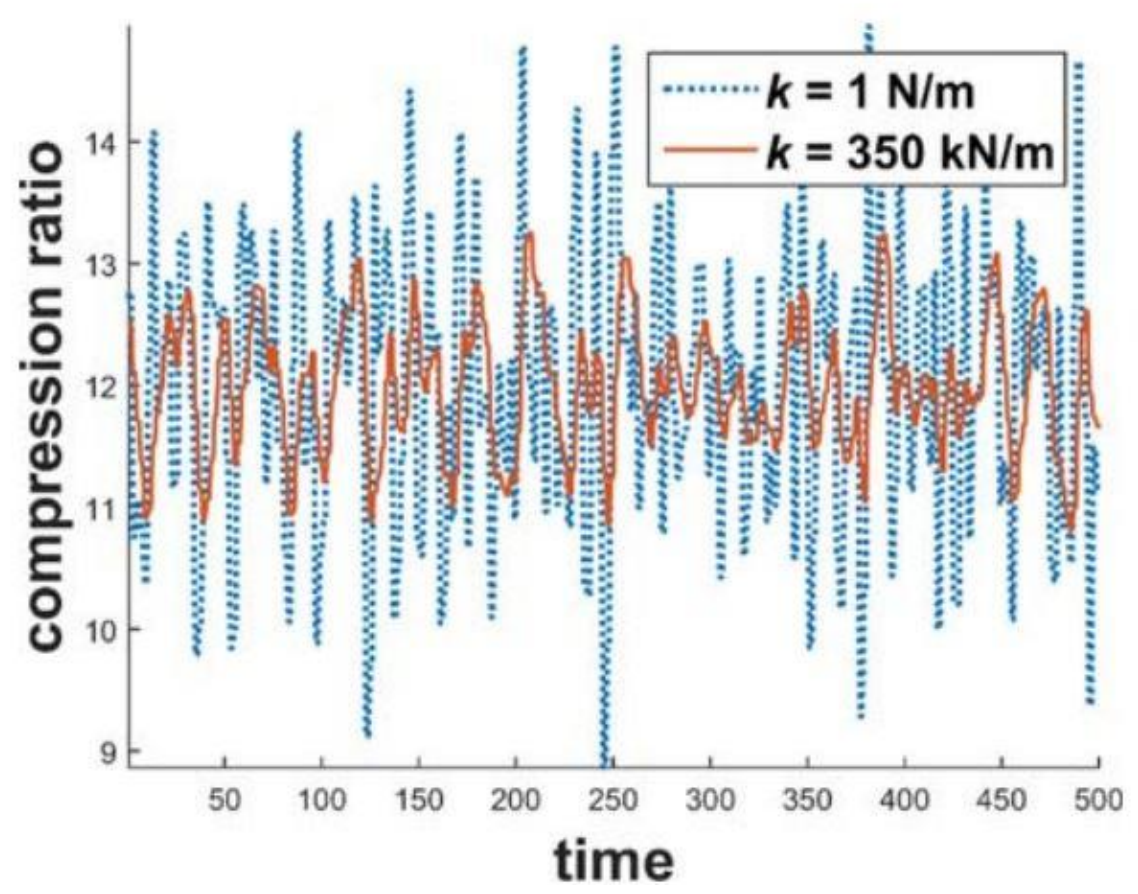

Figure 9: Cycle to cycle variation with trajectory piston motion control approach [45].

Mikalsen and Roskilly investigated the response of an FPEG system with a wide variety of controllers [95]. The controllers included a robust Proportional-Integral-Derivative (PID) controller, a Pseudo-Derivate Feedback (PDF) controller and a disturbance feedforward controller. At moderate loads, the PDF controller with disturbance feedforward was found to be more suitable than that of a conventional PID control. This was due to the better load handling capabilities of the PDF controller. They also stressed the importance of a robust controller architecture for an FPEG system with rapid cycle-to-cycle variations. 
Zhang et al. examined a trajectory-based combustion control in an FPEG system [96]. Simulations were performed for an FPEG operation with seven renewable fuels, including ethanol, syngas, dimethyl ether, biodiesel biogas, hydrogen and Fischer-Tropsch fuel. The Fischer-Tropsch fuel is a liquid hydrocarbon fuel obtained from the chemical reactions of carbon monoxide and hydrogen. The effects of both piston motion and compression ratio on the combustion process were considered [97]. The simulation results showed an improvement in the thermal efficiency by $5 \%$ by changing the piston motion patterns when operated on dimethyl ether. Further, asymmetric piston trajectories were also analyzed to enhance the engine thermal efficiency and to reduce the $\mathrm{NO}_{x}$ emissions simultaneously. For an FPEG system operated on hydrogen, the thermal efficiency was relatively improved by $8 \%$, whereas the $\mathrm{NO}_{x}$ emissions were relatively reduced by $70 \%$ with an optimized piston trajectory.

\subsubsection{LEM control}

The FPEG can be controlled either by using an engine control or a LEM control. While the engine dynamics controls are presented in the previous section, this section summarizes various LEM control strategies. Xu et al. employed a constant electromagnetic force profile control strategy based on the reference current profile obtained during one stroke [98]. This reference current being the control variable was used to limit the deviations of dead center positions within a certain range. Xia et al. developed a hierarchical hybrid control strategy composed of a supervisory and a continuous controller [99]. The supervisory control issued commands for valve operation, fuel injection and continuous controller. The continuous controller computed the LEM electromagnetic force based on continuous measurement. The simulation results reported a steady and satisfactory FPEG performance.

Jia et al. employed an overall system control structure with a multi-layer approach for an FPEG system [100]. They stated that the proposed multi-level control strategy was feasible to attain a stable FPEG performance within $0.5 \mathrm{~s}$ for both immediate increase and decrease in load. Bade et al. employed a control strategy for controlling the LEM electromagnetic force with mechanical springs in the final design [51]. The electromagnetic force was modeled as a function of velocity and controller gain. This controller gain scaled the LEM force according to the variation in the compression ratio. Zhang et al. examined a ladder-like electromagnetic force strategy for improving the electrical output of the FPEG system [97]. The electromagnetic force profile was divided into four ladder stages during one operating cycle. Further, a comparative analysis was 
performed for multiple control algorithms as shown in Figure 10. These control algorithms include a strategy with a constant electromagnetic force (strategy 1 ), a strategy with an electromagnetic force proportional to velocity (strategy 2 ) and a ladder-like control strategy based on a reference current profile (strategy 3). Of all the three control strategies, the strategy 3 achieved the highest power output. This power output was relatively $10 \%$ higher than the other strategies.

(a) Strategy 1 and Strategy 3

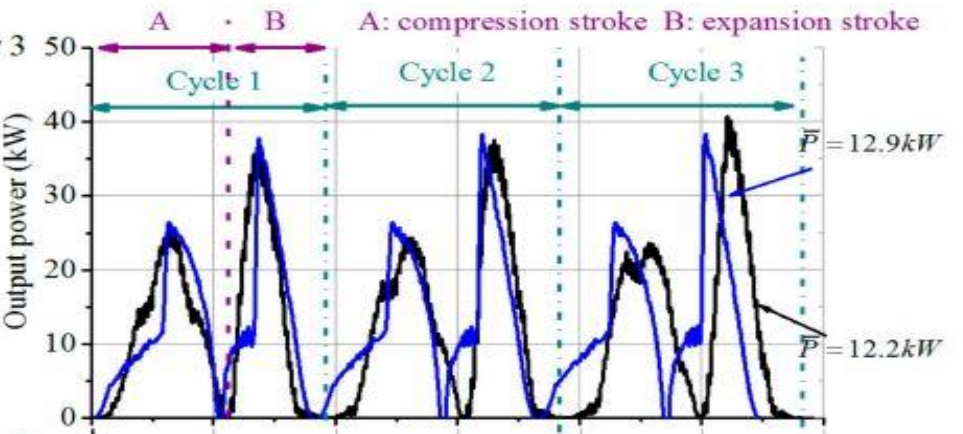

(b) Strategy 2 and Strategy 3

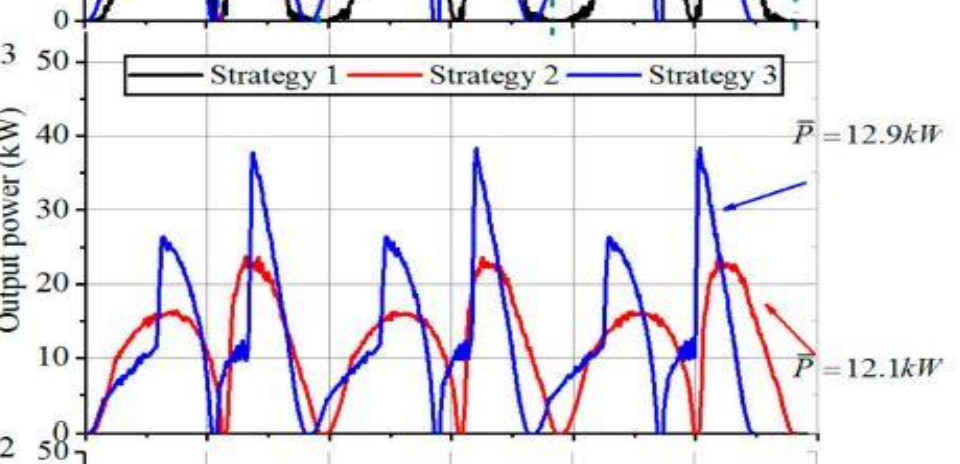

(c) Strategy 1 and Strategy 2

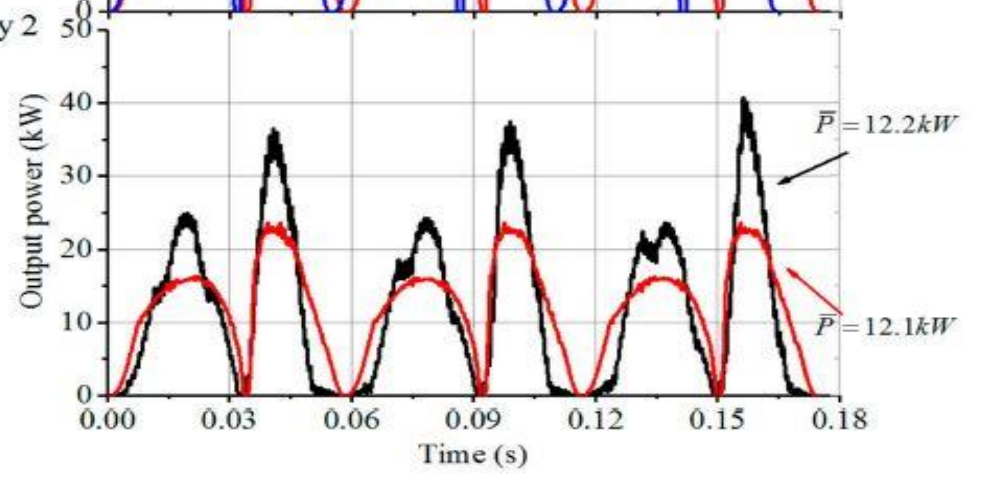

Figure 10: Instantaneous output power comparison for a single cylinder, spring assisted FPEG with multiple LEM control strategies [97].

\subsection{FPEG gas exchange process}

The unique FPEG piston motion exhibits variations in the gas exchange process. In a two-stroke FPEG, the gas exchange process is mainly achieved by using exhaust and scavenging ports. 
Based on the gas flow direction, the scavenging process is further classified into cross scavenging, loop scavenging, uniflow scavenging, and hybrid loop scavenging. In a four-stroke FPEG, the gas exchange procedure is achieved by using intake and exhaust values mounted on the cylinder head. The upcoming section presents the gas exchange studies employed in twostroke and four-stroke FPEG systems.

\subsubsection{Two-stroke FPEG gas exchange}

Most of the FPEG prototypes reported in the literature employ a two-stroke thermodynamic cycle owing to its simplicity in operation [26, 51, 46]. Goldsborough and Van Blarigan investigated the scavenging process of a two-stroke FPEG system at the modeling level [32]. A wide variety of scavenging options including loop, hybrid loop, and uniflow scavenging was analyzed. The loop scavenging employed one exhaust port and two symmetric and opposing intake ports. The hybrid loop design enabled the opening of intake ports after the closure of exhaust ports. In a uniflow scavenging configuration, the exhaust gas and fresh mixture moved in the same direction. Based on modeling studies, the hybrid loop and loop scavenging configurations were found to be inferior to the FPEG system. In terms of scavenging efficiency, the uniflow scavenging achieved the highest scavenging efficiency of $85 \%$.

With the uniflow scavenging geometry, four different schemes were further investigated [32]. The four cases include standard uniflow scavenging (case 1), low pressure uniflow scavenging (case 2), stratified scavenging (case 3), and over-expanded cycle (case 4). Of all the four cases, the stratified scavenging process with low intake temperature and intake pressure optimized the efficiency and emissions. The increased thermal efficiency in case 3 was due to an increase in scavenging efficiency. This increase in the scavenging efficiency increased the indicated power. Moreover, they also stated that without proper control, the cycle-to-cycle variations led to increased short-circuiting losses. The results from various cases are presented in Table 1. 
Table 1: Operating results for designs of uniflow scavenging by SNL research team [32].

\begin{tabular}{|c|c|c|c|c|}
\hline Parameter & Case 1 & Case 2 & Case 3 & Case 4 \\
\hline$\eta_{s c}$ & $83 \%$ & $83 \%$ & $93 \%$ & $84 \%$ \\
\hline$\eta_{t r}$ & $99.70 \%$ & $99.50 \%$ & $98.80 \%$ & $99.40 \%$ \\
\hline$m_{\text {del }}$ & $0.96 \mathrm{~g}$ & $1.15 \mathrm{~g}$ & $1.03 \mathrm{~g}$ & $0.92 \mathrm{~g}$ \\
\hline$W_{\text {cycle }}$ & $480 \mathrm{~J}$ & $790 \mathrm{~J}$ & $644 \mathrm{~J}$ & $697 \mathrm{~J}$ \\
\hline$W_{\text {comp }}$ & $24.6 \mathrm{~J}$ & $29.7 \mathrm{~J}$ & $45.1 \mathrm{~J}$ & $35.6 \mathrm{~J}$ \\
\hline$W_{\text {fric }}$ & $19.2 \mathrm{~J}$ & $31.2 \mathrm{~J}$ & $24.3 \mathrm{~J}$ & $59.9 \mathrm{~J}$ \\
\hline$\eta_{\text {th }}$ & $47.50 \%$ & $47.50 \%$ & $52.50 \%$ & $49.10 \%$ \\
\hline$C_{3} H_{8}$ & $67 \mathrm{ppm}$ & $133 \mathrm{ppm}$ & $206 \mathrm{ppm}$ & $136 \mathrm{ppm}$ \\
\hline $\mathrm{NO}$ & $281 \mathrm{ppm}$ & $707 \mathrm{ppm}$ & $94 \mathrm{ppm}$ & $560 \mathrm{ppm}$ \\
\hline Power & $18.7 \mathrm{~kW}$ & $23.1 \mathrm{~kW}$ & $24.6 \mathrm{~kW}$ & $25.7 \mathrm{~kW}$ \\
\hline
\end{tabular}

Mao et al. examined the scavenging and trapping efficiencies of an FPEG system for best parameter combinations of effective stroke length, port overlapping, intake pressure and operating frequency [101]. The computational results showed that the parameters leading to high scavenging efficiencies caused low trapping efficiencies. Based on the simulation findings, a scavenging arrangement of low bore to effective stroke ratio, low intake pressure, and long overlapping distance achieved optimum scavenging and trapping efficiencies of $90 \%$ and $80 \%$ respectively. Sofianopolous et al. investigated the gas exchange process in a loop scavenged $\mathrm{HCCl}$ FPEG system [102]. The CFD study analyzed the effects port target point, inclination angle, port location and airbox compression ratio on the scavenging and trapping performance. The CFD results showed that a high inclination angle of scavenging ports along with a moderate airbox compression ratio achieved optimal scavenging and trapping efficiencies.

Wu et al. also investigated the influence of intake port height, intake port angle and operating frequency on the scavenging performance of a hydraulic FPEG [36]. Based on the parametric studies, a relative scavenging efficiency improvement of $11 \%$ was observed for an optimized design. Carter and Wechner of Pempek Systems Pty. Ltd reported a novel gas exchange design [23]. The novel design with a compressor and a passive intake valve in the piston head is shown in Figure 11. The seated intake poppet valve operates passively without any external controls. It is governed by the pressure of the combustion chamber and compressor, dynamics of mover and gas spring force. During generating mode, this passive intake valve operation is synchronized to the exhaust valve operation. The synchronization is obtained by an electronically controlled high- 
speed actuator. With this novel design, the uniflow scavenging process is expected to be completed in the fastest time possible than the conventional design. Moreover, it also allows the combustion gases to expand for the longest time possible.

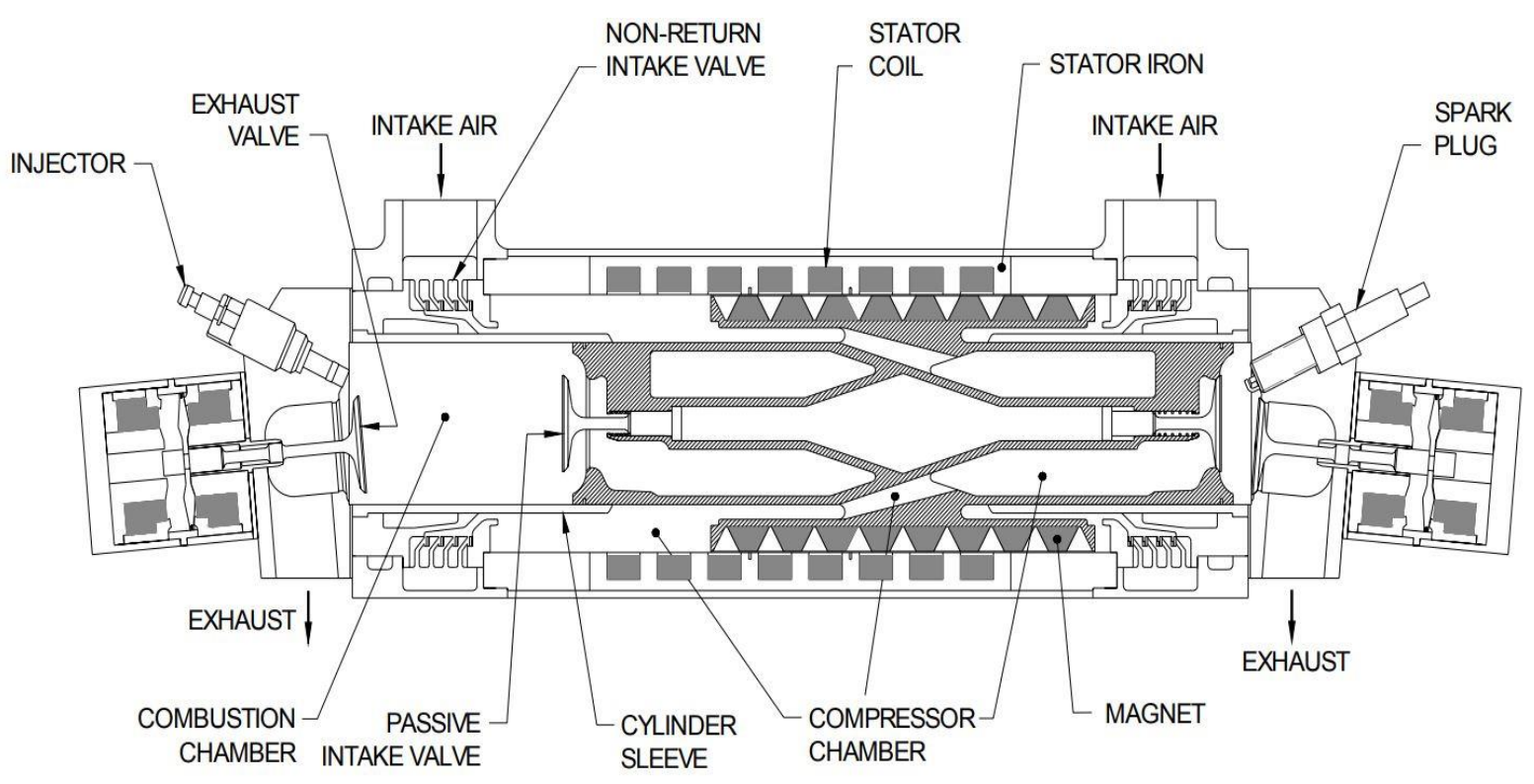

Figure 11: Cross section of a single free piston engine developed by Pempek systems [23].

\subsubsection{Four-stroke FPEG gas exchange}

Due to the complexities of valve control, only a few gas exchange investigations using valves are reported in the literature. Babajimopolous et al. investigated the effects of valve events on the gas exchange process of an HCCI FPEG system by using a CFD model [103]. The CFD simulation results were then compared with the experimental data. The results showed that a late intake valve opening accompanied by an early exhaust valve closing has the potential for effective $\mathrm{HCCl}$ combustion control. Further, a variable valve actuation strategy helped in modulating the internal EGR and engine load. With this strategy, the FPEG system was operated on a wide range of internal EGR ranging from $6 \%$ to $66 \%$.

\subsection{Successful FPEG prototypes}

The increased attention of HEV technology and efficient energy conversion are responsible for increased contemporary FPEG investigations. Though the FPEG has a wide range of 
applications, this section only focuses on the electricity generation prototypes. Moreover, the experimental prototypes in this dissertation are mentioned according to the research group.

\subsubsection{West Virginia University research group}

Researchers from WVU were the first academic researchers who reported the successful operation of an FPEG. Two research papers involving a fundamental analysis and experimental verification were published by Clark et al. in 1998 [34, 43]. The first paper described the FPEG dynamics modeling using Newton's second law. The combustion was considered by using an Otto cycle [44]. The final second order differential equation was solved to provide insights into the system. The second publication described a prototype model built from off-the-shelf components. A two-stroke FPEG prototype with a maximum stroke of $50 \mathrm{~mm}$ and a cylinder bore of $36.5 \mathrm{~mm}$ was operated on an SI combustion cycle. The system was designed for a maximum compression ratio of $8: 1$ and operated at a frequency of $23.1 \mathrm{~Hz}$. An electrical power output of $316 \mathrm{~W}$ was developed [34]. More recently, researchers at WVU aim to develop a $1 \mathrm{~kW}$ FPEG prototype with mechanical springs in the final design [2]. The use of stiff springs allows the system to operate at the frequencies in the range of $65 \mathrm{~Hz}$ to $90 \mathrm{~Hz}$.

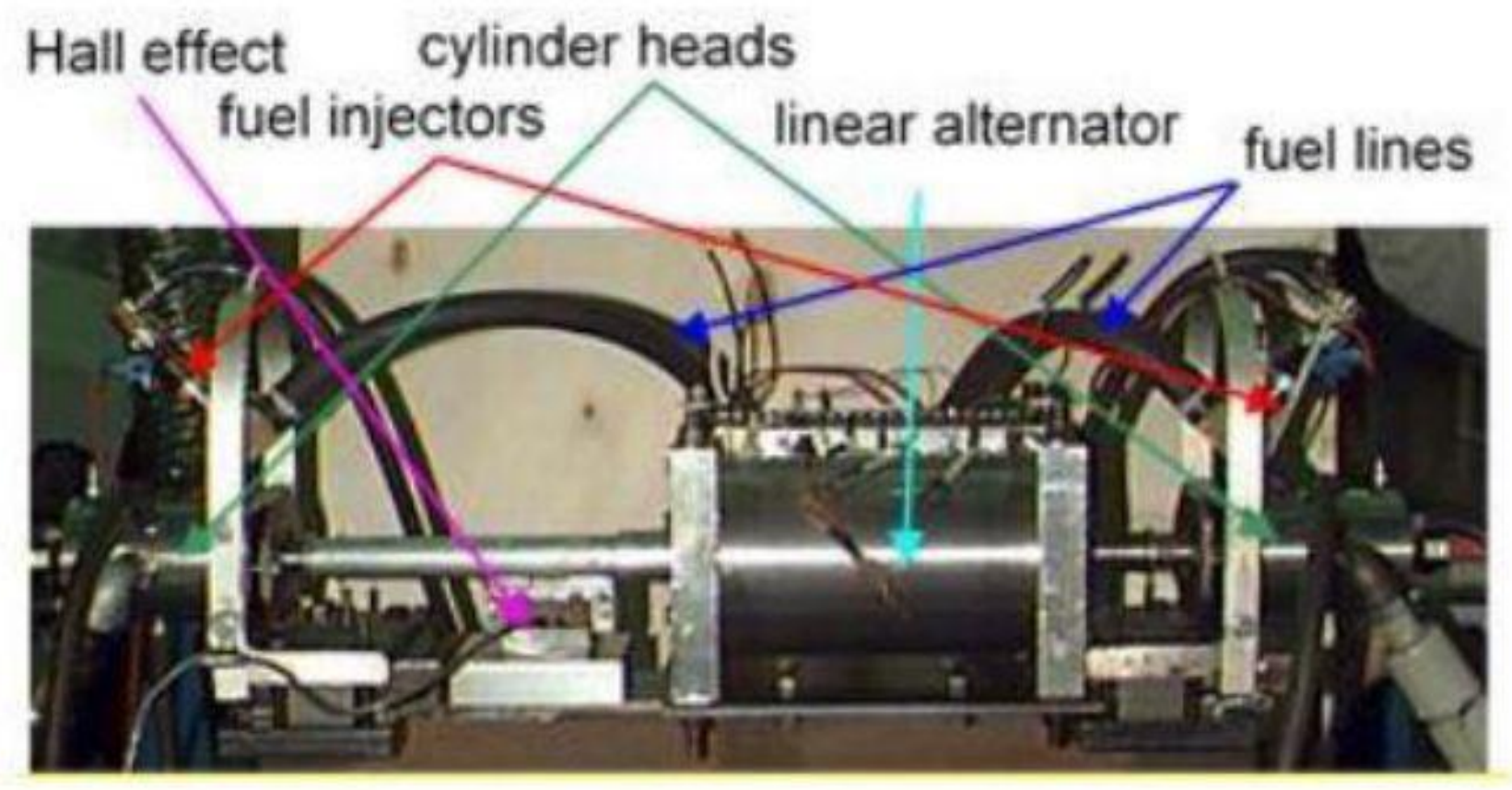

Figure 12: Successful FPEG prototype from WVU in 1998 [34]. 


\subsubsection{SNL research group}

Researchers from SNL started testing their opposed piston FPEG prototype since 2012. The main objective of their prototype is to use it as a range extender for HEV's. Successful demonstration of a hydrogen fueled HCCI FPEG prototype was reported [37]. Low fuel to air equivalence ratio $(\phi)$ mixtures of 0.30 to 0.35 were ignited by $\mathrm{HCCl}$ with compression ratios varying from $20: 1$ to $70: 1$. For a cylinder bore of $81.2 \mathrm{~mm}$, a nominal stroke of $220 \mathrm{~mm}$ and an operating frequency of $34 \mathrm{~Hz}$, the indicated and overall efficiencies of $50 \%$ and $33.6 \%$ were achieved in most cases. Considering the LEM and mechanical efficiency, an electrical power output range of 16 to $19 \mathrm{~kW}$ was reported [37]. Moreover, they also reported that the engine was not steady enough to generate reliable engine out emissions data.

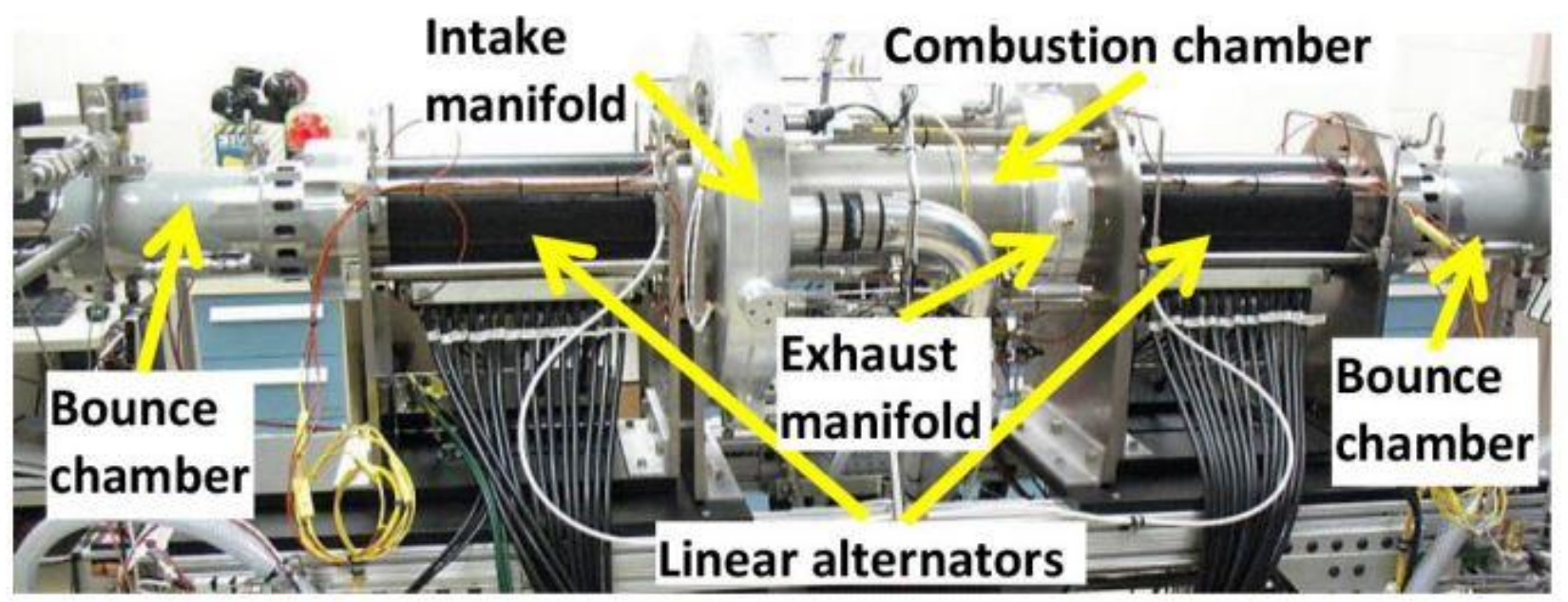

Figure 13: FPEG prototype from Sandia Research Group [37].

\subsubsection{Toyota research group}

In 2015, the researchers from Toyota published an article involving the development of a singlecylinder, $10 \mathrm{~kW}$, gas spring assisted FPEG as an auxiliary power source in HEV's. Kosaka et al. stated the operation of an FPEG prototype with $42 \%$ thermal efficiency and $10 \mathrm{~kW}$ power output through numerical simulations [21]. Experimentally, they reported successful and stable operation of the engine prototype (a cylinder bore of $68 \mathrm{~mm}$, an actual stroke of $90 \mathrm{~mm}$ and an operating frequency of $23 \mathrm{~Hz}$ ) for more than four hours in the limited operational range. Moreover, the cycleto-cycle variations in the IMEP were found to be less than $3 \%$ continuously during that four-hour 
operating window. The results from their simulations were used further in understanding the combustion and generating characteristics of their experimental prototype.

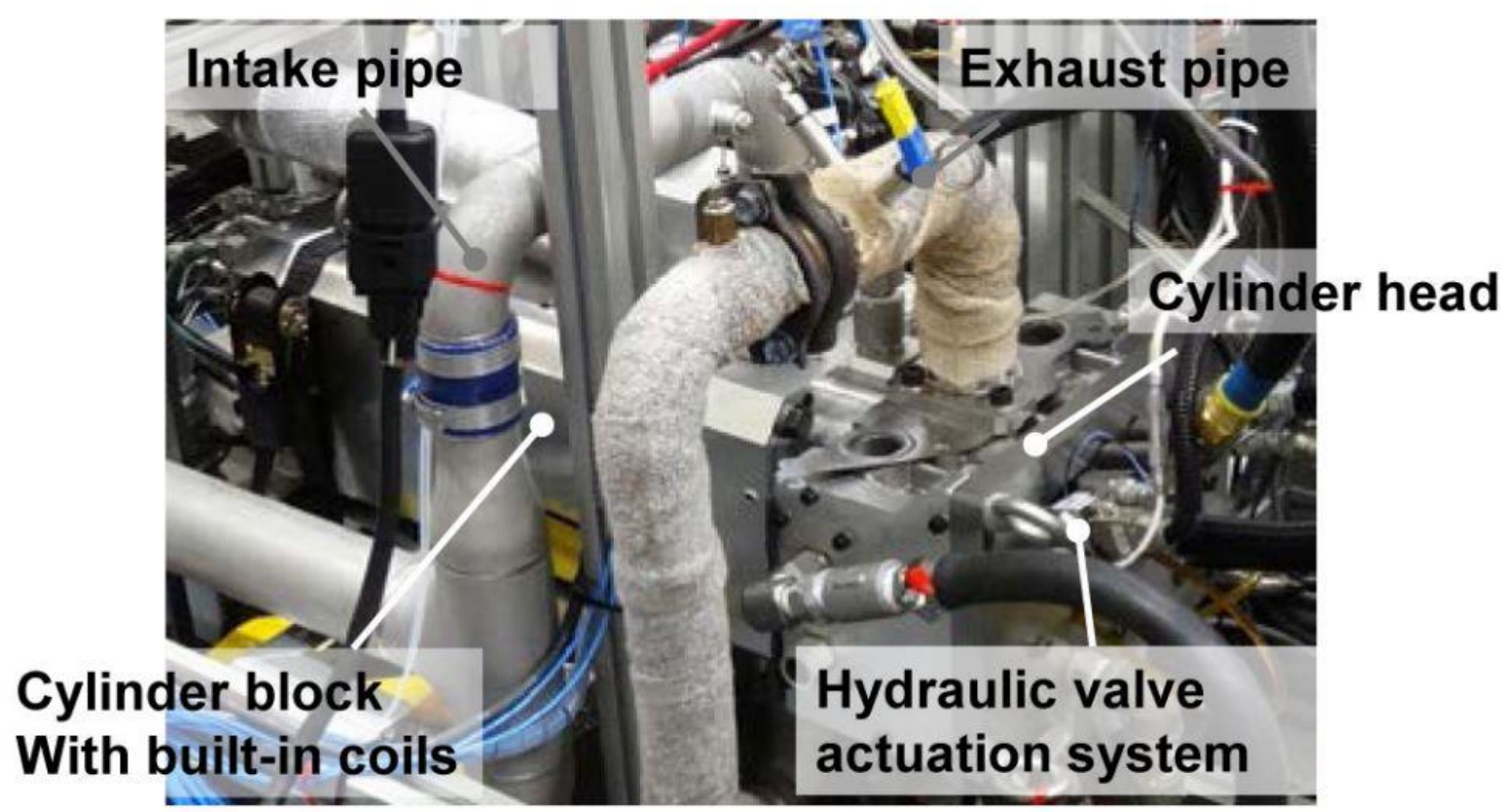

Figure 14: Toyota experimental FPEG prototype [21].

\subsubsection{German Aerospace Center research group}

The researchers at the German Aerospace Center are currently investigating a single-cylinder, direct injection, two-stroke, gas spring assisted FPEG system. The main purpose of their prototype is to use it as an auxiliary power source as well as a CHP system. The FPEG system with a cylinder bore of $82.5 \mathrm{~mm}$ and stroke ranging from 60 to $90 \mathrm{~mm}$ was operated within a frequency range of 16 to $21 \mathrm{~Hz}$ [41]. The experimental prototype showed a maximum IMEP of 6.7 bar and an indicated efficiency of up to $38.1 \%$. The FPEG indicated and electrical power outputs were reported as $5.2 \mathrm{~kW}$ and $2.0 \mathrm{~kW}$ respectively. The indicated to electrical power conversion losses were majorly attributed to the linear bearing frictional losses [41]. 


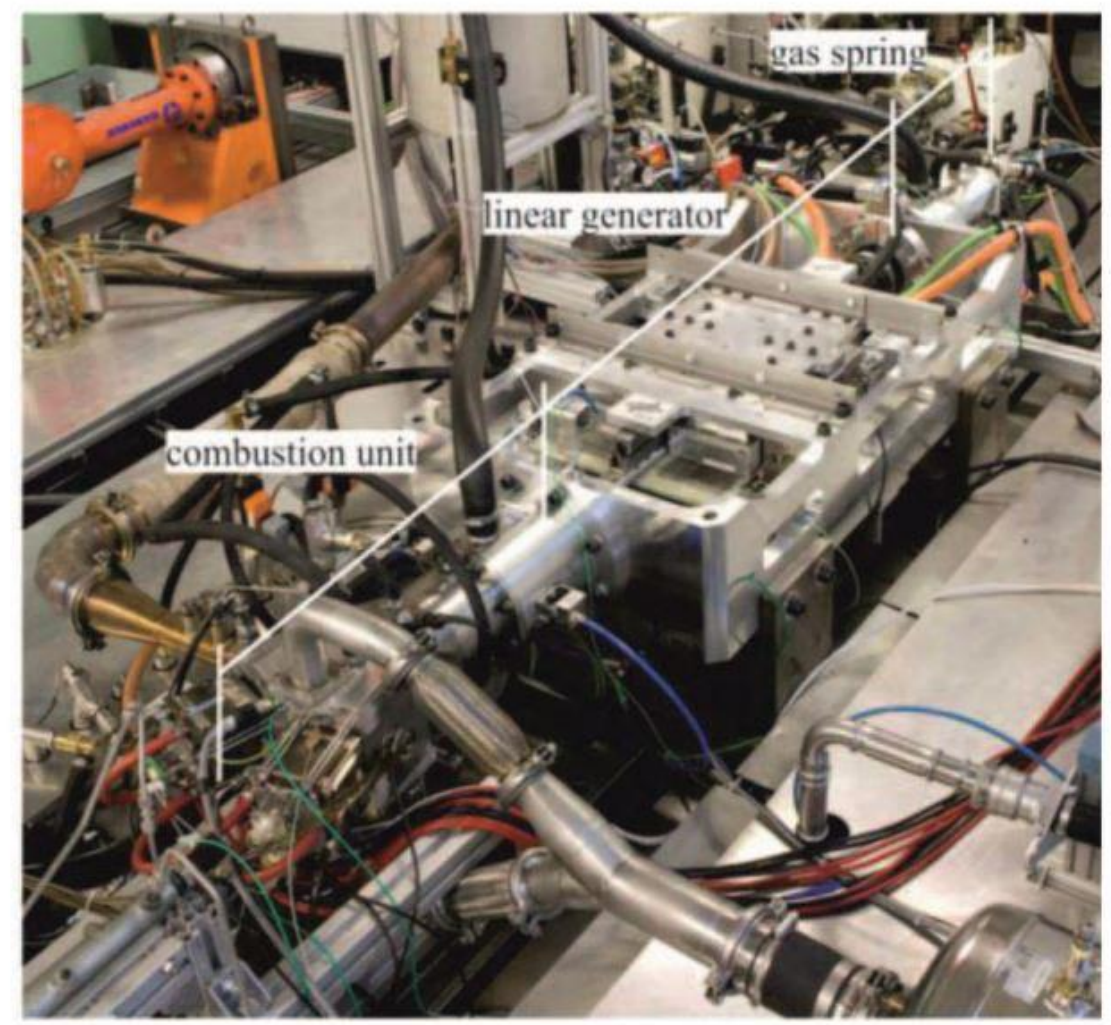

Figure 15: Experimental FPEG system from DLR [41].

\subsection{Research objectives \& methodology}

The main objective of this dissertation research is to model spring-assisted FPEG dynamics and performance, and to apply the modeling results to the development of a $1 \mathrm{~kW}$, spark ignited, natural gas fueled experimental prototype. Further, research emphasis is placed on FPEG with springs that are sufficiently stiff, so that substantial energy is stored in the springs at the ends of the stroke in a cycle. In satisfying this objective, there was a progressive interaction between improving the efficiency and power density of the experimental system by employing the numerical model results. Moreover, a numerical model improvement is also considered with the data and insights gained from the experimental system.

While the main objective served to advance understanding and modeling accuracy of FPEG systems, the research also supported an objective of the US Department of Energy's (DOE) Advanced Research Projects Agency - Energy (ARPA-E). The ARPA-E's objective was to develop a clean-burning, energy efficient $1 \mathrm{~kW}$ natural gas fueled generator. This focused the 
study on a high frequency FPEG operation, and on SI system with stoichiometric combustion mixtures. Even though the numerical model considered ARPA-E targets, it can be expanded to other designs beyond the scope of ARPA-E targets. In this dissertation, the following tasks were identified to meet the overall objectives.

\subsubsection{Task 1: Numerical modeling in MATLAB®/ Simulink}

The first task required a detailed understanding of dynamics and operating characteristics of FPEG and CSE systems of the same size. To meet this objective, a multi-cycle numerical model was developed in MATLAB®/ Simulink for single and dual cylinder FPEG systems. A previous FPEG model in Robinson's dissertation was used as a foundation for the numerical model developed in this dissertation [3]. Improvements were made to the foundational model by incorporating detailed submodels (e.g. piston ring friction, LEM and spring models). Piston dynamics, engine combustion, cylinder heat transfer, engine thermodynamics, gas exchange, friction, motor, generator, and mechanical spring forces were identified as essential processes for the accurate representation of the FPEG system. The corresponding mathematical equations for modeling these processes were obtained based on the literature available for CSE and FPEG systems. A component level modeling with an interchangeable approach was considered with the intention of incorporating a variety of models for any of the system processes. For example, the mechanical spring model can be exchanged for linear and non-linear spring deflection with linear and non-linear damping coefficients. The developed numerical model was then used in understanding the operating characteristics and energy balance insights of FPEG systems ranging from weak or no spring to spring dominant systems.

\subsubsection{Task 2: Prototype Design, Testing and Model Validation}

The second task was to develop an experimental system to demonstrate principles, confirm model predictions, and provide experimental data and results that could be used to further improve the model. A $1 \mathrm{~kW}$, single-cylinder FPEG system was built at WVU for this task. The experimental prototype was evaluated with fumigated and direct injected fuel delivery systems. At the time of writing this dissertation, the FPEG direct injection operation testing was in its preliminary phase. Therefore, the fumigated experimental findings were used to validate the numerical model. 
Two subtasks were developed to validate the numerical model developed in MATLAB®/ Simulink. The first subtask confirmed model predicted dynamics and cylinder characteristics with the experimental data obtained during the starting mode. In other words, this subtask validated the LEM motor submodel by using experimental resonance starting process results. The second subtask validated the model predicted FPEG dynamics and cylinder characteristics during the stable generating mode. In this subtask, a systematic strategy was devised to validate the numerical model at the component level. At first, the deviations in the model predicted dynamics and experimental FPEG dynamics were identified. Next, the model predicted engine cylinder thermodynamics were validated. To achieve this, a heat release function was developed in the numerical model based on experimental cylinder pressure. Finally, a qualitative comparison of voltage, current and electric power generated in the copper windings was made to confirm the predictions of a LEM generator submodel.

\subsubsection{Task 3: Parametric Study \& Sensitivity analysis}

The third task involved a detailed understanding of the FPEG system design space. To achieve this, various sensitivity analysis and parametric studies were carried out to reveal the importance of several assumptions within the numerical model. From an FPEG design perspective, 25 model inputs were identified ranging from engine design parameters, LEM, spring parameters, and operating conditions. Each of the model inputs was analyzed with regard to their impact on effective stroke length, operating frequency, indicated power, FPEG system efficiency, and losses.

In this dissertation, a MATLAB®/ Simulink based Sensitivity Analysis Toolbox ${ }^{\mathrm{TM}}$ was used to compile the numerical model for parametric and sensitivity studies. A code was developed initially for the sensitivity analysis procedure. Later, it was implemented in the numerical model to address the relevance and strength of input variables in deciding the variations in the system outputs. These parametric and sensitivity analysis studies helped in understanding the effect of major deign variables on FPEG steady state behavior. Finally, a framework was developed for the optimization of the experimental FPEG system based on the conclusions from the sensitivity analysis studies. 


\subsubsection{Task 4: Multi-level controller system development}

The fourth and the final task of this dissertation required the development of a multi-level control strategy for stable FPEG operation. In an FPEG system, each independent variable alters the motion with respect to time and therefore alters the engine and LEM response to other governing variables. Initially, a stability analysis was performed with different LEM generator loads to understand cycle-to-cycle variations in the system. The stability analysis identified the interdependency of LEM and engine variables and highlighted the need and importance of the robust control methodology. Moreover, the stability analysis showed this energy imbalance in the model affected sensitivity analysis.

To reduce cycle-to-cycle variations, a multi-level control strategy was proposed at the modeling level. Individual control systems were developed for LEM mode switching, model-based calibration maps, energy management, ignition, and fuel injection timings. These control systems were integrated logically in the Simulink environment. The proposed multi-level controller was developed in MATLAB® Stateflow and was integrated with the FPEG plant system testing at the modeling level. Moreover, various controller inputs were identified to understand the behavior of an FPEG system with different inputs. A feasibility study of an energy management controller with different controller inputs was considered. Finally, an algorithm based on cylinder pressure was devised for optimized ignition timing for a wide variety of operating conditions. 


\section{NUMERICAL MODELING IN MATLAB® / SIMULINK}

This chapter reported the operation of the spring dominant FPEG systems via numerical simulations in MATLABß/ Simulink. More precisely, a spring dominant FPEG system numerical model was developed individually for both single and dual cylinder designs. The developed numerical model included thermodynamics, LEM, combustion, mechanical spring force, frictional force and gas exchange models. The sensitivities of all these submodels were investigated for various loading and operating conditions. Additionally, the variations in the FPEG system dynamics and combustion characteristics were investigated with varying spring stiffness. Finally, the differences in the modeled piston ring frictional losses were presented for both CSE and FPEG systems.

\subsection{Modeled FPEG system overview}

The FPEG systems considered in this research investigation were single and dual cylinder systems. While both single and dual cylinder designs were considered in the numerical model, only a single cylinder design was under development at WVU. The prototype was a two-stroke, natural gas fueled design with an objective of $1 \mathrm{~kW}$ target electrical power output. Stiff mechanical springs were considered as rebound devices. The single cylinder needed the stiff mechanical springs for its operation. In addition, these springs in the modeling and in the final design enabled high frequency FPEG operation. This high frequency operation increased output power and efficiency. For the same electrical power output, the stiff spring addition allowed for more compact FPEG design when compared to the system without springs. Moreover, the FPEG system specifications (i.e. $1 \mathrm{~kW}$, spark ignition and natural gas fueled) were selected based on the objectives of experimental FPEG development at WVU. This prototype FPEG development project was funded and supported by the DOE ARPA-E to the West Virginia University Research Corporation (WVURC) through the GENSETS program under an award number DE - AR000068.

Of all the configurations, the single-cylinder design was simple in construction and easy to operate. It offered the highest power density when mechanical springs were added to the design. Moreover, the addition of stiff springs reduced the cycle-to-cycle variations in the FPEG system. The schematic diagram of the single-cylinder FPEG prototype is shown in Figure 16. 


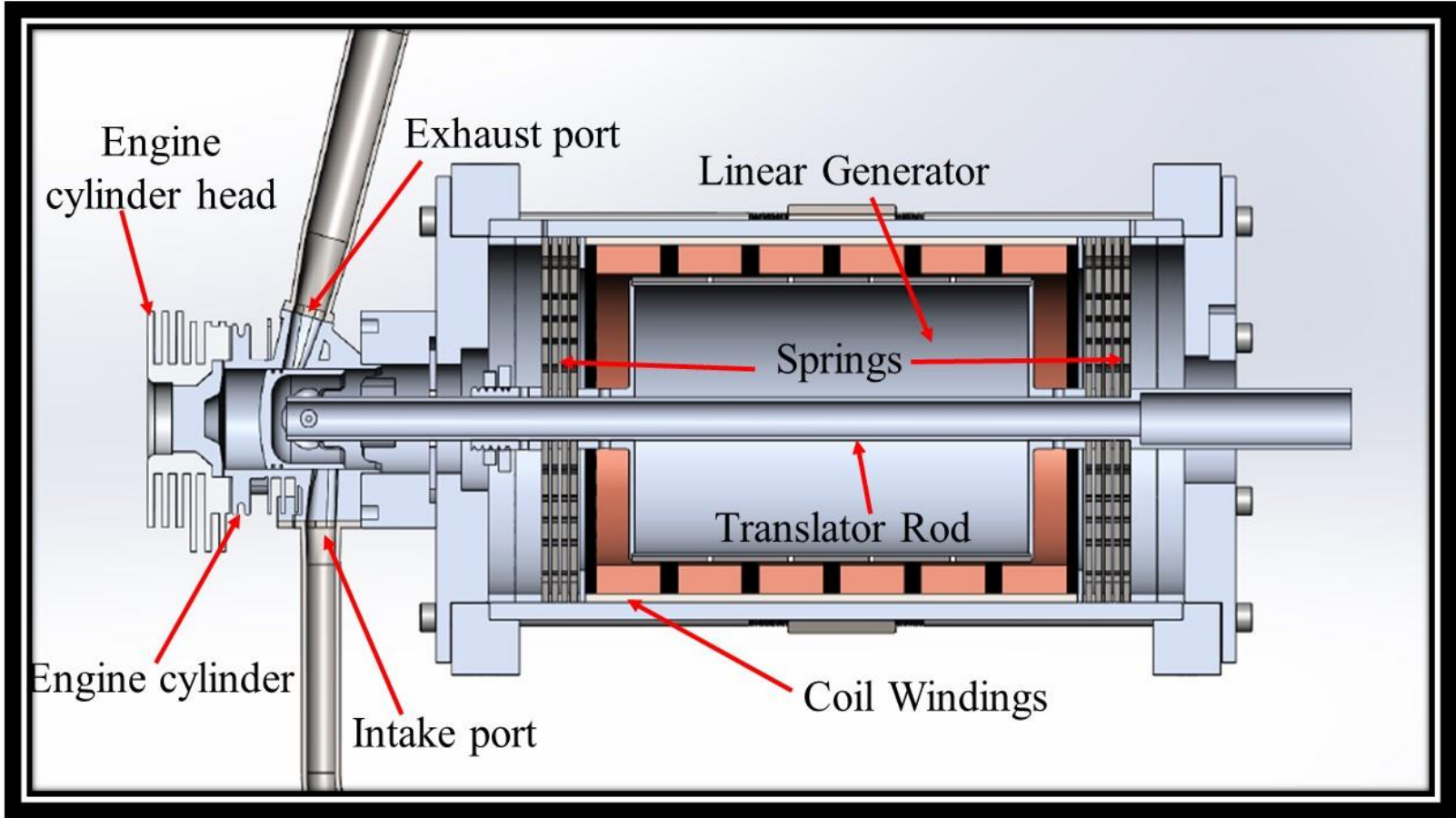

Figure 16: Schematic diagram and experimental prototype of a single cylinder FPEG at WVU.

The essential components of the engine section include a combustion chamber or cylinder on the left, a piston with a single piston ring located internally in the engine cylinder, a crankcase below the intake port, a spark plug mounted in the cylinder head, a resonant intake system, and a resonant exhaust system. Exhaust, intake and scavenging ports were used for the gas exchange process. These ports were at fixed positions. The opening and closing of these ports were defined by the piston displacement. In other words, the scavenging process was coupled directly with the transient piston dynamics.

The LEM section comprised of two main components. A reciprocating tubular drum with permanent magnets attached to it acted as a reciprocating component. The copper windings mounted on the casing acted as a stator. The stiff mechanical springs were mounted on either side of the reciprocating tubular drum. The experimental FPEG system utilized custom-designed flexural springs as shown in Figure 16 [104]. However, at the modeling level, these flexure spring forces were calculated based on spring stiffness and damping coefficients. Before starting the system, these mechanical springs were in their resting position (i.e. neither compressed nor expanded). A translator rod was used to connect the engine piston at one end, springs and the 
reciprocating tubular drum in the central section. The piston assembly (including piston, piston ring, and wrist pin), translator rod, mechanical springs, and a reciprocating tubular drum were the only noteworthy reciprocating components of the current FPEG system. At the modeled level, these reciprocating components were considered as a single mass system with a specified mass input. This mass was connected to the spring-damper system at the modeling level.

Based on FPEG operation, the overall working process of the system was divided into three stages i.e. starting stage, switching stage, and generating stage. Initially, the FPEG system was started in the starting or motoring mode. In this mode, the centrally mounted LEM was operated as an electric motor. The LEM was operated as a motor based on the principle of electromagnetic induction. During the motoring operation, the axial motoring force was provided to the FPEG system. The method of switching the LEM as a motor and the corresponding submodel is presented in Section 2.2.8. With the proposed starting control, the electric motor provided the necessary electromagnetic motoring force to the springs to achieve mechanical resonance. Mechanical resonance is a process in which constant electromagnetic force is provided in the direction of piston velocity until desired combustion conditions are met. The electric motor was managed to provide the electromagnetic force on successive strokes. As a result, the energy available in the system was increased steadily. Finally, the in-cylinder pressure, piston effective stroke length, and trapped compression ratio were increased from cycle-to-cycle by means of the mechanical resonance behavior. During the motoring operation, the control algorithm checked for the combustion favorable conditions in the ICE. The combustion favorable conditions were defined by means of a target trapped compression ratio.

Once the ICE reached the combustion favorable conditions, the fuel was introduced into the engine cylinder. Later, the combustion was initiated by using an ignition system. Due to combustion forces, the piston coupled to the translator rod moved towards the right. Moreover, the combustion forces also caused the mechanical springs to compress during the expansion or power stroke of the engine. Once the piston reached BDC, the mechanical springs expansion forces caused the piston to move towards TDC. This movement caused the compression of fuel and air mixture in the engine cylinder. In other words, the mechanical springs were responsible for the return of the piston during the engine compression stroke. As the piston reached TDC, the fuel and air mixture were combusted again. Later, the entire process was repeated. The period in between the starting mode and the generator mode (i.e. introducing fuel and the initial cycles of combustion) was termed as a switching or transient stage. 
Finally, the combustion forces from the engine cylinder drove the piston, translator rod, and permanent magnets. The translating piston motion created the distortion in the magnetic field generated by the permanent magnets. This magnet field distortion further produced an electric current from the stator copper windings through continuous operation. In other words, an electric power was produced through the copper windings. This stage in the FPEG system operation was termed as the generating stage.

\subsection{Numerical model description}

The numerical model presented in this chapter stemmed from the fundamental and numerical analysis developed by Robinson for a dual cylinder version [3]. The developed numerical model included thermodynamics, LEM operation, combustion, mechanical spring force, frictional force and gas exchange models. All these submodels were connected logically in Simulink to obtain an overall FPEG plant system. In the current numerical model, the plant and control functional blocks representing an actual FPEG and control systems were developed separately. These blocks were interconnected logically in Simulink. The communications between multiple submodels were carried out by using MATLAB® embedded functions. These functions were available within the Simulink blocks. Each Simulink block received numerous inputs and calculated multiple outputs by using mathematical equations.

A time-based simulation approach was considered for all the operations in the numerical model. For example, the numerical model initially calculated the value of the particular variable at a given time step using a differential equation. The value of that variable was calculated in the next time step by using the rate of differentiation and initial value. A fixed time step approach with ordinary differential equation solver of multiple orders was considered. Variable time step solvers failed to capture the mass flowing into and out of the cylinder domain reliably. As a result, a fixed time step solver was considered in this research investigation. A time step of $5 \mu$ s was selected for all the modeling results presented in this document. When the time step was increased above $25 \mu$ s, the numerical model resulted in an unstable operation and eventually stalled the system. The selection of the fixed time step was entirely dependent upon the system operating frequency. Since the current FPEG system was operated at a frequency greater than $75 \mathrm{~Hz}$, a time step of $5 \mu$ s was considered. Even though the numerical model considered ARPA-E targets for validation, the numerical model can be expanded to broader FPEG designs beyond the scope of ARPA-E 
targets. The corresponding mathematical equations used in the development of the numerical model are presented in the sections below.

\subsubsection{FPEG piston dynamics}

In any FPEG configuration, the piston dynamics were defined by using the summation of forces acting on the translator rod. These forces were solved by Newton's second law of motion. The translator rod acceleration was dependent upon the net forces acting on it and the translator mass. According to Newton's second law of motion, the product of mass and acceleration of the translator rod was proportional to the net forces acting on the translator rod. For a single and dual cylinder system, various forces acting on the translator rod are shown in Figure 17.
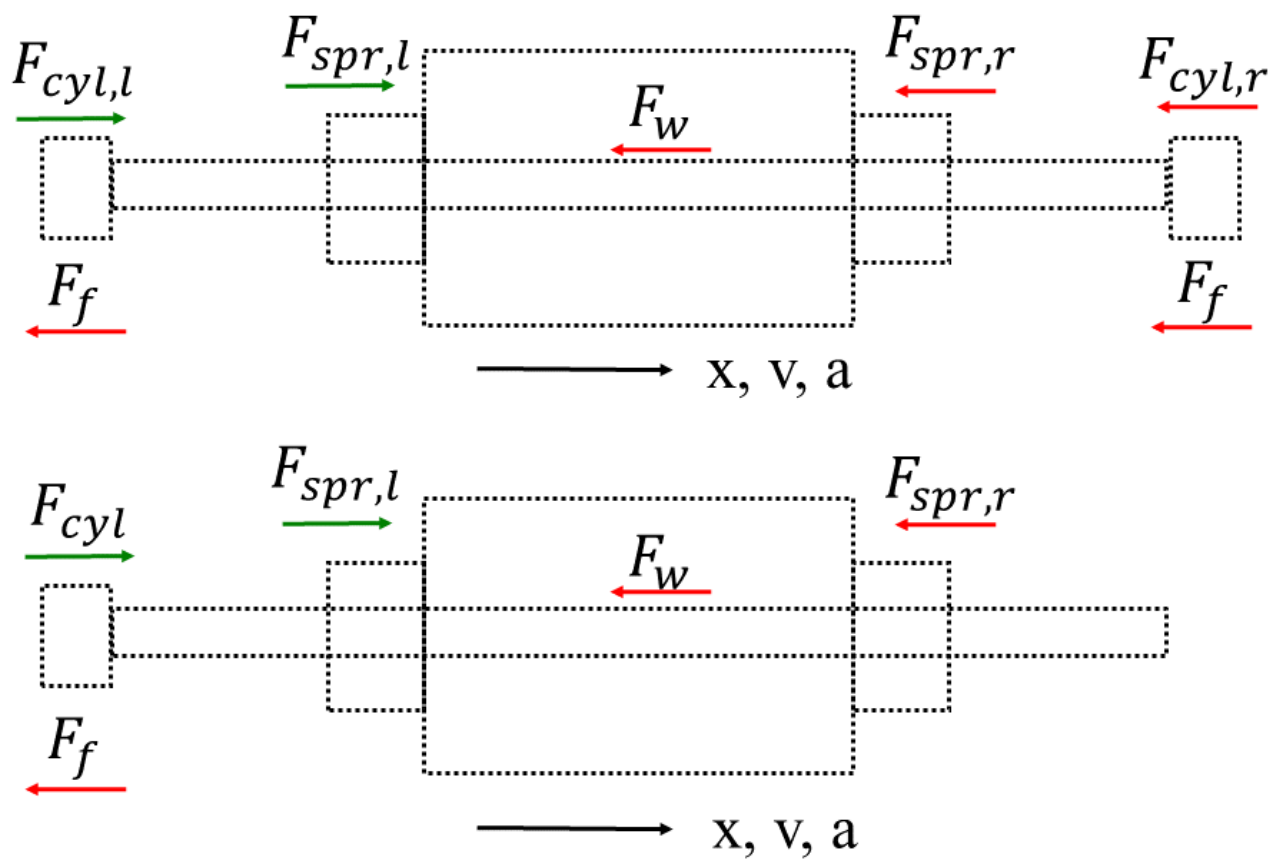

Figure 17: Free body diagram of dual and single cylinder FPEG systems.

In Figure 17, during the piston motion from left to right, the spring forces were positive. Thus, they were represented by a green arrow. On the return stroke, the springs opposed the motion. As a result, they were represented by a red arrow. Moreover, the springs achieved the peak force magnitude at the dead centers. This was due to the maximum spring deflection (i.e. expansion or compression). At the neutral point or mid-stroke location, there was no compression or expansion 
in the springs. As a result, the spring force was zero at the neutral position. Similarly, during the motion from left to right, the left and right engine cylinders were in the expansion and compression stroke respectively. Because of this, the combustion forces from the left and right cylinders were positive and negative respectively. Besides these, the LEM and frictional forces were negative. This was due to the energy taken out from the LEM and energy lost in friction. The corresponding force balance equations are presented in Eq. 1.

$$
\begin{gathered}
m \frac{d^{2} x}{d t^{2}}=F_{c y l}+F_{s p r, l}-F_{s p r, r}-F_{w}-F_{f} \\
m \frac{d^{2} x}{d t^{2}}=F_{c y l, l}-F_{c y l, r}+F_{s p r, l}-F_{s p r, r}-F_{w}-F_{f, l}-F_{f, r}
\end{gathered}
$$

The various forces acting on the translator rod in Figure 17 caused the translator rod to reciprocate. The reciprocating motion was defined by using position $(x)$, velocity $(v)$ and acceleration (a) of the translator rod. In Eq. 1, the subscripts 'cyl', 'spr', 'f', 'w', 'l', and 'r' represent an engine cylinder, mechanical springs, friction, LEM work, left and right respectively. The terms ' $m$ ', ' $t$ ', and ' $F$ ' represent the translator moving mass, time and force correspondingly. The translator moving mass included the mass of the piston assembly, appropriate spring inertia, and LEM moving magnet mass. The green and red colored arrows represent the forces that are supporting and opposing the translator rod motion. While the governing force balance equations were different, the calculations of various forces were the same for both single and dual cylinder FPEG systems.

\subsubsection{Thermodynamics}

In this dissertation, the thermodynamic model was defined by using a simplified one-dimensional open system energy balance equation for the cylinder control volume. The effects of potential and kinetic energy were neglected in the energy balance equation. While the current model employed a simplified approach when compared to the chemical kinetics and CFD simulations, it had a high level of sophistication. Moreover, for a wide range of design possibilities, the computational time for multiple simulations was not possible with a multi-zone CFD model. As a result, the current model balanced the simplicity, sophistication, and computational time over the accuracy of CFD 
models. Initially, the cylinder control volume submodel received the details of specific volume, temperature, and species information from the MATLABß workspace. This information was used to calculate all the essential thermodynamic properties, namely gas constants, enthalpy, and specific heats. Moreover, the gas composition inside the engine cylinder was assumed to be homogeneous. In other words, the mixing properties of the gas composition was neglected.

In the thermodynamics submodel, the whole cycle in the engine cylinder in one cycle was divided into four distinct processes. The processes include combustion, expansion, gas exchange, and compression. The CSE empirical correlations were converted into the time domain to represent the FPEG system. Adopting the CSE models introduced some inaccuracies in an FPEG system. However, these models can be used with confidence, especially when the empirical correlations for FPEG are not available. Moreover, the deviations in the FPEG and CSE model outputs were also be attributed to the error because of using conventional correlations. This was especially true for minimal deviations (i.e. the error was not a real difference between these two systems). Moreover, the positional indications (such as before BDC, after TDC) had no real sense in the FPEG systems. This was because of the variable FPEG dead center positions for a given operating speed. With the details of the engine cylinder processes, the one-dimensional differential equation for the cylinder pressure is represented in Eq. 2.

$$
\frac{d P}{d t}=\frac{-P k}{V} \frac{d V}{d t}+\left(\frac{k-1}{V}\right)\left(\frac{d Q_{\text {in }}}{d t}-\frac{d Q_{\text {out }}}{d t}+\frac{d H_{\text {in }}}{d t}-\frac{d H_{\text {out }}}{d t}\right)
$$

The terms ' $P$ ', ' $k$ ' ' $V$ ', ' $Q_{\text {in }}$ ', ' $Q_{\text {out }}$ ', ' $H_{\text {in }}$ ', and ' $H_{\text {out }}$ ' in Eq. 2 represent cylinder pressure, specific heat ratio, in-cylinder volume, heat addition, heat rejection, inlet enthalpy and outlet enthalpy respectively. The outlet enthalpy also considered the enthalpy lost from the mixture leaked past the piston ring, i.e. blow-by. The inlet and outlet enthalpies were determined by mass inflow $\left(\dot{m}_{i n}\right)$ and outflow rates $\left(\dot{m}_{\text {out }}\right)$, respective gas specific heats at constant pressure $\left(c_{p}\right)$, and temperatures $\left(T_{\text {in }}, T_{\text {out }}\right)$. The inlet mixture temperature was provided as an input to the numerical model. The exhaust temperature was dependent upon the combustion process and exhaust port location in the cylinder. The procedure for the calculation of mass inflow and outflow rates are defined in Section 2.2.3. The corresponding mathematical equations for inlet and outlet enthalpies are represented in the following equation. 


$$
\begin{gathered}
\frac{d H_{\text {in }}}{d t}=\dot{m}_{\text {in }} c_{p} T_{\text {in }} \\
\frac{d H_{\text {out }}}{d t}=\dot{m}_{\text {out }} c_{p} T_{\text {out }}
\end{gathered}
$$

Eq. 3

The inlet mixture entering into the engine cylinder was a combination of both fuel and air during a fumigated operation. The fumigation operation refers to the continuous injection of fuel into the intake stream of air. Hence, the inlet enthalpy considered both the enthalpies of fuel and air in a fumigated FPEG system. However, in case of a direct injection operation, only the air flowed through the inlet port. The fuel was delivered directly in the cylinder domain. Similarly, the exhaust mixture consisted of a burned mixture, unburned fuel and air mixtures from blow-by. Hence, the outlet enthalpy considered the enthalpies of burned mixtures and gas leakage past the piston ring. After calculating the cylinder pressures from Eq. 1, an ideal gas equation was used to calculate the cylinder gas temperature as shown in Eq. 4.

$$
T=\frac{P V}{m R}
$$

Eq. 4

The volume $(V)$ in the above equation was calculated by using the translator dynamics submodel. The mass of individual gas species $(m)$ was calculated by using a species conservation (continuity equation) submodel.

\subsubsection{Intake / exhaust gas exchange}

The current FPEG system under development employed ports for mass transfer. In other words, the exhaust, intake, and scavenging ports were the orifices of mass transfer. The FPEG cylinder domain with intake or scavenging and exhaust ports is shown in Figure 18. From Figure 18, it is evident that these ports are just openings on the cylinder liner. These ports were used for the gas exchange between the combustion chamber and other volumes. In this dissertation, an engine cylinder control volume was assumed homogenous with a constant uniform pressure distribution for intake and exhaust system volumes. Moreover, the flow model operated only if there were two or more associated control volumes (e.g. intake volume and cylinder volume) with a pressure difference. The mixture mass flow rates through these ports were modeled as a compressible 
flow through a restriction of the flow [105]. This compressible flow equation was obtained from a one-dimensional isentropic analysis of gas flow with a discharge coefficient. Alternatively, the opening and closing of the intake and exhaust ports were determined by the translator location. Considering the large pressure differences and small area, the intake and exhaust flows were choked during the initial port opening phase. Mainly, the mass flow rates through the ports were linked directly to the upstream pressure and temperature, downstream pressure, port area, and discharge coefficient. With the information of all these parameters, the mass flow rates from the intake and the exhaust ports are estimated by using the following equation.

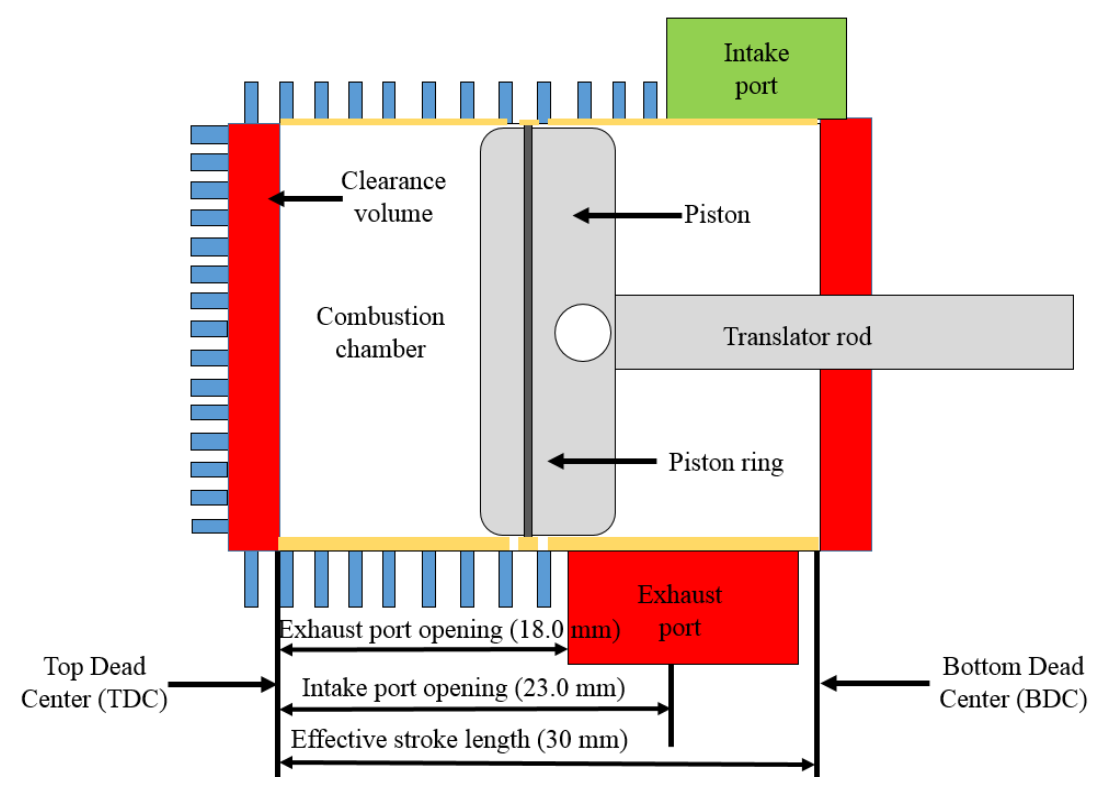

Figure 18: Schematic diagram of an FPEG cylinder domain with intake and exhaust ports.

$$
\dot{m}_{i n}=\frac{C_{d} A_{i n} P_{i n}}{\left(R T_{i n}\right)^{\frac{1}{2}}}\left(\frac{P_{c y l}}{P_{i n}}\right)^{\frac{1}{k}}\left\{\frac{2 k}{k-1}\left[1-\left(\frac{P_{c y l}}{P_{i n}}\right)^{\frac{k-1}{k}}\right]\right\}^{\frac{1}{2}}
$$

If the flow is choked i.e. if $\left(\frac{P_{c y l}}{P_{i n}}\right) \leq\left[\frac{2 k}{k+1}\right]^{\frac{k}{k-1}}$, then the mass flow rate in that case is equal to.

$$
\dot{m}_{\text {in }}=\frac{C_{d} A_{\text {in }} P_{i n}}{\left(R T_{\text {in }}\right)^{\frac{1}{2}}}(k)^{\frac{1}{2}}\left\{\frac{2 k}{k+1}\right\}^{\frac{k+1}{2(k-1)}}
$$

Eq. 6 
In above equations, ' $C_{d}$ ', ' $A_{\text {in }}$ ', ' $P_{i n}$ ', ' $T_{i n}$ ', ' $R$ ', ' $k$ ' and ' $P_{c y l}$ ' represent discharge coefficient, intake port opening area, intake pressure, intake temperature, gas constant, ratio of specific heats and in-cylinder pressure respectively. In this dissertation, a two-directional mass flow was considered. It means that the mass flowed both into and out of the combustion chamber. If the intake pressure was higher than the in-cylinder pressure, then the mass flowed from the intake port into the engine cylinder. Similarly, if the intake pressure was lower than the in-cylinder pressure, then the mass flowed from the engine cylinder into the intake port. Rewriting the same equations mentioned above for mass flow rates from the exhaust port.

$$
\begin{gathered}
\dot{m}_{\text {out }}=\frac{C_{d} A_{e x} P_{c y l}}{\left(R T_{c y l}\right)^{\frac{1}{2}}\left(\frac{P_{e x}}{P_{c y l}}\right)^{\frac{1}{k}}\left\{\frac{2 k}{k-1}\left[1-\left(\frac{P_{e x}}{P_{c y l}}\right)^{\frac{k-1}{k}}\right]\right\}^{\frac{1}{2}}} \\
\dot{m}_{\text {out }}=\frac{C_{d} A_{\text {ex }} P_{c y l}}{\left(R T_{c y l}\right)^{\frac{1}{2}}}(k)^{\frac{1}{2}}\left\{\frac{2 k}{k+1}\right\}^{\frac{k+1}{2(k-1)}}
\end{gathered}
$$

In conventional two-stroke engines, a resonant intake and a resonant exhaust system are used to improve the output power and efficiency [106]. The pressures in the intake system and the exhaust system vary according to the resonance theory. In the current numerical model, the intake pressure was assumed as a constant value for all the modeling cases considered in this investigation. However, for the numerical model validation, the intake pressure variations were inputted to the numerical model by using lookup tables. These lookup tables were obtained from the experimental system by using a pressure transducer. The exhaust pressure was assumed to be constant for both modeling and experimental results presented in this investigation.

\subsubsection{Engine combustion \& ignition model}

In this dissertation, the SI combustion mode was selected for igniting the fuel and air mixture. The engine combustion numerical model was defined by a time-based Wiebe function [107]. This timebased Wiebe function defined the mass burn fraction rate according to form factors, combustion duration, and ignition timing. These parameters were provided as an input to the numerical model. 
Moreover, the mass burn fraction profile, in turn, defined the heat addition or combustion process of the FPEG system. The mathematical equations for the time-based Wiebe function and heat release rate are provided in Eq. 8.

$$
\begin{gathered}
X_{b}=1-e^{\left(-a\left(\frac{t-t_{\text {spark }}}{t_{d}}\right)^{1+m}\right)} \\
\frac{d Q_{\text {in }}}{d t}=\eta_{H R} m_{f} L H V_{f} \frac{d X_{b}}{d t} \\
\frac{d Q_{\text {in }}}{d t}=\eta_{H R} m_{f} L H V_{f}\left(\frac{a(m+1)}{t_{d}}\left(\frac{t-t_{\text {spark }}}{t_{d}}\right)^{m}\right) e^{\left(-a\left(\frac{t-t_{\text {spark }}}{t_{d}}\right)^{1+m}\right)}
\end{gathered}
$$

Eq. 8

In Eq. 8, the terms ' $X_{b}$ ', ' $\eta_{H R}$ ', ' $m_{f}$ ', and ' $L H V_{f}$ ' represent mass burn fraction profile, combustion efficiency, the fuel mass trapped in the combustion chamber in $\mathrm{kg}$, and lower heating value of fuel in $\mathrm{J} / \mathrm{kg}$. From Eq. 8, the mass burned profile was mainly defined by four parameters (i.e. a, $\mathrm{m}$, $t_{\text {spark }}$, and $\left.t_{d}\right)$. The terms ' $a$ ' and ' $m$ ' are the form factors. These factors defined the shape of the mass burned profile. The terms ' $t_{\text {spark }}$ ', and ' $t_{d}$ ' defined the start and the duration of combustion in seconds respectively. For all the numerical modeling results, the form factors were chosen as 5 and 2 respectively. To understand the effects of these assumptions, a model sensitivity study of these parameters was presented in Chapter 4.

The fuel mass and fuel LHV values were also provided as an input to the numerical model. These values were obtained based on the mass flow rate and LHV of natural gas used in the experiments. Moreover, the combustion efficiency and combustion durations depend upon the engine operating variables. These variables include operating frequency, spark timing, compression ratio, exhaust gas retained in the cylinder, and equivalence ratio. In the numerical model, the combustion efficiency and combustion durations were considered as constant values. These values were inputted to the numerical model before the beginning of the simulation.

Since the experimental combustion data were available, a curve-fitted time-based Wiebe function was used for the numerical model validation case in Chapter 3 . The form factors and the combustion duration were calculated from the experimental mass fraction burned profile. The experimental mass fraction burned profile was calculated by normalizing the instantaneous net 
pressure with the overall net pressure rise at the end of the combustion. In any engine, the pressure difference between any two time steps was caused by two conditions. The first condition referred to the pressure difference due to a volume change between two time steps. The second condition considered the pressure difference due to combustion between two time steps. Assuming that the combustion followed a constant volume process, the pressure difference was mainly caused by the heat addition, pressure loss due to blowby, and heat transfer. Moreover, the combustion occurred only after the spark initiation in the current FPEG system. Therefore, the pressure difference at any volume was normalized by using the volume of the system at the spark initiation. Using this procedure, the net pressure and mass fraction burned at any time step are formulated below.

$$
\begin{gathered}
\Delta P_{i}=\left\{P_{i+1}-P_{i}\left[\frac{V_{i}}{V_{i+1}}\right]^{k}\right\} \frac{V_{i}}{V_{\text {spark }}} \\
P_{n e t, i}=P_{n e t, i-1}+\Delta P_{i} \\
M F B_{i}=\frac{P_{n e t, i}}{P_{n e t, f}}
\end{gathered}
$$

In the above equation, ' $i$ ' represents the current time step and ' $V_{\text {spark }}$ ' represents the cylinder volume at the instant of spark initiation. After obtaining the experimental mass fraction burned profile, the curve fitting toolbox in MATLAB® was used to calculate the form factors and combustion duration. These values were inputted to the numerical model to replicate the heat addition process of an experimental system.

Since the FPEG system was modeled for an SI combustion mode, the main important parameter to be specified was the spark or ignition timing. Variations in the spark ignition timing influenced the rise in the in-cylinder combustion pressure [108]. The variations in the combustion characteristics further caused the variations in the translator dynamics and overall system performance. Due to the absence of a crankshaft mechanism, the FPEG spark ignition timing was defined in terms of velocity after neutral point location, time or distance from BDC. In the current model, the spark timing was modeled in terms of velocity after the neutral point location. The spark signal was generated once the instantaneous translator velocity exceeded the spark trigger velocity. The spark trigger velocity was provided as an input to the numerical model. Moreover, 
the spark event in the numerical model does not include a spark delay. It was assumed as the location of Heat Release Rate (HRR) initiation.

\subsubsection{Heat transfer model}

There are no empirical models available for the heat transfer losses in the FPEG system with unique dynamics. Due to the lack of heat transfer models for the FPEG systems, the CSE heat transfer models were used in this investigation. Only convection and conduction heat transfers were utilized in the numerical model. A one-dimensional conduction heat transfer model was developed for the distribution of wall temperature based on the cylinder gas convection. In other words, the heat transfer rate was dependent only upon the gas temperature, wall temperature, and instantaneous surface area as presented in Eq. 10. The wall temperature was modeled as a function of TDC, mid-stroke, and BDC wall temperatures, and translator displacement as shown in the equation below [109]. The TDC, mid-stroke, and BDC wall temperatures were obtained from the experimental FPEG system by using K-type surface thermocouples.

$$
\begin{gathered}
\frac{d Q_{\text {out }}}{d t}=h A\left(T_{\text {gas }}-T_{\text {wall }}\right) \\
T_{\text {wall }}(x)=T_{w, T D C}+\left(4 T_{w, M i d}-3 T_{w, T D C}-T_{w, B D C}\right) \frac{x}{x_{\max }}+2\left(T_{w, T D C}+T_{w, B D C}-2 T_{w, M i d}\right)\left(\frac{x}{x_{\max }}\right)^{2}
\end{gathered}
$$

In the above equation, ' $h$ ' is the convective heat transfer coefficient. This convective heat transfer coefficient can be estimated by using multiple numerical models, namely Woschni, Annand, and Hohenberg correlations [110, 111, 112]. In the present study, a Hohenberg correlation was considered. The mathematical formulation for the Hohenberg's convective heat transfer coefficient in terms of cylinder pressure, temperature, translator velocity, and instantaneous volume is shown in Eq. 11 [112].

$$
h=a_{c} V^{-0.06} T^{-0.4} P^{0.8}\left(\frac{d x}{d t}+1.4\right)^{0.8}
$$

Eq. 11 
In the formulation above, volume, cylinder pressure, and temperature were provided in $\mathrm{m}^{3}$, bar, and $\mathrm{K}$ respectively. The term ' $h$ ' is the convective heat transfer coefficient in $W / \mathrm{m}^{2} \mathrm{~K}$. A numerical tuning coefficient ' $a_{c}$ ' (dimensionless) was also considered in the present investigation. This coefficient accounted for the effect of variable FPEG motion on heat transfer. This tuning coefficient was changed in between $80 \%$ to $120 \%$ of the CSE tuning coefficient.

\subsubsection{Piston ring frictional model}

The translator dynamics in an FPEG system are influenced by the frictional forces in the system. Therefore, various frictional submodels used in the numerical model will exhibit different translator dynamics. In an ideal FPEG system, the main frictional sources include the friction from the piston rings, seals and bearings (if any). The piston ring friction is the major source of loss of all the losses. Hence, only the piston ring friction was considered in this section. The other frictional losses like aerodynamic, springs (bearings) and seal losses were considered in the mechanical spring modeling section (i.e. Section 2.2.7).

Many FPEG researchers modeled the piston ring frictional losses either as a constant value or using a simplified equation. However, it was unclear whether they were appropriate for an accurate frictional force modeling in an FPEG system. In this numerical model, a more detailed frictional model was developed based on the hydrodynamic and asperity contact lubrication. This frictional model was based on the same models used in the CSE systems. Moreover, with the crankshaft mechanism elimination, the side thrust friction was eliminated in an ideal FPEG system. In this numerical model, an iterative based one-dimensional numerical model was developed based on Patir and Cheng's modified Reynolds hydrodynamic equation and Greenwood and Tripp's asperity contact equation [113, 114]. This one-dimensional model was solved numerically by using a MATLAB® script. A Forward-Time Central-Space (FTCS) Finite Difference Method (FDM) along with the successive over relaxation technique was used in the numerical solution. The MATLAB® script was incorporated into the existing FPEG model. For an incompressible and isothermal lubricant, the Patir and Cheng's modified Reynolds equation with the effects of surface roughness is shown in Eq. 12 [113]. This equation was solved for the pressures due to hydrodynamic contact. 


$$
\frac{d}{d x_{r}}\left(\frac{\varphi_{x} h^{3}}{\mu} \frac{d P_{h y d}}{d x_{r}}\right)=6 \dot{x} \frac{d \overline{h_{t}}}{d x_{r}}+6 \dot{x} \sigma \frac{d \varphi_{s}}{d x_{r}}+12 \frac{d \overline{h_{t}}}{d t}
$$

Eq. 12

In Eq. 12, ' $x_{r}$ ', ' $\varphi_{x}$ ', ' $\varphi_{s}$ ', ' $h$ ', ' $\bar{h}_{t}$ ', ' $\mu$ ', ' $P_{h y d}$ ', and ' $\sigma$ ' represent axial thickness of piston ring, pressure flow factor, shear flow factor, nominal oil film thickness, average local oil film thickness, lubricant oil viscosity, lubricating oil hydrodynamic pressures, and composite surface roughness of the piston ring and cylinder liner respectively. The pressure and shear flow factors compared the average pressure and lubricant flow in a rough bearing to that of a smooth bearing. These flow factors were calculated in the numerical solution based on the ratio of nominal oil film thickness and composite surface roughness i.e. $(\mathrm{h} / \sigma)$. The equations for the flow factors developed by Patir and Cheng are presented in Eq. 13 [113].

$$
\begin{array}{cc}
\varphi_{x}=1-0.90 e^{-0.56\left(\frac{h}{\sigma}\right)} & \\
\varphi_{s}=1.899\left(\frac{h}{\sigma}\right)^{0.98} e^{-0.92\left(\frac{h}{\sigma}\right)+0.05\left(\frac{h}{\sigma}\right)^{2}} & \frac{h}{\sigma} \leq 4 \\
\varphi_{s}=1.126 e^{-0.25\left(\frac{h}{\sigma}\right)} & \frac{h}{\sigma}>4
\end{array}
$$

Eq. 13

The composite surface roughness $(\sigma)$ was determined by using standard deviations of surface roughness of piston ring and cylinder liner. The surface roughness of piston ring and liner were measured experimentally by using an optical profilometer at WVU. The schematic diagram of a piston ring and cylinder liner conjunction is shown in Figure 19. The ring face profile in this investigation was assumed as a parabolic profile. The corresponding equation for the ring face profile is represented in Eq. 14.

$$
h_{x}\left(x_{r}\right)=\delta \frac{\left(x_{r}-x_{o}\right)^{2}}{\left(\frac{b}{2}+x_{o}\right)^{2}}
$$

Eq. 14 


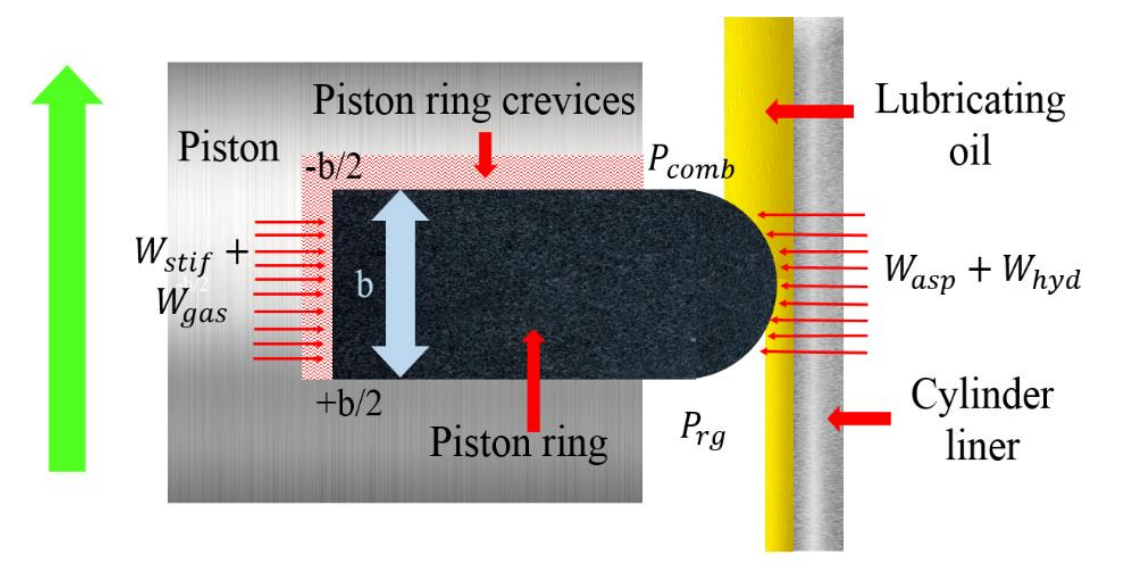

Figure 19: Schematic diagram of a piston ring and cylinder liner contact in an FPEG system.

In Eq. 14, the terms ' $h_{x}$ ', ' $\delta$ ', ' $x_{o}$ ', and ' $b$ ' represent the film thickness due to ring profile, piston ring crown height, crown offset and ring axial thickness respectively. The nominal oil film thickness was defined as a summation of film thickness due to the ring profile and minimum oil film thickness or center oil film thickness. If the ring tilt and the cylinder deformations are neglected, the equations for the nominal oil film thickness and local oil film thickness are shown in Eq. 15. In Eq. 15 , ' $\delta$ ' is the composite surface roughness and ' $f(\delta)$ ' is Gaussian probability distribution function as defined by Patir and Cheng's model [113].

$$
\begin{gathered}
h\left(x_{r}, t\right)=h_{x}\left(x_{r}\right)+h_{\min }(t) \\
\overline{h_{t}}\left(x_{r}, t\right)=\int_{-h\left(x_{r}, t\right)}^{\infty}(h+\delta) f(\delta) d \delta
\end{gathered}
$$

Eq. 15

Moreover, the lubricating oil viscosity was strongly dependent on the temperature [115]. Therefore, a relationship of lubricating oil viscosity based on Vogel's equation was considered in this investigation [115]. The relationship is shown in Eq. 16.

$$
\mu\left(T_{o i l}\right)=\mu_{o} e^{\left(\frac{a}{b+T_{o i l}}\right)}
$$

Eq. 16 
In Eq. 16, the terms ' $\mu_{o}$ ', ' $a$ ' and ' $b$ ' are the constants defined by using known values of the oil viscosity at three different temperatures. Further, the temperature of the lubricating oil $\left(T_{\text {oil }}\right)$ in ${ }^{\circ} \mathrm{C}$ was calculated as a function of translator displacement. An inlet and outlet boundary conditions were required to solve for hydrodynamic pressures in Eq. 12. The inlet boundary condition was assumed as a fully flooded condition. The fully flooded condition refers to the state where the lubricant oil flow rate is sufficient enough to obtain a fluid film region. The inlet boundary condition is shown in Eq. 17.

$$
P_{\text {hyd }}=P_{\text {in }} \quad @ x_{r}=-\frac{b}{2}
$$

Moreover, the parabolic shape of the piston ring caused the fluid film to rupture at the outlet due to cavitation. The cavitation refers to the formation of air bubbles in the oil film. This cavitation was due to rapid pressure distribution changes. Though there were many different cavitation boundary conditions, a Reynolds boundary condition was considered at the outlet when cavitation was present.

$$
\begin{gathered}
P_{\text {hyd }}=P_{\text {out }} \quad @ x_{r}=x_{c a v} \\
\frac{d P_{h y d}}{d x_{c a v}}=0
\end{gathered}
$$

In certain conditions, especially at the dead centers, the squeeze film effect dominated the pressure distribution. In other words, there was no cavitation at the outlet due to the squeeze film effect. Under such conditions, a non-cavitation boundary condition as shown below was used as an outlet boundary condition.

$$
P_{\text {hyd }}=P_{\text {out }} \quad @ x_{r}=+\frac{b}{2}
$$

In Eq. 17, 18, and 19, the terms ' $P_{\text {in }}$ ', ' $P_{\text {out }}$ ' and ' $x_{\text {cav }}$ ' denote inlet pressure, outlet pressure, and cavitation locations respectively. The inlet and outlet pressures were varied depending upon the 
stroke direction. The stroke direction in the numerical model was defined based on the sign of translator velocity. For an expansion stroke as shown in Figure 19, the inlet and outlet pressures were combustion and inter-ring pressures respectively. For a compression stroke, the inlet and outlet pressures were inter-ring and combustion pressures. In addition to these boundary conditions, an arbitrary minimum oil film thickness was considered as an initial condition. The initial condition is shown in Eq. 20.

$$
h\left(x_{r}, t=t_{i}\right)=h_{x}\left(x_{r}\right)+h_{\min }\left(t_{i}\right)
$$

Eq. 20

If the ratio of nominal oil film thickness to composite surface roughness is relatively small, then the friction is said to be in a mixed lubrication regime [113]. In this lubrication regime, the surface peaks of both cylinder liner and piston rings were in contact with each other. As a result, the load was carried by both contacting asperities and hydrodynamic lubricant film. During motoring, the hydrodynamic lubrication regime dominated the entire engine cycle. This was due to the lower oil temperatures during motoring when compared to the combustion conditions. Under firing conditions, the gas pressures and temperatures reduced the viscosity of the oil. Therefore, a mixed lubrication regime dominated the entire engine cycle.

If the $\mathrm{h} / \sigma$ value were greater than 4 , then the regime was considered as a hydrodynamic regime. Else the regime was considered as a mixed lubrication regime. An asperity contact model was defined by using Greenwood and Tripp's rough surface model [114]. According to Greenwood and Tripp's rough surface model, the asperity contact pressure distribution is presented in Eq. 21 .

$$
\begin{gathered}
P_{a s p}=\frac{16 \sqrt{2}}{15} \pi(\eta \beta \sigma)^{2} \sqrt{\frac{\sigma}{\beta}} E F_{2.5}\left(\frac{h}{\sigma}\right) \\
E=\frac{1}{\left(\frac{1-\vartheta_{r g}^{2}}{E_{r g}}+\frac{1-\vartheta_{c y l}^{2}}{E_{c y l}}\right)} \\
F_{2.5}\left(\frac{h}{\sigma}\right)=\frac{1}{\sqrt{2 \pi}} \int_{\frac{h}{\sigma}}^{\infty}\left(s-\left(\frac{h}{\sigma}\right)\right)^{2.5} e^{-\frac{s^{2}}{2}} d s
\end{gathered}
$$

Eq. 21 
In Eq. 21, ' $P_{a s p}$ ', ' $\beta$ ', ' $\eta$ ', ' $E$ ', ' $E_{r g}$ ', ' $E_{c y l}$ ', ' $\vartheta_{r g}$ ', ' $\vartheta_{c y l}$ ' and ' $F_{2.5}\left(\frac{h}{\sigma}\right)$ ' represent asperity contact pressure, asperity curvature radius, number of asperities per unit contact area, composite Young's modulus, piston ring Young's modulus, cylinder liner Young's modulus, Poisson's ratio of piston ring and cylinder liner, and Gaussian probability distribution function for the asperity heights respectively. Since the ring tilt and ring rotation were neglected, the forces acting on the piston ring at any instant were radial in nature. From Figure 19, the net resultant of forces due to asperity and hydrodynamic contact were in equilibrium with the mechanical ring expansion force and gas force at all times. The corresponding load equation is shown in Eq. 22.

$$
W_{\text {asp }}+W_{\text {hyd }}=W_{\text {stiff }}+W_{g a s}
$$

Eq. 22

In Eq. 22, ' $W_{\text {asp }}$ ', ' $W_{\text {hyd }}$ ', ' $W_{\text {stiff }}$ ' and ' $W_{\text {gas }}$ ' refer to asperity contact load, hydrodynamic load, mechanical ring expansion force, and gas forces behind the piston ring. The asperity contacts and hydrodynamic loads were calculated from asperity contact and hydrodynamic pressures. The mechanical ring expansion and gas forces were provided as an input to the model at every time step. The respective equations for the asperity load, hydrodynamic load and gas forces are provided in Eq. 23.

$$
\begin{gathered}
W_{a s p}(t)=\int_{\frac{-b}{2}}^{\frac{b}{2}} P_{a s p}\left(x_{r}, t\right) d x_{r} \\
W_{\text {hyd }}(t)=\int_{\frac{-b}{2}}^{\frac{b}{2}} P_{h y d}\left(x_{r}, t\right) d x_{r} \\
W_{\text {gas }}=b\left(\max \left(P_{\text {in }}, P_{\text {out }}\right)-\min \left(P_{\text {in }}, P_{\text {out }}\right)\right)
\end{gathered}
$$

Eq. 23

The frictional force from the piston ring and cylinder liner contact comprised of frictional force from hydrodynamic and asperity contacts. The frictional forces due to these contacts are shown in Eq. $24[113,114]$. 


$$
\begin{gathered}
\tau_{h y d}\left(x_{r}, t\right)=\frac{\mu U_{p}}{h}\left(\varphi_{f}+\left(1-\left(\frac{\sigma_{c y l}}{\sigma}\right)^{2}\right) \varphi_{f s}\right)+\frac{d P_{h y d}}{d x_{r}}\left(\frac{h \varphi_{f p}}{2}+\left(h \varphi_{f p}-\bar{h}_{t}\right)\left(\frac{\sigma_{c y l}}{\sigma}\right)^{2}\right) \\
F_{h y d}(t)=\int_{\frac{-b}{2}}^{\frac{b}{2}} \tau_{h y d}\left(x_{r}, t\right) d x_{r} \\
F_{a s p}(t)=\tau_{o} A_{a s p}+\gamma W_{a s p} \\
F_{\text {ring }}(t)=F_{h y d}(t)+F_{a s p}(t)
\end{gathered}
$$

The terms ' $\varphi_{f}$ ', ' $\varphi_{f p}$ ' and ' $\varphi_{f s}$ ' in the above equation represent oil shear stress factors, whereas ' $\tau_{\text {hyd }}$ ' is hydrodynamic shear stress. These factors were dependent upon minimum oil film thickness and composite surface roughness. The terms ' $\tau_{o}$ ' and ' $\gamma$ ' represented surface asperity shear strength and rate of shear stress changes. Finally, the total frictional force was calculated by the summation of the hydrodynamic and asperity friction force.

The step by step procedure for the calculation of frictional force due to piston ring contact with the cylinder liner is presented below. The corresponding flow chart is shown in Figure 20.

Step 1: The procedure was started with an assumption of initial minimal oil film thickness. The combustion pressures, interring pressures and initial translator dynamics were provided as an input to the numerical model.

Step 2: The lubricating oil temperature was provided as an input at mid-stroke, TDC, and BDC locations. The lubricating oil viscosity was calculated based on Eq. 16.

Step 3: The piston ring axial thickness was divided into 200 nodes. The nominal and average oil film thicknesses were calculated at an initial time step. The pressure and shear flow factors were also calculated based on the ratio of nominal oil film thickness and composite surface roughness. Step 4: For the first time step, Eq. 12, and Eq. 21 were solved for hydrodynamic and asperity pressures by using an FDM method.

Step 5: After solving Eq. 12, the hydrodynamic pressure convergence condition was checked by the formula given below. 


$$
\frac{\sum_{i=1}^{N}\left|P_{h y d}^{n}-P_{h y d}^{n-1}\right|}{\sum_{i=1}^{N}\left|P_{h y d}^{n}\right|} \leq 10^{-6}
$$

Eq. 25

In Eq. 25, the terms ' $n$ ' and ' $i$ ' refer to iteration number and nodes. If the above condition was not met, the hydrodynamic pressures were modified by using the formula below.

$$
P_{h y d}^{n}=\chi P_{h y d}^{n}+(1-\chi) P_{h y d}^{n-1}
$$

In the above equation, ' $\chi$ ' is a successive overrelaxation factor of 1.4 .

Step 6: Once the hydrodynamic and asperity pressures were obtained, the asperity contact, and hydrodynamic loads were calculated based on Eq. 23.

Step 7: Later, the solution was checked for the load convergence criterion by using the following condition.

$$
\left|\frac{W_{a s p}+W_{h y d}-W_{s t i f f}-W_{g a s}}{W_{a s p}+W_{h y d}}\right| \leq 10^{-5}
$$

Eq. 27

If the condition was not met, the minimum oil film thickness was modified by using the following expression. Later, the procedure was repeated until the load convergence condition was satisfied.

$$
h_{\text {min }}^{n}=h_{\text {min }}{ }^{n-1}+0.000005\left(\frac{W_{\text {asp }}+W_{\text {hyd }}-W_{\text {stiff }}-W_{\text {gas }}}{\max \left(W_{\text {asp }}+W_{\text {hyd }}, W_{\text {stiff }}+W_{\text {gas }}\right)}\right) h_{\text {min }}{ }^{n-1}
$$

Eq. 28

Step 8: After the load convergence criterion, the oil film thickness was estimated for the next time step by using Euler's method. Later, the procedure was repeated for one operating cycle.

Step 9: Since the model started with an initial condition, the initial minimum oil film thickness was also checked with a convergence criterion.

$$
\left|\frac{h_{\min }\left(t=t_{\text {end }}\right)-h_{\min }\left(t=t_{i}\right)}{h_{\min }\left(t=t_{\text {end }}\right)}\right| \leq 10^{-4}
$$

Eq. 29 
If not, these steps were repeated again with a modified initial minimum oil film thickness.

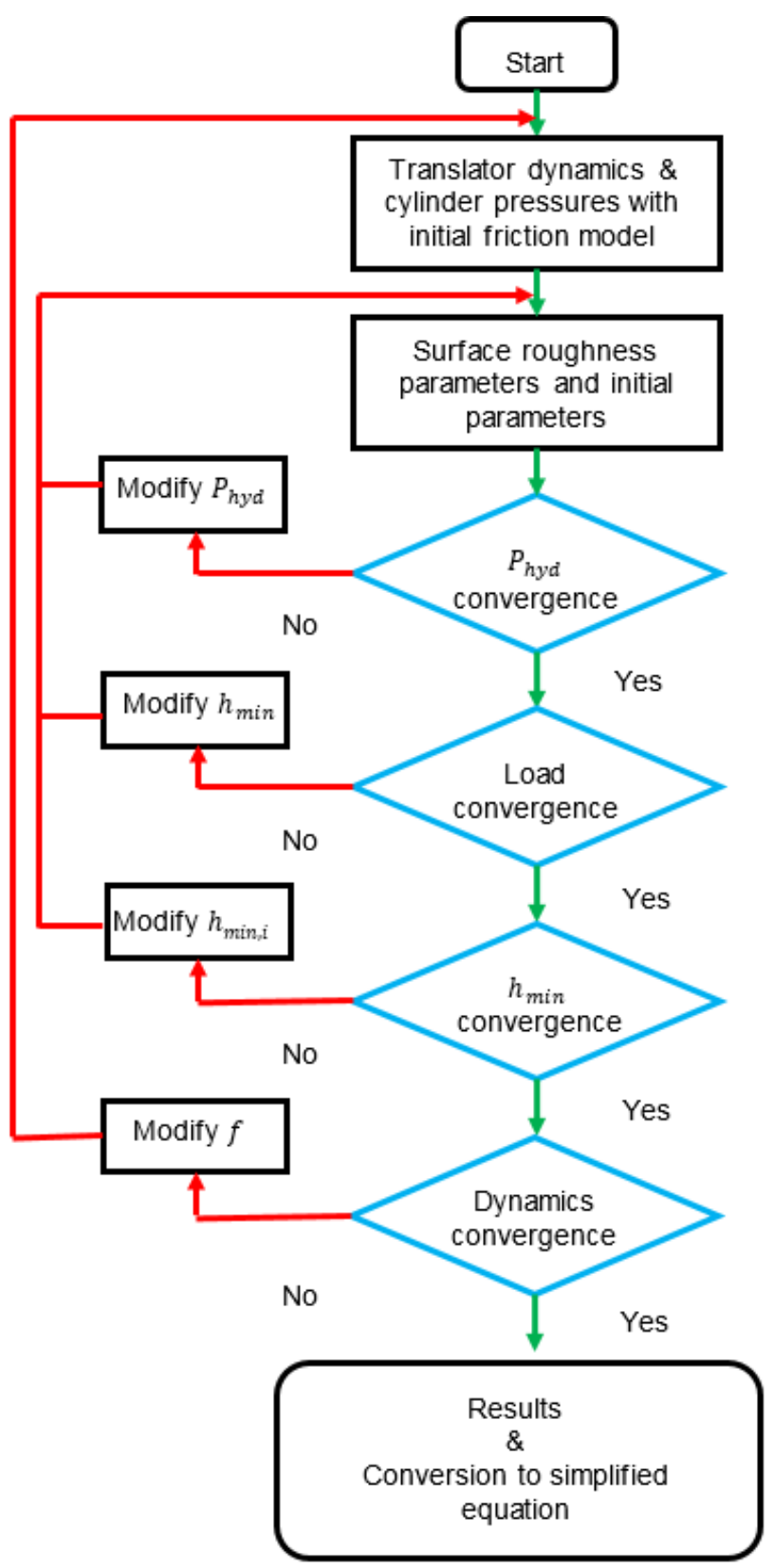

Figure 20: Flow chart for the minimum oil film thickness calculation of piston ring in an FPEG system.

Step 10: Finally, the solution was checked for the translator dynamics convergence condition. If the condition was not satisfied, the frictional coefficient was modified until the translator dynamics 
were converged. This piston ring frictional model was incorporated into the existing model and was executed at every time step.

\subsubsection{Mechanical springs model}

In this dissertation, stiff springs were considered in the final FPEG design at both modeling and experimentation level. These stiff mechanical flexure springs acted as energy storage devices within the system. Moreover, the addition of stiff springs reduced cycle-to-cycle variations and improved electric power and operating frequency. At the modeling level, these flexure springs were modeled as linear deflection springs with a constant spring stiffness and damping coefficient as shown in Eq. 30.

$$
F_{s p r}=-k x-\beta \frac{d x}{d t}
$$

In the above equation, the terms ' $k$ ' and ' $\beta$ ' represent the spring stiffness and damping coefficient. respectively. The damping coefficient was obtained by carrying out a ring-down test on the experimental FPEG system. The ring-down test refers to the removal of a LEM motor force once the translator displacement reached a prescribed value. With the absence of an external force, the peak displacement reduced in the next consecutive cycles. This was due to spring damping. After certain time, the system reached to a stall. Moreover, the cycle-to-cycle peak displacement followed an exponential curve with an associated exponential coefficient. This coefficient was termed as the damping coefficient. The value of the damping coefficient was obtained by exponential curve fitting the peak displacement.

In the current dissertation, the damping coefficient also considered the copper and aerodynamic losses without the piston ring losses. This was because the ring-down tests were performed on the FPEG system without an engine cylinder. As a result, the damping coefficient was dominated by the external losses in the system. The external losses were aerodynamic, seal, and bearing losses. However, in reality, the damping coefficient was very low for the springs if they were considered alone. The spring alone damping coefficient was obtained by testing the springs individually in a separate test setup. Since the spring model interacted with other models, the damping coefficient with all the system losses was considered in defining the spring forces. 


\subsubsection{LEM motor \& generator model}

In the Simulink environment, the LEM model was developed by using a switch case subsystem model. With the conditional statement defined in the controller system in Section 5.1, the LEM model was switched between the motor and generator modes. More details regarding these submodels are shown in the LEM functional blocks presented in Section 2.2.9. This section only considered the corresponding mathematical equations used to define the LEM model.

During the generating mode, the LEM was modeled by using equivalent circuit parameters based on an open-circuit voltage [116]. The LEM generator modeled as an equivalent circuit is shown in Figure 21. The equivalent circuit model represented the current flowing through the LEM inductance, LEM resistance, and external load resistance. The total voltage, impedance and current flowing in the equivalent circuit are written in Eq. 31 [116].

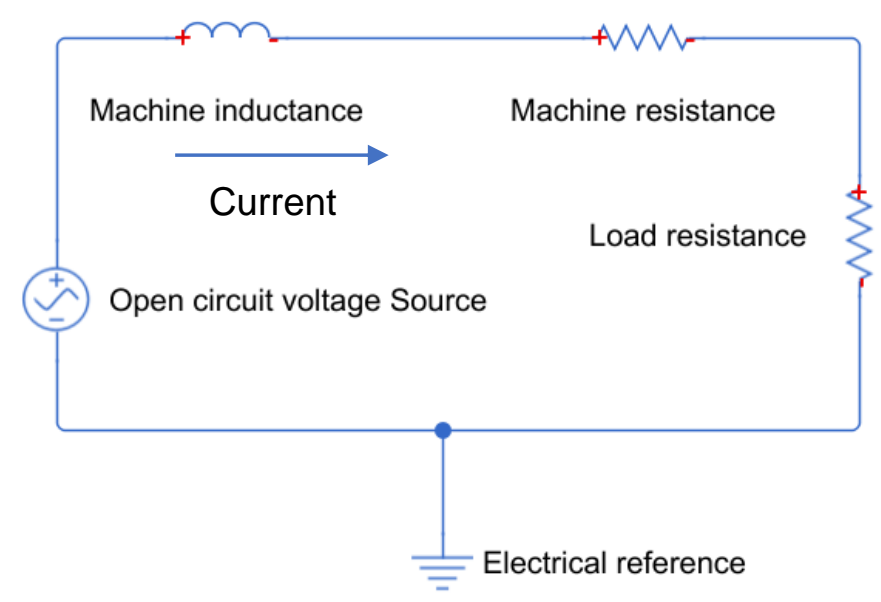

Figure 21: Equivalent circuit diagram of a LEM generator model.

$$
\begin{gathered}
V_{\text {open }}=V_{R}+V_{L}+V_{\text {Load }} \\
|Z|=\sqrt{\left(\left(R_{L E M}+R_{\text {Load }}\right)^{2}+(2 \pi f L)^{2}\right)} \\
i_{\text {Load }}=\frac{V_{\text {open }}}{|Z|} \\
V_{\text {Load }}=i_{\text {Load }} * R_{\text {Load }}
\end{gathered}
$$




$$
P_{L E M}=V_{\text {Load }} * i_{\text {Load }}
$$

In Eq. 31, the terms ' $V$ ', ' $i$ ', ' $Z$ ', ' $P$ ', ' $L$ ' and ' $f$ ' represent voltage, current, impedance, power, inductance, and operating frequency. The subscripts 'Open' and 'Load' represent open-circuit and external load respectively. In order to improve the power factor and to compensate the reactive inductance power, a capacitor was also added to the external load. This capacitor can be connected either in series or in parallel to the external load resistance. With the capacitors added in the circuit, the same equations except the impedance equation in Eq. 26 were used to calculate the power. The impedance equations when the capacitors connected in series and parallel are shown in Eq. 32.

$$
\begin{gathered}
|Z|=\sqrt{\left(\left(R_{L E M}+R_{\text {Load }}\right)^{2}+\left(2 \pi f L-\frac{1}{2 \pi f C}\right)^{2}\right)} \quad \text { (series) } \\
|Z|=\sqrt{\left(\left(R_{L E M}\right)^{2}+\left(2 \pi f L-\left(\frac{R_{\text {Load }}}{2 \pi f C R_{\text {Load }}+1}\right)\right)^{2}\right)} \quad \text { (parallel) }
\end{gathered}
$$

Eq. 32

By combining the equations provided in Eq. 26 and Eq. 27, the LEM submodel calculated the voltage, current, and power in the LEM coil windings. The LEM forces were obtained by calculating a transfer function with either voltage or current as an input.

\subsubsection{MATLAB®/Simulink/ Simscape integration}

In this research investigation, a component-based numerical model was developed in $M A T L A B \circledast /$ Simulink for single and dual cylinder FPEG systems. This component-based modeling allowed for the easy interchangeability of various submodels used in the FPEG plant system. Moreover, a hierarchical based approach containing multiple layers was considered in modeling the system. For example, an engine cylinder functional block contained a subsystem block at the highest level. It received inputs from dynamics, and controller blocks and provided an output of cylinder characteristics. At the next hierarchical layer, it contained a set of blocks representing 
the calculations for cylinder pressure, temperature, species management, and gas exchange. This section graphically characterized the numerical model architecture developed in the Simulink environment. The representation of the Simulink model at its highest level is shown in Figure 22.

At the highest level, the FPEG system signals were fed into three subsystems. The first subsystem, i.e. 'FPEG plant system', consisted of functional blocks for all the FPEG components. It included functional blocks of an engine cylinder, mechanical springs, LEM, friction, and translator dynamics. The second system, i.e. 'Controller system', provided the control strategies for the FPEG plant system. This controller subsystem allowed the system to operate steadily for a wide range of operating conditions. The third subsystem, i.e. 'Data logging' subsystem, sent the simulation data to the MATLAB® workspace. The simulation data was further post-processed for graphical visualization of the modeling results. Only the FPEG plant and controller subsystems provided the data to the FPEG system. The data logging subsystem delivered no output information. This section explained only about the FPEG plant system. The controller subsystem architecture was explained comprehensively in Chapter 5 of this document.

The next hierarchical level of the FPEG plant subsystem is shown in Figure 23. As shown in Figure 23, the FPEG plant model included an LEM, engine cylinder, mechanical springs, translator dynamics, and system friction subsystems. Every subsystem was color-coded for easy visualization and understanding. For example, the cylinder outputs and translator dynamics were represented in green and blue colors respectively. The same color coding was applied throughout the numerical model. The FPEG plant system received the cylinder characteristics from the previous time step and control signals from the controller subsystem. The plant system provided the information related to translator dynamics, friction, LEM, springs, and engine operation. Simscape libraries were utilized for modeling the spring, LEM, and frictional systems. An equation-based Simulink approach was used for modeling the engine cylinder and translator dynamics systems. The major difference between these two approaches was that the Simscape diagrams represent an actual physical system layout. In other words, the Simscape library provided the components (i.e. springs, resistors, and capacitors) to be used in the numerical model directly. This Simscape approach allowed for modeling the systems rigorously. The rigorous development was obtained by connecting the physical library components directly. The connection ports of Simscape and Simulink were non-directional and directional respectively. 
Moreover, the Simscape connection ports allowed an energy transfer between the physical components.

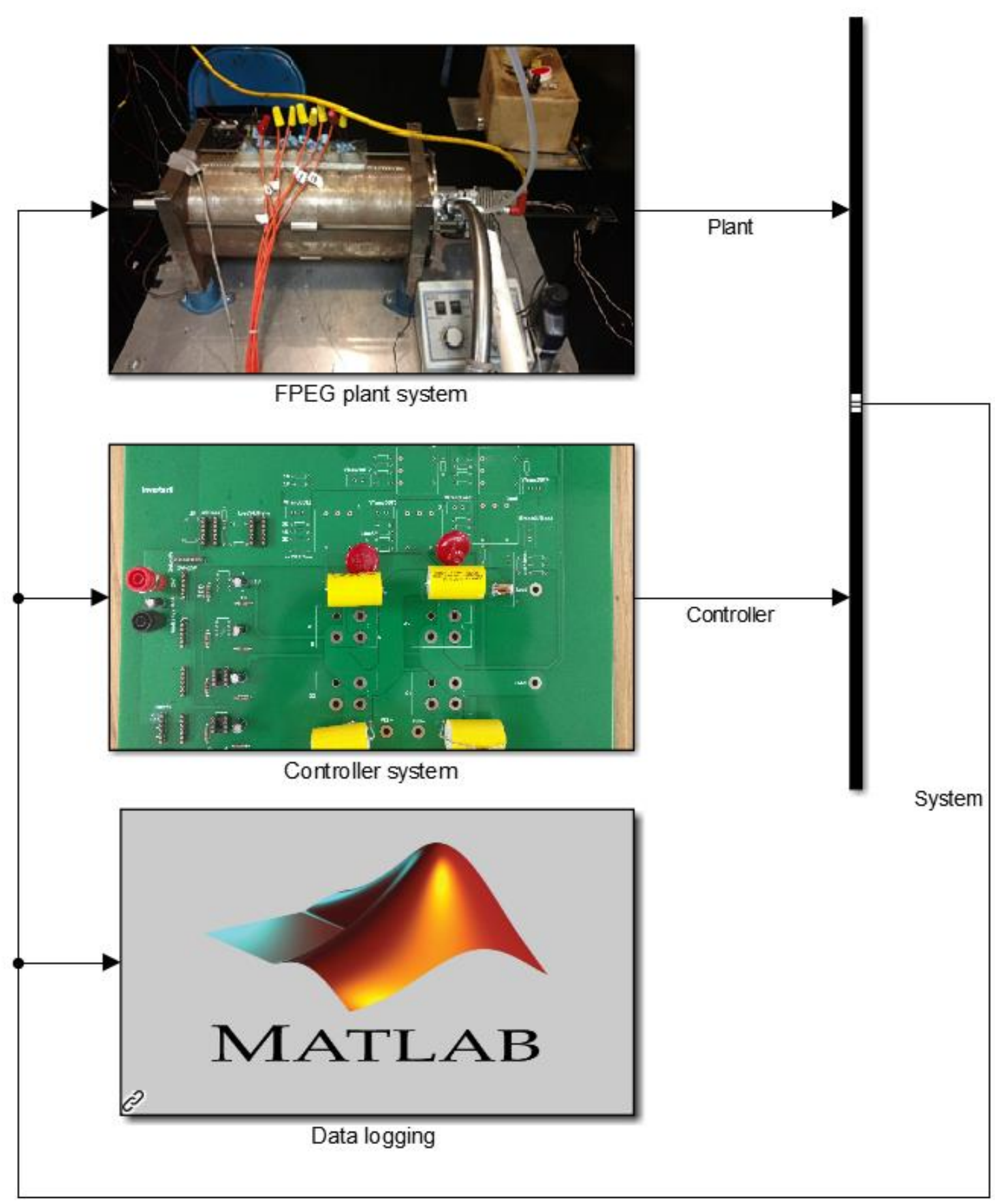

Figure 22: MATLAB®/ Simulink numerical model architecture of the FPEG system at the highest level. 

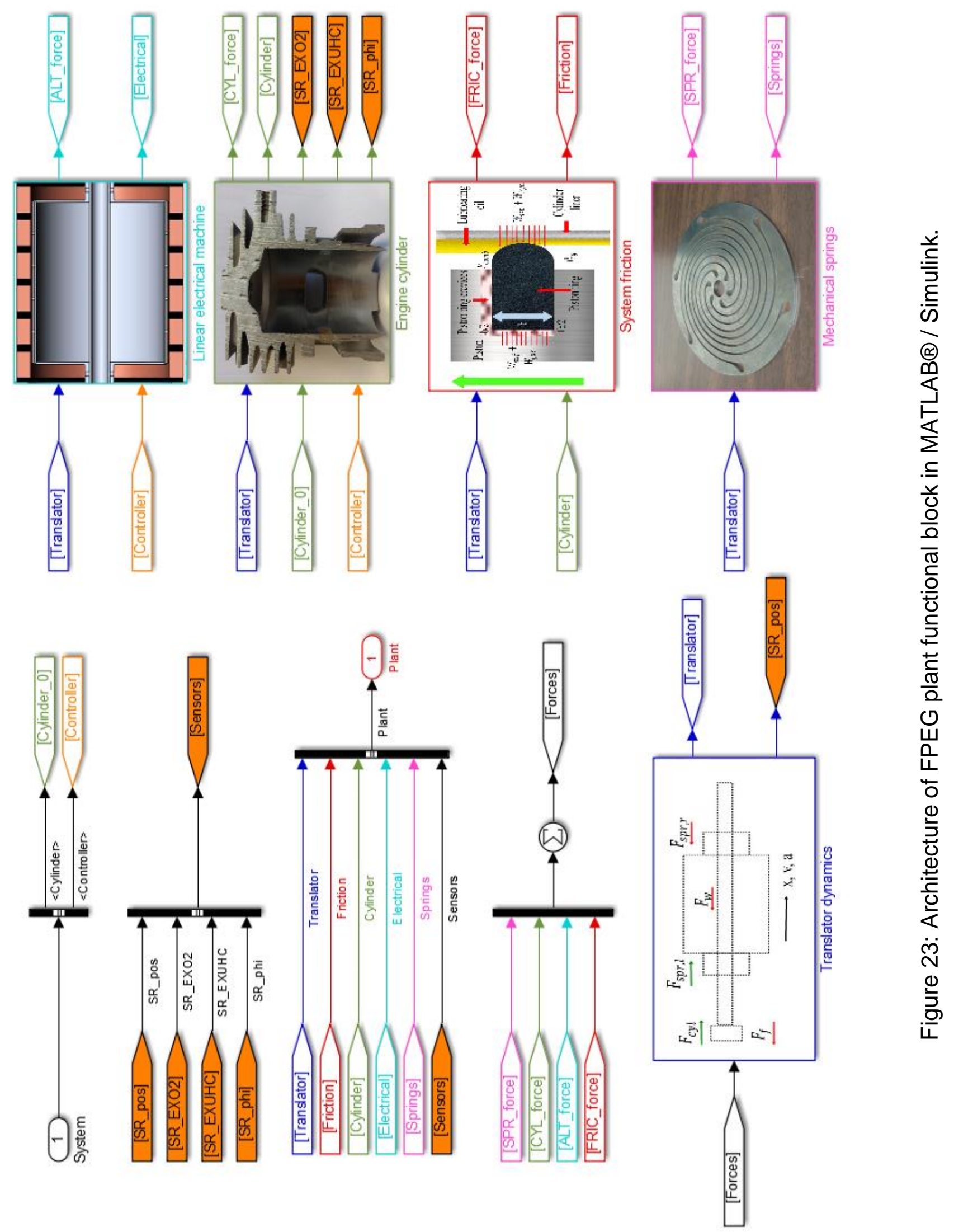
The equation-based Simulink approach represented the mathematical equations graphically. In other words, the models were developed based on the mathematical equations and logical operators in Simulink. Moreover, the signal routing in Simulink was directional in nature. As seen in Figure 23, the FPEG plant model comprised of five subsystems, namely 'Translator dynamics', 'Linear electric machine', 'Engine cylinder', 'System friction', and 'Mechanical springs' as shown in Figure 23. The schematic representation of the 'Translator dynamics' submodel is shown in Figure 24.

The system of forces acting on the translator rod was provided as an input to the translator dynamics subsystem. With the information of forces and translator moving mass, the system calculated the translator position, velocity, and acceleration. These parameters were obtained by using a second order integrator block. The state diagram 'Translator State' used a logic-based decision approach to define the state of the translator. In other words, the state diagram represented the transition of translator from one state to another state. By using translator velocity, the translator state diagram defined the TDC and BDC locations. These locations were defined based on the sign of translator velocity. Moreover, the time between two consecutive piston reversal points (i.e. sign changes in the translator velocity) calculated the resonance or operating frequency of the system. The TDC, BDC, and frequency calculations were further processed to calculate port opening and closing locations, trapped compression ratio, stroke length, and stroke direction.

The architecture of the LEM subsystem model at its highest level is shown in Figure 25. The LEM subsystem received the inputs from translator dynamics and controller subsystems. The controller subsystem primarily provided an input related to the operating mode of the LEM as seen in Figure 25. In addition to that, the controller also provided information of motoring or resonant frequency and generator gain. The motoring frequency was used to calculate the duration and initiation of the motoring force. In contrast, the generator gain scaled the LEM power output according to the translator dynamics for a stable FPEG operation. 


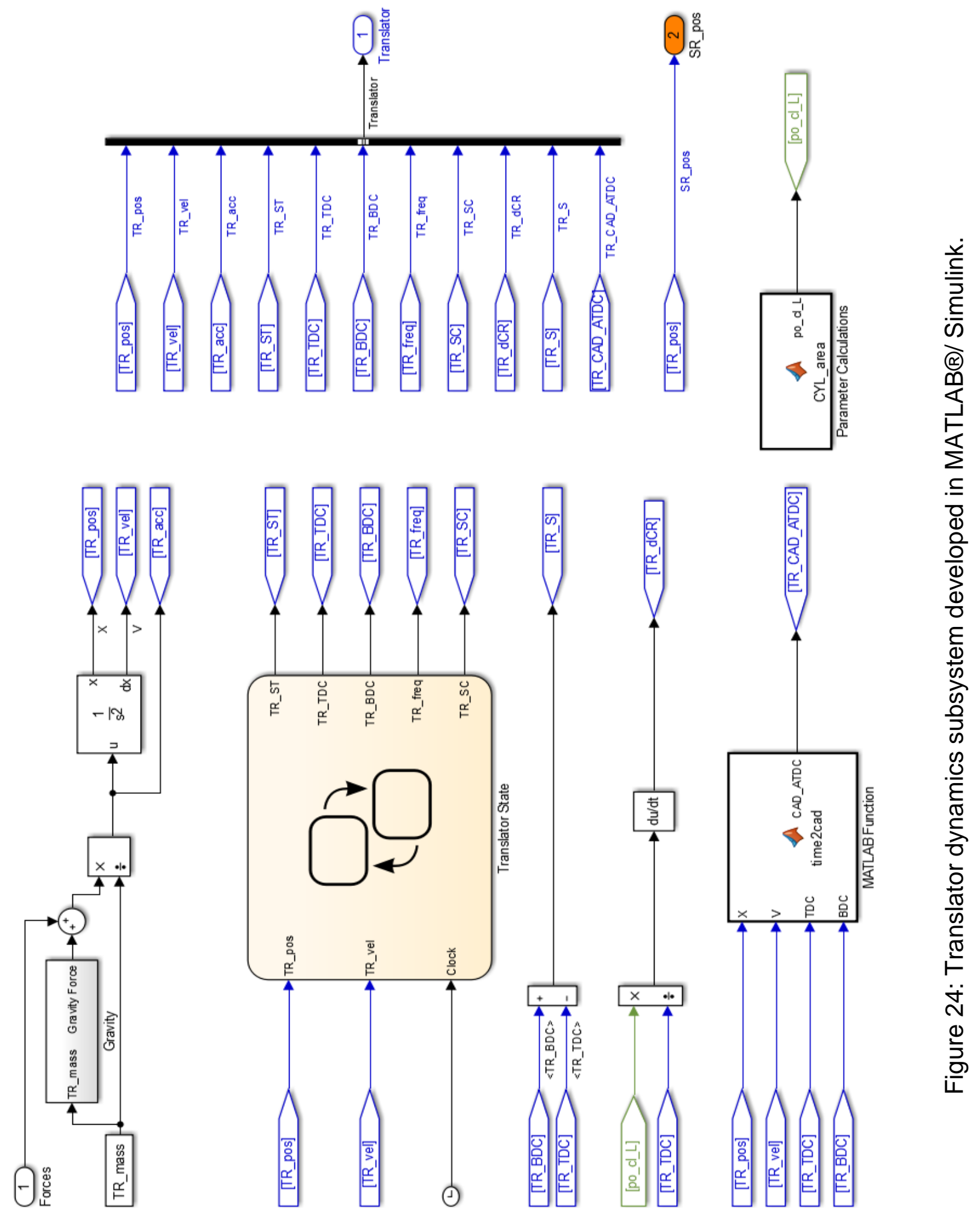


As seen in Figure 26, an $\mathrm{H}$-bridge circuit was designed for controlling the input motoring force. The H-bridge circuit consisted of four Insulated Gate Bipolar Transistor (IGBT) systems arranged in two legs. This two leg separation helped in changing the polarity of motoring forces depending upon the stroke direction. When the two IGBT's on the left were turned on, a positive voltage was applied to the FPEG system. Similarly, when the IGBT's on the right were turned on, a negative voltage was applied to the FPEG system. Since the current FPEG system considered stiff mechanical springs, only two IGBT's were operated at any time. The return force was supplied by the springs under the expansion process. In other words, the $\mathrm{H}$-bridge circuit provided only a positive motoring force for the compression of mechanical springs.

The main important process in the operation of the IGBT's was the governance of the pulse width. The pulse width acted as a control for switching the IGBT's. This pulse width was generated by using a pulse width modulator. The duration of the pulse width was defined as a percentage of motoring frequency. Since the FPEG system considered stiff springs, the initial motoring frequency was calculated based on the magnitudes of spring stiffness and translator moving mass.

In summary, based on the initial starting position, the controller initially calculated the direction of the translator movement (i.e. compression or expansion stroke). Next, the controller identified the dead center locations by using sign changes in velocity. Finally, the controller calculated the motoring frequency. This value was obtained based on the duration between two consecutive sign changes. With the information of dead center locations and motoring frequency, the LEM was pulsed at the resonant frequency to achieve the optimized motoring performance. For all the cases, the IGBT's were turned on exactly at TDC. Moreover, these IGBT's were turned on for $35 \%$ of the total cycle time or $70 \%$ of the total stroke. The duty cycle value (35\%) was provided as a numerical input to the model. This value can be seen on the top left in Figure 26.

Figure 27 shows the modeling of an LEM generator based on equivalent circuit parameters. Initially, the translator velocity was converted into an open-circuit voltage. This was done by using a translational electro-mechanical converter block in Simscape. The coefficient for obtaining the open-circuit voltage was provided as an input to the electro-mechanical converter block. This coefficient was obtained from the experimental LEM system. Moreover, different LEM designs had different open-circuit voltage coefficients. 

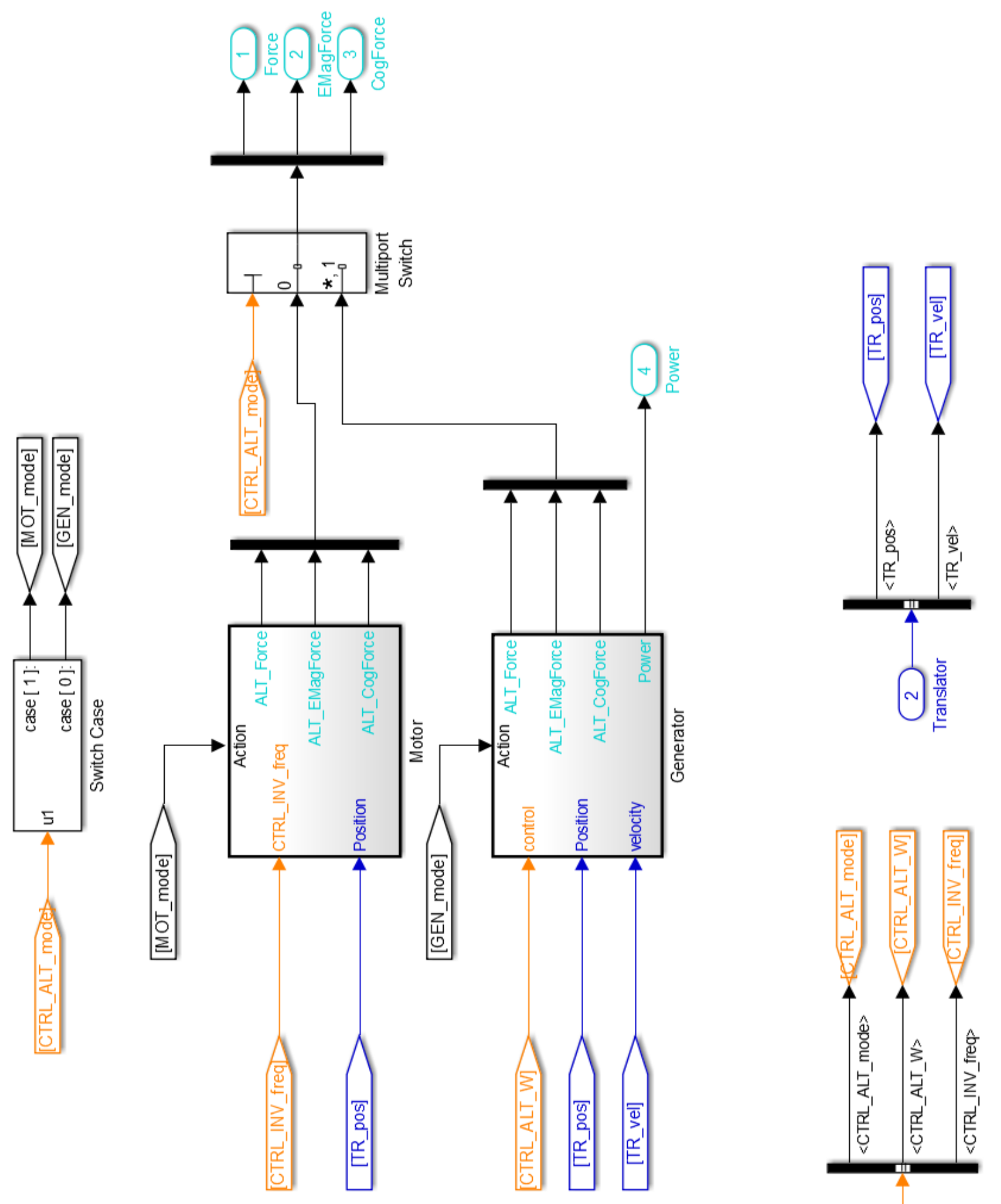

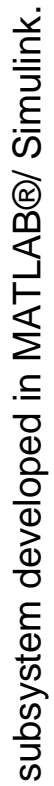

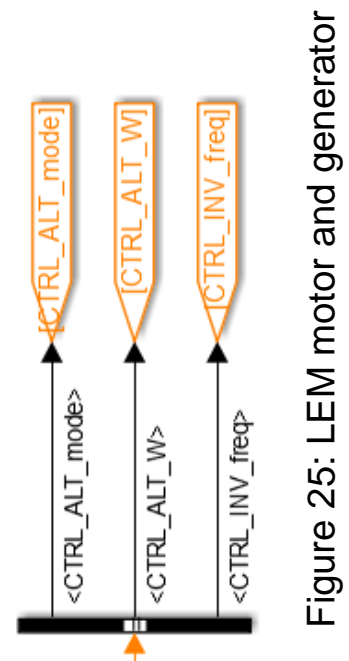

Ө亨 


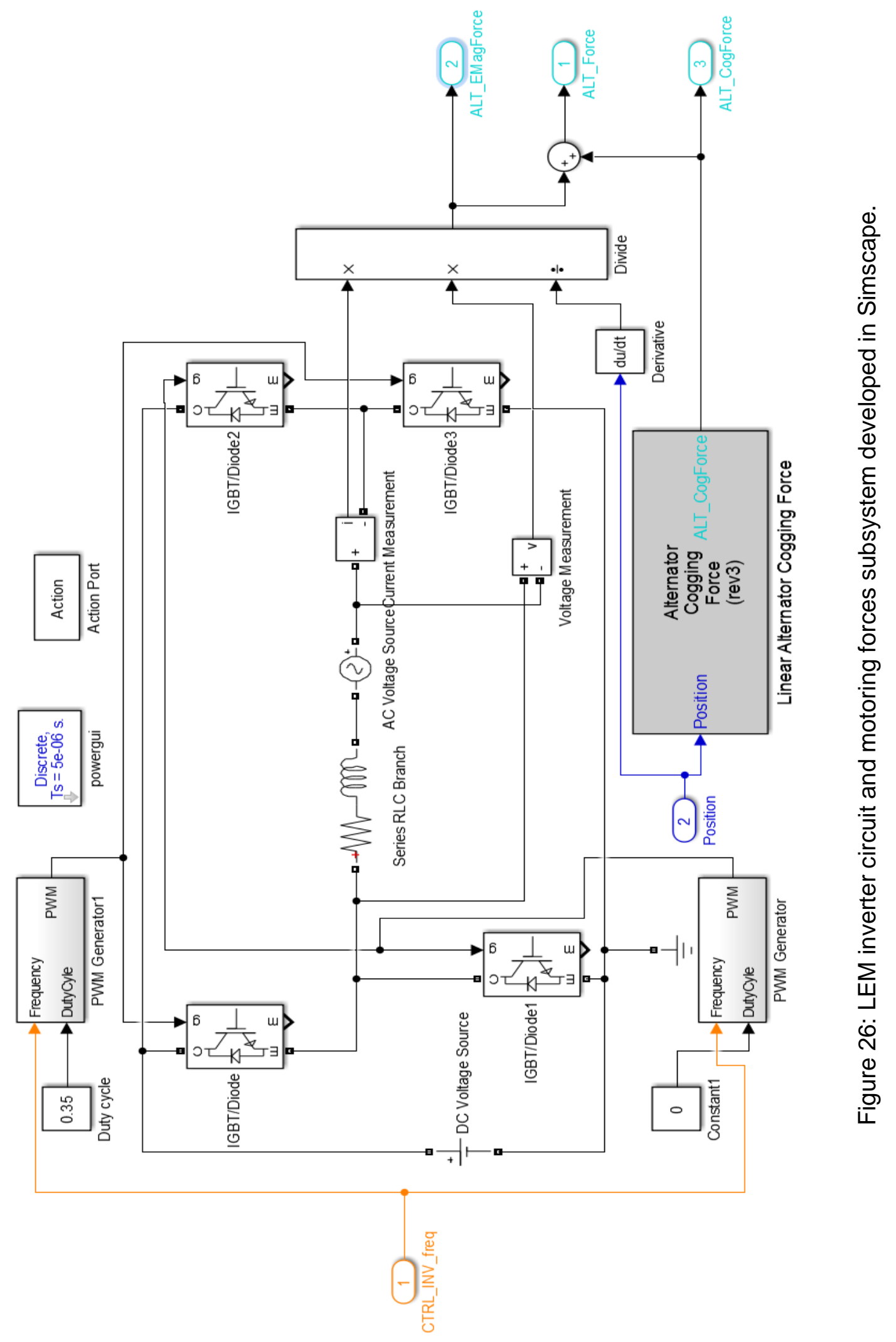


The LEM machine was modeled as an R-L circuit. The LEM machine resistance and inductance values were provided as an input to the numerical model. Finally, the load resistor and capacitors were added to the circuit. In Figure 27, the load resistor and capacitors were connected in parallel. The LEM generator submodel provided the information of voltage and current generated in the windings. These values were further used to calculate the electromagnetic force and electrical power output.

Of all the subsystems, the development of an engine cylinder model was the most complicated. The calculation of engine cylinder force requires the calculation of in-cylinder pressure. This cylinder pressure rise is a function of heat addition, heat rejection, and flow energy flowing into and out of the system. The subsystem for calculating the in-cylinder pressure is shown in Figure 28. The cylinder pressure system received the inputs from the previous state of the cylinder, heat transfer rate, heat addition rate, mass flow rates of inlet and outlet species. Using these inputs, the cylinder pressure subsystem calculated the cylinder pressure and inlet and outlet enthalpies using an open system energy balance equation. Figure 29 shows different subsystems of heat addition, and heat rejection used for the calculation of in-cylinder pressure. These subsystems were developed according to the mathematical equations provided in the previous section.

The system frictional model in the numerical model was divided into three components. The first component dealt with the piston ring-cylinder liner contact frictional force. It was modeled as a function of the cylinder, inter-ring, and crankcase compression pressures. If the FPEG system had only one piston ring, then the crankcase compression pressure was considered as the pressure below the ring. If the FPEG system had two piston rings, then the frictional model calculated the inter-ring pressure. In other words, the frictional model was modeled for both one and two piston rings. The ring number was provided as an input to the numerical model. In case of a two piston ring system, two different frictional coefficients were calculated by the piston ring friction model. The second term calculated the friction force due to a mechanical ring expansion force. Finally, the third term calculated the viscous force from the lubricating oil. 


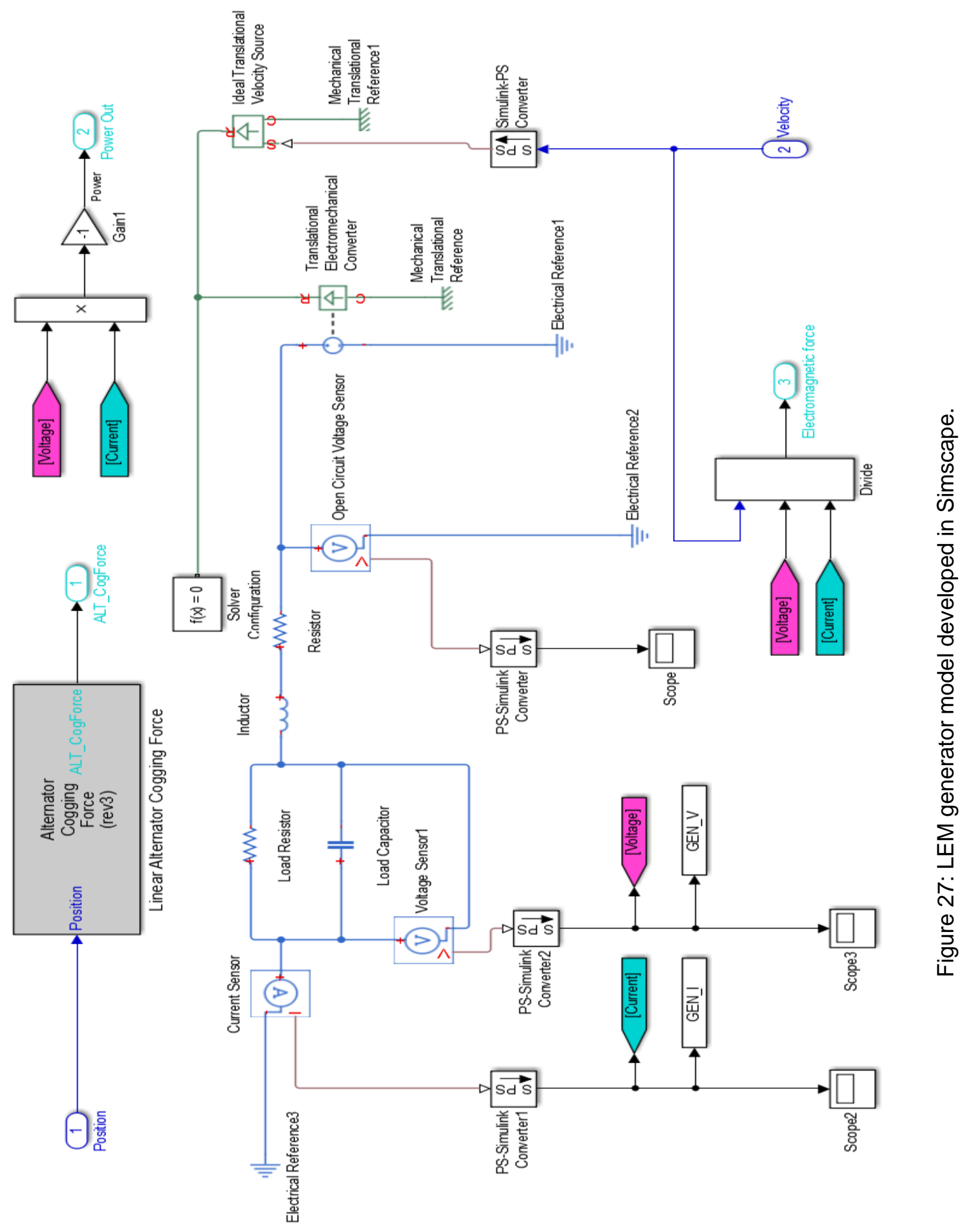


The frictional forces from all other sources in the system were considered in the damping coefficient of the springs. This was because of their minimal magnitude when compared to the piston ring friction forces. The calculation of the friction coefficient was a complex process. It was influenced by the number of piston rings, type of lubrication, piston ring geometry, piston ring radial force, and in-cylinder pressure. The frictional coefficient $(f)$ used in this study was tuned by using the mixed hydrodynamic lubrication model developed in MATLAB®. The model was solved numerically by using the FDM. The corresponding equation is provided in Eq. 28 and the Simulink architecture is shown in Figure 30.

$$
F_{f}=\operatorname{sign}(\dot{x})\left(f\left(\pi b B\left(P_{c y l}-P_{r g}\right)+F_{r g}\right)+c_{f, d y n} \dot{x}\right)
$$

Eq. 28

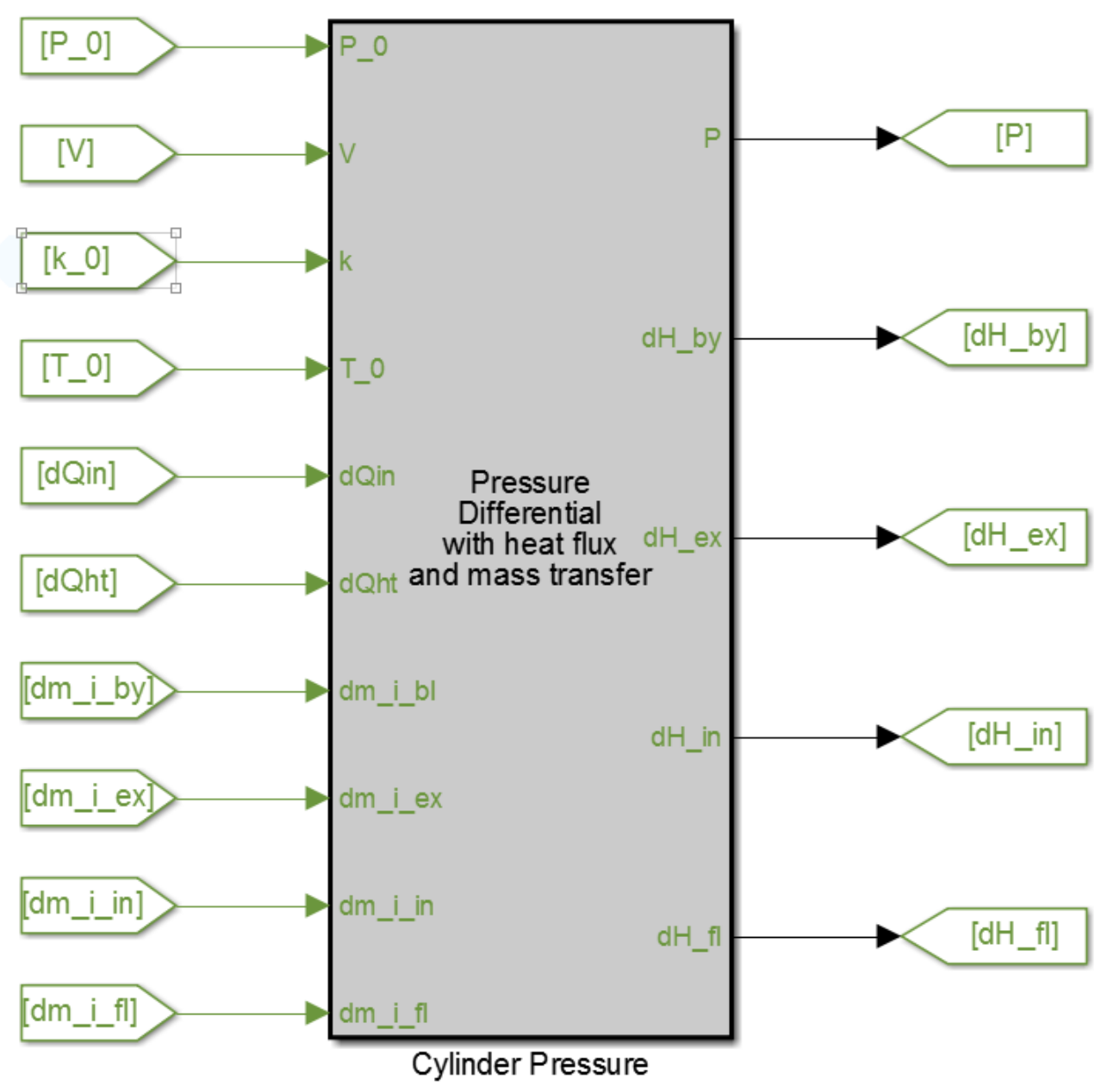

Figure 28: Cylinder pressure subsystem model developed in MATLAB®/ Simulink. 
Finally, the formulation of the mechanical spring forces is shown in Figure 31 . The spring subsystem received translator displacement as an input. It provided the spring force as an output for a given spring stiffness and damping coefficient.
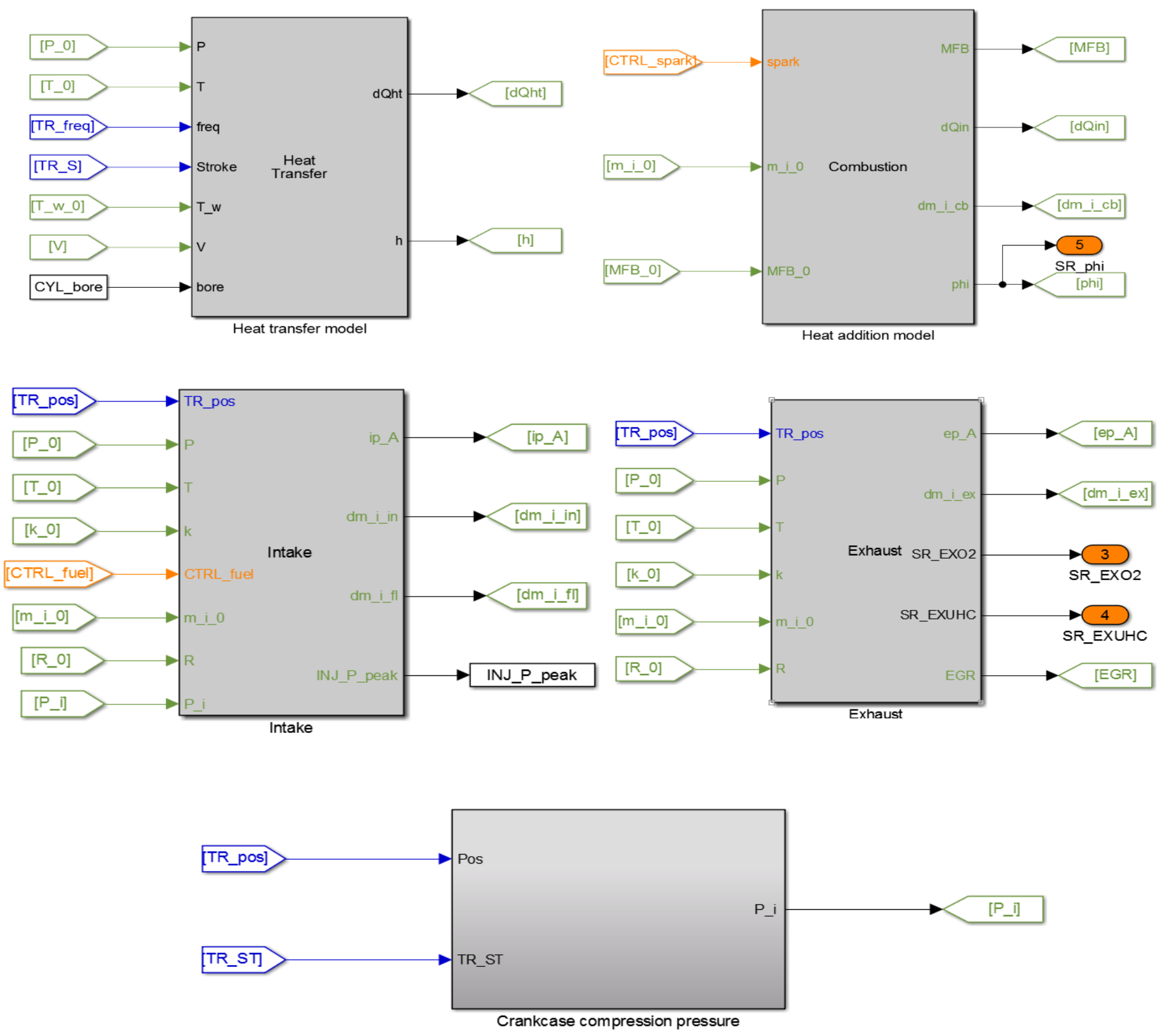

Figure 29: Multiple subsystems developed inside the engine cylinder model for calculation of cylinder pressure. 


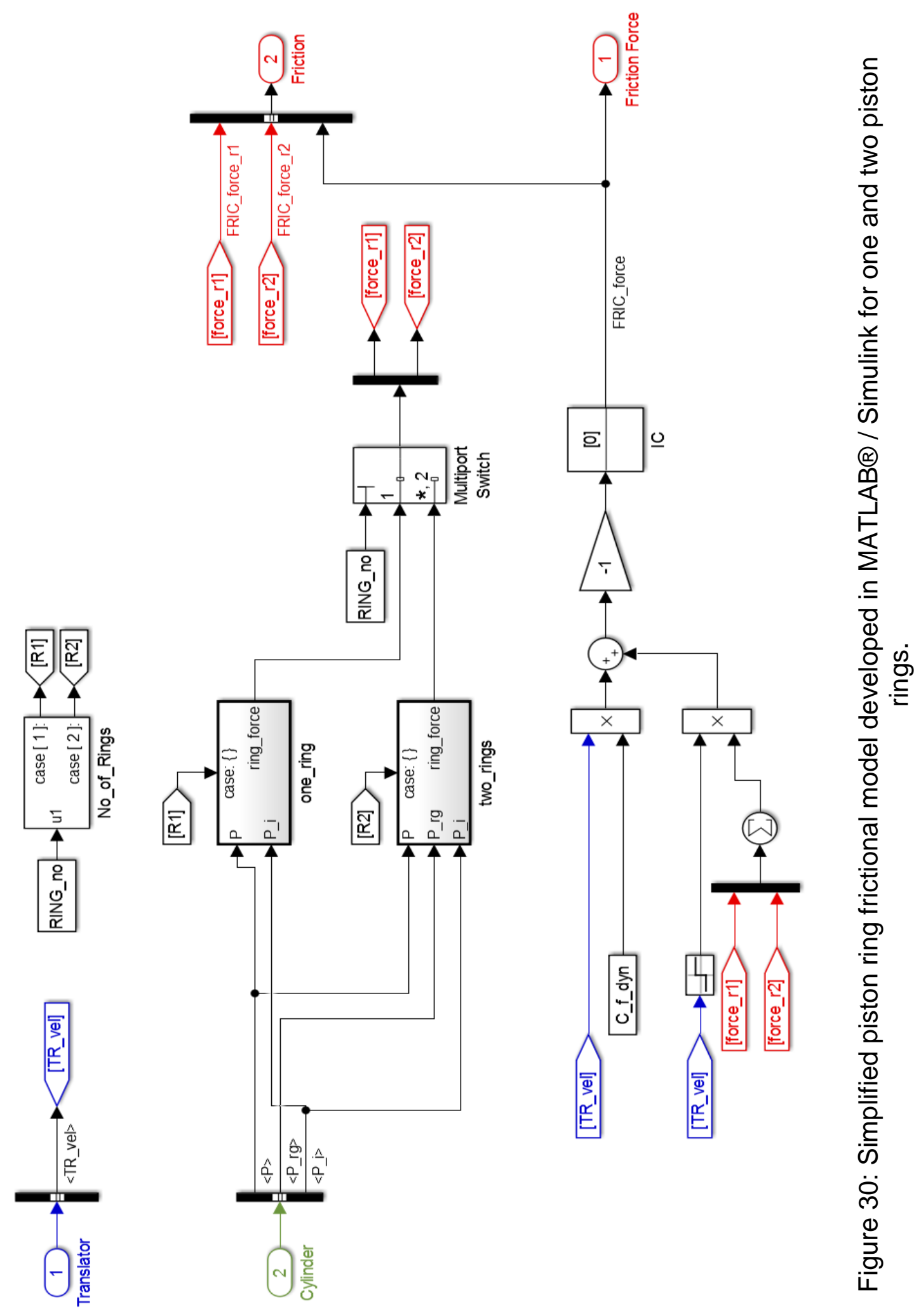




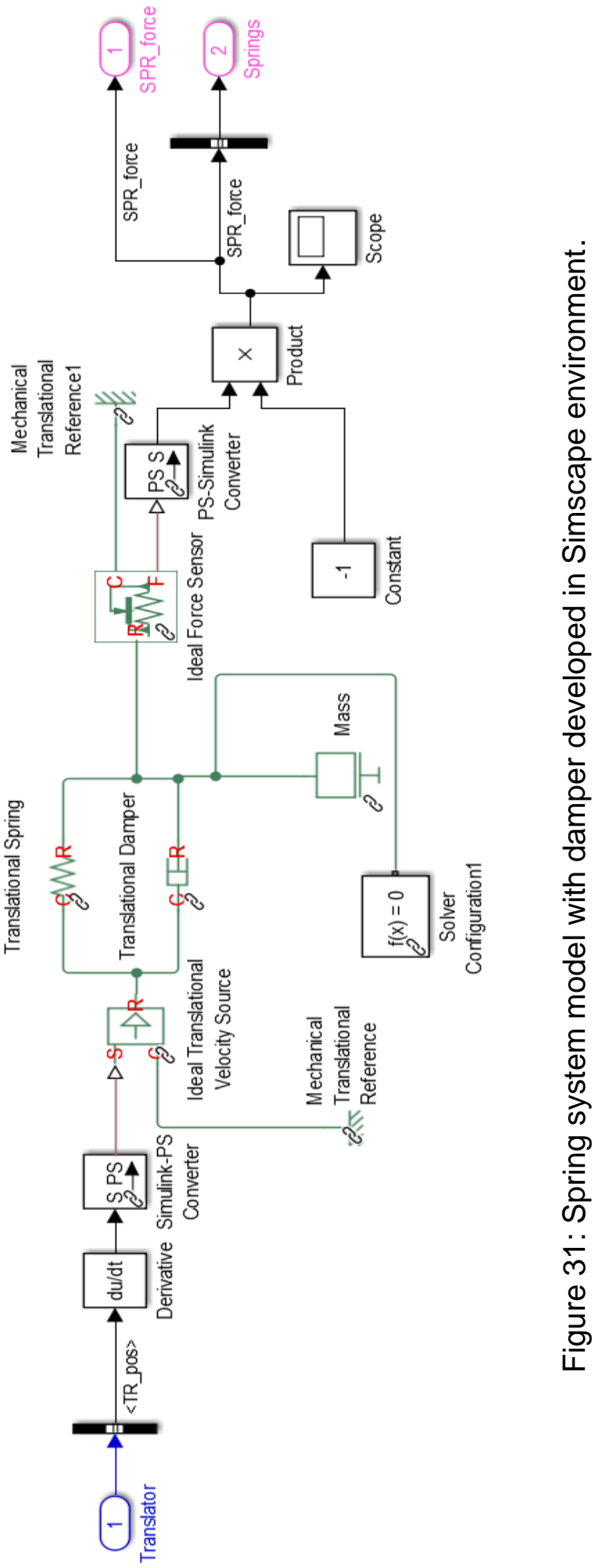


The spring stiffness and damping coefficients were obtained from the MTS testing of the experimental springs. From Figure 31, a mechanical translation reference was connected to the casing (C) port of damper and spring. This translation reference was assumed to be rigid. In reality, the experimental FPEG system mounting was not perfectly rigid due to vibrations. The damping coefficient also accounted for the vibration loss.

On a whole, the multiple subsystems presented in this section were connected logically in Simulink. The numerical model was solved by using a fixed time step approach with ordinary differential equations of multiple orders. The simulation was made to run for a total time of 10 seconds. This allowed for 800 continuous cycles of an FPEG system operating at $80 \mathrm{~Hz}$. To capture the mass flowing into and out of the cylinder domain, a time step of $5 \mu$ s was considered. Increasing the time step resulted in an unstable operation and loss of accuracy. Initially, the numerical model calculated the model parameters based on the initial conditions provided to the numerical model. This process was called as initialization. After the initialization, the rate of differentiation and mathematical equations calculated the model parameters in the next time step. This process was repeated until the end of the simulation time.

\subsection{Fundamental modeling results}

This section provided the fundamental simulation results obtained from running the single and dual cylinder FPEG numerical models. The numerical modeling results were then compared with the results from a CSE system of the same size. The single and dual FPEG system specifications used in the numerical model are provided in Table 2. The dual FPEG system considered two engine cylinders of the same displacement used in the single cylinder design. In other words, the total effective displacement of dual and single cylinder designs was $68 \mathrm{cc}$ and $34 \mathrm{cc}$. However, the stroke length was maintained the same for both the systems. Due to the FPEG inherent nature, the stroke length was changed with the energy stored in the system. However, the constant stroke length was obtained by implementing an external energy management controller. This energy management controller behavior was explained in Section 5.4. The FPEG system defined in Table 2 was considered as fundamental for all the modeling results presented in this dissertation. Moreover, it also facilitated in improving the various submodels within the numerical model. 
As mentioned in Table 2, an effective stroke of $30 \mathrm{~mm}$ was considered. This value was selected based on the experimental CSE system of the same size developed by Darzi [117]. The term effective stroke length referred to the actual translator displacement of an FPEG system. This term was different from the term maximum stroke length. The term maximum stroke length referred to the summation of an actual stroke length and clearance stroke length as shown in Figure 18. In this case, the neutral position or initial starting position was set at $15 \mathrm{~mm}$ from TDC. The dead centers, port opening, and closing locations were maintained the same for both FPEG and CSE systems. Similar engine geometric specifications were also considered the same for both the systems under comparison.

Table 2: System specifications of the single and dual cylinder FPEG systems.

\begin{tabular}{|c|c|}
\hline Engine cylinder & Specifications \\
\hline Cylinder bore & $38 \mathrm{~mm}$ \\
\hline Effective stroke & $30 \mathrm{~mm}$ \\
\hline Effective displacement & $34 \mathrm{cc}$ \\
\hline Initial position & $15 \mathrm{~mm}$ from TDC \\
\hline Wiebe form factors & 5,2 \\
\hline Fuel-air equivalence ratio & 1.0 \\
\hline Combustion efficiency & $95 \%$ \\
\hline Combustion duration & $2.25 \mathrm{~ms}$ \\
\hline Exhaust port opening & $18 \mathrm{~mm}$ from TDC \\
\hline Scavenging port opening & $24 \mathrm{~mm} \mathrm{from} \mathrm{TDC}$ \\
\hline Trapped compression ratio & 8.25 \\
\hline No of piston rings & 1 \\
\hline Moving mass & $2.75 \mathrm{~kg}$ \\
\hline LEM $\quad$ & $75 \%$ \\
\hline LEM efficiency & $75 \mathrm{~Hz}$ \\
\hline Mechanical springs & 20 \\
\hline No of springs & $558 \mathrm{kN} / \mathrm{m}$ \\
\hline Total spring stiffness
\end{tabular}

The combustion efficiency of $95 \%$ and a combustion duration of $2.25 \mathrm{~ms}$ were considered. Experimental combustion efficiency was not available at the current moment for the FPEG system. As a result, a model sensitivity study was considered in Chapter 4 to understand the effects of combustion efficiency. Moreover, an assumption of a fixed combustion period might 
yield different results than if it was defined by the piston position. This was because of the differences in the operating frequency and dynamics of both FPEG and CSE systems. However, defining the combustion duration as a function of piston dynamics was out of scope of this investigation. Moreover, a trapped compression ratio of 8.25 was selected for both the systems. The trapped compression in two-stroke engines referred to the calculation of compression ratio after the exhaust port closure.

Apart from these, a LEM efficiency of $75 \%$ was considered during the generating phase. This value included the LEM, spring, and vibration losses during the stable FPEG generation phase. This value was selected based upon the experimental FPEG system conversion efficiency. The conversion efficiency was calculated by using indicated and LEM electric power outputs. Besides the LEM efficiency, the moving mass was also assumed the same for both single and dual cylinder FPEG systems. In general, the moving mass also changes with respect to the electrical power output. This was due to the change in the LEM magnet mass for different power outputs if the same design was used for both the systems. However, the moving mass can be maintained the same by changing the LEM magnet size, design, and material properties through the optimization studies. This LEM optimization study was beyond the scope of this dissertation. The modeling results obtained with these specifications are presented in Figures 32 to 42.

Figure 32 shows the variation in the translator velocity plotted against the translator displacement. The dynamics were plotted for both single and dual FPEG systems along with the CSE system of the same effective displacement and operating frequency. The position of ' $0 \mathrm{~mm}$ ' referred to the initial point or neutral point location. The positions of $-15 \mathrm{~mm}$ and $+15 \mathrm{~mm}$ referred to TDC and BDC locations respectively. In a dual cylinder FPEG system, the TDC and BDC locations represented the actual TDC and BDC of a left engine cylinder. For the right engine cylinder, these positions represented the actual BDC and TDC. The terms 'EPO/EPC' referred to the exhaust port opening and closing locations. The terms 'SPO/SPC' referred to the scavenging port opening and closing locations. The port opening and closing locations were represented by red dotted lines in Figure 32. For a detailed understanding of system dynamics, the entire cycle was divided into three regions. Region 1 referred to the operation of the system when the ports were closed. Region 2 considered the region after the opening of an exhaust port and before the opening of scavenging ports. Region 3 considered the region after the opening of scavenging ports during which the exhaust port was also open. 


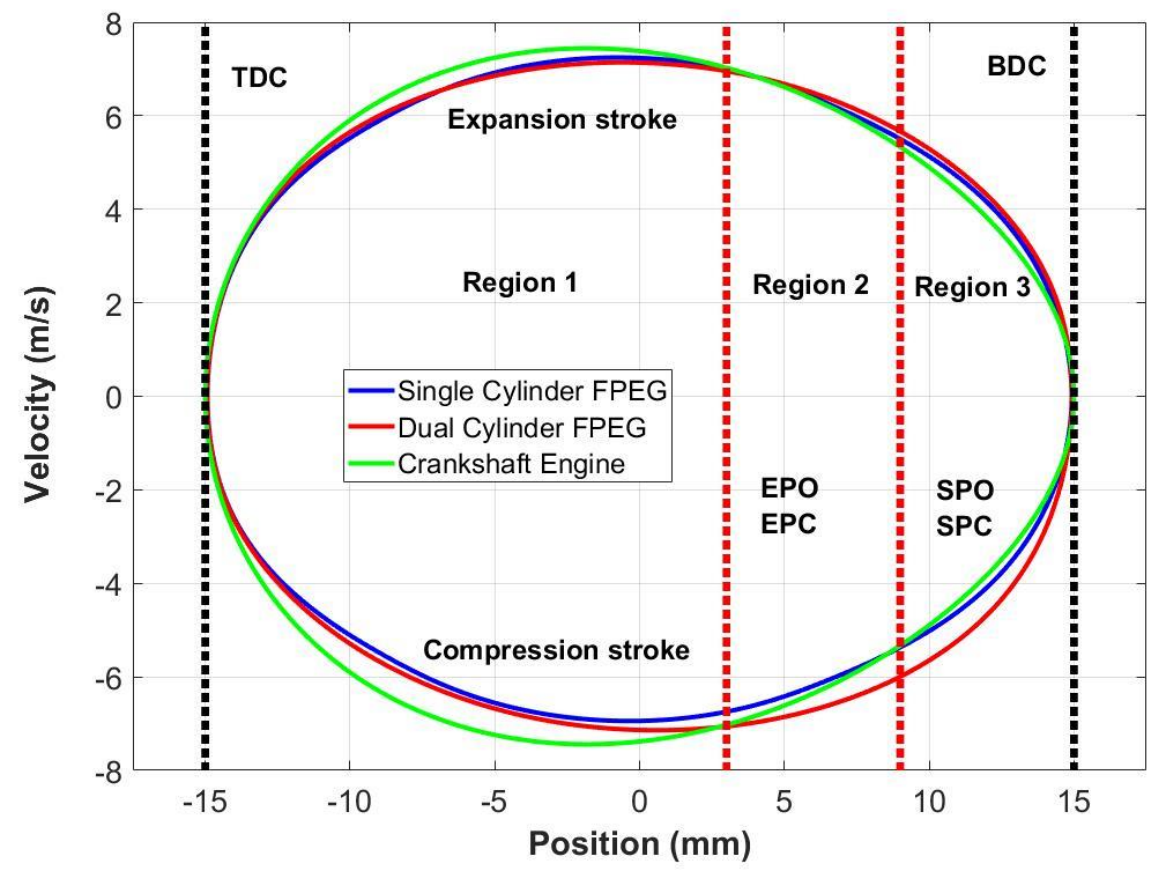

Figure 32: Velocity versus position plot of single FPEG, dual FPEG and CSE systems.

The translator dynamics plot shown in Figure 32 was different from the FPEG dynamics without springs [18]. With the addition of stiff springs, the velocity and position distribution were almost similar to a sinusoidal distribution. Moreover, this spring dominant system had a slightly slower expansion stroke than the non-spring dominant system. The expansion stroke was almost similar to a CSE system as seen in Region 1. In Region 1, during the expansion stroke, the single and dual cylinder FPEG systems operated at a lower velocity than the CSE system for the same cycle frequency. This was due to the inherent nature of stiff springs and the geometry of a crankshaft and connecting rod mechanism. The same distribution was seen during the compression stroke in Region 1. The major differences in the dynamics were observed in Regions 2 and 3. The Regions 2 and 3 were together called as a gas exchange region. In Regions 2 and 3 , during the expansion stroke, the CSE operated at a lower velocity than the FPEG systems. As a result, the CSE possessed more time to blow down the exhaust products before the SPO. Moreover, it spent more time near BDC when compared to the FPEG systems. Owing to the less time spent at BDC, the scavenging efficiency was poor for the FPEG systems for the same intake and exhaust conditions. However, the amount of fuel slip was also reduced for the FPEG systems when 
operated on the same exhaust pressure conditions. This was because of the variations in the time spent by the piston in Regions 2 and 3.

In Regions 2 and 3, during the compression stroke, the dual cylinder FPEG system exhibited a higher piston velocity than the rest of the systems. This was due to the presence of additional combustion forces from the right engine cylinder in a dual FPEG system. Moreover, the dual FPEG system frequency $(80.32 \mathrm{~Hz})$ was $4.5 \mathrm{~Hz}$ higher than the single FPEG system frequency $(75.86 \mathrm{~Hz})$ due to these combustion forces. For the comparison study, a CSE of $80 \mathrm{~Hz}$ was considered. The variation in the acceleration with respect to velocity for these three systems is shown in Figure 33.

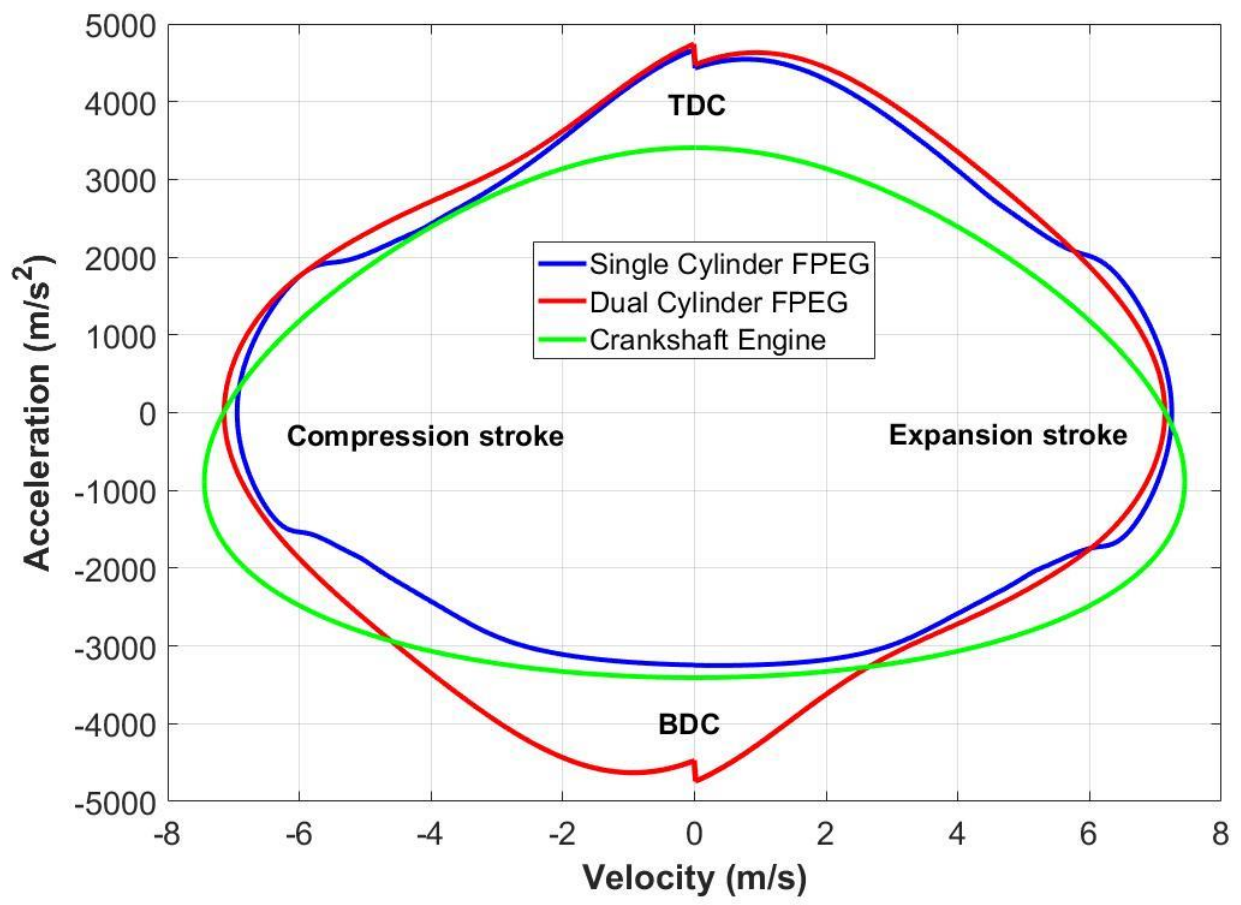

Figure 33: Translator acceleration and velocity distribution for CSE and FPEG systems.

The deviations in the dynamics were more noticeable in Figure 33 than in Figure 32. From Figure 33, the FPEG systems operated at a higher acceleration near TDC than the CSE system. This high acceleration reduced the time spent by the FPEG system near TDC. As a result, the high cylinder temperature was maintained only for a short time when compared to the CSE system. A 
sudden drop in the acceleration was seen at TDC and BDC for FPEG systems. This was mainly because of the reversal of frictional force at the dead centers. Moreover, the frictional forces defined the dynamics in the FPEG systems. Since CSE motion was defined by a crankshaft and connecting rod mechanism, this sudden peak was not observed in a CSE system. Moreover, the slight acceleration change was also maintained by the flywheel connection in a CSE system.

The variation in the pressure and volume (PV) plot for the CSE and FPEG systems is shown in Figure 34. Similar to the velocity and position plot, the PV diagram was also divided into three regions. Moreover, the PV diagram of the CSE system was obtained from the experiments. When compared to the CSE system, the FPEG systems achieved a higher peak cylinder pressure. This high peak pressure was mainly because of a lower translator velocity of FPEG systems than the CSE. As the piston velocity was high, the volume change between any two consecutive time steps was higher for the CSE system than the FPEG systems. This high volume chance reduced the peak pressure in the CSE than the FPEG systems.

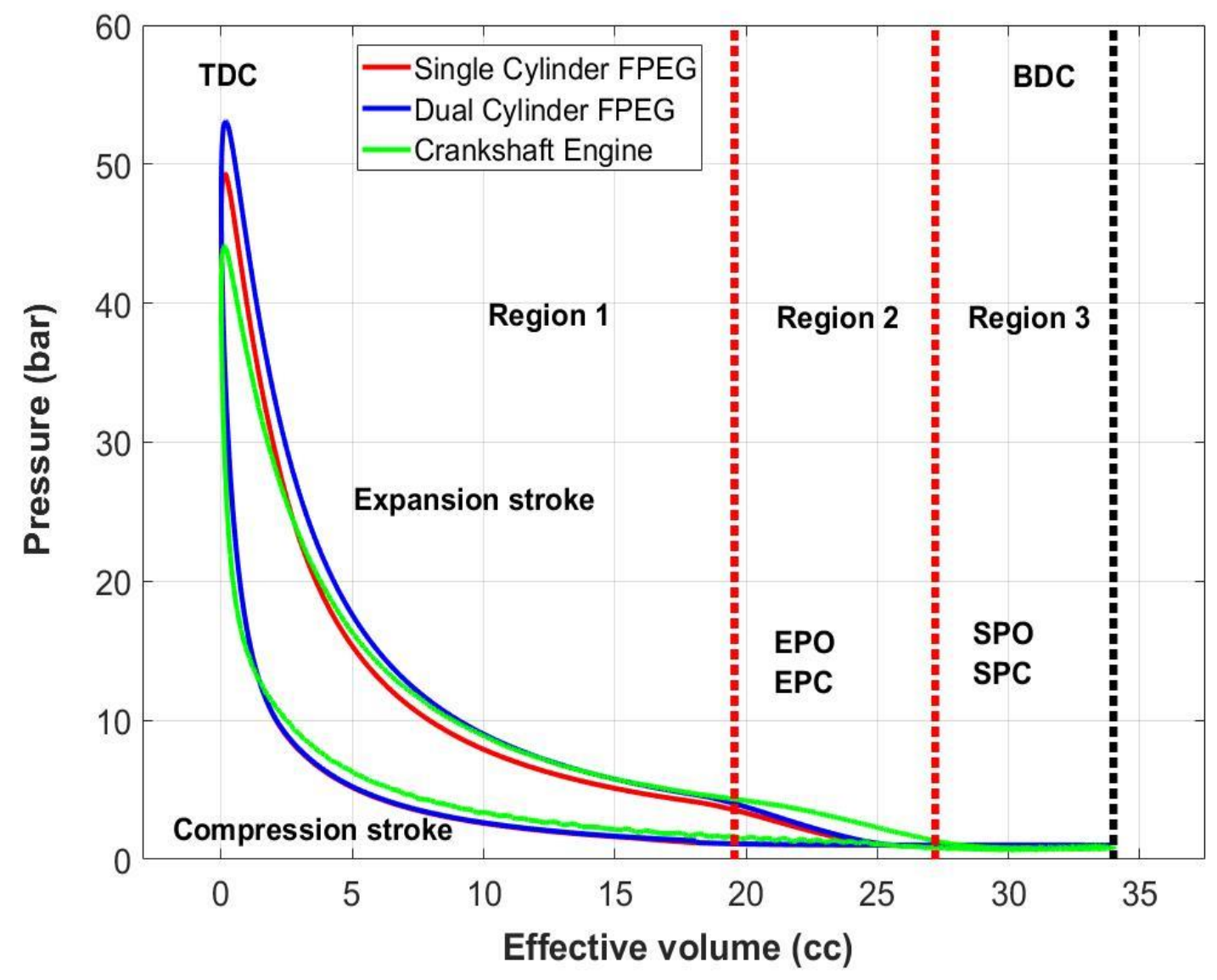

Figure 34: PV diagrams of CSE and FPEG systems. 
The zoomed portion of the PV diagram's Region 2 is shown in Figure 35. The oscillations in the CSE pressure was due to the experimental sensor pressure measurements. From Figure 35, the cylinder pressure of a CSE in Region 2 was higher when compared to the FPEG systems. One of the reasons for that behavior was because of the dynamics variations in CSE and FPEG systems. As the piston velocity was low, the rate of change in the volume was also low. This reduced volume change was responsible for a higher cylinder pressure in a CSE system than the FPEG systems. In addition to the dynamics, the presence of a resonant exhaust system in the CSE was also responsible for a high cylinder pressure. The zoomed portion of the PV diagram's Region 3 is shown in Figure 36. The FPEG systems followed the same path for both the compression and expansion processes. This was because of modeling the intake mixture flow at a constant intake pressure in the numerical model. The CSE used a resonant intake system. As a result, the PV diagram of a CSE system followed different paths for expansion and compression strokes. Moreover, the CSE was also helpful in flowing more amount of fuel and air mixture in the combustion chamber. This was because of the increased pressure gradient between the intake and the cylinder pressure. This pressure gradient guided the flow of fuel and air mixture into the engine cylinder.

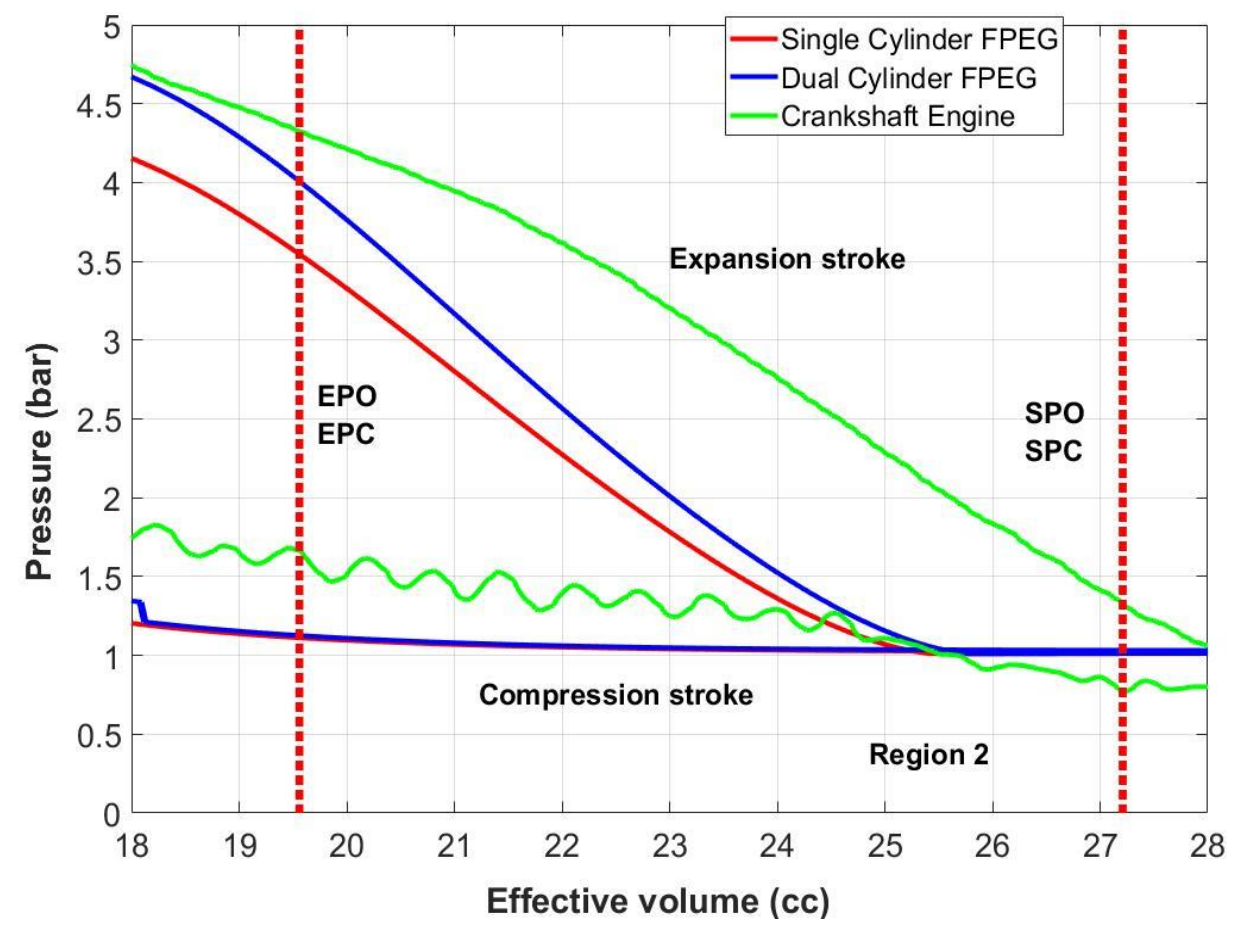

Figure 35: Region 2 of PV diagram for CSE and FPEG systems. 


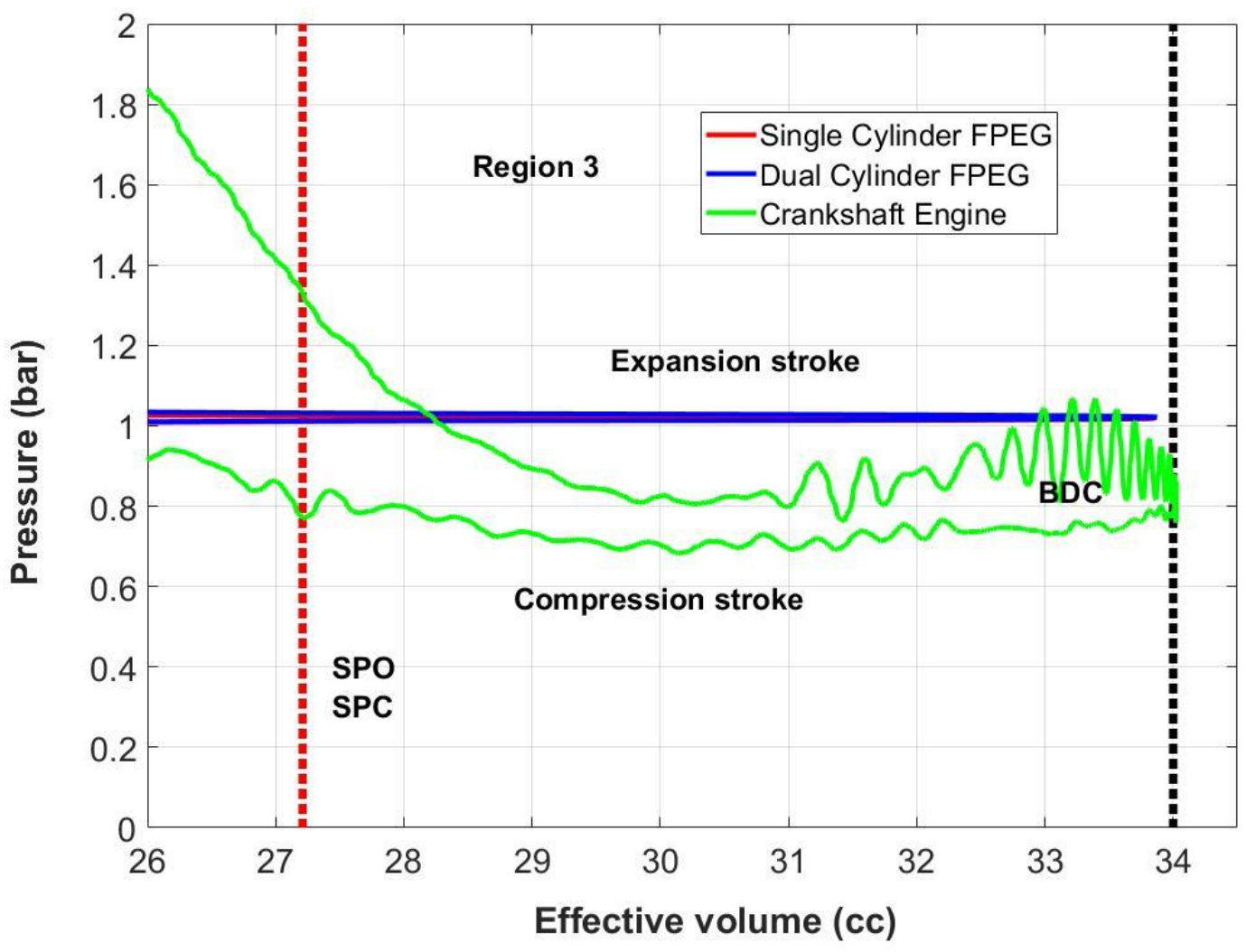

Figure 36: Region 3 of PV diagram for crankshaft and FPEG systems.

In a dual cylinder FPEG system, the same conditions were maintained for both left and right cylinders. The PV diagram was identical for both the cylinders as seen in Figure 37. Based on the dominance of forces acting on the translator rod, the FPEG systems were classified into three types. The first type referred to a spring dominant FPEG system. This system was considered in this dissertation. In a spring dominant FPEG system, the spring forces acting on the translator rod were higher than the forces generated by the combustion of fuel. As spring forces dominated the combustion forces, the springs were mainly responsible for the translator dynamics. The nuances in the combustion process had a minimal impact on translator dynamics. The main advantage of such a spring dominant system was a consistent and sinusoidal motion. Moreover, the cycle-tocycle variations were also reduced when compared to a non-spring dominant system. However, there were also some disadvantages associated with the spring dominant system. The spring and vibration losses were the main drawbacks of the spring dominant systems. 


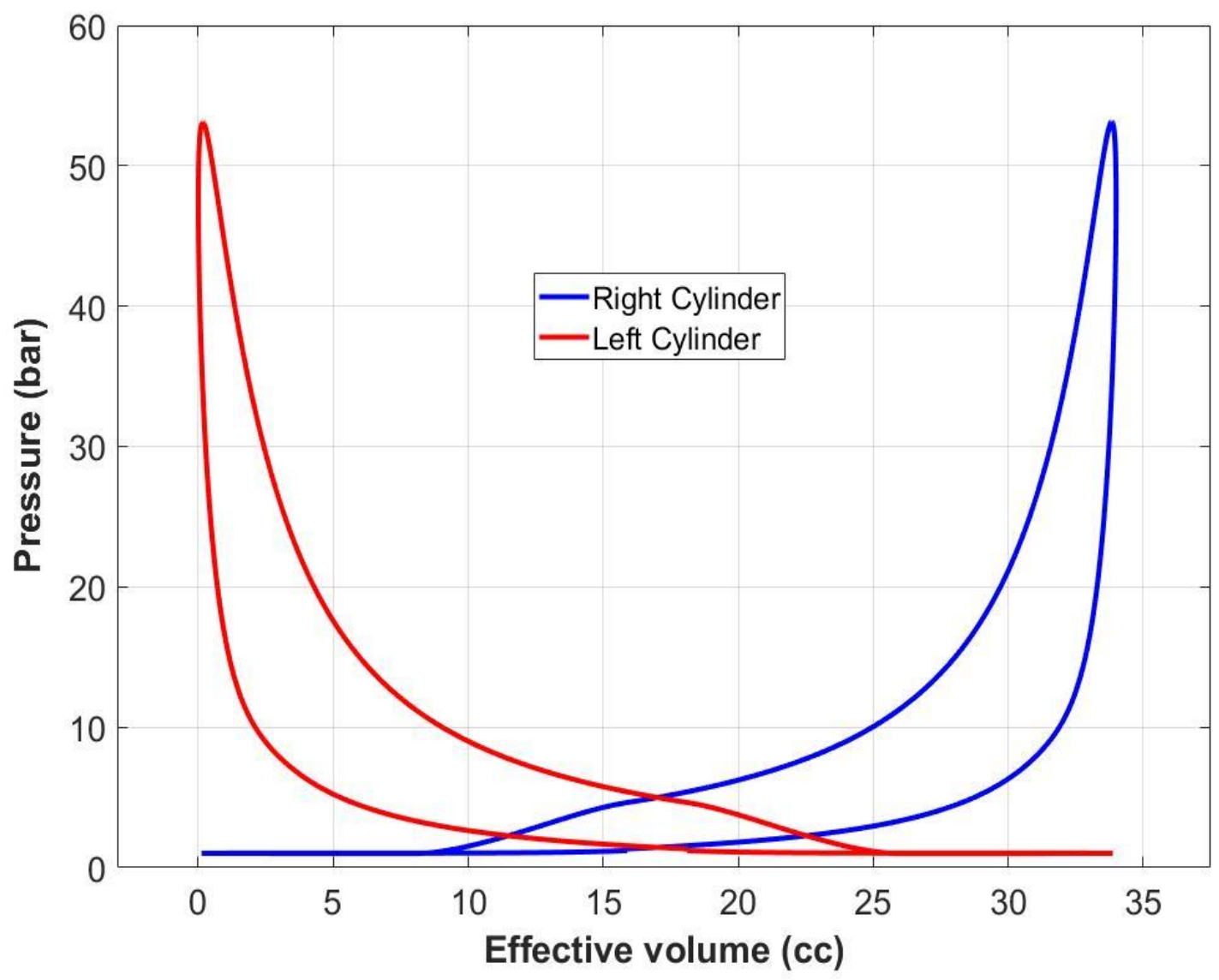

Figure 37: PV diagram of left and right engine cylinders in a dual FPEG system for an individual effective volume of $34 \mathrm{cc}$.

The second type of classification referred to an equally dominant system. In an equally dominant FPEG system, the peak combustion and peak spring forces were of the same magnitude. The system behavior was in between the spring dominant system and the FPEG system without springs. In this system, both spring and combustion forces impacted the translator dynamics substantially. The third classification referred to a combustion driven FPEG system. This was also called as a no-spring FPEG system. This system needed two cylinders for its operation. While one cylinder acted as an engine cylinder, another cylinder acted as a spring/energy storage device. In this system, the spring forces were absent or negligible. In this system, the combustion forces defined the translator dynamics and operating frequency. The advantages of this system were the faster expansion stroke and reduced heat transfer losses when compared to the spring dominant system. However, the cycle-to-cycle variations were also high due to variations in the 
combustion process. In other words, a robust control methodology was required to maintain the stable combustion process in this system.

The distribution of forces in single and dual cylinder FPEG systems is shown in Figure 38. The distribution started and ended at BDC. For a single cylinder FPEG system, a single cycle of operation was considered. For a dual cylinder FPEG system, two cycles were considered. This was to show the distribution of combustion forces from both the engine cylinders.
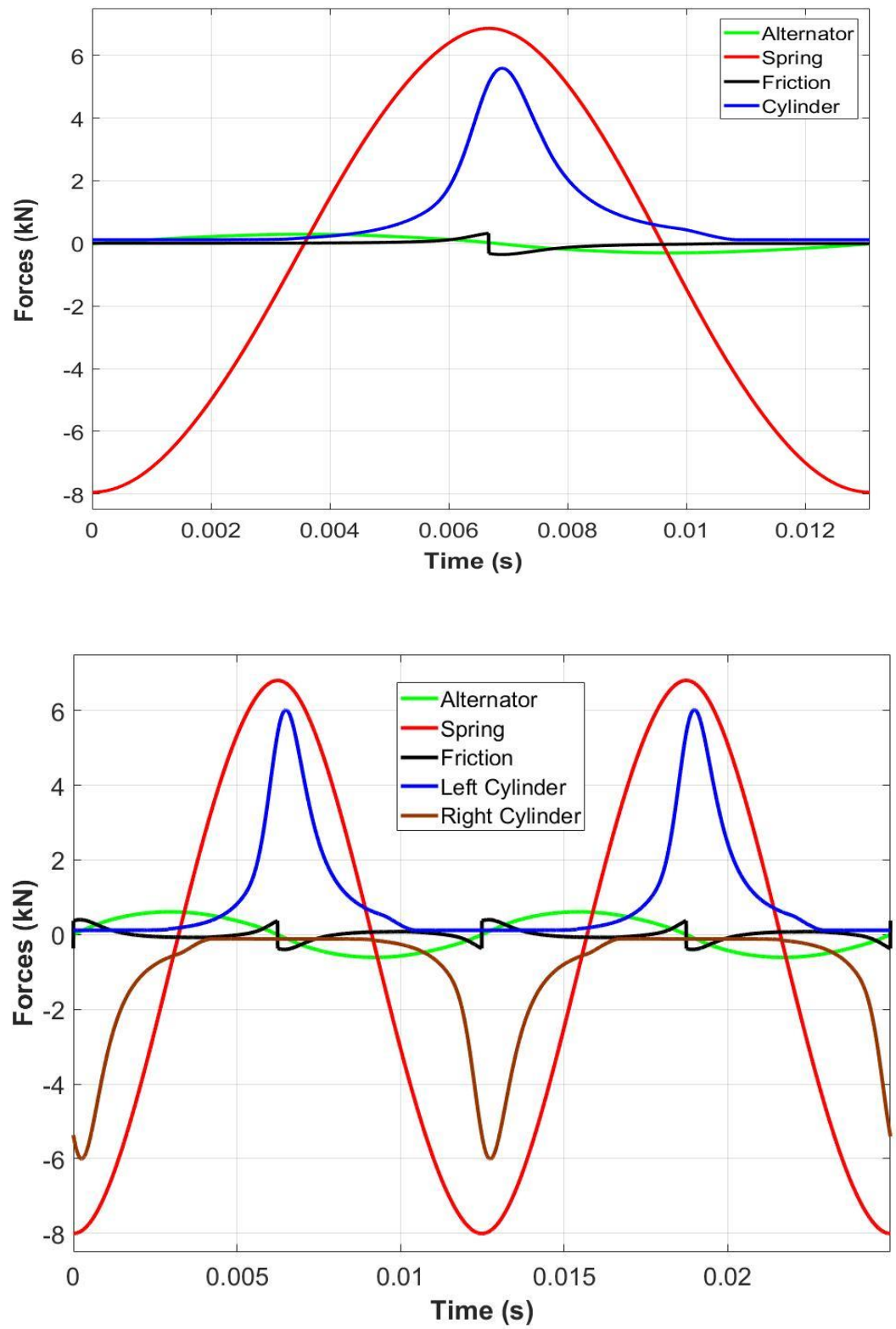

Figure 38: Distribution of forces in single and dual FPEG systems. 
Based on Figure 38, the peak spring forces were $25 \%$ higher than the peak combustion forces. Because of this dominance, the FPEG system was concluded as a spring dominated FPEG system. Moreover, the frictional forces were high in the dual cylinder FPEG system at BDC. This was due to the presence of friction from the second engine cylinder.

The distribution of instantaneous electrical power for single and dual cylinder FPEG systems is shown in Figure 39. The solid lines represented an instantaneous power output. The dotted lines represented the mean electrical power. From Figure 39, the dual FPEG system achieved a higher power output than the single cylinder system for the same displacement of $34 \mathrm{cc}$. This was because of the presence of additional combustion energy in the dual FPEG system. Moreover, the shift in the power distribution was mainly because of the operating frequency.

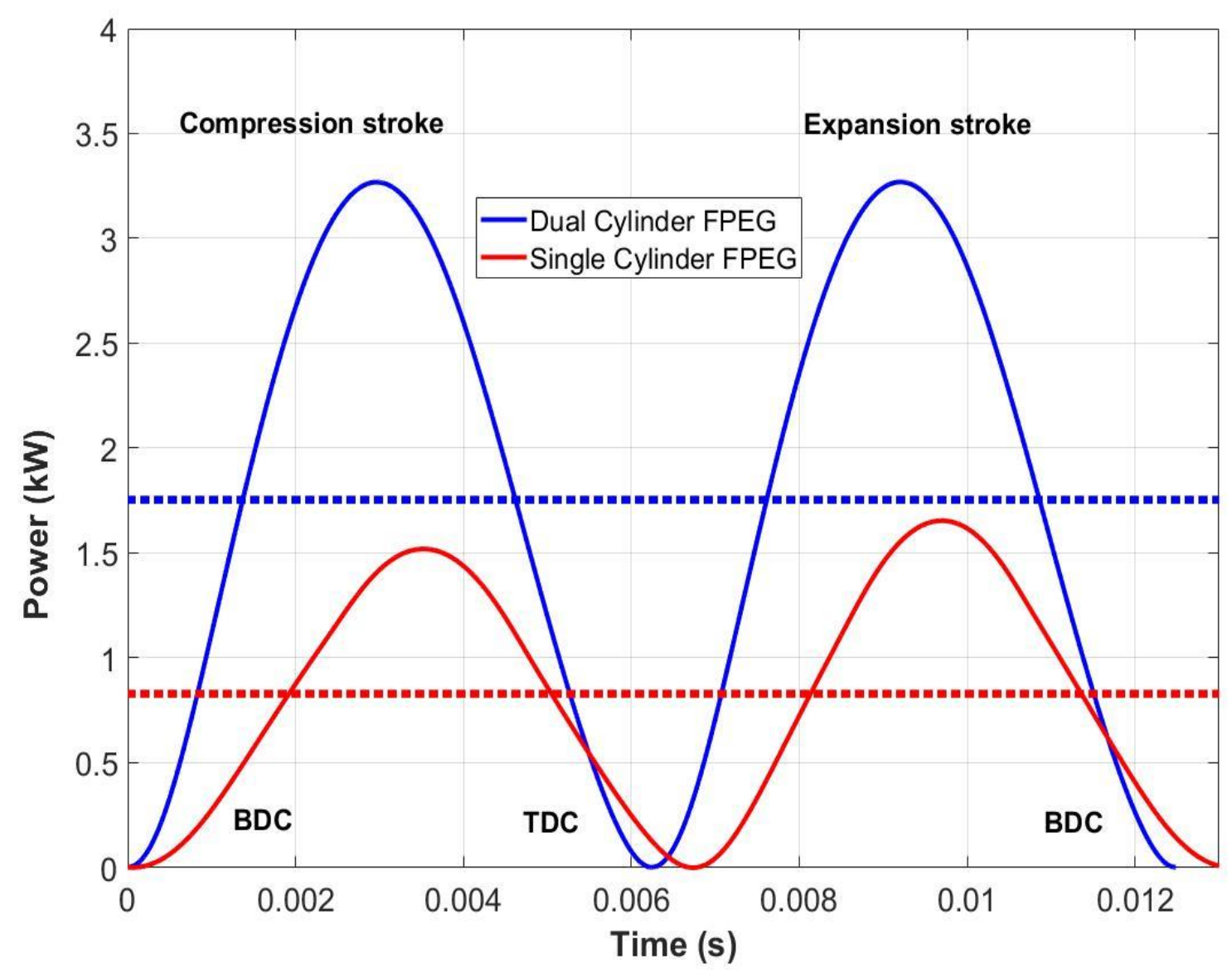

Figure 39: Instantaneous power distribution for single and dual FPEG systems. 
The operating frequency of a dual FPEG was $4.5 \mathrm{~Hz}$ higher than the single FPEG system. However, the dual cylinder FPEG system obtained the same power peaks. This was because of the symmetry in the velocity profile around the neutral point in this system. The symmetry was obtained due to the presence of combustion forces on both sides in a dual cylinder FPEG system. In a single cylinder FPEG system, the expansion stroke power peak was $13.3 \%$ higher than the compression stroke power peak. This high power peak in the expansion stroke was because of the combustion forces.

In terms of mean electric power, the single and dual FPEG systems generated $827.5 \mathrm{~W}$ and 1752.7 W respectively. The mean electric power of a dual FPEG system was $110 \%$ higher than the mean electric power of a single FPEG system. For the same operating conditions and fuel input, the dual cylinder should provide twice the electrical power than the single cylinder output. Figure 40 shows the time available for the gas exchange process of FPEG and CSE systems operating under the same conditions.

The term free blow down process in Figure 40 referred to the time period between the EPO and SPO. The term forced scavenging referred to the time period between the SPO and SPC. During this time, the exhaust port was also open. The term additional exhaust process referred to the time period between the SPC and EPC. The total gas exchange duration was the summation of the free blow down, forced scavenging and additional exhaust duration [118]. The variations in the dynamics and combustion characteristics of these engines caused the differences in the durations in the gas exchange process. From Figure 40, the total gas exchange duration of a dual cylinder FPEG system was the lowest. This was because of the presence of combustion forces from another engine cylinder at BDC. These additional combustion forces caused an increase in the translator velocity at BDC. Of all the systems, the CSE had the highest gas exchange duration of $6.05 \mathrm{~ms}$. This was due to the inherent motion defined by the crankshaft and connecting rod mechanism. When compared to the CSE, the total gas exchange duration was reduced by $12.1 \%$ and $2.5 \%$ for dual and single FPEG systems. Similarly, the scavenging duration alone was reduced by $14.8 \%$ and $5.7 \%$ for dual and single cylinder FPEG systems respectively. 


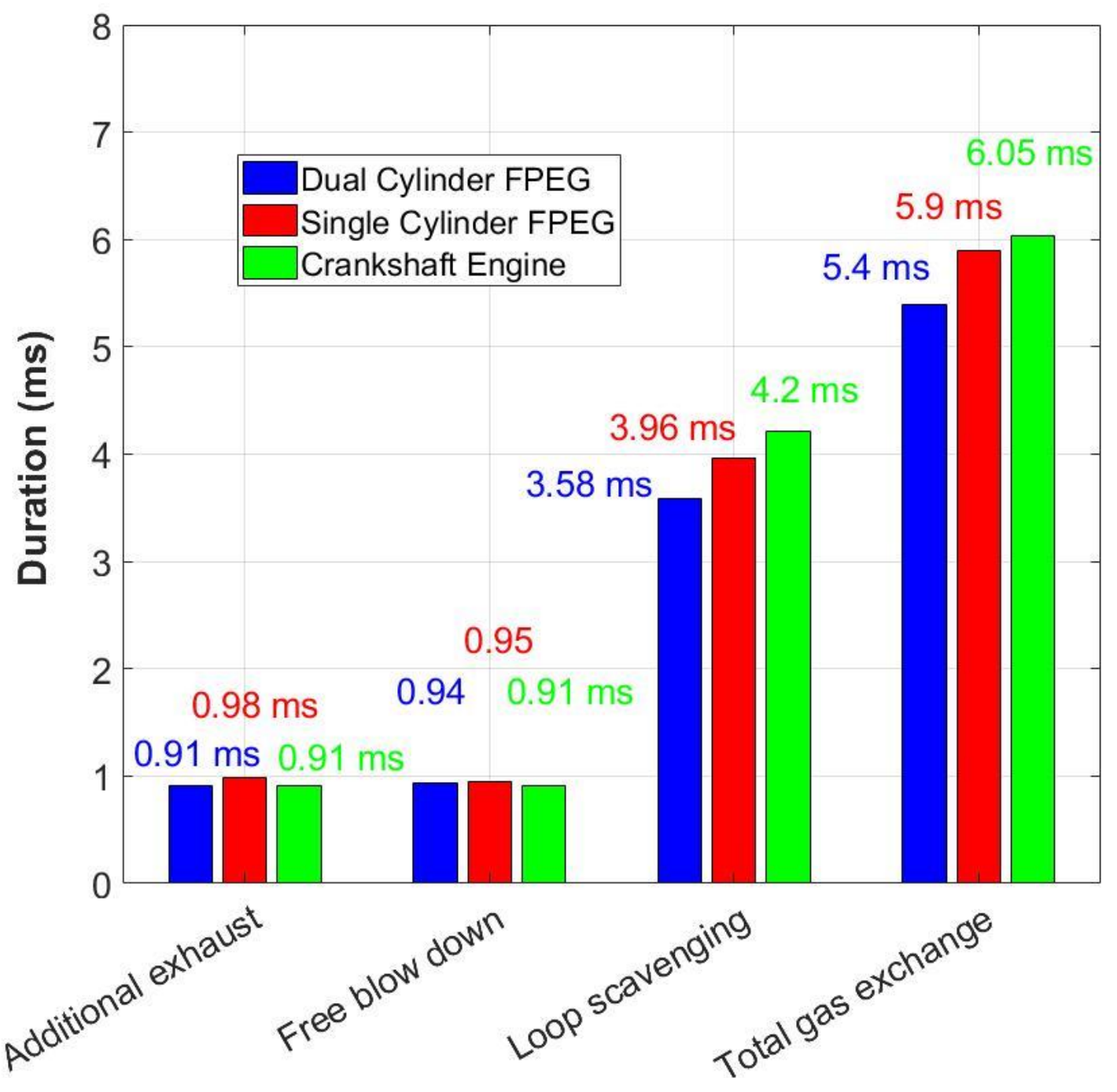

Figure 40: Duration of gas exchange process for FPEG and crankshaft systems.

\subsection{Energy analysis using the first law of thermodynamics}

In this investigation, the first law of thermodynamics was used to quantify the energy distribution for the FPEG systems. The first law was implemented at the modeling level by using a MATLAB® script. The model callback command in the Simulink executed this MATLAB® script after the completion of the simulation. The total fuel energy in the FPEG system was mainly divided into seven sinks [119]. 
1. FPEG system efficiency

2. LEM losses

3. Frictional losses

4. Exhaust heat energy losses

5. Fuel slip

6. Heat transfer losses

7. Combustion losses

Initially, the amount of fuel energy (' $\dot{E}_{f u e l}$ ') supplied per second was calculated. The fuel energy supply also included the fuel lost in the short-circuiting. The corresponding mathematical equation is given in Eq. 33.

$$
\dot{E}_{f u e l}=\dot{m}_{f u e l} * L H V
$$

Eq. 33

After calculating the fuel energy rate, the engine indicated efficiency (' $\eta_{\text {ind }}$ ') and FPEG system efficiencies (' $\eta_{F P E G}$ ') were calculated as follows.

$$
\begin{gathered}
\eta_{\text {ind }}=\frac{W_{\text {indicated }} * f}{\dot{E}_{\text {fuel }}} \\
\eta_{F P E G}=\frac{P_{\text {electrical }}}{\dot{E}_{\text {fuel }}}
\end{gathered}
$$

Eq. 34

The terms ' $W_{\text {indicated }}$ ' and ' $P_{\text {electrical }}$ ' represent the indicated work and electrical power output respectively. The difference in the indicated to the FPEG system efficiency was accounted for friction, LEM, spring and vibrational losses. In this dissertation, the frictional losses were calculated from the frictional forces modeled in the system. Other than the friction losses, the spring, vibration and LEM machine losses were categorized under one category as LEM losses. The corresponding equations for frictional and LEM losses are provided below.

$$
\eta_{f r i c}=\frac{F_{f r i c} * \dot{x}}{\dot{E}_{f u e l}}
$$




$$
\eta_{L E M}=\eta_{\text {ind }}-\eta_{F P E G}-\eta_{\text {fric }}
$$

Eq. 35

Moreover, the modeling also accounted for the mass balance of all the species in the combustion chamber. The molar mass fraction of all species was calculated at every time step. Based on the mass management model, the exhaust heat energy and fuel slip loss were calculated. The corresponding equations for exhaust heat energy and fuel slip losses are given in Eq. 36.

$$
\begin{gathered}
\eta_{\text {fuel slip }}=\frac{\dot{m}_{\text {fuel out }} * L H V_{\text {fuel }}}{\dot{E}_{\text {fuel }}} \\
\eta_{\text {exhaust heat }}=\frac{\dot{m}_{\text {out }} c_{p}\left(T_{\text {out }}-T_{\text {ref }}\right)}{\dot{E}_{\text {fuel }}}
\end{gathered}
$$

Eq. 36

The heat transfer in this investigation was calculated by using the Hohenberg heat transfer correlation. The heat transfer losses were obtained from the heat transfer rate. Finally, the remaining of the fuel energy losses was accounted for combustion losses.

The distribution of the fuel energy for single and dual cylinder FPEG systems is shown in Figure 41. Based on the calculations, the single and dual cylinder FPEG system efficiencies were found to be $16.8 \%$ and $17.8 \%$. The dual cylinder system showed a $1 \%$ improvement in system efficiency. This was because of reduced fuel slip losses, increased indicated work and operating frequency. Moreover, this slight increase in the FPEG efficiency was also attributed to the modeling assumptions. The fuel slip accounted for $33.5 \%$ and $24.8 \%$ in single and dual cylinder FPEG systems for the same operating conditions. The reduction in the fuel slip losses was mainly attributed to the reduced scavenging losses in a dual cylinder system. In other words, the fuel slip was reduced by $8.7 \%$ in a dual cylinder FPEG system. However, the LEM, heat transfer and frictional losses together were increased by $7.8 \%$. This was due to an increase in the cylinder temperature, electrical power output, translator velocity, and operating frequencies. The comparison of indicated, system, and conversion losses for CSE and FPEG systems is presented in Figure 42. 

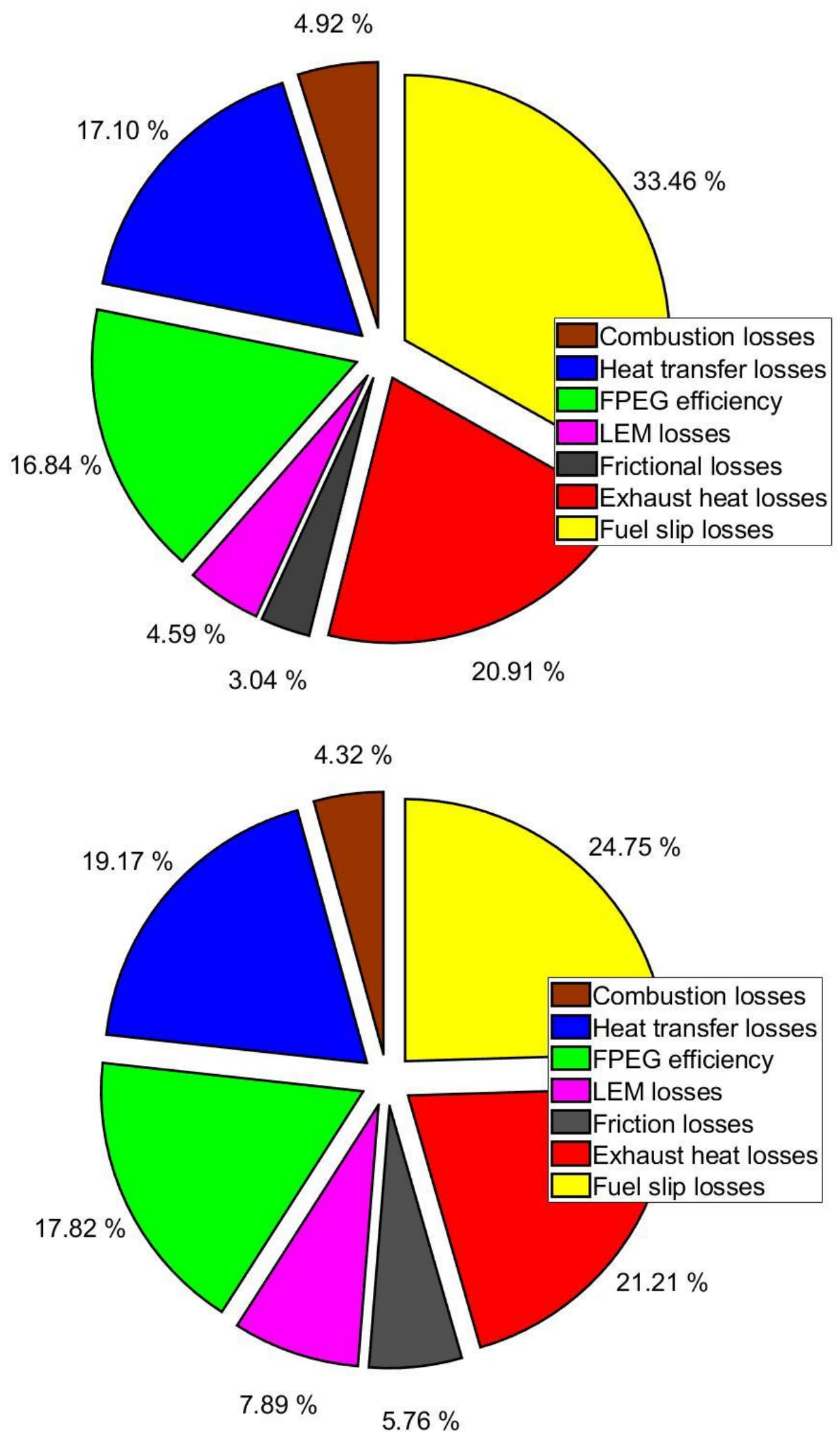

Figure 41: Energy distribution of single (top) and dual (bottom) FPEG systems. 
From Figure 42, of all the systems, the dual cylinder FPEG system produced the highest indicated efficiency of $31.56 \%$. This was due to unique FPEG dynamics and reduced fuel slip losses. The overall system efficiency in Figure 42 referred to the electrical and brake efficiencies in FPEG and CSE systems. The overall system efficiency was calculated by using electrical and brake power. Based on these calculations, the single, dual, and CSE systems achieved a system efficiency of $17.84 \%, 16.84 \%$, and $19.59 \%$. Even though the indicated efficiencies were higher for the spring dominant FPEG systems, the CSE achieved a higher system efficiency than the FPEG systems.

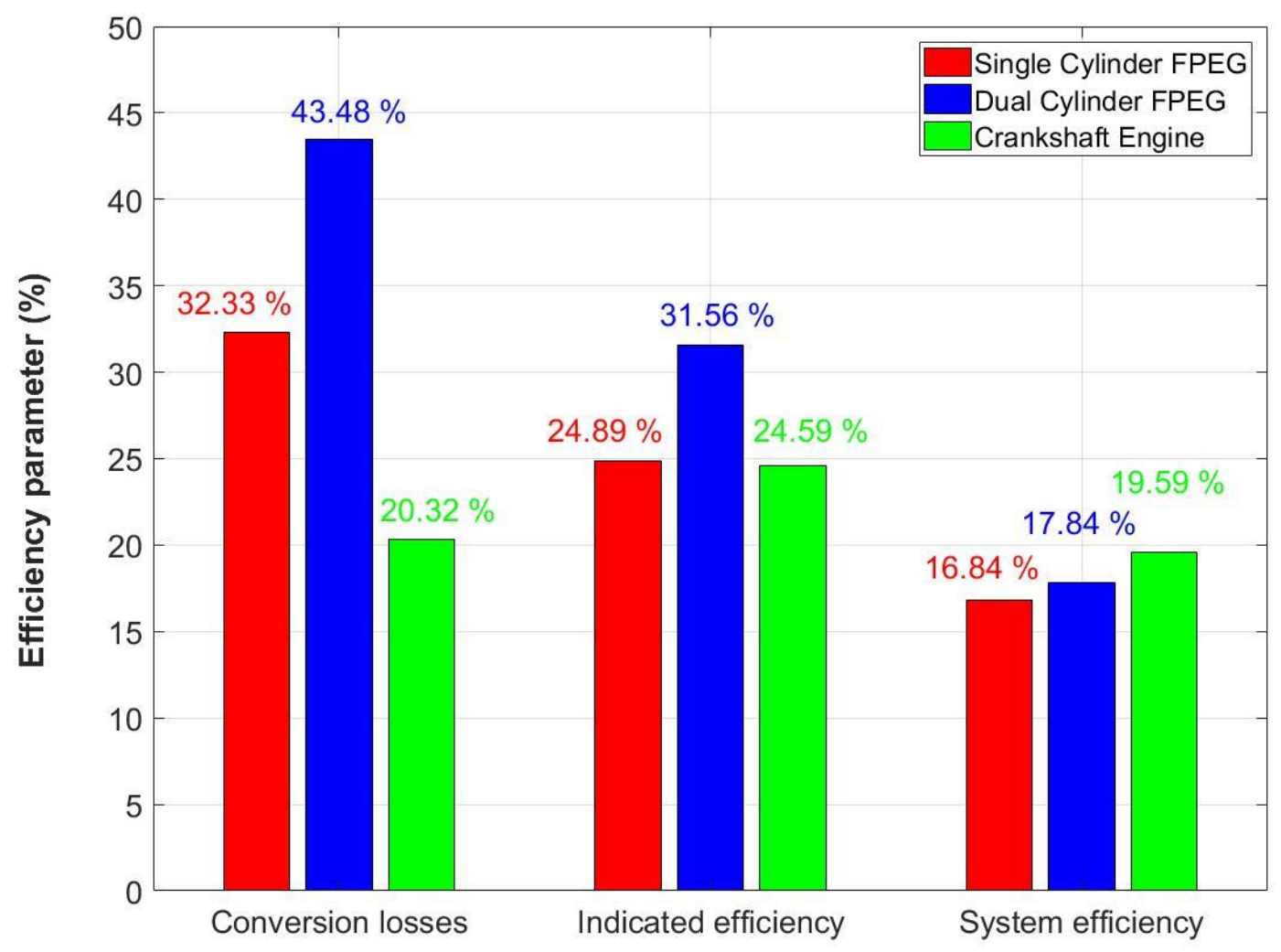

Figure 42: Comparison of indicated, system and conversion losses for FPEG and CSE systems.

From Figure 42, the conversion inefficiency of CSE was found to be $20.32 \%$. For dual and single cylinder FPEG systems, it was found to be $32.33 \%$ and $43.48 \%$. This was due to the presence of additional conversion losses in the spring dominant FPEG system. In a CSE, the conversion losses accounted for piston ring and crankshaft connecting rod bearing friction. In the FPEG systems, the conversion losses also included losses from vibration, stiff springs, and LEM machine losses along with the CSE conversion losses. 


\subsection{Effect of spring stiffness}

A parametric investigation of spring stiffness was performed for both single and dual cylinder FPEG systems. The moving mass of the translator was divided into two components. The two components were the static and dynamic mass. The static mass included the mass of piston assembly, LEM moving magnets, and translator rod. The dynamic mass considered the moving mass of the springs. This dynamic mass was changed linearly with the variation in spring stiffness. Moreover, the ignition timing was fixed at an optimized ignition timing for all the cases. The optimized ignition timing was calculated using an algorithm defined in Section 5.5. Apart from the spring stiffness and translator mass, all other parameters were held constant. The spring stiffness parametric sweep results provided interesting insights on FPEG system dynamics and performance.

The variation in the dual and single cylinder dynamics for different spring stiffness is shown in Figure 43. The black arrows represented the direction of increasing spring stiffness. Increasing the spring stiffness increased the maximum velocity in both the configurations. Furthermore, the velocity profile shape became more symmetric and sinusoidal with increasing spring stiffness. This symmetry was measured about the neutral or initial position. In a dual cylinder FPEG, a linear trend in the maximum velocity was observed with increasing spring stiffness. However, the maximum velocity was non-linear at a low spring stiffness in a single cylinder configuration. This was due to high cycle-to-cycle variations. These variations were caused by the dominance of combustion forces over the spring forces.

The variations in the cylinder pressure with spring stiffness for dual and single cylinder FPEG systems are shown in Figure 44. Three trends were observed in the pressure versus displacement diagram with varying spring stiffness. Firstly, increasing the spring stiffness decreased the maximum cylinder pressure in both the configurations. This reduction in the cylinder pressure peak also reduced the indicated work with increasing spring stiffness. Secondly, the amount of exhaust gas retained in the cylinder increased with increasing stiffness. This was due to maintaining the same intake and exhaust pressures for all spring stiffness studies. At the lowest spring stiffness, the cylinder pressure in Region 2 reduced much faster when compared to the highest spring stiffness. This was due to the dynamics variation and change in the pressure gradient with different spring stiffness. Moreover, the blow down of the burned products was 
affected because of the pressure gradient. The pressure gradient and the amount of exhaust gas retained were decreased and increased respectively with increasing the spring stiffness.
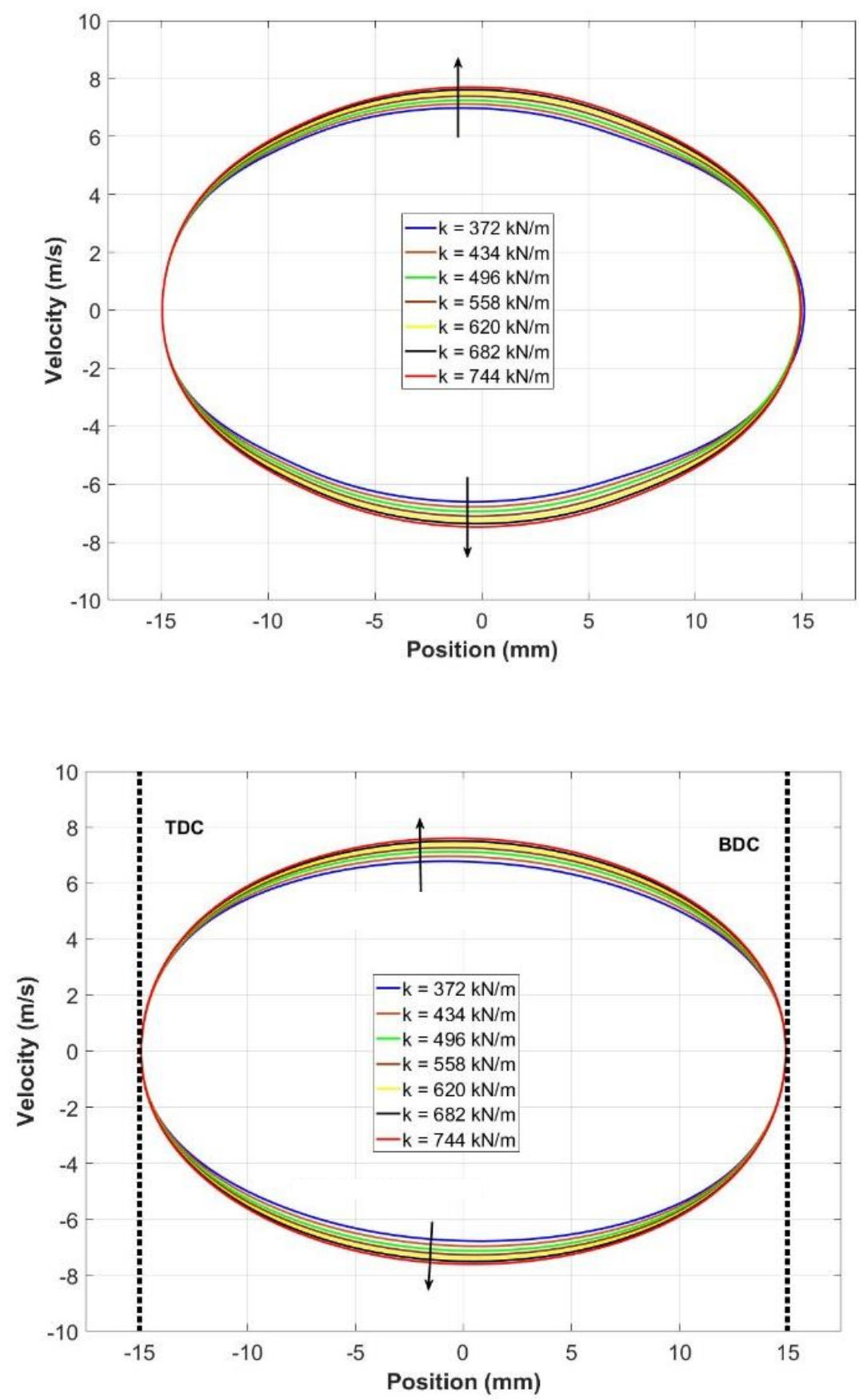

Figure 43: Variation in translator velocity with increasing spring systems (arrows) in single (top) and dual (bottom) cylinder FPEG systems. 

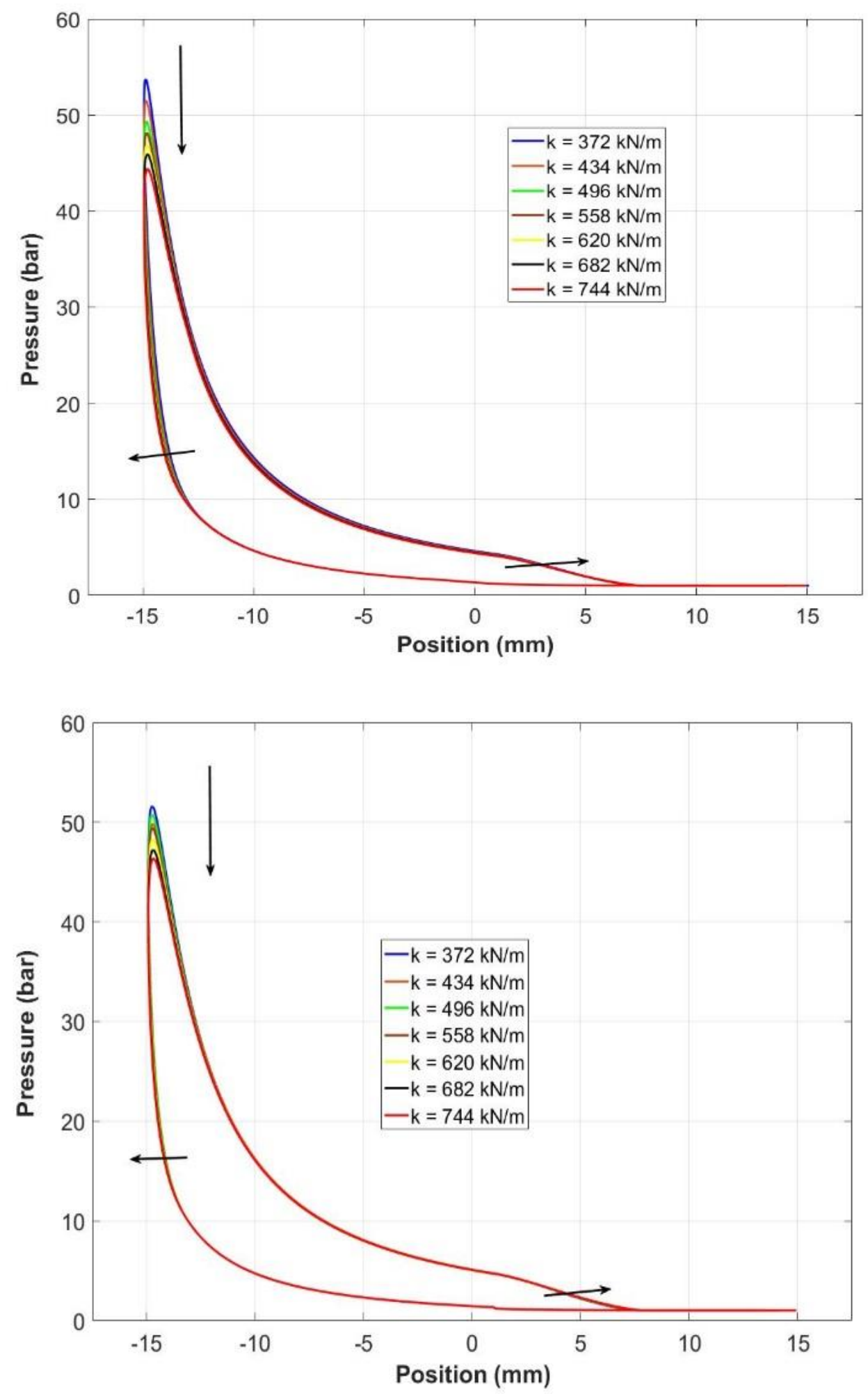

Figure 44: Variation in cylinder pressure with increasing spring systems (arrows) in single (top) and dual (bottom) cylinder FPEG systems. 
For the spring stiffness studies, the FPEG systems were made to run at a stoichiometric fuel air mixture ratio. As the exhaust gas retained increased with increasing spring stiffness, the amount of fuel delivered into the system was reduced. With the reduction in fuel mass, the input fuel energy was also reduced. As a result, the cylinder pressures were reduced due to reduction in the fuel input.

Thirdly, the small cumulative changes in the cylinder volume increased the peak cylinder pressure at the lowest spring stiffness. In other words, with increasing spring stiffness, the difference in the cylinder volume between any two consecutive time steps was increased. As the volume was increased, the cylinder pressure was decreased with increasing spring stiffness. Based on the results, the spring stiffness variation can be used for the optimization of the combustion process in the FPEG systems.

The variations in the frequency and electrical power output with spring stiffness are shown in Figure 45 . The slightly erratic nature of results in Figure 45 suggested the numerical model dependence. In other words, the response of various submodels was responsible for the erratic nature of results. In Figure 45, the parametric investigation results were normalized with the results obtained from the fundamental case. With increasing spring stiffness, the frequency was increased from $76 \mathrm{~Hz}$ to $84 \mathrm{~Hz}$ in a dual cylinder configuration. In a single cylinder configuration, it was increased from $72.3 \mathrm{~Hz}$ to $80.8 \mathrm{~Hz}$. This corresponds to the $10.5 \%$ and $11.8 \%$ increase in frequency with increasing spring stiffness from $372 \mathrm{kN} / \mathrm{m}$ to $744 \mathrm{kN} / \mathrm{m}$.

Similarly, the electrical power output was increased by $200 \mathrm{~W}$ in a dual cylinder FPEG system. In a single cylinder design, this value was increased by $80 \mathrm{~W}$. This corresponds to the $8 \%$ increase in the electric power with increasing the spring stiffness. In both the cases, the LEM open-circuit voltage was maintained the same with the same moving mass. The main reason for the increase in the indicated power was mainly due to the dominant effect of frequency. For example, in a single cylinder version, the frequency was increased by $10.6 \%$ with increasing spring stiffness. However, the indicated work was reduced by $2.3 \%$ with increasing the spring stiffness. In the numerical model, the indicated power was calculated by multiplying the indicated work and operating frequency. Based on the values, the indicated power was increased by $8 \%$. As a result, the dominant effect of the frequency over the indicated work increased the indicated power with increasing spring stiffness. 

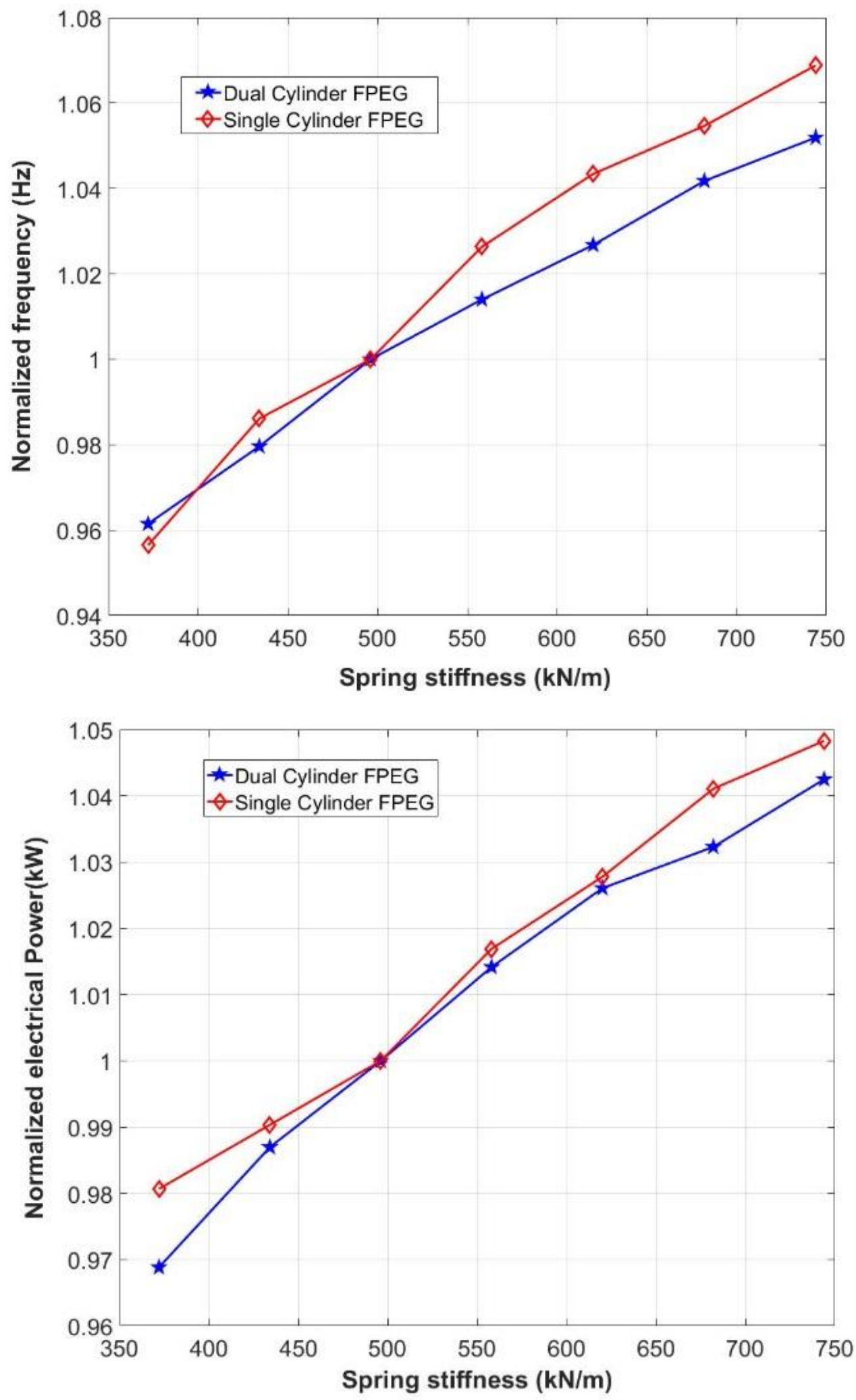

Figure 45: Normalized frequency and electrical power trend with increasing spring stiffness. 


\subsection{Piston ring frictional model results}

As mentioned in Section 2.2.6, an iterative based frictional model along with the coupling methodology was developed in MATLAB®. This model was used to simulate the frictional characteristics of a piston ring. After numerous iterative calculations, the numerical procedure was converged and completed. Initially, the fundamental case dynamics and combustion characteristics were provided to the frictional model. The variations in the piston dynamics and combustion characteristics caused the variations in the piston ring frictional characteristics.

The variations in the minimum oil film thickness for both FPEG and CSE systems are shown in Figure 46. The results were plotted against crankshaft degrees for easier understanding. The equivalent crank angle in an FPEG system was calculated by multiplying the instantaneous cycle time with frequency. The simulation started and ended at BDC for all the systems. In general, three different variations were observed in the oil film distribution presented in Figure 46.

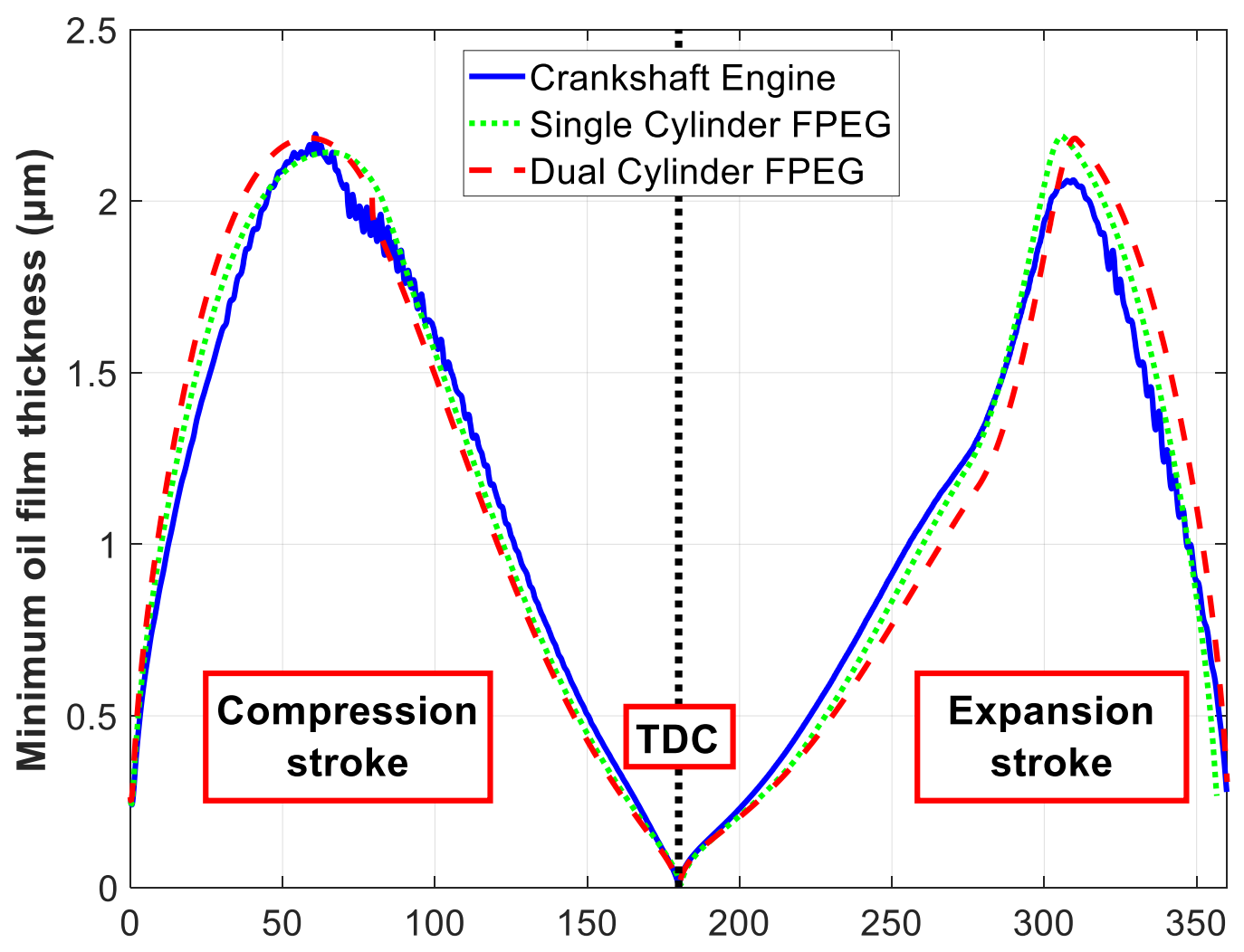

Figure 46: Comparison of minimum oil film thickness distribution for FPEG and CSE systems. 
At first, a thicker oil film was observed for FPEG systems at the beginning of the compression stroke. This thick oil film was caused by the sufficient hydrodynamic pressures. The hydrodynamic pressure was mainly dependent upon sliding velocity, operating conditions, and applied load. In this region, the higher FPEG translator velocity than the CSE system increased the hydrodynamic pressure. Considering only the FPEG systems, the dual FPEG system attained a thick oil film than a single FPEG system. This was again due to the variation in the sliding velocities near BDC. In other words, the FPEG systems had a superior performance over the CSE system in this region. The second stage considered the ending and beginning stages of compression and expansion strokes respectively. In the second stage, the oil film thickness was thin for the FPEG systems. This thin oil film in the FPEG systems was mainly because of three reasons. The reasons were the reduced sliding velocities, increased in-cylinder pressures, and reduced oil viscosities.

The third stage considered the ending stage of the expansion stroke. In this stage, especially after the EPO, a thick oil film was observed in the FPEG systems. This was again because of the higher FPEG sliding velocities than the CSE system near BDC. Overall, only minor variations were observed in the minimum oil film distribution for these systems. Moreover, the addition of stiff springs was also responsible for the minor variations in the minimum oil film thickness. With the presence of stiff springs, the single cylinder FPEG dynamics were identical to the CSE dynamics with minimal deviations. Moreover, two similar oil film peaks were observed for both CSE and FPEG systems. This was due to stable translator dynamics. In a CSE, this stable movement was achieved by the crankshaft and connecting rod mechanism. In the FPEG systems, this stable translator movement was obtained by the addition of stiff springs.

Figure 47 shows the variation in the total frictional forces for both FPEG and CSE systems. The total frictional force was calculated by using the summation of hydrodynamic and asperity frictional forces. The frictional force distribution in Figure 47 was divided into five regions. Region 1 and Region 5 were related to the full fluid lubrication regions. Region 2 and Region 4 were related to the partial or mixed lubrication regions. This mixed lubrication regime was the combination of both hydrodynamic and boundary lubrication regimes. Region 3 was related to the boundary or asperity contact region. These regions were defined based on the ratio of minimum oil film thickness and composite surface roughness. 


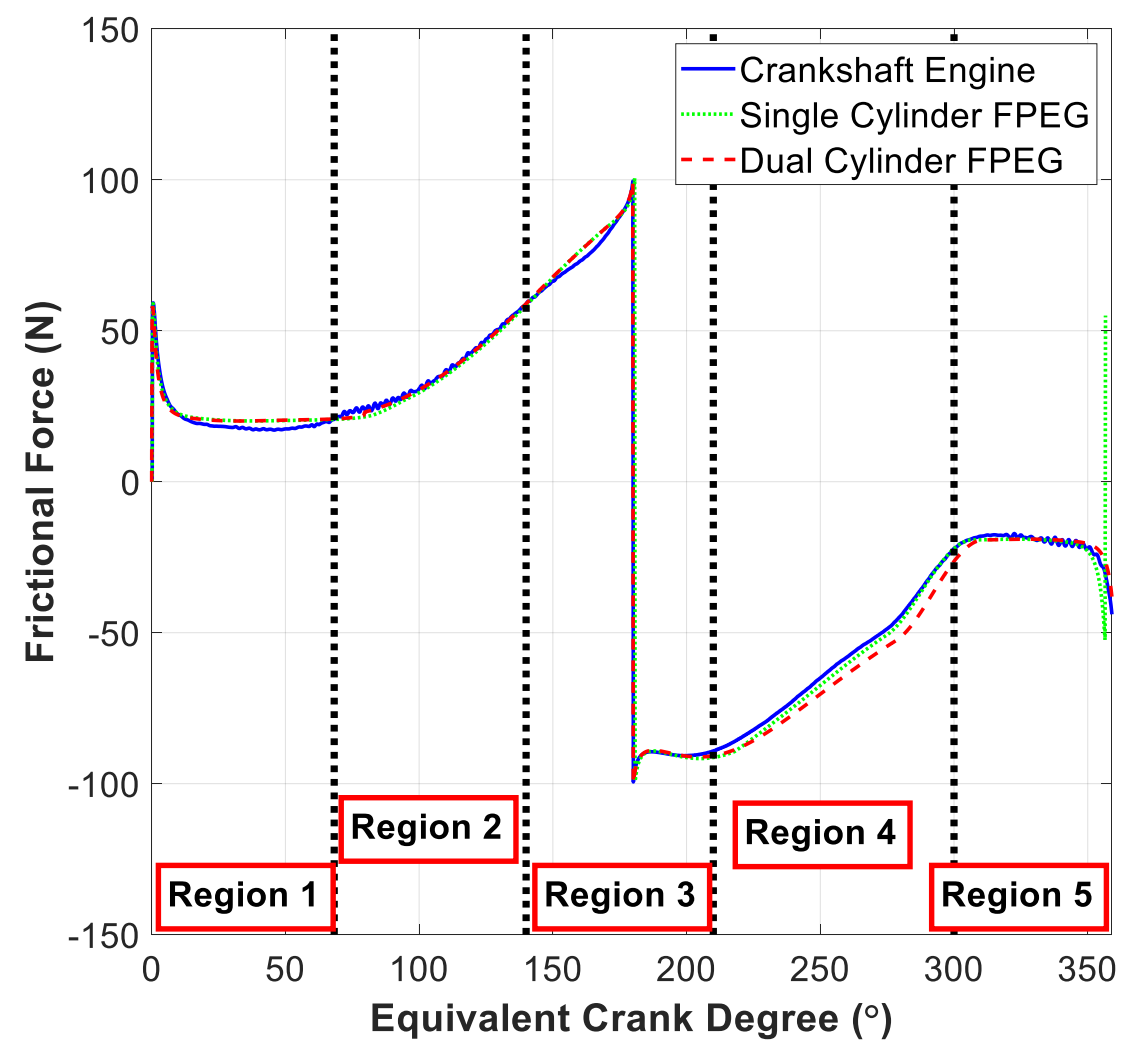

Figure 47: Comparison of piston ring frictional forces for FPEG and CSE systems.

In the full fluid lubrication regions, the single and dual FPEG systems experienced higher frictional forces than the CSE system. The friction in these regions was mainly due to the viscous action of the lubricating oil. Thicker the oil films higher the viscous losses in these regions. The FPEG systems in these regions experienced thicker oil films than the CSE system. Therefore, the FPEG frictional forces were higher than the CSE system in these regions. Moreover, the frictional losses in this regime were less when compared to the other regimes. This was due to the lower cylinder and hydrodynamic pressures in this regime when compared to the other regimes. In the mixed hydrodynamic regions, the frictional forces were mainly defined by the cylinder pressures. Due to high cylinder pressures, the FPEG systems had higher frictional forces than the CSE system. In the asperity contact region, the oil film thickness was zero. This was because of the zero translator velocity, high cylinder pressure, and low lubricant viscosity. As a result, the frictional force reached the highest value in this region. Moreover, the frictional force had both positive and negative peaks in this region. This switch in the frictional force was because of the piston reversal process at TDC. 
Figure 48 shows the variation in the total frictional power losses in CSE and FPEG systems. In the full fluid lubrication regions, the variations between in the frictional losses of FPEG and CSE systems were less. In the mixed hydrodynamic regions, the CSE system had the higher frictional power losses than the FPEG systems. This was due to a higher translator velocity of the CSE system than the FPEG systems in these regions. In the asperity contact region, the frictional losses were lower than the mixed hydrodynamic friction losses. This was due to the lower translator velocities near the TDC region when compared to the mid-stroke region. Overall, the mean frictional loss was found to be $184.7 \mathrm{~W}, 161.2 \mathrm{~W}$, and $340.8 \mathrm{~W}$ for CSE, single and dual cylinder FPEG systems. The higher friction losses in the dual cylinder than the single cylinder design was due to the presence of two engine cylinders. These two cylinders had two piston rings in total rather than one ring in the CSE and single cylinder designs. In terms of percentage, these frictional losses consumed $16.5 \%, 14.1 \%$, and $15.0 \%$ of indicated power for CSE, single and dual FPEG systems respectively.

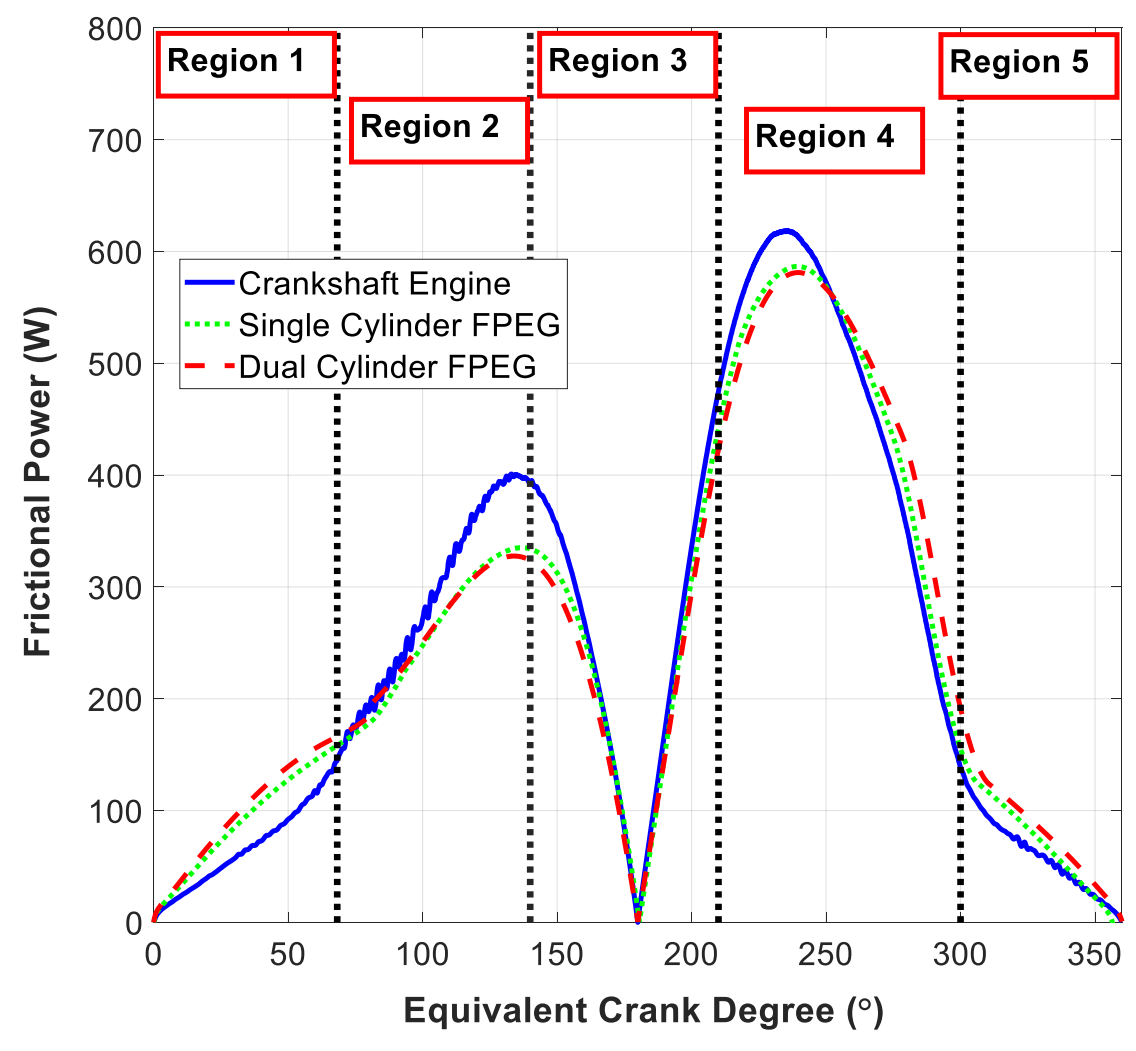

Figure 48: Distribution of instantaneous frictional power loss due to piston ring in FPEG and CSE systems. 
Similar to the fundamental case, a parametric investigation of spring stiffness was considered. This parametric investigation helped in understanding the variation of frictional characteristics with increasing spring stiffness. Moreover, the parametric investigation was considered only for a single cylinder FPEG system. This is because of the similarity in the results of single and dual FPEG systems with increasing spring stiffness. Initially, the dynamics and combustion characteristics presented in Section 2.5 were inputted into the piston ring frictional model. Figure 49 shows the variation in the minimum oil film thickness with increasing spring stiffness. The minimum oil film thickness was increased with increasing spring stiffness in all stages of compression and expansion stroke. The major factor that contributed to this increase was the translator velocity. With increasing spring stiffness, the sliding velocities were increased. Moreover, the oil viscosities and cylinder pressures were reduced with increasing spring stiffness. All these three factors together increased the minimum oil film thickness with increasing spring stiffness.

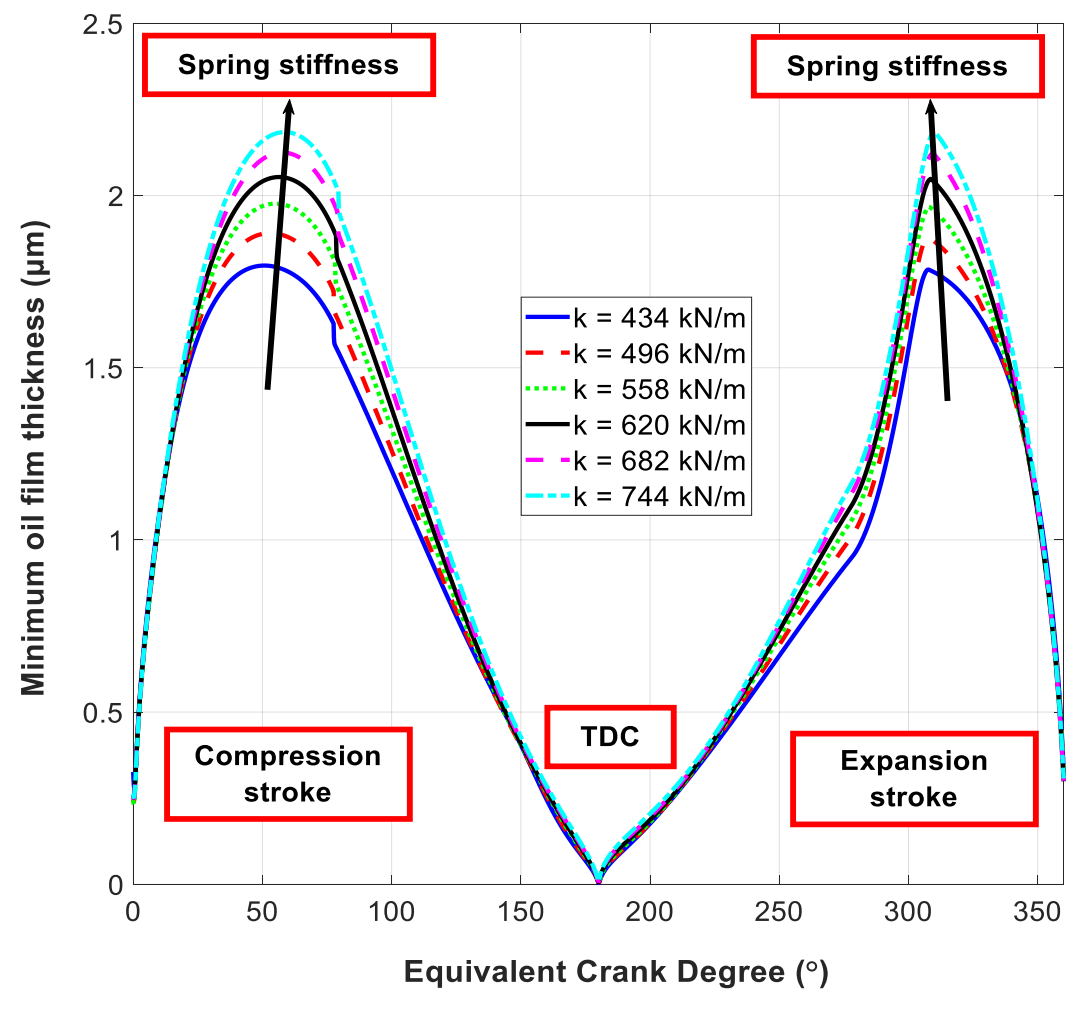

Figure 49: Variation in the minimum oil film thickness distribution for a single cylinder FPEG with increasing spring stiffness. 
Due to the variations in the oil film thickness, the frictional power losses were also varied with increasing spring stiffness. The variations in the frictional power losses with increasing spring stiffness are shown in Figure 50. From Figure 50, the piston ring frictional power losses were increased with increasing spring stiffness. This was due to the increase in frequency and translator velocities with increasing spring stiffness. On an average, the power losses were increased by $0.2 \mathrm{~W} / \mathrm{kN} / \mathrm{m}$ for both the FPEG systems. In terms of percentage, the friction losses were increased from $12.3 \%$ to $17.8 \%$ of indicated power as the spring stiffness was increased from $434 \mathrm{kN} / \mathrm{m}$ to $744 \mathrm{kN} / \mathrm{m}$. In other words, the conversion inefficiency was increased by $5.5 \%$ with increasing the spring stiffness from $434 \mathrm{kN} / \mathrm{m}$ to $744 \mathrm{kN} / \mathrm{m}$.

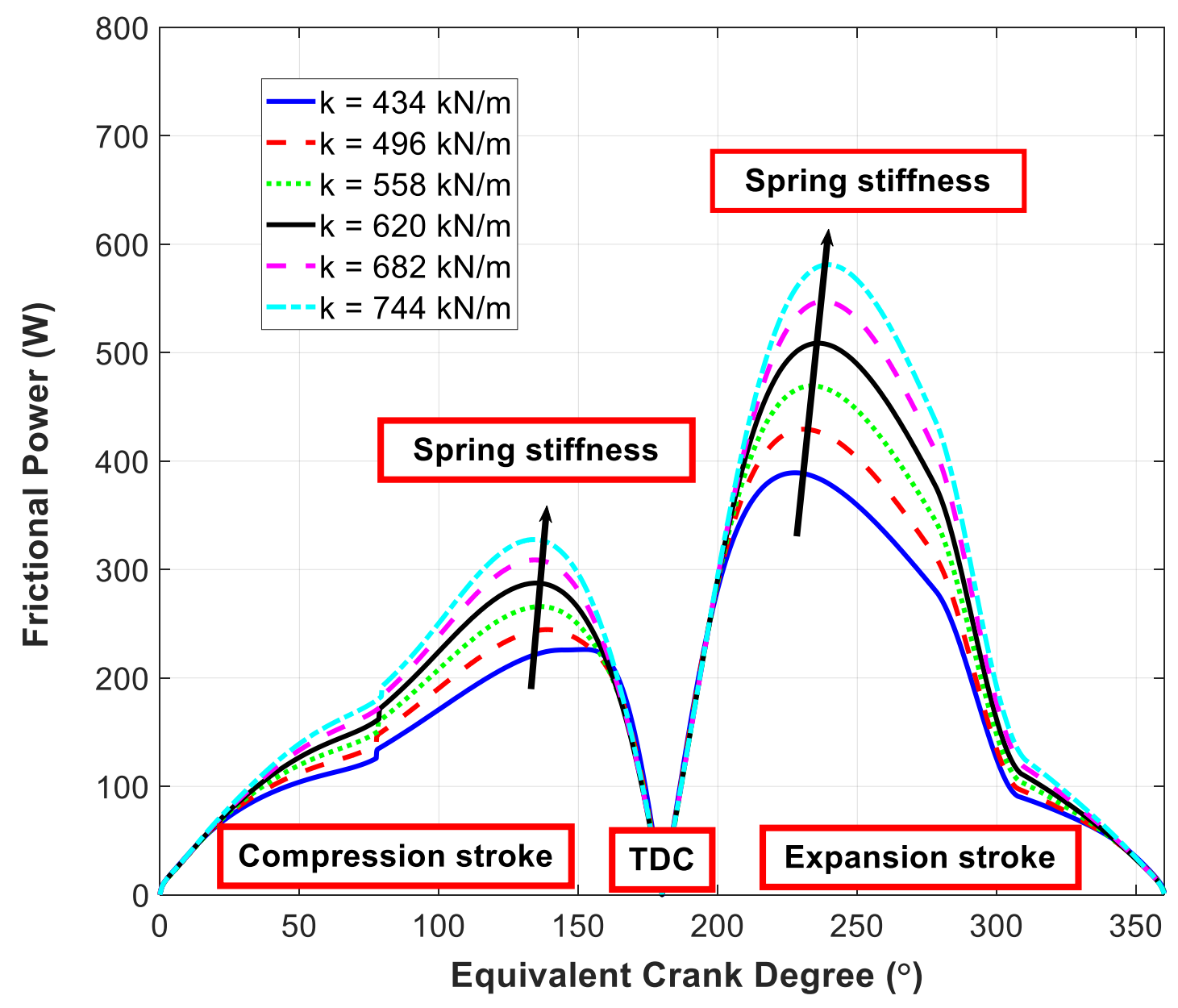

Figure 50: Variation in the piston ring frictional power losses for a single cylinder FPEG with increasing spring stiffness. 


\section{EXPERIMENTAL FPEG PROTOTYPE DEVELOPMENT}

This chapter described the construction, experimental evaluation, and analysis of a prototype under development at WVU. It was a $1 \mathrm{~kW}$, natural gas fueled prototype with an SI combustion mode. The single cylinder design was designed for a CHP system at the residential level. The prototype FPEG system employed a two-stroke loop scavenging method for the gas exchange process. The fuel was delivered in two ways. In a fumigated operation, the fuel was continuously delivered into the intake port. In other words, the fuel flow was continuous rather than an intake port injection. In a direct-injected operation, the fuel was directly injected into the cylinder at a pressure of 4 bar. The stiff flexural springs were designed and added to the prototype system. The springs improved the operating frequency and power output of the system. The electrical power was extracted from the coil windings due to the reciprocated piston operation. In a CHP system, this electric power was used to power the appliances at the residential level.

The experimental single cylinder FPEG system was fabricated, assembled, and evaluated at WVU. The prototype FPEG system under development at WVU was shown in Figure 51. The essential components of the prototype FPEG system included an engine cylinder assembly, mechanical springs, LEM, and a translator rod. The engine cylinder was mounted on the left side in Figure 51. The LEM was mounted in the center. The mechanical springs were packed inside the central casing. The translator rod connected the piston, springs, and LEM. A modified Husqvarna Zenoah G340RC engine cylinder was used in the prototype FPEG system. The engine section consisted of a cylinder, piston assembly, virtual crankcase, intake, and exhaust systems. A piston with a single piston ring located internally in the engine cylinder. A compression volume played the same role as the crankcase compression in the CSE system. This compression volume with an intake port was located below the engine cylinder. Moreover, an intake port was fitted with a reed valve. This reed valve prevented the backflow of fuel-air mixture from crankcase volume to the intake system. A spark plug was mounted in the cylinder head. The intake and exhaust systems were mounted to the intake and exhaust ports of the engine.

The fuel and air were delivered to the crankcase compression volume in the fumigation operation. The mixture was delivered to the combustion chamber through a set of scavenging ports on the cylinder liner. A loop scavenging design with exhaust and scavenging ports was used for the system gas exchange process. These ports were at fixed positions on the cylinder liner. The opening and closing of these ports were defined by the piston displacement. In other words, the 
scavenging process was coupled directly to the transient piston dynamics. With the variation in the dynamics, the FPEG system also exhibited variations in port operation durations. These variations further led to the variations in the trapped fuel and air mixtures in the cylinder every cycle.

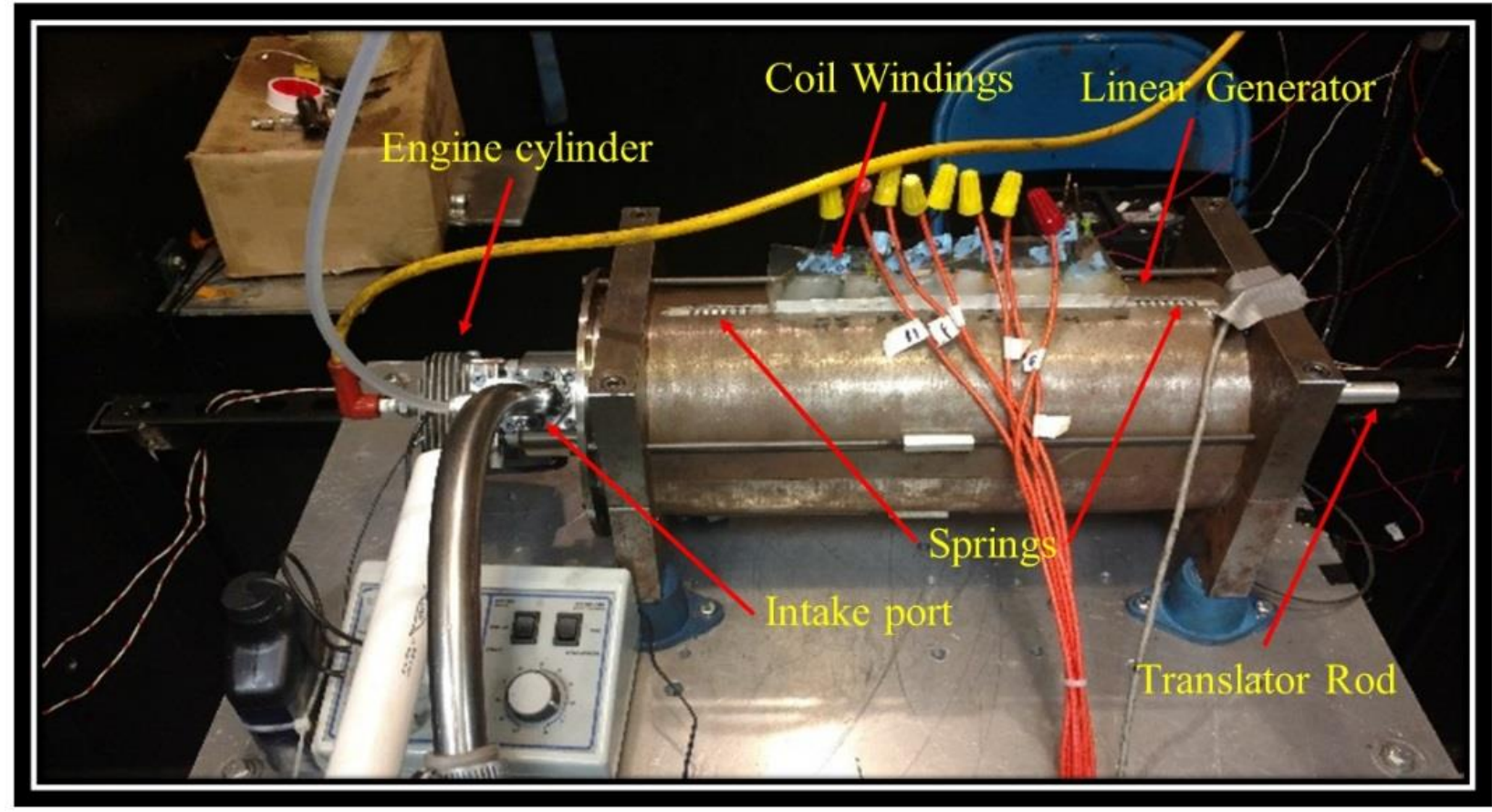

Figure 51: Experimental FPEG system under development at WVU.

The LEM section of the FPEG comprised two main components (i.e. reciprocating component \& stator component). A tubular drum with permanent magnets acted as a reciprocating component. This reciprocating drum was connected to the translator rod. The custom-designed copper coil winding assembly acted as a stator component. The winding assembly was mounted inside the FPEG system casing. The mechanical springs were mounted on either end of the reciprocating tubular drum. Before the starting of the FPEG system, the mechanical springs were in their resting position. In other words, they were neither compressed nor expanded. However, during the FPEG operation, these mechanical springs were compressed and expanded continuously. The magnitude of deflection (i.e. compression and expansion) was governed by the forces and losses in the system. A translator rod was employed in the design to connect the engine piston, springs, and LEM reciprocating component. It was connected to an engine piston at one end, and springs and the LEM reciprocating component in the central section. The piston assembly, translator rod, 
mechanical springs, linear position sensor, and a reciprocating tubular drum were the only noteworthy reciprocating components.

\subsection{Resonant starting process}

The starting of the prototype system was achieved by a combination of three methods employed simultaneously. The first method was the operation of a central mounted LEM as a motor. The LEM operated as a motor provided the necessary linear thrust to the translator rod. Moreover, the LEM provided the motoring force on consecutive starting strokes. This was attained by means of four IGBT's operated on two legs. The motoring force was provided on consecutive strokes because the thrust force was not sufficient to build energy when provided on alternate strokes. The alternative stroke thrust method was explained in the next paragraph. This motoring process was continued until the combustion favorable conditions were reached. The combustion favorable conditions in the experimental process were defined in terms of a target stroke length, and hence a target compression ratio. Moreover, these combustion favorable conditions were attained gradually rather than a single stroke. This gradual increase in the effective stroke length was mainly because of the resonance phenomenon. The resonance characteristics were mainly exhibited by the engine cylinder and mechanical springs.

The second method used the energy stored in the springs to compress the cylinder contents. With the addition of stiff springs, the LEM was managed to provide the linear thrust only on the alternative strokes. In this case, the energy from the springs and LEM in alternative stroke was sufficient to start the FPEG system. Consequently, the LEM thrust force was provided only on the alternative strokes. This linear thrust on alternative strokes was attained using four IGBT's operated on two legs. However, for this method, only two IGBT's were turned on at any time. The linear thrust from the LEM was provided to compress the stiff springs in alternative strokes. Finally, if the energy was not sufficient enough, the LEM thrust force was provided on consecutive strokes by using a control strategy. In the experimental FPEG system, the linear thrust was provided to the springs at TDC during the expansion stroke of the engine. At the end of the expansion stroke, the stiff springs were compressed. Moreover, the motoring energy remaining after the losses was stored within the spring system. This stored energy was used to compress the cylinder contents in the compression stroke. 
Depending upon the magnitude of linear thrust, the energy was built in the system either in one stroke or gradually. In the experimental FPEG system, a gradual approach (mechanical resonance) was used for the starting process. The main reason for considering the mechanical resonance process was the non-feasibility of the one-stroke method. The non-feasibility was due to a high energy input required from the LEM. As a result, the resonance process or the gradual approach was used for the starting the experimental FPEG system.

The third method was the assistance of combustion in case of extremely stiff springs. This method was used only if the LEM failed to provide the energy to start the spring-dominant FPEG system. In this method, the cylinder contents were ignited near TDC and at low compression ratios. In other words, the mixture was ignited at TDC to provide the combustion thrust force. This combustion force provided the additional linear thrust to the springs. This process was repeated until the FPEG system reached stable conditions. The stable conditions were defined in terms of a target trapped compression ratio. After that, the linear thrust from the LEM was turned off gradually. Moreover, the ignition timing was also adjusted to an optimized ignition timing. Finally, the FPEG system was switched to the generating mode once the stability conditions were reached.

\subsection{Stable combustion process}

The combustion system in the prototype FPEG system at WVU was designed for an SI combustion mode. Natural gas was used as fuel. Natural gas with $86 \%$ methane, $12 \%$ ethane, $1 \%$ propane, $0.5 \%$ nitrogen and $0.5 \%$ carbon dioxide composition was selected. The natural gas was chosen for several reasons: 1 . The interest of DOE ARPA-E with natural gas supplied to homes, 2. Reduced exhaust gas emissions relative to gasoline 3. Increase in the engine efficiency through high compression ratios (higher knock resistance than gasoline) and 4. Low cost. The combustion system of the prototype FPEG system was made up of multiple subsystems. These subsystems were the air and fuel delivery, spark, and air cooling systems.

Depending upon the requirements, the fuel was delivered either to the crankcase compression volume or into the combustion chamber. In a fumigated operation, the fuel was delivered through a reed valve attached to the crankcase compression volume. In a direct-injected operation, the fuel was delivered into the combustion chamber. In the fuel delivery system, the natural gas was supplied to the engine from the gas cylinders by using a mass flow controller. The supply lines 
were connected from the gas cylinders to the mass flow controller and then to the FPEG system. A mass flow controller placed in-between the gas cylinder and the FPEG system measured the amount of fuel delivered to the FPEG system. Moreover, a buffer volume was considered in a direct-injected operation. It was placed in-between the mass flow controller and the injector. This buffer volume reduced the pressure fluctuations due to the operation of a fuel injector. The initiation and the pulse width of the fuel injector were controlled by using an in-house controller. Initially, these values were fixed before the starting of the FPEG system. Moreover, a dead band was provided for these variables in the control system. During the stable generation process, these values were modified manually within the dead band for stable FPEG performance. Depending upon the cycle-to-cycle variations, the start of injection and injection pulse width were either advanced or retarded. In other words, the injection operation parameters were not optimal due to cycle-to-cycle variations. A laminar flow element was used to calculate the amount of air supplied to the crankcase. The lubricating oil was metered directly into the virtual crankcase. This was attained by using a piezo resistive pump. The schematic diagram of the prototype FPEG system is shown in Figure 52.

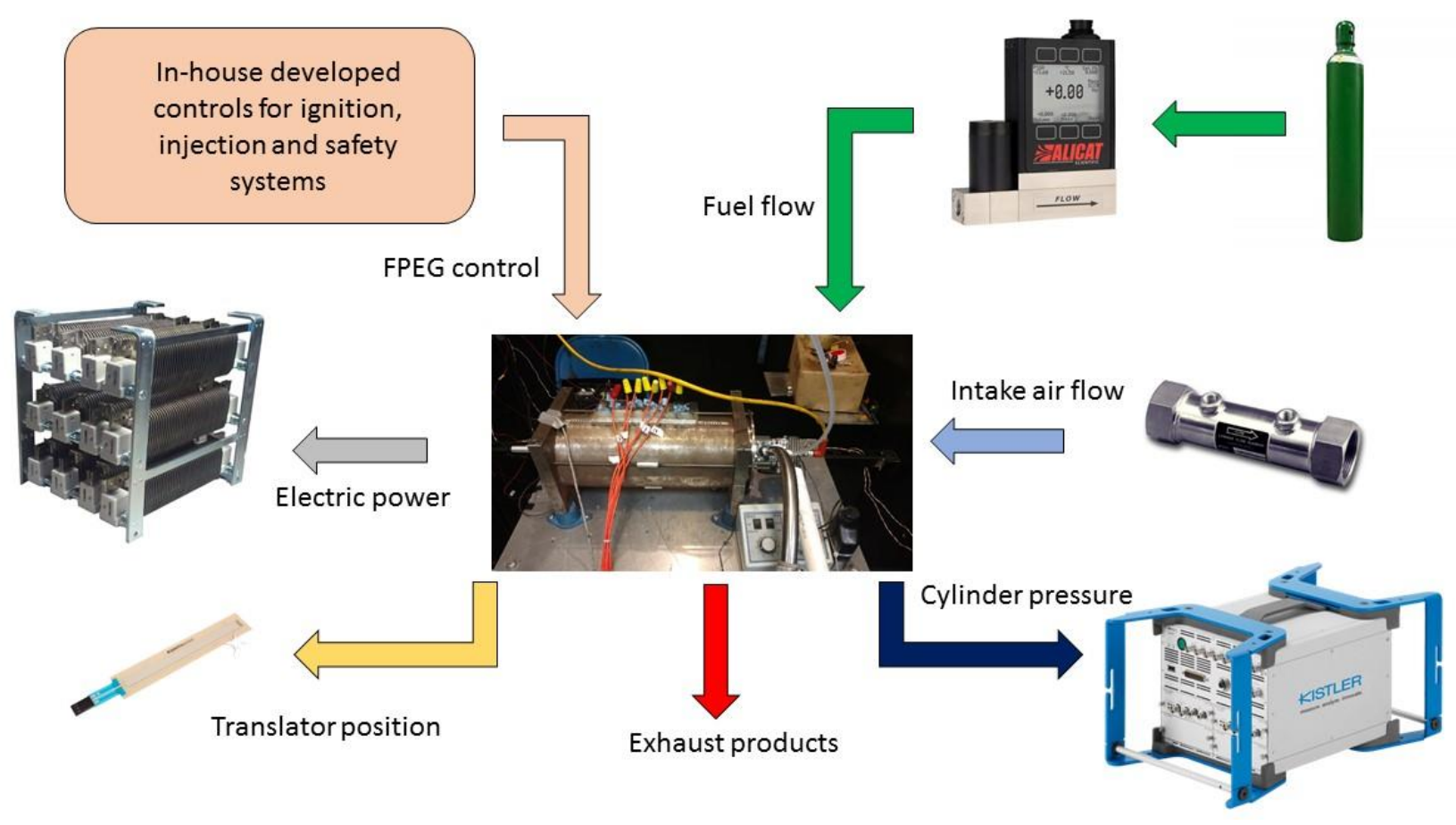

Figure 52: Schematic diagram of prototype FPEG system at WVU. 


\subsection{Data acquisition systems}

Several measurements were used to evaluate the FPEG performance characteristics. The measurements were the translator position, combustion pressures, fuel flow rate, ignition timing, injector parameters, LEM current, and voltage readings. The first and foremost important measurement was the translator position in the cylinder. The translator position was recorded with the help of a linear potentiometer. The potentiometer worked on the principle of sliding contact voltage divider. The sliding contact of the translator with the potentiometer divided the fixed input voltage between the two ends. This voltage was later converted into the translator position. A low pass filter was applied to the translator position measurement. This low pass filtering eliminated the noise and disturbances in the translator position. The readings from the potentiometer were further post-processed using a MATLAB® script. The post-processing script calculated the instantaneous cylinder volume, dead center locations, and trapped compression ratio.

The cylinder combustion chamber pressure was measured by using a Kistler piezoelectric pressure transducer. The sensor was of type 6054B with a 0-250 bar measuring range. This pressure transducer was used in conjunction with the Kistler charge amplifiers. The accuracy of the pressure transducer was found to be 1.5 bar or $0.5 \%$ of the total measurement range. The accuracy was found based on the sensor specifications provided by the Kistler. The same method was used for determining the accuracies of all sensors used in the system. In addition, the accuracy varied linearly. The cylinder pressures in this dissertation were accurate within $+/-0.13$ bar or $0.04 \%$. For example, for a measurement of 25 bar, the actual pressure was in-between 24.87 to 25.13 bar. The cylinder pressure measurements were referenced to the absolute pressure while post-processing the results. The referenced pressure was calculated when the FPEG system was in the resting phase. Moreover, both the exhaust and intake ports were opened in the resting phase. In addition to the cylinder pressure, the crankcase compression pressure was also measured. This was done by using a Kistler piezoresistive pressure transducer of type 4007B with a 0-5 bar measuring range. This pressure transducer was also used in conjunction with the Kistler charge amplifiers. The accuracy of this pressure transducer was found to be 0.005 bar or $0.1 \%$ of the total measurement range. Moreover, the accuracy varied linearly. For the crankcase pressures in this dissertation, the pressures were accurate within $+/-0.002$ bar or $0.04 \%$. In this dissertation, the time delay in the pressure measurements was assumed negligible. 
The intake airflow into the system was measured by using a Meriam 50MW20 laminar flow element of 565 SLPM (Standard Liter Per Minute) capacity with a differential pressure gauge. The accuracy of the intake airflow was $0.5 \%$ of reading or $0.1 \%$ of the total range (whichever is greater). In terms of SLPM, the intake airflow was accurate within +/-0.85 SLPM for all the cases. The fuel flow rate was measured using an Alice ${ }^{\circledR}$ MC-20 series mass flow controller with a 0-20 SLPM measuring range. The mass flow controller was calibrated to the composition of natural gas fuel used in the experimental system. This calibration avoided the errors due to MFC correction factors. Based on the specifications, the fuel flow measurements were accurate within $+/-0.01$ SLPM or $0.05 \%$ of the total measurement range.

The ignition timing in the experimental FPEG system was defined in terms of time after BDC. Initially, the experimental control system identified the location of BDC. This was done based on the sign change of translator velocity. After identifying the BDC location, a timer was used to activate the ignition system. This was done using a predefined ignition timing after BDC. This predefined ignition timing in seconds was changed manually. Moreover, with nearly constant frequency operation defined by the stiff springs, the ignition timing was maintained the same for all the cycles. In other words, with the variations in stroke length and frequency, the ignition timing was either retarded or advanced. In other words, the ignition timing was not at an optimum location due to cycle-to-cycle variations.

The electrical current and voltage outputs from the LEM system were also measured. This was done using a hall effect current transducer and a voltage transducer. They had a measuring range of $0-20 \mathrm{~A}$ and $0-500 \mathrm{~V}$. The load resistance and capacitance were measured. This was done using the resistance and capacitance ratings on the load bank of resistors and capacitors. Finally, the cylinder head, cylinder liner, virtual crankcase, and LEM temperatures were measured by using Type-K thermocouples. Omega surface thermocouples with self-adhesive backing and a range of $273 \mathrm{~K}$ to $450 \mathrm{~K}$ were used. The temperatures were recorded by using a separate temperate acquisition system. Other than temperatures, all other measurements were recorded using a Kibox® data acquisition system.

\subsection{Data analysis}

The dynamics and combustion data were recorded on a time basis at a sampling frequency of $0.5 \mathrm{MHz}$. The heat release rate, IMEP, mass fraction burned (MFB), and other combustion 
statistics were calculated externally. They were calculated using a script composed in the MATLAB $\circledast$ software. This script interpreted the voltage measurements, performed calculations, and scaled the outputs to the respective engineering units. Moreover, the script also calculated the thermodynamic cycle measurements. This includes the dead center locations, port operating and geometry locations. The translator position and engine geometry data were used for these calculations. A low pass filtering was applied to the translator position data. This was done for noise rejection when locating these events. Finally, the ignition timing, frequency, and equivalent crank angle degrees were determined. This was based on the time between the two successive BDC positions.

The instantaneous cylinder volume and trapped compression ratios were calculated using the filtered position data and EPC location. The experimental MFB was calculated based on the normalized net pressure calculations in Section 2.2.4. The normalized net pressure included the pressure variations due to heat transfer and mass loss past the ring. The reason for using the normalized net pressures was to eliminate the effect of pressure due to volumetric changes. The normalized first derivate of the MFB was treated as the heat release rate in this dissertation. In other words, the first derivative of the MFB defined the velocity of the combustion process. Due to instability and cycle-to-cycle variations, the calculated experimental MFB does not follow a smooth distribution. The supplied fuel energy input was calculated based on the fuel flow rate, the LHV of the fuel, and operating frequencies respectively.

In addition to these, the instantaneous LEM electric power output was also calculated. This was done by multiplying the instantaneous voltage, currents, and a power factor. The average value of the electrical power output on a cycle-to-cycle basis was calculated by integrating the instantaneous power. The indicated work was calculated by using the area under the cylinder pressure and volume diagram. The indicated power was calculated by multiplying the indicated work and operating frequency. Finally, the indicated and FPEG system efficiencies were calculated.

\subsection{Experimental results \& model validation}

This section reported the results obtained from a fumigated experimental FPEG system. The system generated $550 \mathrm{~W}$ of electricity with an indicated efficiency exceeding $13.8 \%$. Moreover, these results were also used for the validation of the numerical model. The validation of the 
numerical model developed in MATLAB®/ Simulink was pursued in two approaches. The first approach validated the numerical model during the starting mode. This approach allowed for the validation of the LEM motor and spring model. Moreover, it excluded the engine combustion out of the validation in this case. Finally, the accuracies of the modeled dynamics and combustion characteristics were calculated.

The second approach validated the numerical model during the stable generating mode. In this stage, every submodel was validated individually. Moreover, the accuracy of the modeled dynamics and combustion characteristics was calculated. At first, the FPEG system dynamics were validated. After FPEG dynamics, the next step validated the cylinder thermodynamics model. The curve fitting and mathematical techniques available in MATLAB® were used for the validation of the thermodynamics model. In other words, the experimental findings were used to refine and validate the numerical model. For example, a mathematical formulation based on the experimental cylinder pressure was used to formulate the model-predicted heat release rate. Similarly, the crankcase pressure was provided to the numerical model using lookup tables. Later, the LEM load generation characteristics were validated. Finally, the qualitative comparisons were made between model-predicted and experimental dynamics and combustion characteristics.

\subsubsection{Resonance starting process results \& validation}

In this section, the modeling results from the mechanical resonant starting process were presented. The results were reported based on experiments and numerical model. The experimental FPEG system was configured with stiff springs was operated at a frequency of 73.5 $\mathrm{Hz}$. The rise in the translator displacement at the startup of experimental FPEG prototype is shown in Figure 54. It is evident from Figure 54 that within $0.6 \mathrm{sec}$ the amplitude of the displacement grew to $20 \mathrm{~mm}$. Therefore, the constant linear thrust force on consecutive strokes was sufficient to reciprocate the FPEG system in small amplitudes. Moreover, a minute energy was wasted for the friction and spring losses in the compression process.

In Figure 53, the FPEG system reached the EPO in 21 cycles after it was started from its neutral point location. After 21 cycles, the amount of energy stored in the system was found to be $2.5 \mathrm{~J}$. This energy corresponded to the $4 \%$ of the maximum spring energy storage. The FPEG system reached the SPO in additional 17 cycles after reaching the EPO. This energy present in the system at the time of SPO was found to be $19.9 \mathrm{~J}$. In other words, the energy was increased by 
$700 \%$ when compared to the energy at the EPO. Finally, the FPEG system reached $67 \%$ of the effective stroke (i.e. $20 \mathrm{~mm}$ ) in 49 resonant cycles. At the end of the starting process, the FPEG system stored $27.5 \mathrm{~J}$ of energy to reach $67 \%$ of the effective stroke.

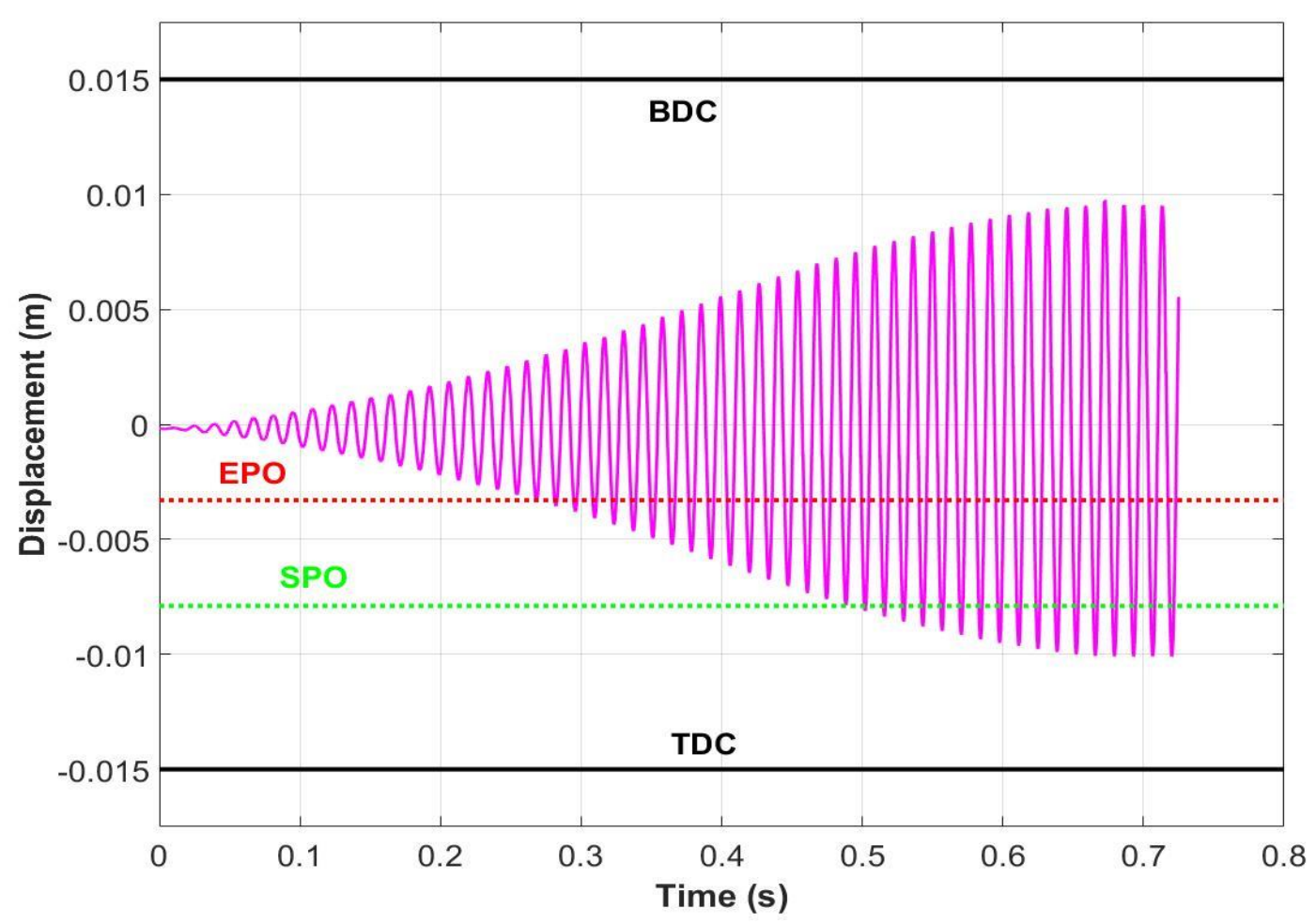

Figure 53: Translator displacement of FPEG system during the starting process.

The variation in the translator velocity as a function of time and position is shown in Figure 54 and Figure 55. Figure 54 demonstrates that the energy stored in the resonant mechanical springs caused the translator velocity to rise from cycle-to-cycle. Moreover, the translator velocity was zero at the initial phases of starting. This was due to the static friction of the engine pistontranslator rod-spring assembly. Since the static friction was higher than the LEM motoring force, the system was at rest. Once the LEM motoring force exceeded the static friction, the FPEG started to reciprocate. Based on this, the starting process was divided into two stages. The first stage refers the stage when the LEM thrust force was below the static friction. The second stage referred to the energy buildup in the springs and FPEG system. This energy buildup in the system caused the velocity and position to grow from cycle-to-cycle as evident from Figures 53, 54 and 55 . 


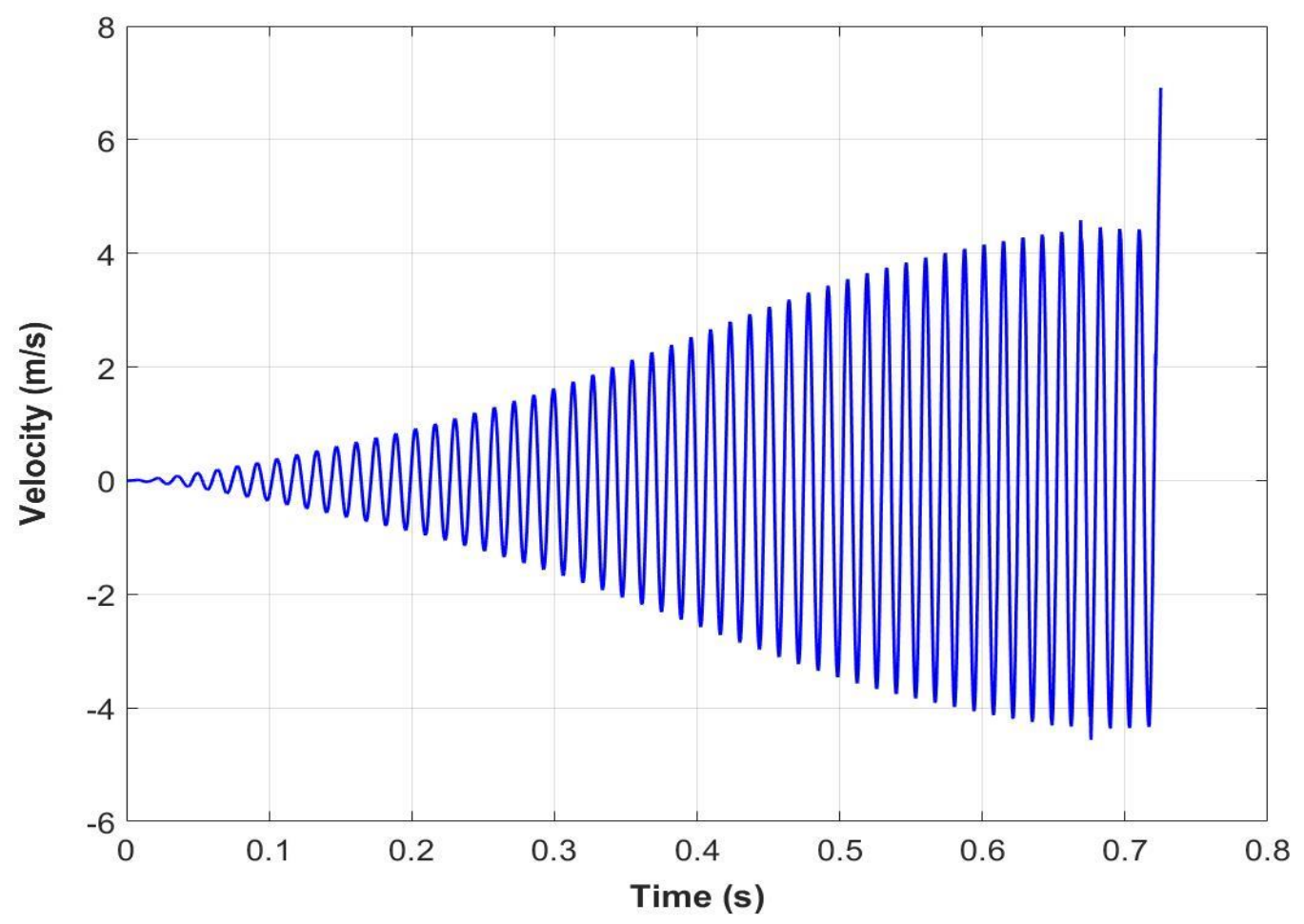

Figure 55: Translator velocity of FPEG system during the starting process.

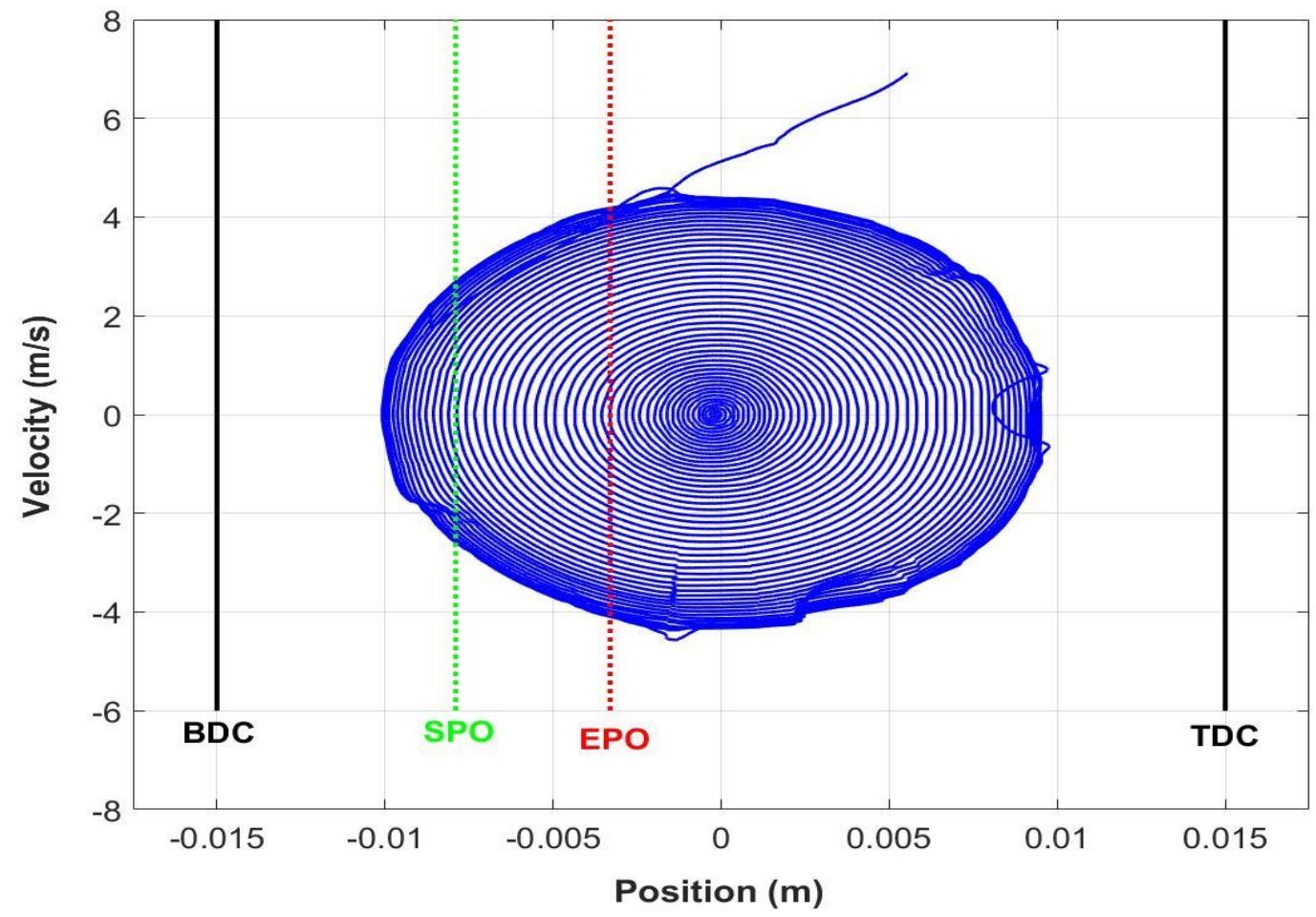

Figure 54: Velocity versus position distribution of FPEG system during starting process. 
From Figure 55, two different regions were identified in the velocity versus position plot. The first region is the dark oval region in the center. The resonance of the stiff springs was minimal in this region. This was because the supplied LEM motor force was on par with the static friction. The second region corresponds to the sparsely spaced velocity profile. In this region, the velocity was increased from $0 \mathrm{~m} / \mathrm{s}$ to $4.25 \mathrm{~m} / \mathrm{s}$ within $0.6 \mathrm{sec}$. This was due to the energy storage in the springdominant FPEG system. Once the translator displacement reached $20 \mathrm{~mm}$, the fuel was delivered into the system. Later, the fuel and air mixture were ignited. With the combustion of fuel, the velocity of the translator was further increased from $4.25 \mathrm{~m} / \mathrm{s}$ to $6 \mathrm{~m} / \mathrm{s}$. The increase in the velocity due to combustion was evident from the line deviating the spiral in Figure 55. Further, with the increase in translator rod velocity, the compression ratio was also increased. The increase in the compression ratio was visualized by using the cylinder pressure distribution shown in Figure 56.

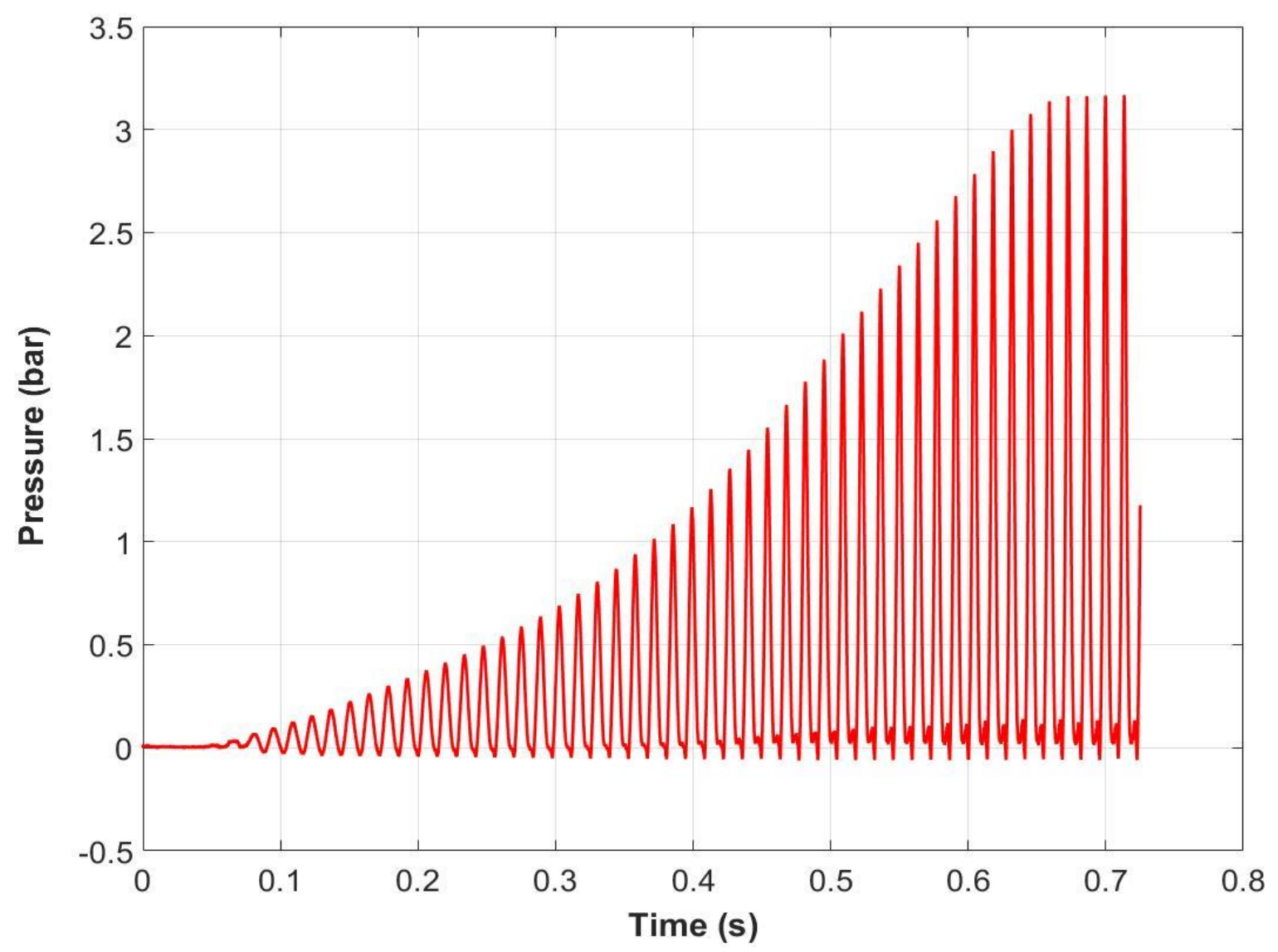

Figure 56: Absolute cylinder pressure of FPEG system during starting process. 
Starting submodel validation

The experimental starting process of the FPEG system under development was modeled in the numerical model. The mathematical equations corresponding to the starting process were presented in Section 2.2.8. In the experimental FPEG system, the system was operated at a frequency of $73.5 \mathrm{~Hz}$. In the numerical model, it reciprocated at an operating frequency of 74.5 $\mathrm{Hz}$. The frequency was dependent upon the stiff spring stiffness, moving mass, and the losses in the system. Though the stiff spring stiffness was predefined, the losses and vibrations due to external mounting were not considered. As a result, the numerical model exhibited slightly high operating frequency. The comparison of experimental and modeled dynamics during the starting process is shown in Figure 57.

From Figure 57, the modeling results agree with the experimental dynamics during the starting process. Initially, the peak of translator displacement in every cycle of the starting process was calculated. Later, the error was estimated by comparing the displacement peaks from cycle-tocycle. Finally, the average error in the translator displacement peaks was used to define the accuracy of the numerical model. Based on the calculations, the error in the translator displacement between the numerical model and experimental system was found to be $5.8 \%$.

From Figure 57, it is evident that the variations in the velocity were more dominant when compared to the displacement. The numerical model over predicted the translator velocity as shown in Figure 57. The error calculation for the velocity used the same method as that of translator displacement. In this case, the numerical model over predicted the translator velocity by $9.5 \%$ when compared to the experimental velocity. In other words, the numerical model predicted the displacement and velocity with $94.2 \%$ and $90.5 \%$ accuracies respectively. Figure 58 shows the variation in the modeled and experimental cylinder pressure.

From Figure 58, in the initial cycles, the numerical model overpredicted the cylinder pressure. After a few initial cycles, the numerical model predicted the cylinder pressure accurately. Similar to the previous cases, the peak cylinder pressures were used for the error calculation. Based on that, the modeled cylinder pressure was deviated by $10.9 \%$. In other words, the cylinder model was $89.1 \%$ accurate during the starting stage. Overall, there exist slight deviations in the modeled dynamics and cylinder pressures. However, the numerical model accuracy was greater than $85 \%$ 
for all the FPEG characteristics during the starting phase. These results provided the confidence to use the starting submodel for further investigations.
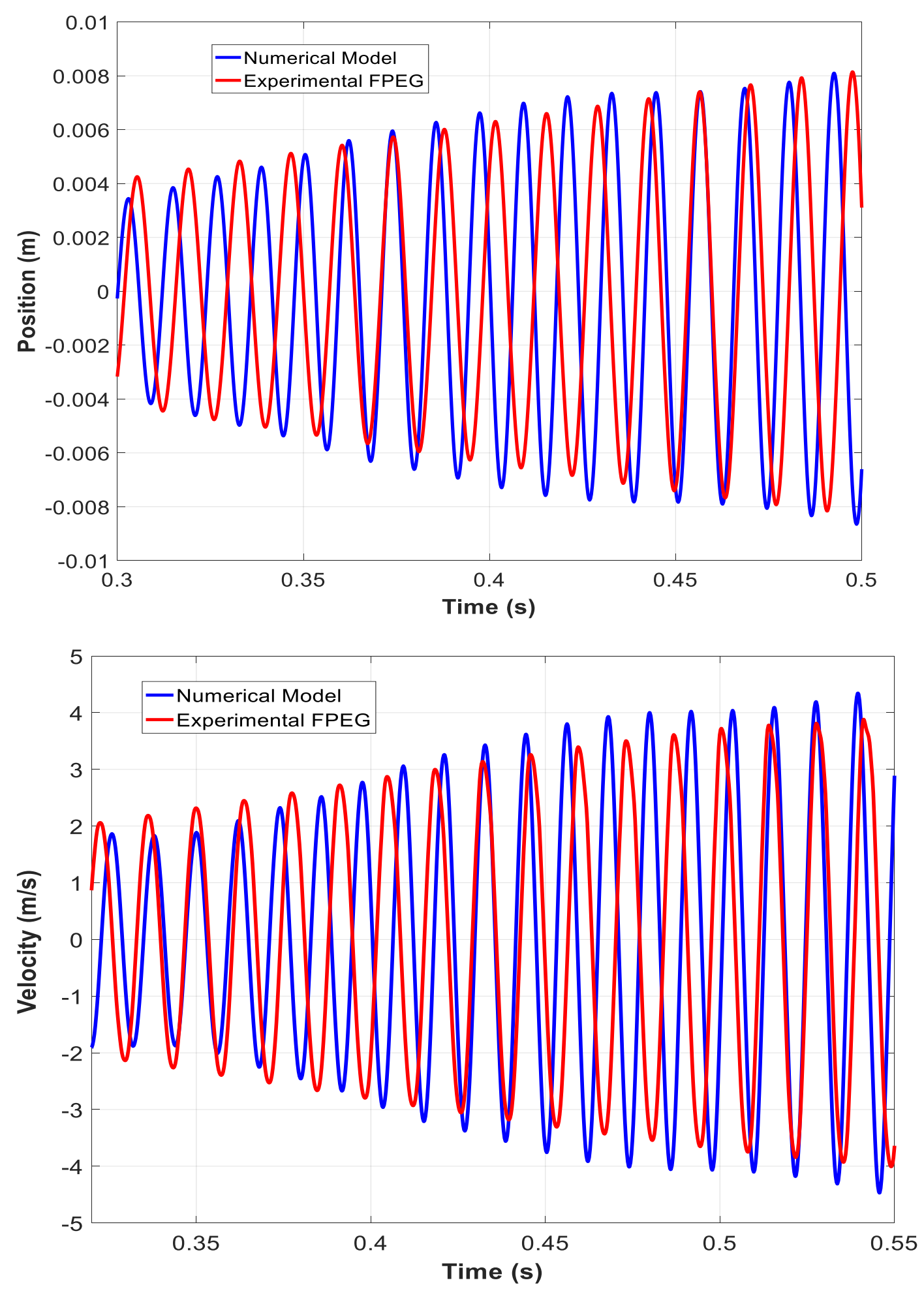

Figure 57: Experimental and modeled position and velocity during the FPEG starting process. 


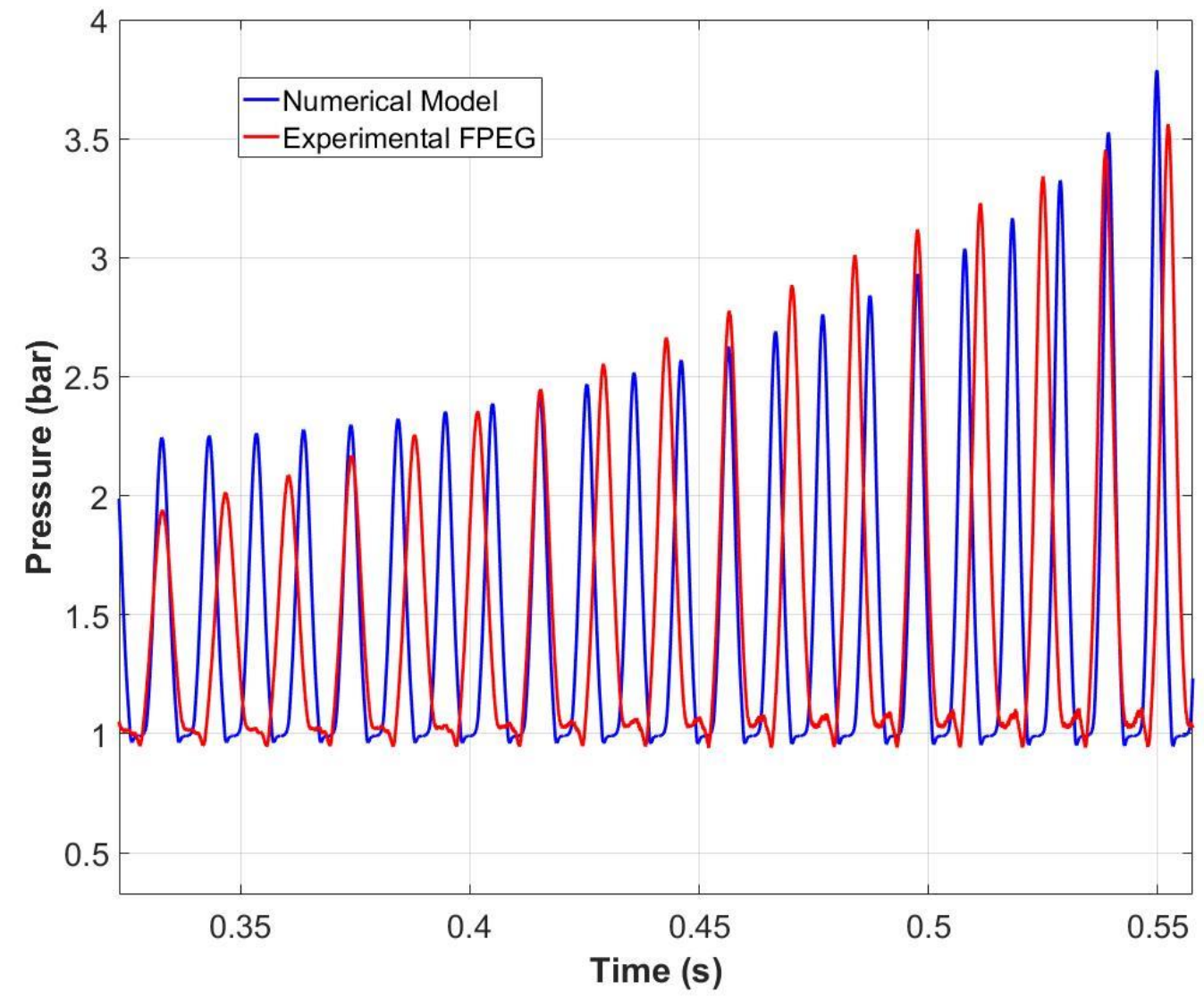

Figure 58: Experimental and modeled cylinder pressure during the FPEG starting process.

\subsubsection{Generator phase results \& validation}

This section reported the experimental results from the combustion tests of the fumigated FPEG system. The experimental prototype generated $550 \mathrm{~W}$ of electricity with indicated efficiencies exceeding $13.8 \%$. Various sensor measurements were used for defining the FPEG performance. They include the position, cylinder pressures, fuel flow rate, ignition timing, crankcase pressure, load current, and load voltage. The analysis procedure and the data acquisition systems used were presented in Sections 3.3 and 3.4.

For the experimental combustion tests, the FPEG system was made to run without any resonant intake and exhaust systems. The intake air of 106.2 SLPM was delivered to the combustion chamber through the crankcase. This intake airflow was measured by using a laminar flow element with a differential pressure gauge. Based on the actual and theoretical airflow, the 
delivery ratio was calculated. The theoretical airflow was calculated using the effective displacement of the engine cylinder. The engine cylinder was designed for an effective displacement of $30 \mathrm{~mm}$. Based on the calculations, the FPEG delivery ratio was found to be $63.7 \%$ on an average for 800 operating cycles. The poor delivery ratio was mainly because of the reduced stroke length of the experimental system. The experimental FPEG system operated at an average stroke length of $26.8 \mathrm{~mm}$ for 800 cycles. Moreover, with stiff springs, the stroke length was changed by only $+/-0.2 \mathrm{~mm}$ from the average for all the operating cycles.

Natural gas of 11.50 SLPM was fumigated directly into the crankcase compression volume through a reed valve. The ignition timing was adjusted manually. Based on the combustion data, the ignition timing was between $4.95 \mathrm{~ms}$ and $5.15 \mathrm{~ms}$ after BDC. With the cycle-to-cycle variations, the ignition timing was either retarded or advanced. In other words, the ignition timing was not optimal due to cycle-to-cycle variations. Due to a mismatch of energy from the engine to the LEM, the springs used in the experimental system were subjected to an operating state beyond their design limits. As a result, the springs were broken which further led to a reduction in operating frequency. Hence, an ignition cutoff system was used for the combustion tests. This ignition cutoff system was employed to prevent the failure of stiff springs in the system. The translator displacement was used for the activation of the ignition cutoff system. In this case, the ignition system was turned off when the stroke length went beyond $28 \mathrm{~mm}$. However, the FPEG system operated continuously without activating the ignition cutoff system. This was because the FPEG system was operated at a displacement lower than the cutoff displacement. Moreover, the generator load was sufficiently high to absorb all the engine output. In other words, the engine was not overpowered relative to the generator.

For the model validation, initially, the geometric conditions were provided to the model. Next, the numerical model was made to run for a given stroke and compression ratio. This stroke length and the compression ratio were defined based on the experimental system. Moreover, the port locations based on the initial position were maintained the same. Next, the spring stiffness obtained from experimental testing of flexure springs was provided to the numerical model. The flexure springs were tested in the Material Testing Systems (MTS) compression and expansion machine. The flexure springs were compressed and expanded in the MTS machine to $15 \mathrm{~mm}$ on either side of the neutral position. The force and the displacement data obtained from the MTS testing machine was used to calculate the spring stiffness. Moreover, this calculated spring stiffness was provided to the spring force submodel. After spring stiffness, the damping coefficient 
was estimated from the ring down tests. In addition to these, the translator moving mass was also provided as an input to the model. Finally, the LEM open circuit voltage, heat release rate profile, and crankcase pressures were also provided to the numerical model. The validation of the numerical model was presented in the upcoming section.

\subsubsection{FPEG dynamics}

This section presented the comparison of modeled and experimental FPEG system dynamics for the same operating and geometric conditions. Both, the numerical model and experimental systems were operated at the same frequency of $81.5 \mathrm{~Hz}$. The same frequency was maintained because of accurate spring stiffness, moving mass, and damping coefficient inputs. In the numerical model, the flexure springs were modeled as linear with a constant damping coefficient. However, the springs when installed in the system might or might not be linear. This was due to the losses within the system. The translator velocity plotted against translator displacement is shown in Figure 59.

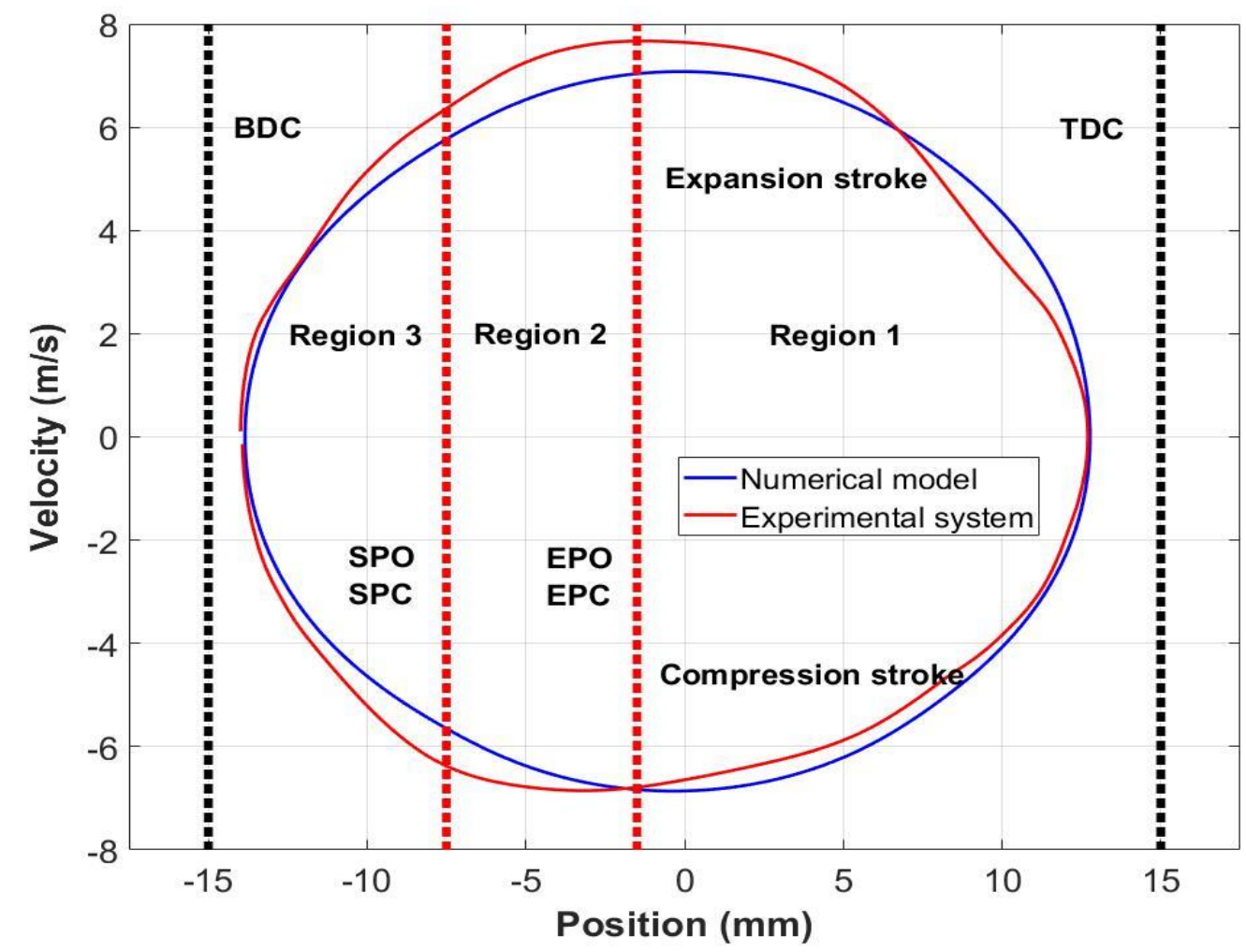

Figure 59: Experimental and modeled velocity and position during the FPEG generating process. 
Similar to the results presented in Section 2.3, a target displacement of $30 \mathrm{~mm}$ was considered while showing the results. The entire area of operation was divided into three regions defined in Section 2.3. In the fundamental case presented in Section 2.3, the effective displacement and trapped compression ratio were $30 \mathrm{~mm}$ and 8.3. In the experimental FPEG system, the effective displacement and compression ratio were found to be $26.8 \mathrm{~mm}$ and 5.3. From Figure 59, the dynamics distribution predicted by the numerical model was not the same as the dynamics distribution of the experimental FPEG system. One main reason for the variation was due to the differences in the stiff springs force profile in the experimental and modeled system. The numerical model assumed springs as ideal compression springs with linear deflection and linear damping. However, in reality, the spring force profile might be linear or non-linear. The deflection profile was influenced by the vibrations and losses in the system. Moreover, the spring stiffness

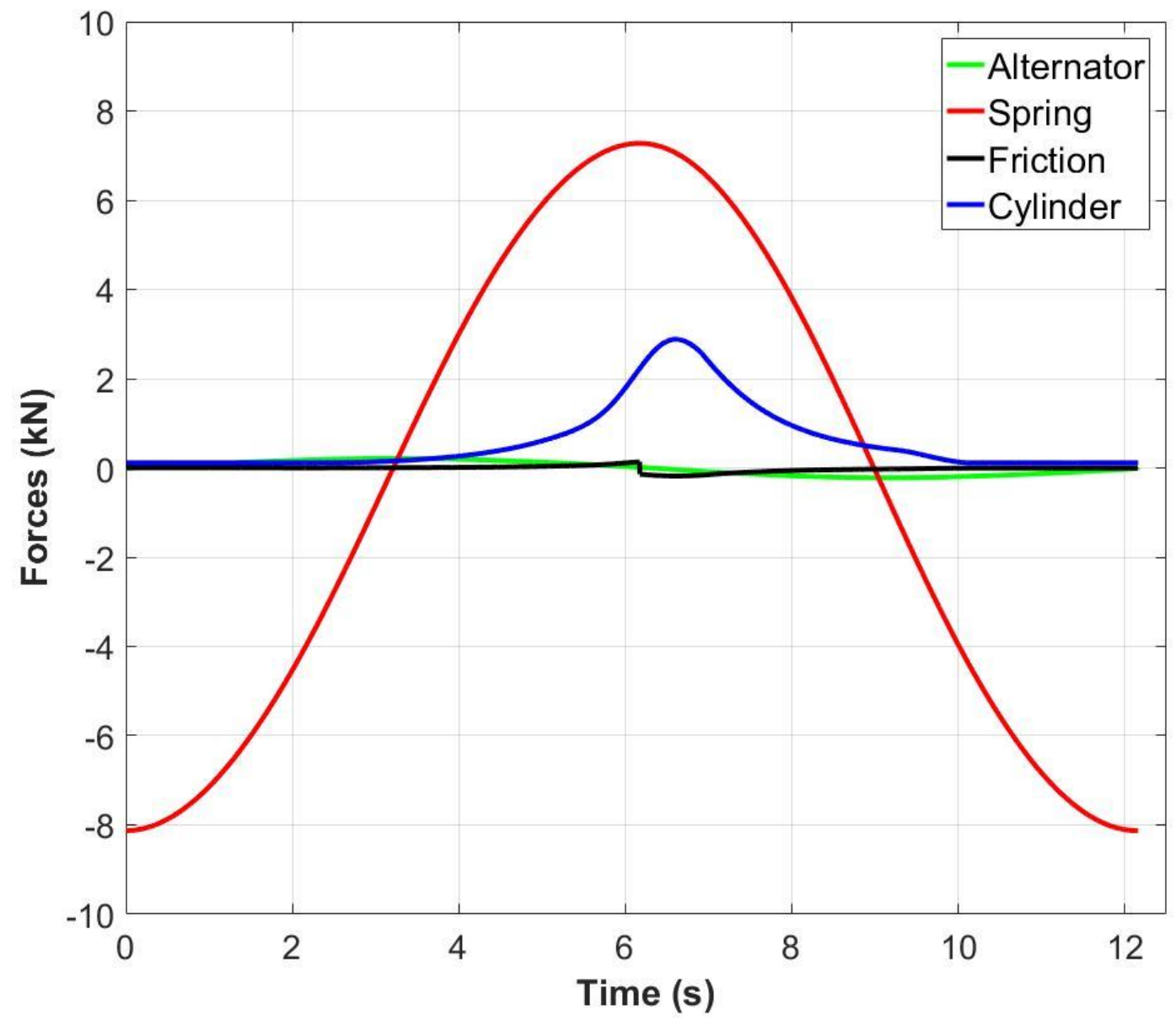

Figure 60: Distribution of forces predicted by numerical model in an FPEG system. 
and damping coefficient inputs were time-averaged parameters. Specifically, the spring stiffness and damping coefficient were obtained as average values for a period of 10 seconds. However, the numerical modeling results were time-based rather than time-averaged results. By way of explanation, the same time-average value can be obtained with different spring force profiles. Due to these differences, the variations were observed in the results. The system of forces acting on the translator rod is shown in Figure 60. From Figure 60, the spring forces were higher than the rest of all the forces acting on the translator. When compared to the peak cylinder force, the peak spring force was $255 \%$ higher. This explained the dominance of the spring forces in defining the dynamics of the system.

Secondly, the displacement was measured by using a linear potentiometer. This potentiometer was placed at the far end of the translator rod. In other words, the translator displacement was assumed the same as the piston displacement. However, the translator rod was connected to the engine piston by using a series of mechanical connections. The lack of rigidity in the mechanical connections (knuckle mechanisms) was also responsible for the variations in the dynamics. This lack of rigidity was evident from the bump in the expansion stroke slightly after TDC. This bump was mainly due to the piston reversal at TDC. The same bump was also seen at the end of the compression stroke. Due to the differentiation of the position data, the variations in the velocity were even worse. Similarly, the acceleration was calculated by the double differentiation of the displacement data. The errors were now propagated into the second order while calculating the acceleration. As a result of the variations were predominant in the acceleration and velocity diagram. The variations in the acceleration and velocity plot are shown in Figure 61. A discontinuity was observed at TDC in Figure 61. This discontinuity was due to the division of cycles in the experimental data. to Figure 59, the same bumps were observed slightly after TDC and BDC in Figure 61.

The deviations in the model predicted and experimental translator dynamics are shown in Figure 62. From Figure 62, it was evident that the differentiation of the position aggravated the error. With velocity and acceleration calculations, the error was propagated to the first and second orders. Initially, at the dead centers, the translator position deviation was close to $0 \%$. However, the major deviations in the translator displacement were observed in the mid-stroke region. These variations were attributed to two factors. The first factor was the interactions of springs with the external mounts. The second factor was the deviations induced by the mechanical connections. From Figure 62, the deviation in the translator displacement was increased from $0 \%$ to $9.1 \%$ from 
TDC to the mid-stroke. Later, the deviation was reduced back to zero at BDC. On average, the position was deviated by $4.0 \%$ from a cycle-to-cycle basis. From Figure 62 , the deviations in the velocity and acceleration were doubled and quadrupled when compared to the displacement deviation. On an average cycle-to-cycle basis, the deviations in the velocity and acceleration were found to be $9.9 \%$ and $16.5 \%$.

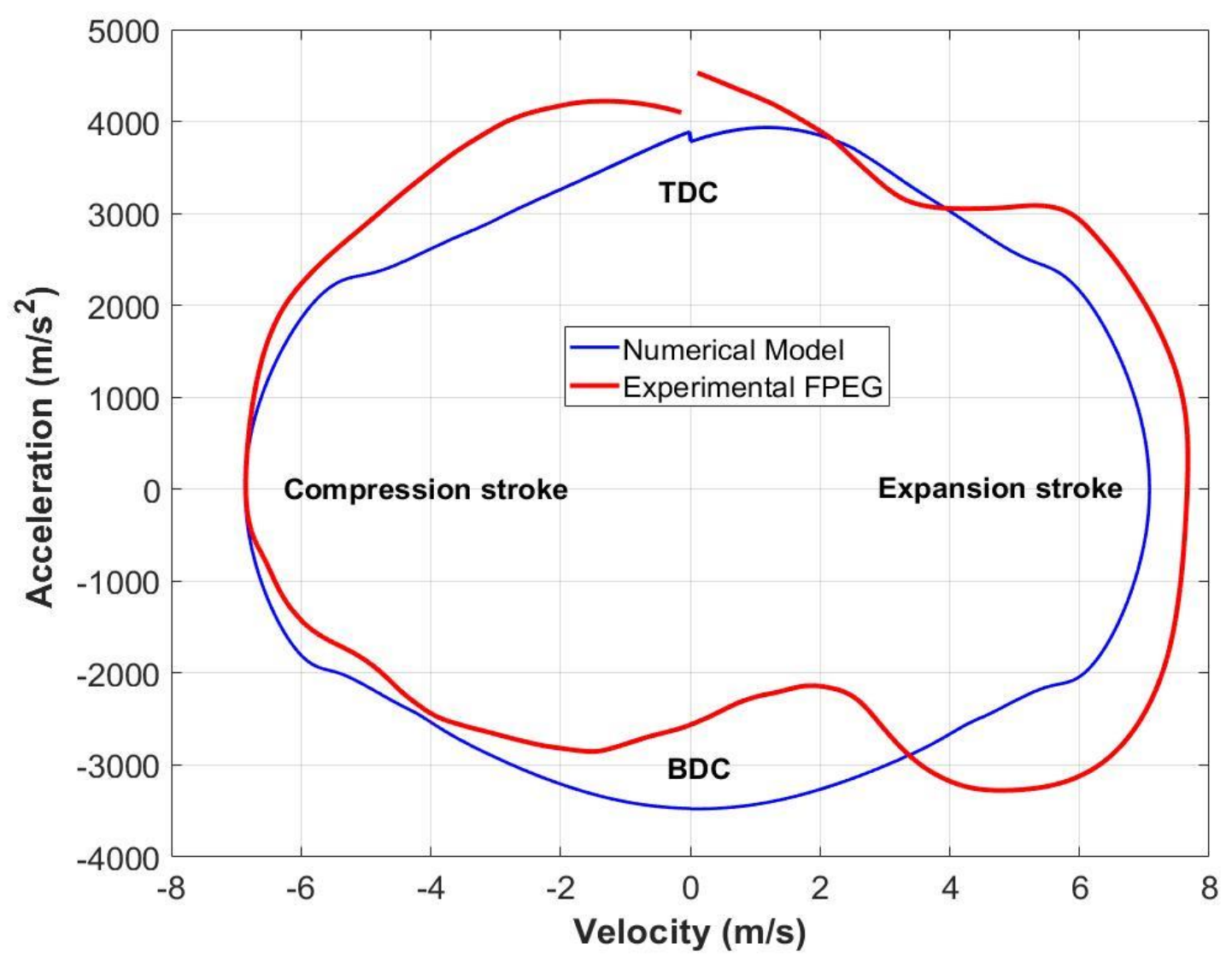

Figure 61: Experimental and modeled dynamics during the FPEG generating process.

The deviations in the model predicted and experimental translator dynamics are shown in Figure 62. From Figure 62, it was evident that the differentiation of the position aggravated the error. With velocity and acceleration calculations, the error was propagated to the first and second orders. Initially, at the dead centers, the translator position deviation was close to $0 \%$. However, the major deviations in the translator displacement were observed in the mid-stroke region. These variations were attributed to two factors. The first factor was the interactions of springs with the external mounts. The second factor was the deviations induced by the mechanical connections. 
From Figure 62, the deviation in the translator displacement was increased from $0 \%$ to $9.1 \%$ from TDC to the mid-stroke. Later, the deviation was reduced back to zero at BDC. On average, the position was deviated by $4.0 \%$ from a cycle-to-cycle basis. From Figure 62 , the deviations in the velocity and acceleration were doubled and quadrupled when compared to the displacement deviation. On an average cycle-to-cycle basis, the deviations in the velocity and acceleration were found to be $9.9 \%$ and $16.5 \%$.

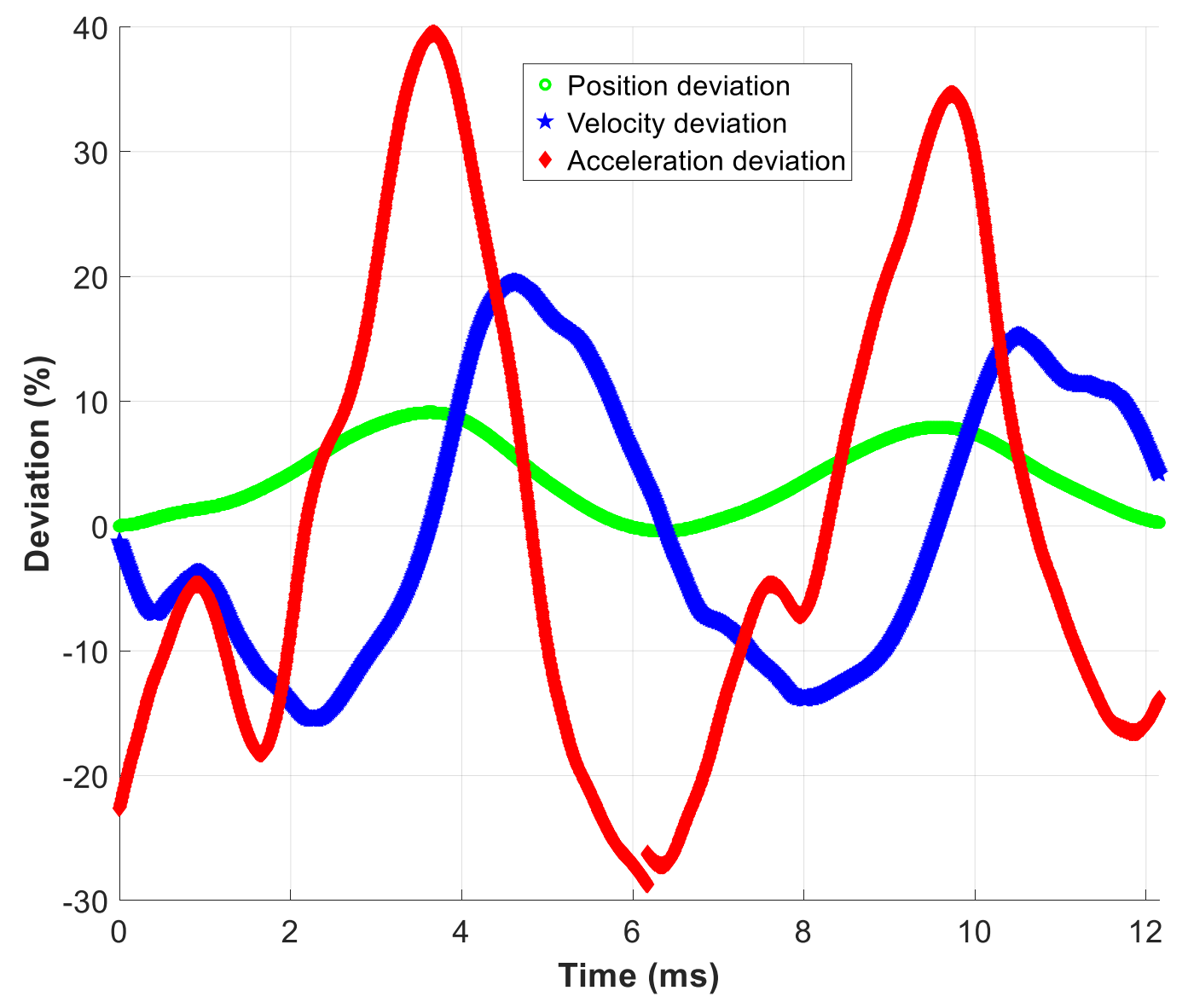

Figure 62: Deviation in the model predicted translator dynamics from experimental dynamics of the FPEG systems.

\subsubsection{Engine characteristics}

The cylinder pressures were modeled in the numerical model by using an open system energy balance equation. The combustion behavior was modeled with a time-based Wiebe heat release function. To predict the combustion characteristics reliably, the combustion submodel outputs 
must mimic the experimental behavior. The main submodels are the HRR, heat transfer rate, and gas exchange functions. Experimentally, the fuel and air mixtures were introduced into the combustion chamber after the crankcase compression. Thus, the use of a constant scavenging pressure in the numerical model was not ideal. As a result, the measured crankcase compression pressures were fed into the numerical model. This was attained by means of one-dimensional lookup tables with respect to the translator displacement. Using the one-dimensional lookup tables, the numerical model obtained the scavenging pressure at each time step. Moreover, the input crankcase compression pressure was also dependent upon the stroke direction. The crankcase compression pressure used in the numerical model is shown in Figure 63.

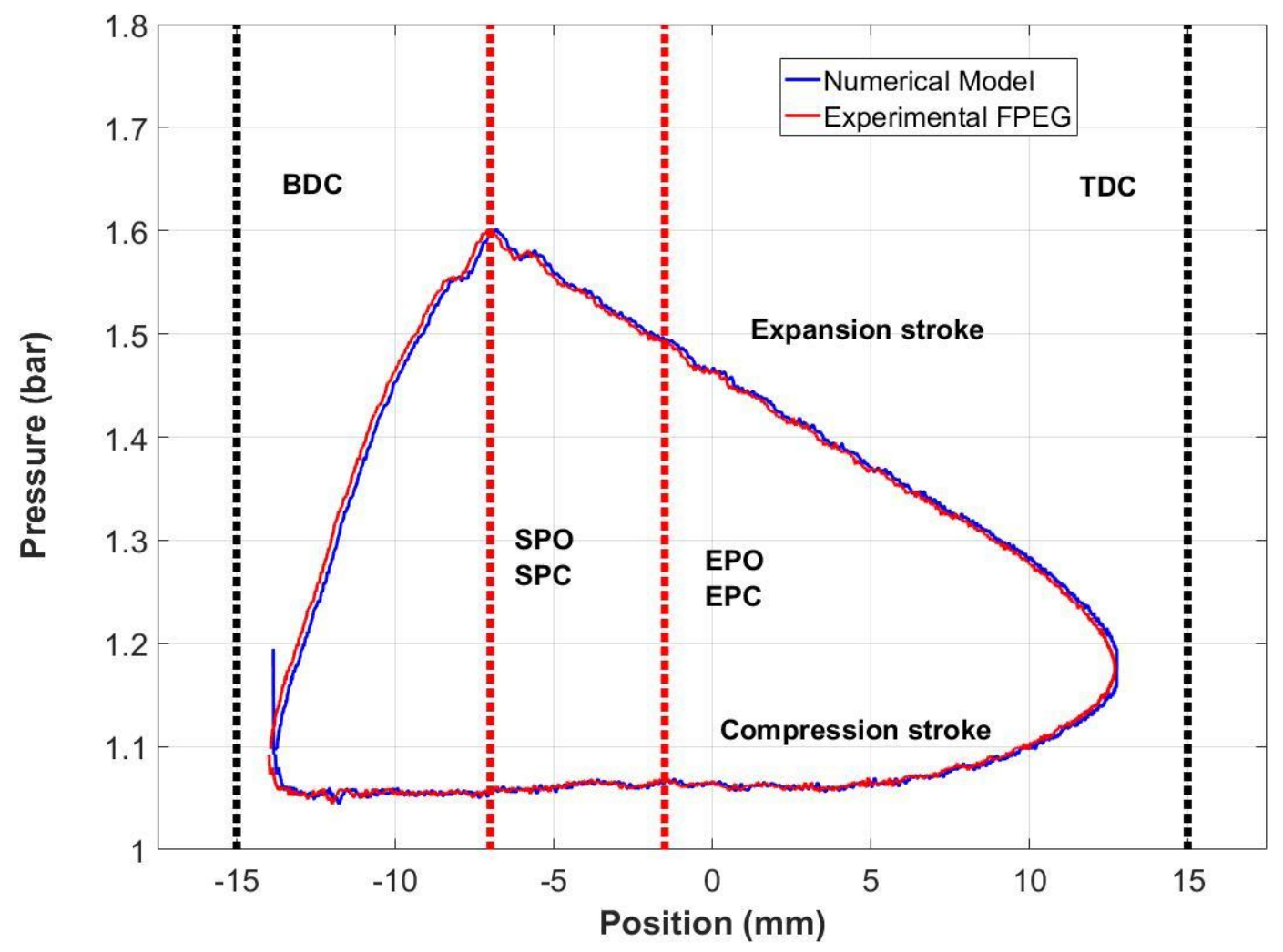

Figure 63: Crankcase pressure provided to the numerical model based on experimental data.

From Figure 63, the numerical model used the same scavenging pressure as that of experimental crankcase compression pressure. The next step in the validation process was the input of the ignition timing. This ignition timing initiated the heat release process in the numerical model. 
Moreover, there was no delay between ignition activation and HRR initiation. The ignition timing in the experimental FPEG system was defined in terms of time after BDC.

The next step in the validation process was simulating the experimental combustion process. This was attained by curve fitting the experimental MFB profile for the time-based Wiebe function. The procedure estimated the form factors and combustion duration. The experimental MFB was calculated based on the experimental cylinder pressure. The cylinder pressures for 750 cycles were averaged to calculate the experimental MFB profile. The procedure for the experimental MFB calculation was described in Section 2.2.4. The experimental and numerically modeled MFB profiles are shown in Figure 64. Due to the combustion instability and cycle-to-cycle variations, the calculated experimental MFB was uneven. From Figure 64, the modeled MFB followed the experimental FPEG MFB profile. Based on the curve fitting techniques, the form factors were found to be 5.8 and 3.2. Moreover, the combustion duration was found to be $2.3 \mathrm{~ms}$. Finally, the combustion efficiency was provided to the numerical model. This value was calculated based on the same CSE developed by Darzi et al. [117].

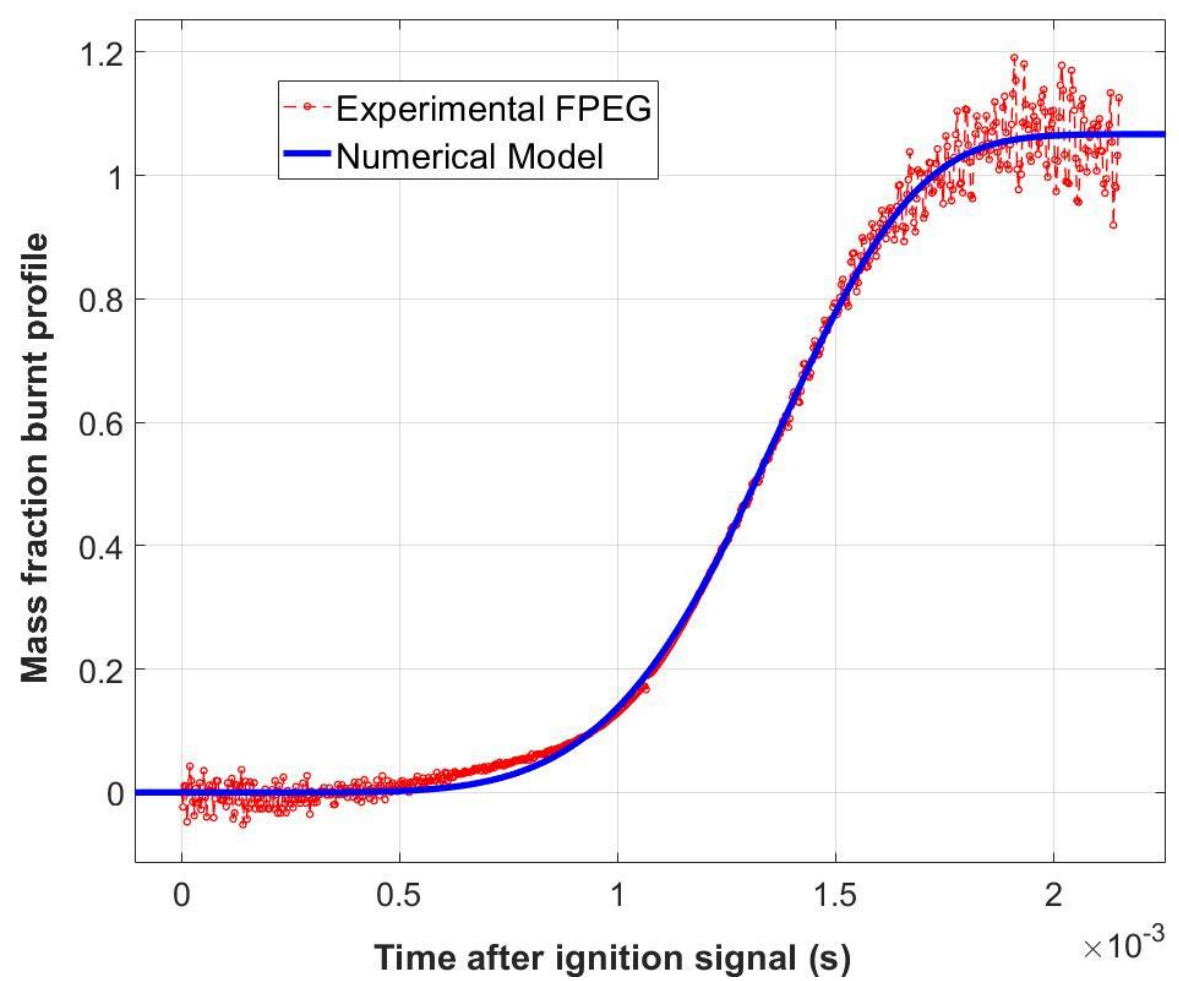

Figure 64: MFB profile estimated by curve fitting techniques in MATLAB®. 
The cylinder pressure plotted against the stroke length is shown in Figure 65. The distribution in Figure 65 was divided to three regions as mentioned in Chapter 2. From Figure 65, it was evident that the numerical model predicted the cylinder pressure reliably. The numerical model predicted the cylinder pressure with a $93.3 \%$ accuracy. Especially, in the expansion process, the modeled pressure followed the same experimental pressure path. This was because of the proper estimation of the HRR process. In other words, the correct Wiebe form factors and combustion duration properly estimated the HRR process. These parameters used in the numerical model were obtained from the experimental HRR. The experimental HRR was predicted using the experimental pressures. The variations in the in-cylinder pressures were observed mainly at the end of the compression stroke. The main reason for the variations between the modeled and experimental cylinder pressures was due to the variation in the dynamics.

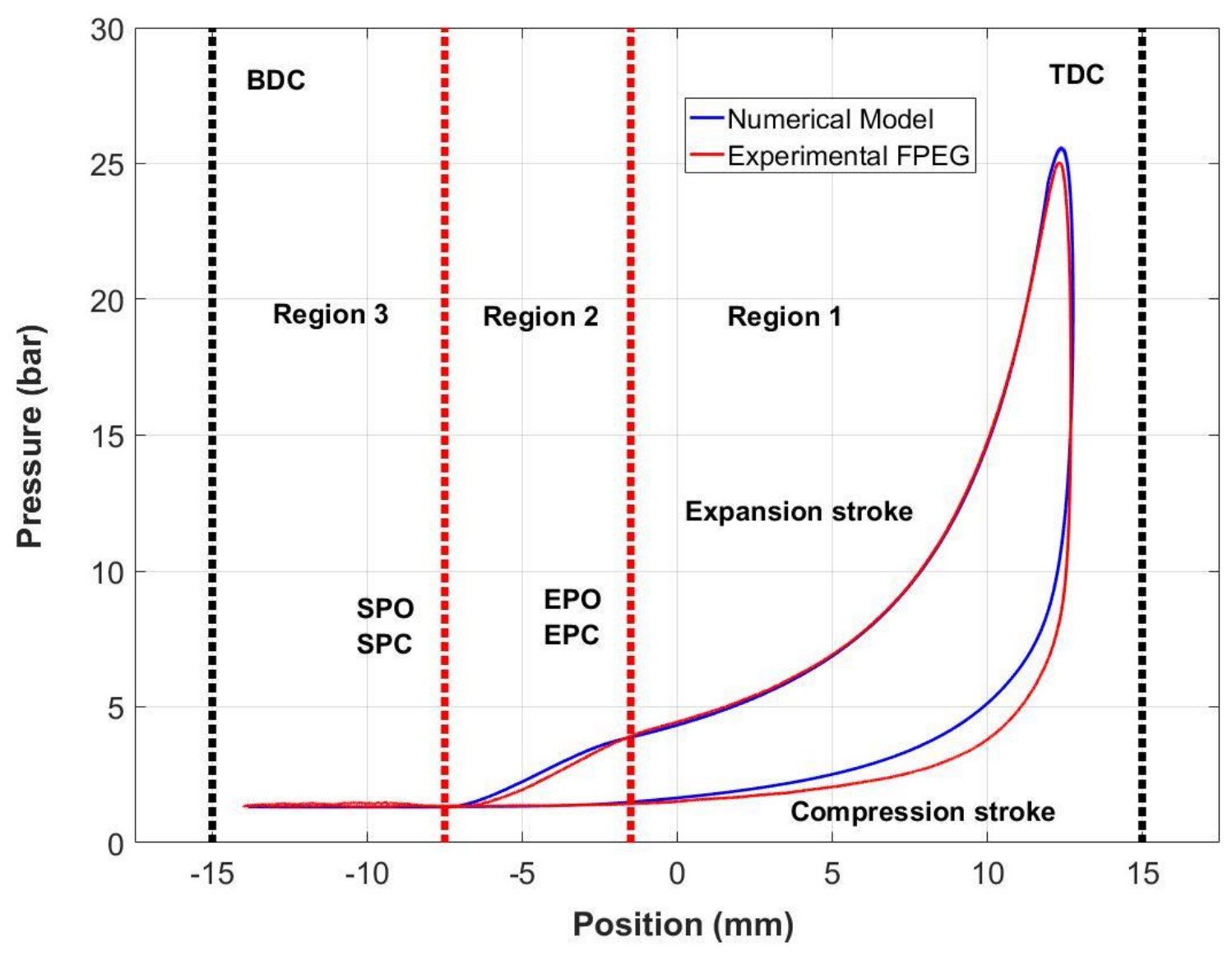

Figure 65: In-cylinder pressure distribution predicted by numerical model \& experiments. 
The other variations were also observed in Region 2. In Region 2, the experimental cylinder pressure was reduced at a much faster rate than the modeled FPEG system. This was due to the variations in the dynamics profile. Moreover, the changes in the volume were higher in the experimental case than the modeled system. The faster change in the cylinder volume reduced the cylinder pressure in the experimental case. Moreover, the experimental system considered an Original Equipment Manufacturer (OEM) exhaust with a certain throat volume. The gas resonant characteristics in the OEM exhaust was also responsible for the cylinder pressure deviation in Region 2. Finally, the cylinder pressure distribution in Region 3 was the same for both modeled and experimental system. This was because of exporting the actual crankcase compression pressure to the numerical model. The variations in the modeled and experimental cylinder pressure are shown in Figure 66.

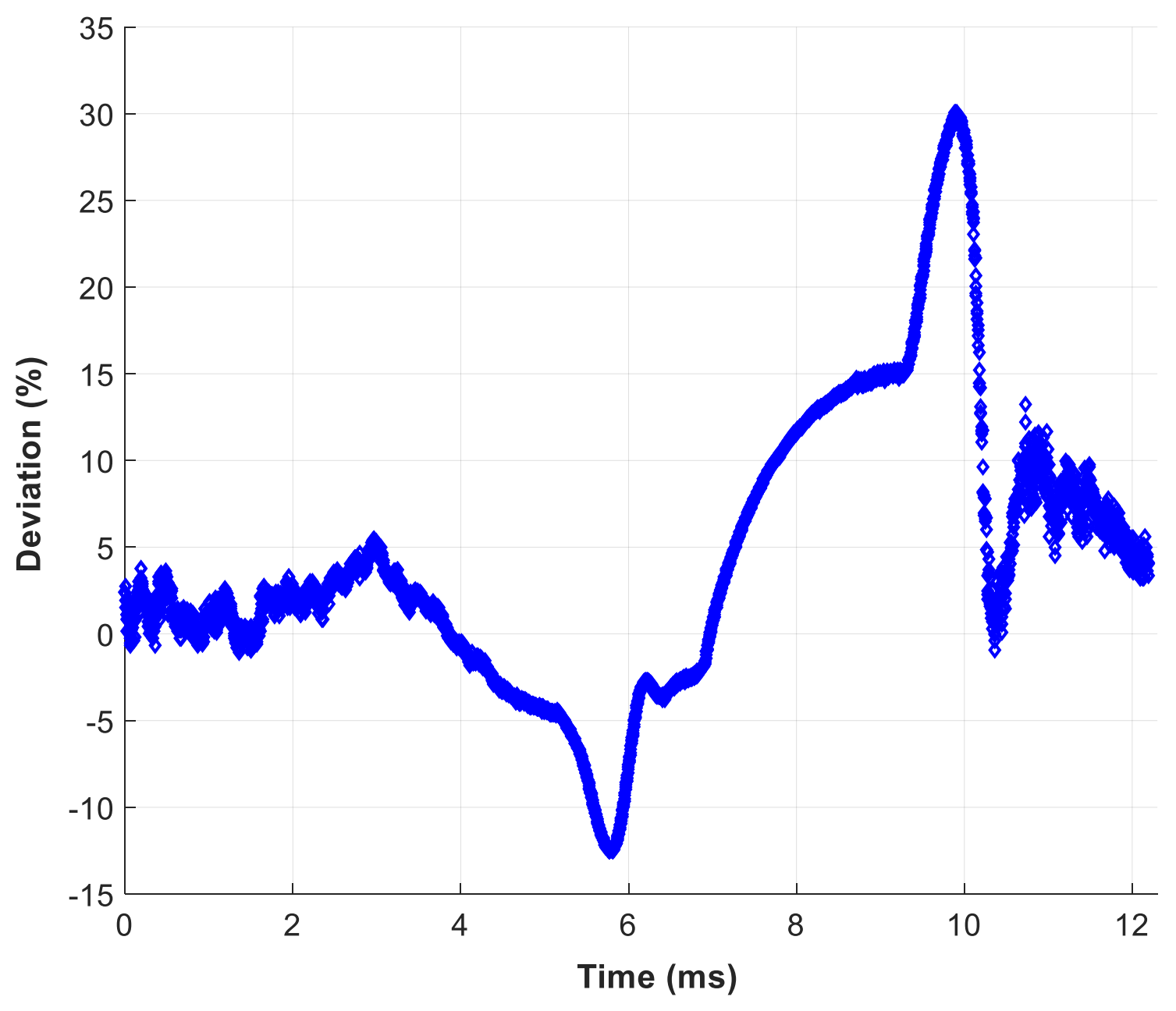

Figure 66: Deviation in the model predicted cylinder pressure from experimentally measured cylinder pressure. 
From Figure 66, initially at BDC, the variations in the experimental and modeled cylinder pressures were less than $5 \%$. This was due to the actual crankcase compression pressure input into the numerical model. Moreover, this was also due to the minor dynamics deviation at the dead centers. The numerical model underpredicted the cylinder pressure in the compression stroke. This underprediction was represented by the negative deviation in the cylinder pressure in Figure 66. Near TDC, the cylinder pressure variations were again less than $5 \%$. However, the major pressure deviations were observed at the time of EPO in the expansion stroke. As shown in Figure 66, the numerical model overpredicted the cylinder pressure in that region by $22.5 \%$. This was due to the variations in the dynamics and OEM exhaust pressure characteristics. However, the variations were reduced from $30 \%$ to less than $10 \%$ at the SPO. On a whole, the engine submodel predicted the cylinder pressure with an $88.3 \%$ accuracy. More specifically, the accuracies were found to be $91.9 \%, 77.5 \%$ and $95.4 \%$ in Regions 1,2 and 3 respectively.

\subsubsection{LEM electrical power output}

The FPEG electrical power output was calculated based on the voltage and current in the windings. In the numerical model, an equivalent circuit model with the load resistance and capacitances was used. Initially, a translational electro-mechanical converter was used in the numerical model. This electro-mechanical converter transformed the velocity to the open-circuit voltage. This was done by using a proportionality constant between the velocity and the voltage. After that, the load resistance and capacitances were provided to the numerical model. These load resistances and capacitances were connected in parallel. The instantaneous load generating characteristics obtained from the numerical model and experiments are presented in Figures 67 and Figure 68.

From Figures 67 and 68, the numerical model predicted the electrical power output with $99.1 \%$ accuracy based on the open-circuit voltage of the experimental FPEG system. The slight variations in the numerical model and experiments were due to changes in the velocity profile. The high accuracy of the LEM model was also due to the minimal electromagnetic forces. According to Figure 60, the electromagnetic forces were around $200 \mathrm{~N}$. This value corresponded to $2.5 \%$ of the peak spring force. 

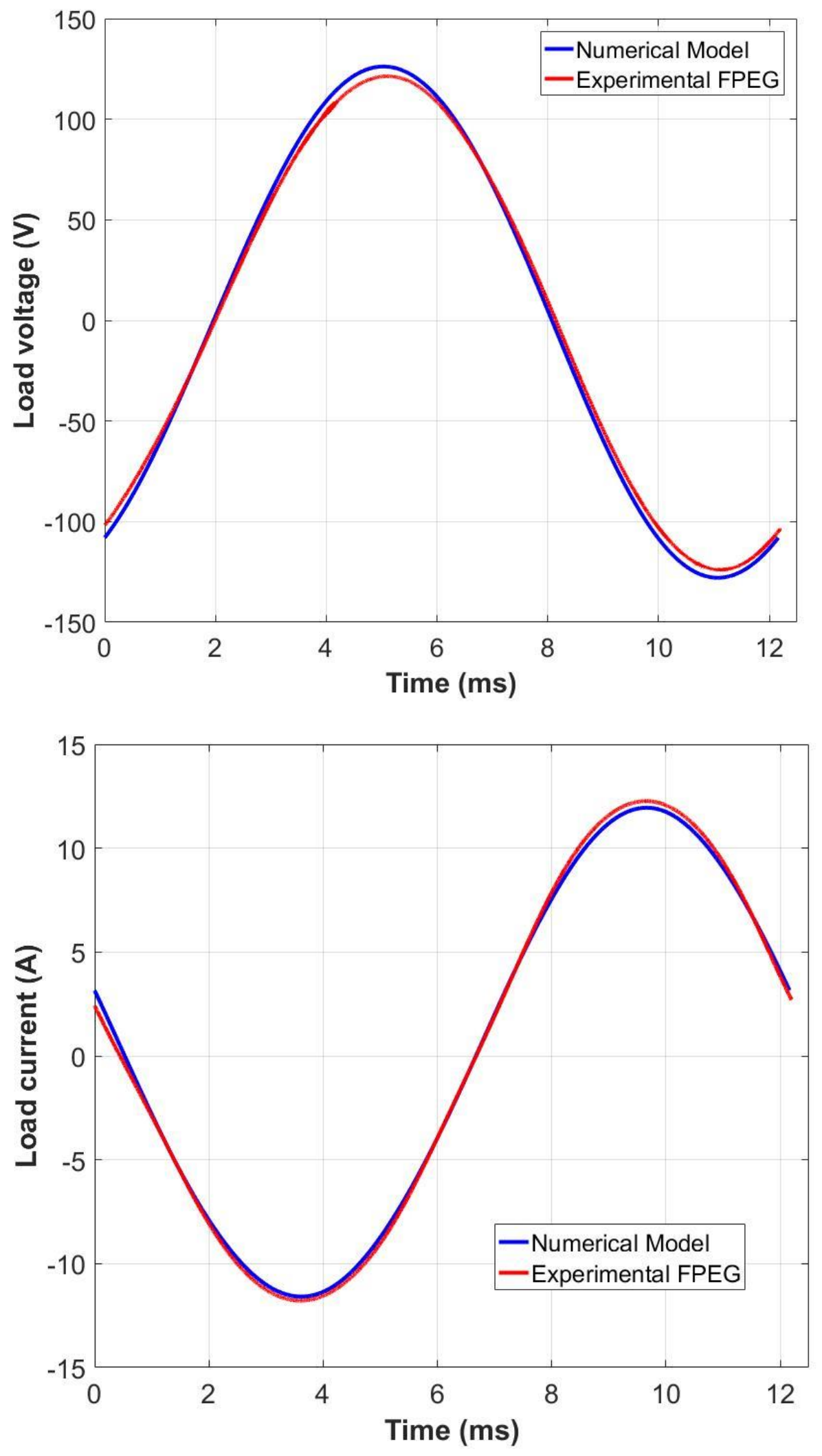

Figure 67: LEM voltages and currents predicted by numerical model \& experiments. 


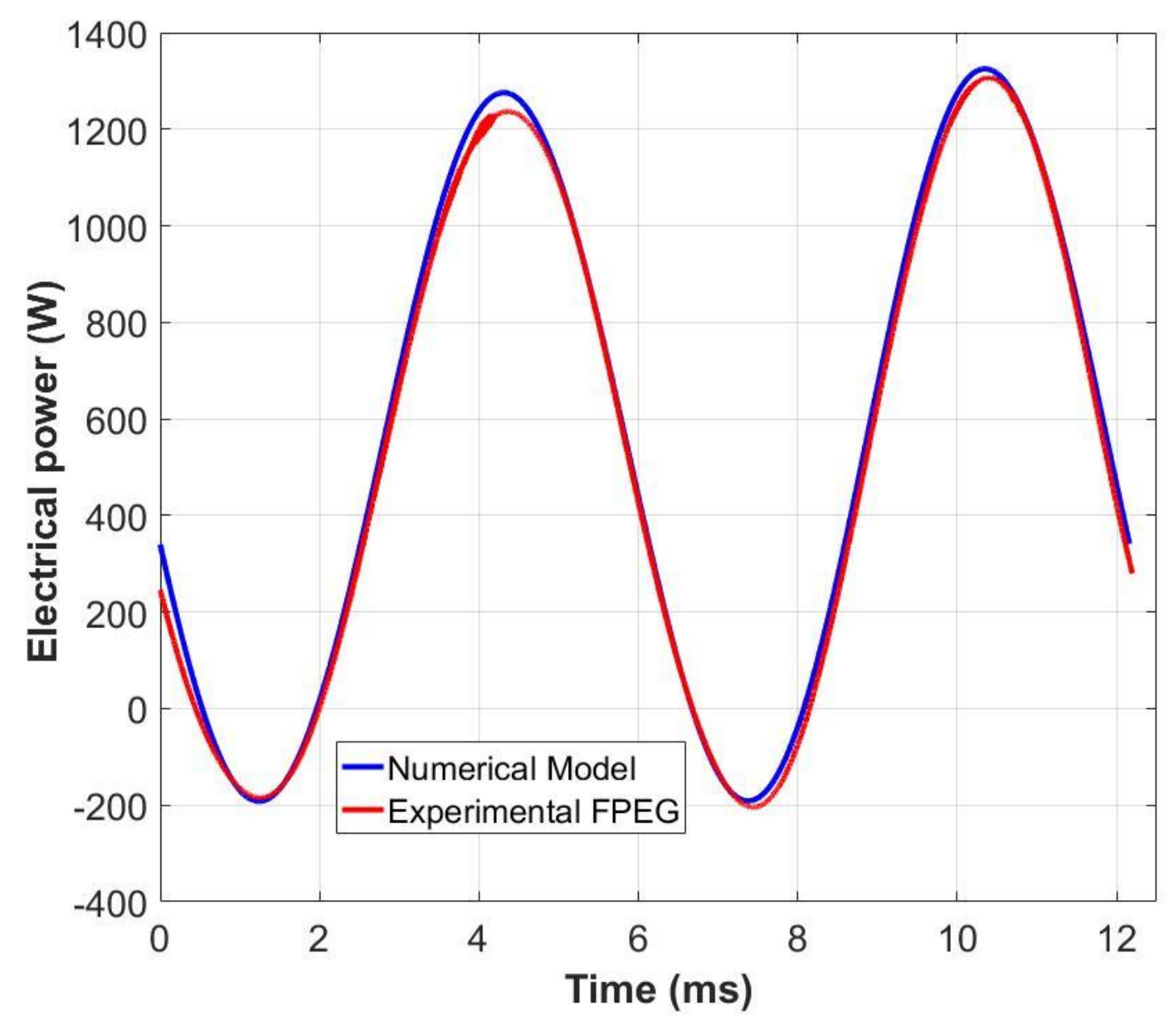

Figure 68: Electrical power output predicted by numerical model \& experiments.

\subsubsection{Energy distribution}

The first law of thermodynamics was used to calculate the energy distribution of the FPEG system. The corresponding mathematical equations were presented in Section 2.4. The pie chart representing fuel energy distribution is shown in Figure 69. Based on the first law of thermodynamics, the numerical model predicted the indicated and FPEG system efficiencies of $13.5 \%$ and $7.3 \%$. Experimentally, they were found to be $13.8 \%$ and $7.9 \%$. Of all the losses, the fuel slip (35\%), exhaust heat (29\%), and heat transfer (21\%) together accounted for $85 \%$ of fuel energy losses. Of these, the fuel slip contributed the most. The rest $15 \%$ was accounted for LEM resistive (4\%), friction (2\%), and combustion losses (2\%) respectively. 


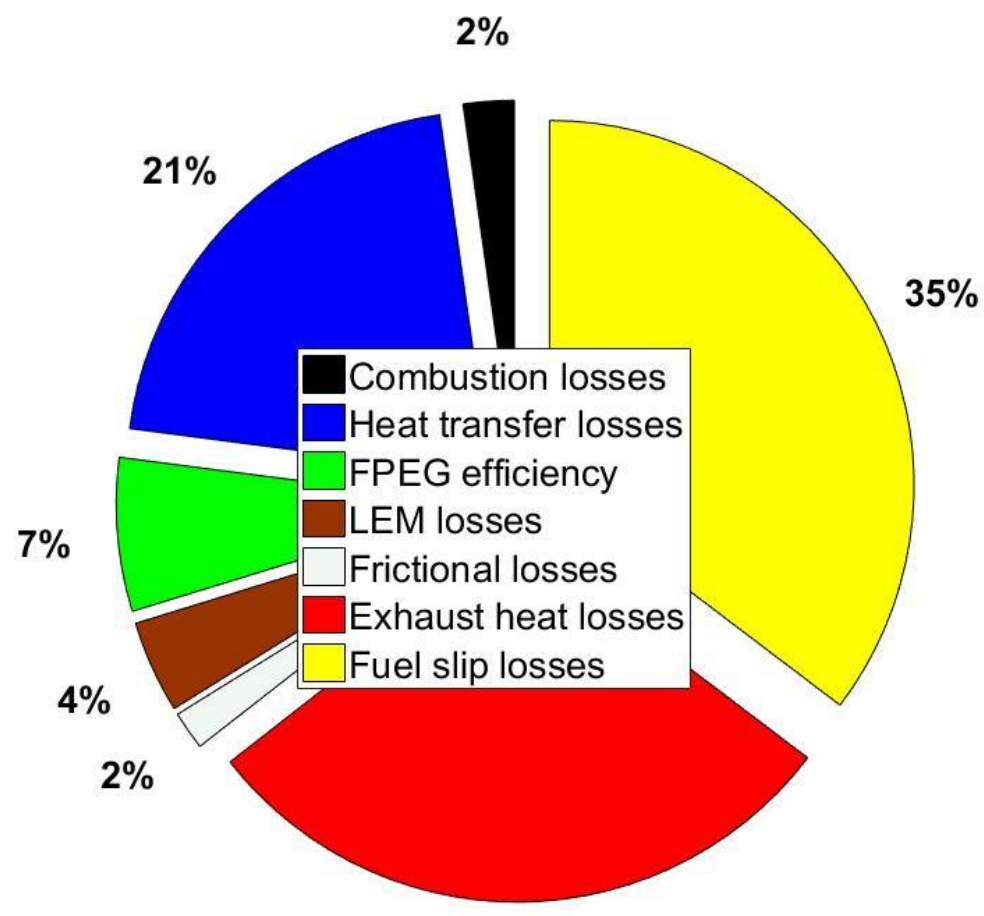

$29 \%$

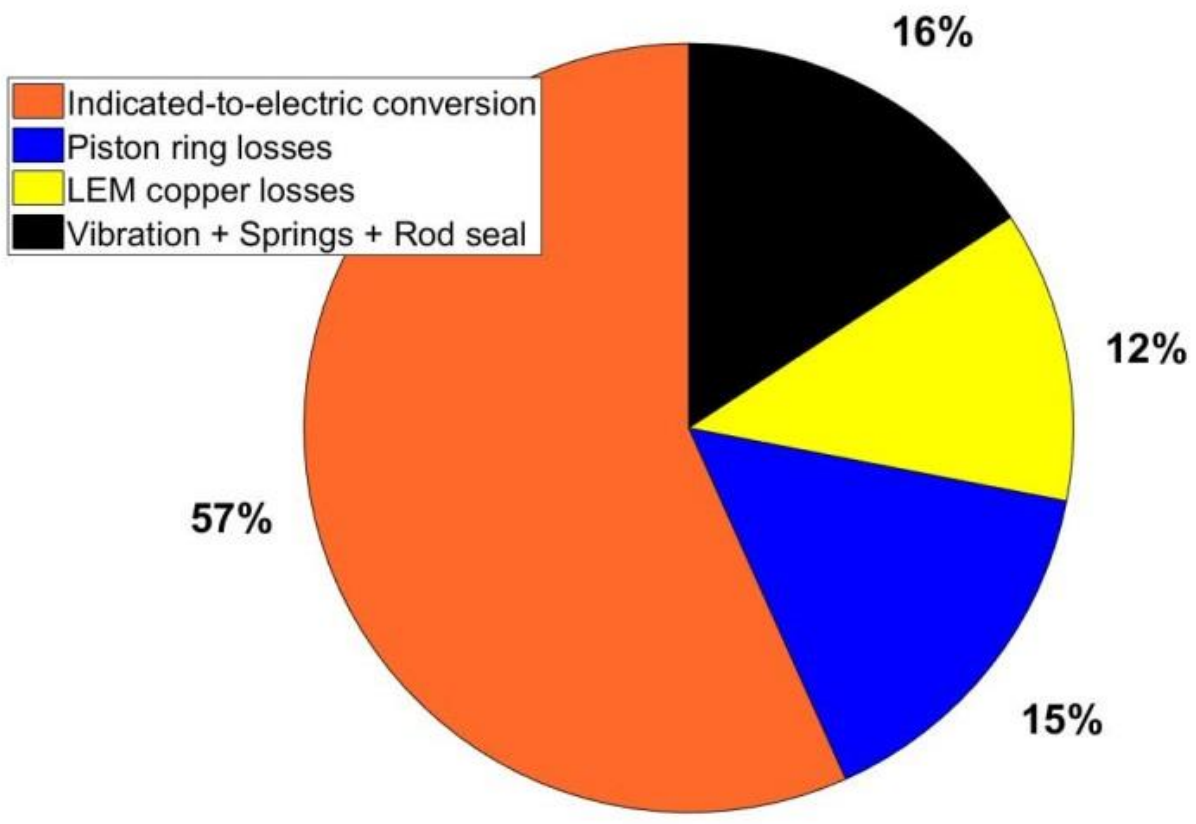

Figure 69: Fuel energy distribution and indicated to electric conversion distributions of FPEG systems. 
Also, in Figure 69, the indicated to electrical distribution for an experimental FPEG system was also presented. Based on the 800 combustion cycles, the engine indicated power was found to be $972.5 \mathrm{~W}$. Of that $972.5 \mathrm{~W}$ of indicated power, $550 \mathrm{~W}$ or $56.6 \%$ was converted into electrical power. The rest of the indicated power was lost to overcome the LEM resistive, piston ring, vibration, and spring losses. The vibration was dissipated into the mounting plate through the external mounts. Based on the calculations, the rest of the losses accounted for $422.5 \mathrm{~W}$ or $43.4 \%$ of indicated power. Based on the current and resistance in the coils, the calculations for the LEM resistive losses were made. The resistive losses were calculated by the product of square of current and resistance in the windings. These resistive losses consumed $114 \mathrm{~W}$ or $11.7 \%$ of indicated power. This value corresponded to an LEM efficiency of $87.9 \%$. Using the piston ring frictional model, the piston ring consumed $147 \mathrm{~W}$ or $15.1 \%$ of indicated power. The rest of the losses were considered as the unaccounted losses. The unaccounted losses in this dissertation were termed as the vibration, spring, and rod seal losses. These losses were calculated by subtracting the electrical power, LEM resistive losses, and piston ring frictional losses from the indicated power. The unaccounted losses were found to be $161.5 \mathrm{~W}$ or $16.6 \%$ of indicated power.

\subsection{Conversion to direct injection}

The major step in improving the FPEG system efficiency was the conversion from fumigation to direct-injected operation. The major advantage of this conversion was increased fuel efficiency and indicated power. Moreover, it also reduced scavenging fuel loss. In a direct-injected operation, a Mercury Optimax gas injector was selected as a fuel injector. These Optimax air injectors were developed initially to operate in marine two-stroke engines. Moreover, they were designed for speeds ranging from 4750-5750 Revolutions Per Minute (RPM). In terms of frequency, this value corresponded to $80 \mathrm{~Hz}$ to $95 \mathrm{~Hz}$. Since the experimental FPEG frequency was in this range, the Optimax injector was selected. The conversion of the FPEG system from fumigation to direct injection enabled stratified lean burn combustion. With the port-injected CSE as a reference, a direct injection operation improved the indicated efficiency and power by $70 \%$ and $25 \%$ [117].

During the stable generating process, the start of injection was fixed at BDC. Moreover, the BDC location changes from cycle-to-cycle. For example, the BDC locations were different for cycles with $26 \mathrm{~mm}$ and $28 \mathrm{~mm}$ stroke length. With the cycle-to-cycle variations, the start of injection was 
either retarded or advanced in the stroke. This was because the ports were at the fixed location even though there were cycle-to-cycle variations. In other words, the start of injection was not optimized for the fixed port locations. Further, the pulse width was varied between $2.5 \mathrm{~ms}$ to 3.5 $\mathrm{ms}$ for a range of 800 cycles. The low pressure compressed natural gas was supplied to the injector from the gas cylinder. The natural gas was maintained at a pressure of 4 bar. The supply lines were connected from the gas cylinder to the fuel injector. A mass flow controller was placed between the gas cylinder and a fuel injector. A buffer volume was placed in-between the mass flow controller and the fuel injector. This buffer volume reduced the pressure fluctuations due to the fuel injector operation. Similar to the fumigated operation, the direct-injected FPEG system was made to run without any resonant exhaust and intake systems. The intake air of 113.3 SLPM was delivered to the combustion chamber through the crankcase. The FPEG system delivery ratio was found to $68 \%$ when an effective stroke of $30 \mathrm{~mm}$ was considered. The ignition timing was found to be in-between $5.4 \mathrm{~ms}$ to $5.7 \mathrm{~ms}$ after BDC.

Preliminary experiments were carried on the FPEG system with the direct-injected operation. The preliminary direct-injected system generated $350 \mathrm{~W}$ of electricity. The main objective of the preliminary experimental data was to show the unstable nature of the directed-injected FPEG system without an external controller. This unstable nature of the FPEG system was attributed to multiple causes. One cause was due to the combustion instability induced by the varying stroke length. With the variable stroke length every cycle, the injector and ignition parameters were not optimized. As a result, the fuel was injected and ignited at the wrong locations within the cycle. Moreover, with cycle-to-cycle variations, the gas exchange durations were also changed. In other words, the opening and closing timings of the ports were not the same. These port operations changed the amount of fuel and air mixture trapped in the cylinder. In addition to these, the lack of resonant intake and exhaust systems was also responsible for the cycle-to-cycle variations. Figure 70 illustrated the variation in the dynamics for a direct-injected FPEG system for 50 consecutive cycles. Based on Figure 70, the FPEG system exhibited variable stroke length due to variations in the combustion and mixture trapping characteristics. Finally, the cycle-to-cycle variations were due to the unstable combustion forces and cylinder fuel flow. 

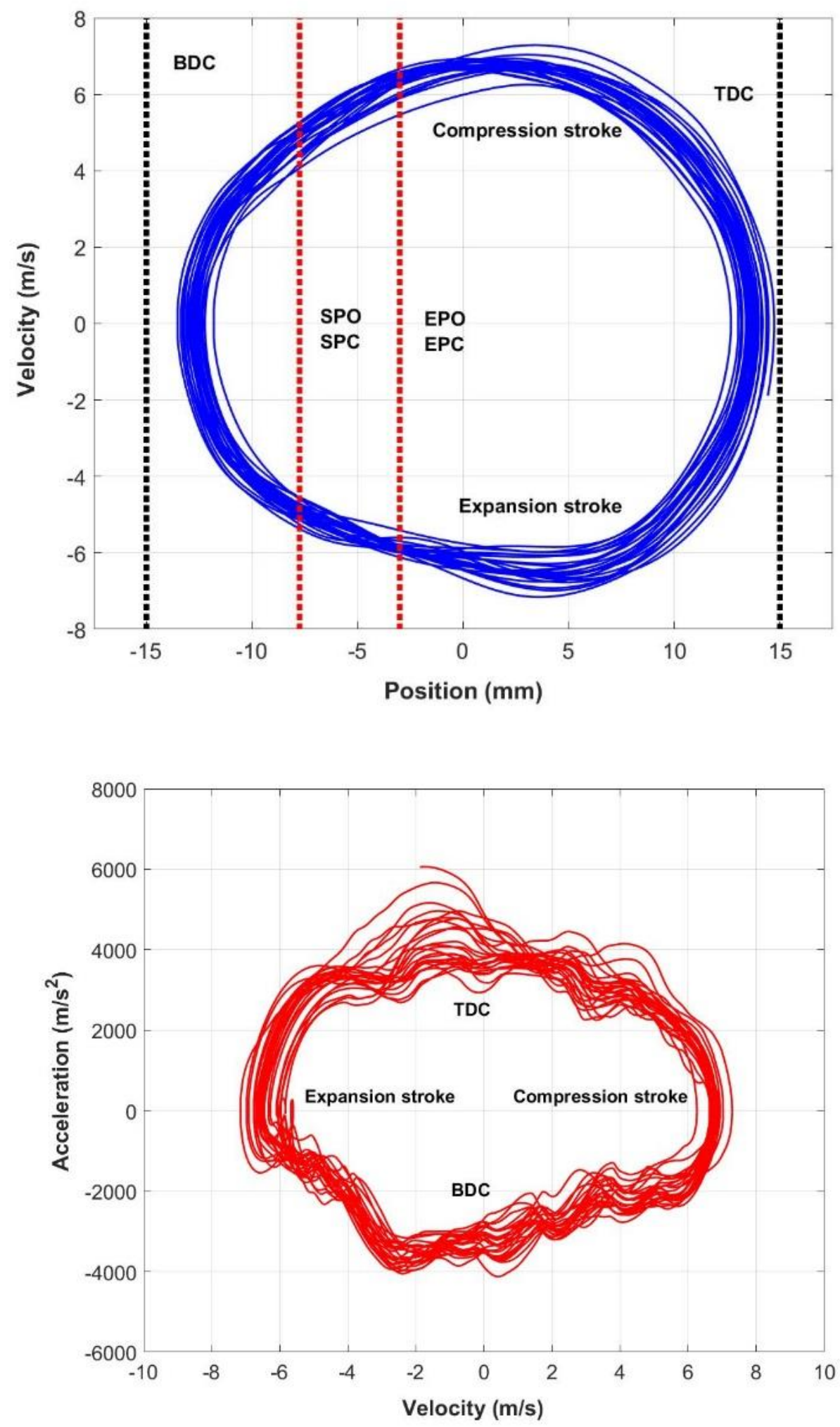

Figure 70: Translator dynamics of a direct-injected FPEG system. 
Secondly, the ignition cutoff was also responsible for the unstable FPEG operation. With the ignition cutoff, the translator displacement and cylinder pressures were reduced in the upcoming cycles. These displacement variations induced by the ignition cutoff further reduced the mixture delivered into the cylinder in the upcoming cycles. In summary, the direct-injected FPEG behavior was influenced by history effects, so that cycle-to-cycle behavior was not repeatable.

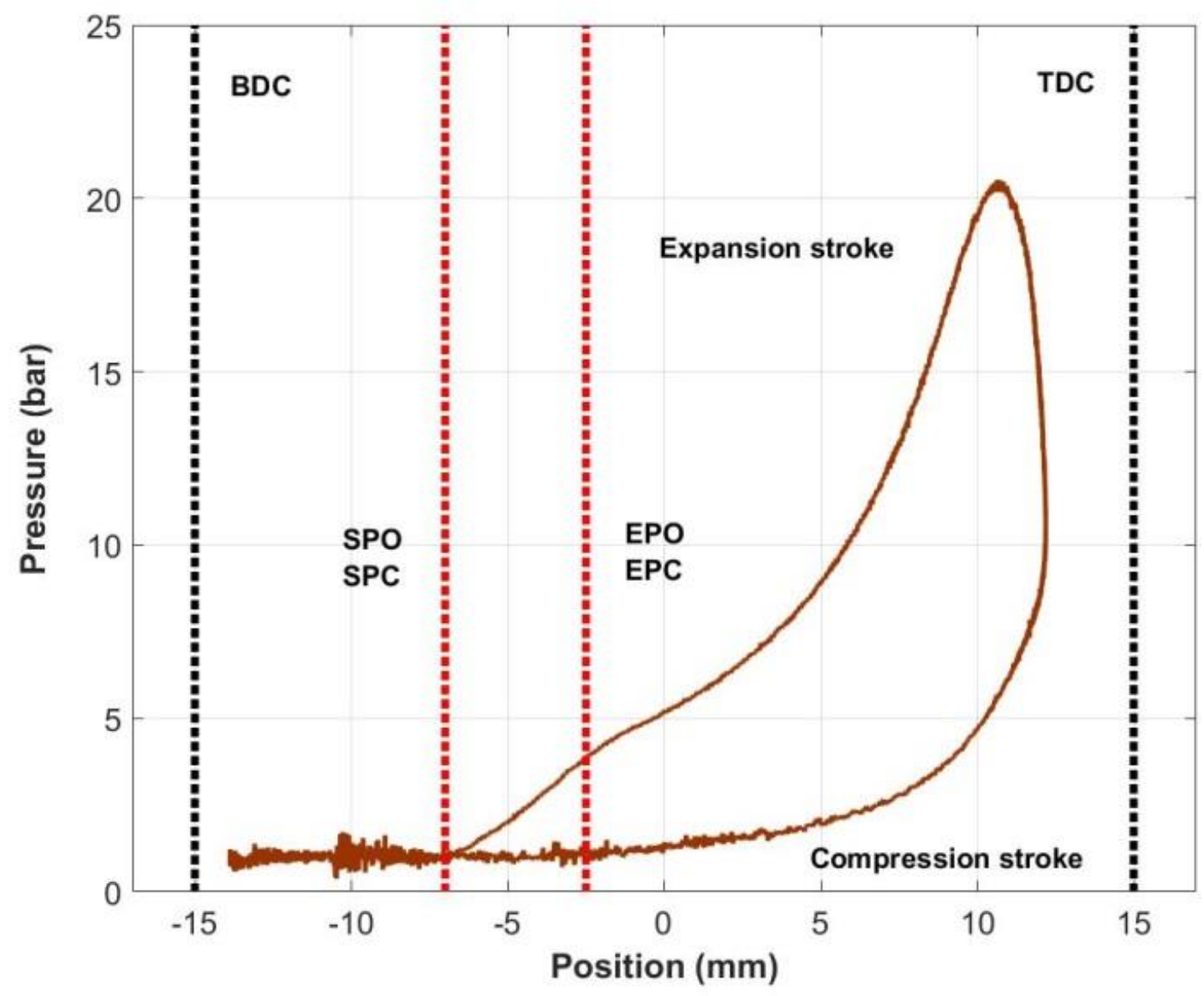

Figure 71: Cylinder pressure distribution of a direct-injected experimental FPEG system.

Figure 71 shows the average pressure distribution of the direct-injected FPEG. A total of 600 cycles with the indicated power greater than $400 \mathrm{~W}$ were considered. This was to eliminate the cycles after the ignition cutoff. When compared to Figure 65 , the peak pressure occurred much later in the expansion stroke. This delayed peak pressure was mainly because of two causes. 
The first cause was the non-optimized ignition timing. The second cause was due to the amount of fuel delivered into the system. With the ignition cutoff, the trapped mixture inside the cylinder was either too rich or too lean. This trapped mixture reduced the combustion forces. The combustion forces further reduced the displacement and trapped compression ratio in the next cycles. These variations further reduced the indicated results. All these factors resulted in the unstable operation of the direct-injected FPEG prototype.

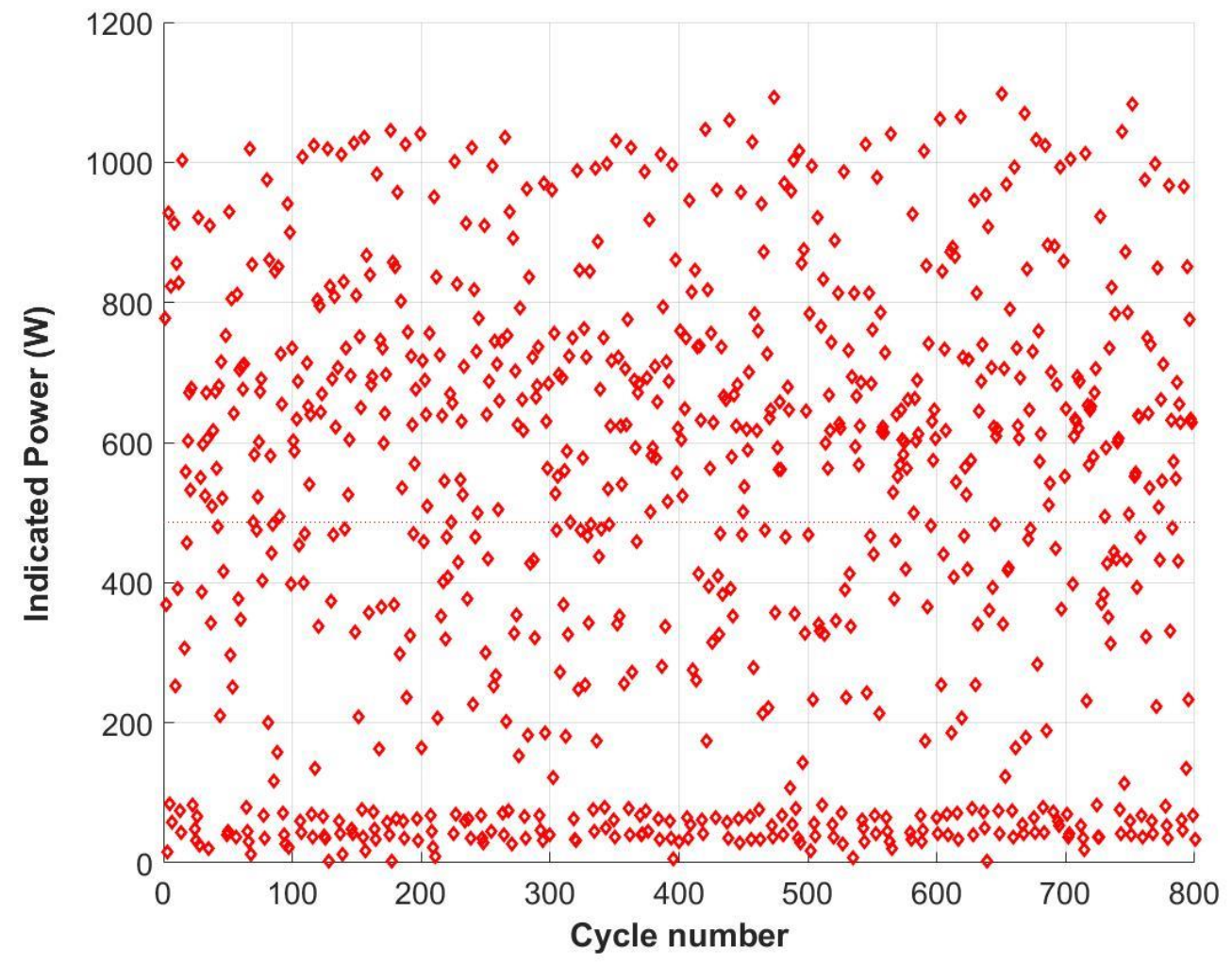

Figure 72: Cycle-to-cycle variations in the indicated power of a direct-injected FPEG system.

The cycle-to-cycle variations in the indicated power during the direct-injected operation is shown in Figure 72. Over a span of 800 cycles, the indicated power was varied from $0 \mathrm{~W}$ to $1050 \mathrm{~W}$. The cycles with the indicated power below $100 \mathrm{~W}$ were the cycles with the ignition cutoff. Even though the ignition was turned off in a particular cycle, the LEM still generated the power output from the system. For example, for the cycle with an indicated power of $100 \mathrm{~W}$, the LEM still generated 350 W of electrical power output. Since the electrical power was higher than the indicated power, the cylinder pressure, displacement, and velocity at mid-stroke were reduced on the few following cycles. Therefore, it is essential to manage the energy in the FPEG system. This can be done by 
matching the engine output to the generator demand. In other words, an energy balance control algorithm is required for the stable FPEG operation. This energy balance control algorithm is devised and explained in Chapter 5 of this document.

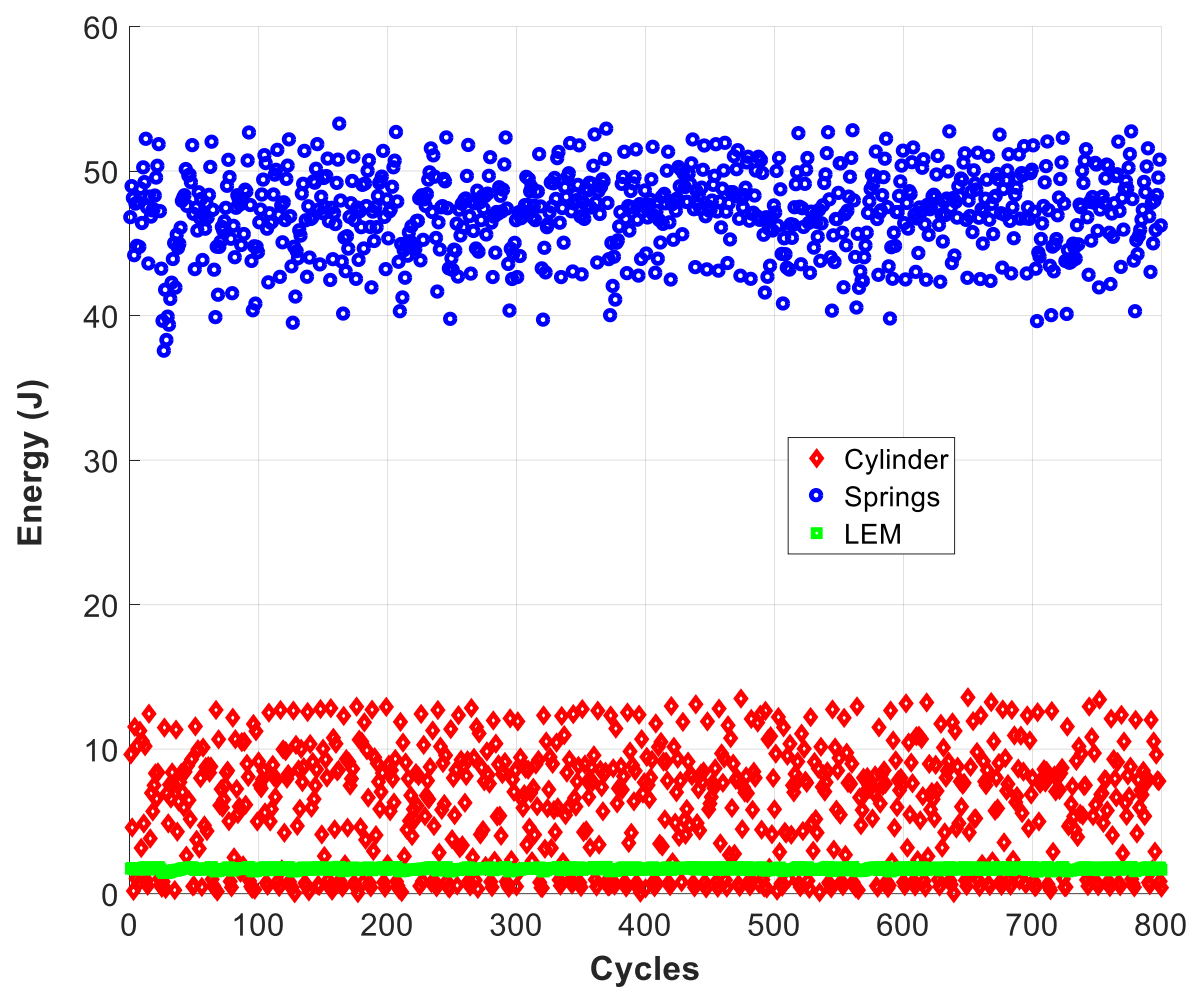

Figure 73: Variations in the energy stored in springs, LEM and energy generated by combustion in a direct-injected FPEG system.

Figure 73 shows the variations in the amount of energy stored in the springs, LEM, and the amount of combustion energy generated by the engine. These energy data were presented for a span of 800 cycles in a direct-injected FPEG operation. From Figure 73, the LEM energy almost stayed constant at $1.7 \mathrm{~J}$ for all the 800 cycles. This was due to the constant electromagnetic force and electrical power output from the generator. The variations were observed mainly in the energy stored in the springs and the combustion energy generated by the engine. Based on Figure 73, the combustion energy generated by the engine was varied from $0 \mathrm{~J}$ to $12 \mathrm{~J}$. Due to the combustion instabilities, the energy stored in the springs was varied from $40 \mathrm{~J}$ to $52 \mathrm{~J}$. In other words, a high interdependency was observed between the combustion instabilities and mechanical spring forces. 


\section{SENSITIVITY ANALYSIS \& PARAMETRIC STUDIES}

In Chapter 2, the MATLAB®/Simulink based numerical model was developed. This numerical model was validated using the experimental FPEG data in Chapter 3. This chapter explored the design space of the numerical model by using parametric and sensitivity analysis studies. The results were used to understand the effect of input parameters on the model outputs. Moreover, the sensitivity analysis was used to understand to rank the model inputs. The inputs were ranked in the order of their dominance on FPEG power and efficiency. The conclusions from these studies supported the interdependence of model inputs in defining the model outputs. Moreover, they provided the basis for the optimization of the experimental FPEG system.

In this dissertation, only main geometric, design, and operating parameters were considered. This was due to the time required for one model evaluation. For example, the change in the stroke length changed the port locations, port operations, and flow characteristics. These variations further influenced various submodel outputs within the engine cylinder. Similarly, the design parameters like spring stiffness and moving mass also influenced FPEG model outputs. This increase was due to an increase in the frequency and power density. Increasing the spring stiffness increased the FPEG electrical power. However, an increase in the moving mass reduced the electrical power. This example implied the strong interdependence of model inputs in determining the FPEG model outputs. Considering this strong interdependency, the major objective of this Chapter was to identify the major input variables. Finally, these inputs were ranked in order of dominance with respect to FPEG performance.

In the numerical model, a substantial effort was made to have a high level of sophistication. For example, the LEM submodel was developed based on equivalent circuit parameters. However, LEM characteristics depended upon multiple factors like air gap, magnet thickness, number of coil windings, design, and magnet parameters. The optimized LEM design can be obtained through finite element analysis methods. This finite element method was beyond the scope of this dissertation. However, the MATLAB®/Simulink LEM equivalent circuit model can be adapted for a finite element model. In this case, an S-function can be used to connect the finite element model to the Simulink model. Similarly, the stiff springs were assumed as linear with a constant damping coefficient. However, these stiff springs can be modeled with and without any losses due to mount vibrations. In summary, the numerical model can be expanded to use different component submodels. 
For any complex models, the output uncertainty can be distributed to the different model input uncertainties. This process of distributing the output uncertainties is called sensitivity analysis. Moreover, each and every model input is associated with quantitative values. These values are known as sensitivity indexes. There are two different approaches to carry out the sensitivity analysis. They are local sensitivity and global sensitivity analysis. In this dissertation, both methods were used. Initially, a local based sensitivity approach was utilized. This approach allowed to reduce the number of various model inputs. In other words, the local sensitivity highlighted the main inputs that had a dominant effect on the model outputs. The selected inputs from the local sensitivity study are then used as the basis for the global sensitivity study. Finally, the global sensitivity results ranked the model inputs in the order of their importance.

The local sensitivity analysis is also called as a derivative or numerical approach. This method calculated the sensitivity index based on the partial derivative or Jacobian function. The name local denotes that the Jacobian is calculated at a single value. In this method, only one model input is varied at a time. Therefore, this method is also called as One-At-a-Time (OAT) method. Apart from one input, all other model inputs are fixed. For this method, only a few model evaluations are required. The uncertainty is calculated based on the Jacobian function. Moreover, there are no interactions with other model inputs in the local sensitivity study. As a result, the sensitivity value considered no uncertainties due to the interactions of model inputs.

In this dissertation, for the local sensitivity analysis, a total of 25 parameters was considered. These 25 parameters were divided into three different groups. The first group considered cylinder geometry and flow conditions. This group included the cylinder bore, maximum stroke length, scavenging pressure, exhaust pressure, and intake temperature. The second group considered the port locations and geometrical conditions. The second group parameters were the exhaust port location, exhaust port height, exhaust port width, scavenging port location, and scavenging port width. These port geometries were defined as a percentage of stroke length. Moreover, with the changes in the stroke length, these locations were changed. Therefore, the maximum cylinder stroke length and cylinder operating conditions were maintained the same in this case. In other words, only the port location percentages were changed for the same stroke length.

The third group considered the FPEG system design and operating parameters. This group included the HRR, heat transfer, LEM, and spring operating variables. Especially for the HRR, 
the combustion efficiency, combustion duration, and Wiebe form factors were considered. As mentioned in Chapter 2, the FPEG heat transfer process was modeled based on the Hohenberg heat correlation coefficients. These Hohenberg heat transfer coefficients were also considered for the local sensitivity study. Finally, the LEM efficiency, equivalence ratio, compression ratio, ignition timing, spring stiffness, and moving mass were also considered. Initially, all these 25 model inputs were varied individually around the fundamental values mentioned in Table 2. A total of 15 samples were considered for each input. In other words, a total of 400 model evaluations were performed. A fixed time step of $0.5 \mu$ s was considered for the model. The numerical model was made to run for a maximum time of $10 \mathrm{~s}$. Each model evaluation took 19 minutes to generate the model output.

Next, a global sensitivity study was performed. This study considered the interactions of model inputs. Unlike the local sensitivity approach, the global approach explored a large region of the design space. The global sensitivity approach was implemented by using Monte Carlo techniques. Depending upon the type of models, different methods were used to calculate the sensitivity indices. For a linear model, the sensitivity indices were obtained from Pearson, standard regression, and partial correlation methods [120]. For a non-linear model, the sensitivity indices obtained from Spearman, and partial Spearman regression methods were considered as best choices [120]. A non-linear model refers to the non-linear relationship of model output with one or more model inputs. The current numerical model output followed a non-linear trend. Thus, the Spearman regression methods were considered for calculating the global sensitivity indices in this dissertation.

For the initial global sensitivity study, a total of 7500 numerical samples was considered. These samples were obtained using the Sobol space filling algorithm. Moreover, a uniform probability distribution was considered for a given range of the variable [121]. The results from the local and global sensitivity studies were used to select a group of final 6 model inputs. These 6 model inputs were selected as a basis for the final global sensitivity study. In the final global sensitivity study, a total of 3000 samples was considered. Finally, these 6 model inputs were ranked in the order of their dominance. In summary, the model was evaluated 10500 times for the global sensitivity analysis studies. 


\subsection{Sampling methods}

The important step before starting the sensitivity analysis studies was the sampling of numerical model inputs. This section described the statistical sampling method employed to define to samples of model inputs within the certain range. After defining the samples, a Monte Carlo Simulation approach was used to evaluate the model for the sampled inputs. The results from these evaluations were used to calculate the uncertainty in the model output.

In this investigation, a Sobol's space filling algorithm with a uniform probability distribution was used to generate the samples for model evaluation. The term space filling referred to the filling of the domain space with low discrepancies. The uniform rectangular distribution followed a constant probability. This constant probability density for the LEM efficiency is shown in Figure 74 . This distribution was defined based on the maximum and minimum value of the model inputs. Based on these values, the samples were uniformly and equally sampled. The probability distribution function for the uniform distribution is given in Eq. 1.

$$
\varnothing(x)=\left\{\begin{aligned}
0, & x \notin\{\text { Max }, \text { Min }\} \\
\frac{1}{\operatorname{Max}-\operatorname{Min}}, & x \in\{\operatorname{Max}, \operatorname{Min}\}
\end{aligned}\right.
$$

Later, the Sobol algorithm created the sequences in the region defined based on the number of samples, maximum, and minimum values [121]. Moreover, this algorithm generated low discrepancy, quasi-random sequences when compared to the random number approach. The sequences generated by the Sobol algorithm filled the domain space evenly. The 1500 samples generated by the Sobol space filling algorithm for the scavenging and exhaust pressures are shown in Figure 74. The scavenging pressure referred to the pressure at which the fuel and air mixtures were introduced into the cylinder domain. 

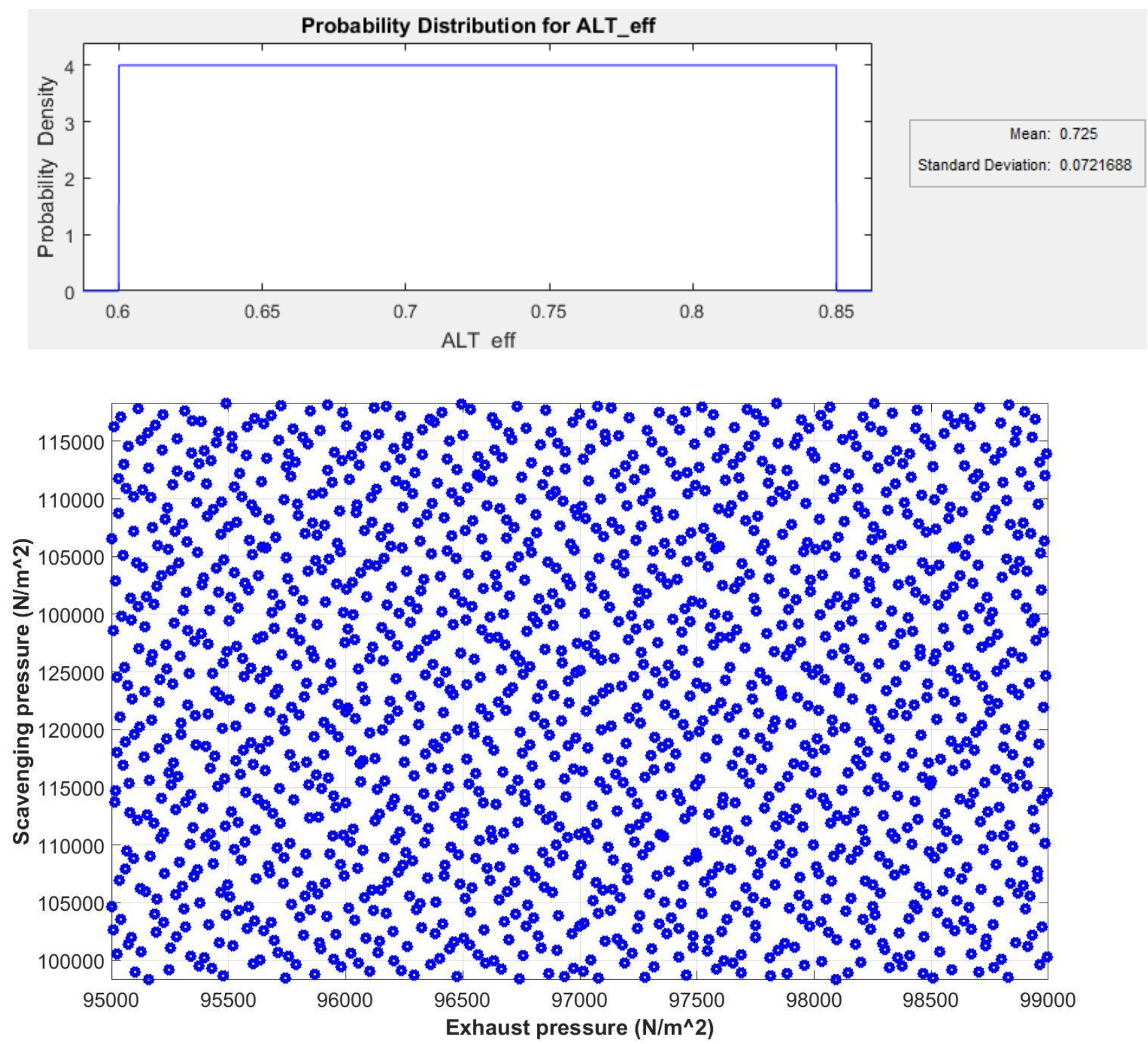

Figure 74: Uniform probability distribution function (top) and samples generated from Sobol alqorithm (bottom).

From Figure 74, the Sobol space filling algorithm generated the low discrepancy and evenly spaced samples. This algorithm was important, especially for the complex models with high computational expenses. This was because the Sobol space filling algorithm reduced the number of samples. In addition to the uniform distribution, other probability distribution functions can also be used. The functions are the normal, gaussian, beta, gamma, and exponential distributions [120]. Similarly, in the place of Sobol algorithm, the random and Latin hypercube sequences can 
also be used. However, the current study was only limited to the uniform probability distribution with Sobol sequences. Moreover, the main reason for considering the uniform sampling was to explore the whole design space. In a normal distribution, the samples were restricted around the mean value of the model input.

\subsection{Model evaluations \& Screening methods}

The numerical model calculated the cost function for every model evaluation. The cost function referred to the output obtained by varying one or more model inputs. The cost function was evaluated for all the ' $\mathrm{N}$ ' samples of model inputs. Each and every sample of the model input was provided to the numerical model as a row vector. For each sample with multiple model inputs, the numerical model provided a single value for the cost function. Similarly, for ' $N$ ' samples, the numerical model provided ' $N$ ' values of the cost function. If multiple outputs were considered, the Simulink $\circledast$ Design optimization toolbox generated a matrix of size $N \times Y$. The term ' $Y$ ' was the number of outputs considered for the cost function. Later, the sensitivity analysis was carried out individually on each column of the cost matrix. The sensitivity analysis studies were performed using multiple screening methods. In this dissertation, the screening methods were Jacobian function and Spearman ranked correlation methods. The corresponding equations and the procedure were described in the upcoming sections. Initially, the local sensitivity analysis was used to identify and segregate the important model inputs. This identification and segregation formed a basis for the global sensitivity study. Finally, the Spearman correlation coefficients were used to rank the inputs in the order of their dominance.

\subsection{One-At-a-Time (OAT) method}

The simplest screening method was the OAT method. In this method, only one model input was varied individually. All other model inputs were maintained fixed. The model inputs were then segregated based on their dominance. The major advantage of this local sensitivity method was the low computational time. Moreover, only a few model evaluations were required to obtain the sensitivity index. However, the major disadvantage was that it failed to consider the interactions between the model inputs. Hence, the sensitivity index was qualitative rather than quantitative. In other words, this method failed to provide information about how much each model input was more significant than other model inputs. Finally, the sensitivity index was calculated. This index 
was calculated using the partial derivative or the Jacobian function. The Jacobian function of the output with respect to the input is shown in Eq. 2.

$$
\text { Local Sensitivity Index }(L S I)=\frac{\partial J}{\partial x_{i}} * \frac{x_{i, 0}}{J\left(x_{i, 0}\right)}
$$

Eq. 2

In Eq. 2, the terms ' $J$ ' and ' $x_{i}$ ' represent the model output and inputs respectively. The subscripts ' $i$ ' and ' 0 ' represent the input number and initial values respectively. During the calculation of the Local Sensitivity Index (LSI), considering only the partial derivatives resulted in a raw or unnormalized local sensitivity. In other words, the LSI calculated with only the partial derivatives was unit dependent. For example, the LSI was expressed as the percentage change in the FPEG efficiency per $\mathrm{mm}$ change in the cylinder bore. Thus, the partial derivatives were normalized with the respective initial parameters. After normalizing, the LSI values were relative and unitindependent. For example, the LSI was expressed as a percentage change in the FPEG efficiency per percentage change in the cylinder bore. In this investigation, the normalized LSI values were considered. In other words, the LSI values were unit-independent. All the 25 model inputs mentioned above were varied individually around the fundamental value mentioned in Table 2. The cost function was comprised of 10 model outputs. This cost function was used to understand the effect of the model inputs on different components of the FPEG system. The model outputs included indicated power, indicated efficiency, LEM electric power, FPEG system efficiency, effective stroke length, frequency, percentage of exhaust gas retained, heat transfer losses, exhaust heat losses, and conversion inefficiency losses. The indicated and the electrical characteristics provided the information related to the FPEG performance. The effective stroke length, frequency, and percentage of exhaust gas retained provided information about FPEG operating conditions. Finally, the heat transfer, exhaust heat, and conversion inefficiencies provided the details of major FPEG losses.

\subsubsection{Cylinder geometry \& flow conditions}

This section reported the LSI values obtained by varying one variable at a time. In this section, only cylinder geometry and flow conditions were considered. The parameters in this section were the cylinder bore, maximum stroke, scavenging pressure, exhaust pressure, and intake temperature. The initial or fundamental values of all these parameters were maintained the same 
as mentioned in Table 2. The range and the fundamental values for these parameters are mentioned in Table 3. In this case, only 15 samples were considered for each variable. Based on this, the model was evaluated 75 times for all the 5 parameters.

Table 3: Range \& fundamental values of cylinder geometry and flow inputs.

\begin{tabular}{|c|c|c|c|}
\hline Parameter & Range & Fundamental value & Units \\
\hline Cylinder bore & $30-45$ & 38 & $\mathrm{~mm}$ \\
\hline Maximum stroke length & $30-45$ & 30 & $\mathrm{~mm}$ \\
\hline Exhaust pressure & $0.95-1.01$ & 0.99 & $\mathrm{bar}$ \\
\hline Intake pressure & $1.01-1.20$ & 1.1 & $\mathrm{bar}$ \\
\hline Intake temperature & $300-350$ & 300 & $\mathrm{~K}$ \\
\hline
\end{tabular}

The results from the model evaluations were post-processed using a MATLAB® script. The normalized LSI values were calculated for all the 10 model outputs with respect to the 5 model inputs. The normalized LSI values are provided in Table 4 . The subscripts 'max' and ' $C$ ' represent the maximum value and conversion inefficiencies respectively. The effects of all these model inputs were provided with increasing input. For example, increasing the model input by $1 \%$ increased the model output by $1 \%$ or decreased the model output by $-1 \%$. Of all the five model inputs, the scavenging pressure had a positive effect on the FPEG performance. An increase in the scavenging pressure reduced the exhaust gas products retained in the combustion chamber. With the reduced exhaust gas retained, the controller increased the fuel input. The increase in the fuel input was to maintain the stoichiometric conditions in the cylinder. In other words, the amount of fuel and air mixture delivered was increased with increasing scavenging pressure. However, the exhaust pressure had a negative effect. This was again due to the increase in the exhaust gas retained. With increasing exhaust pressure, the exhaust pressure differential was reduced. The exhaust pressure differential referred to the difference in the cylinder and exhaust pressure. This exhaust pressure differential was responsible the blow down of the exhaust products. Due to the reduction in the exhaust pressure differential, the discharge of the exhaust products was reduced. Finally, to maintain the stoichiometric conditions, the controller reduced the fuel input. This fuel input further reduced the FPEG performance with increasing exhaust pressure.

Based on the normalized LSI values, a $1 \%$ increase in the scavenging pressure reduced the EGR by $9.1 \%$. The EGR from here onwards referred as the exhaust gas retained in the cylinder. It also 
increased the indicated and LEM power by $4.8 \%$ and $5.3 \%$ percentages respectively. This increase in the engine and LEM power output was mainly due to an increase in the fuel. However, the indicated and FPEG system efficiencies were improved by only $0.3 \%$ and $0.9 \%$. This was due to various losses in the system. Mainly, the fuel slip losses were increased with increased scavenging pressure. Moreover, the slightly high percentage improvement in LEM efficiency was due to the reduction in the conversion inefficiency. The conversion efficiency was reduced by $1.6 \%$ with an increase in the scavenging pressure. In contrast, with increasing the exhaust pressure, the exhaust pressure gradient was reduced. This reduced pressure gradient increased the amount of EGR by $5.7 \%$. The increased EGR value reduced the indicated and LEM power by $2.5 \%$ and $2.3 \%$.

Table 4: Normalized LSI values for model outputs based on cylinder geometry \& flow conditions.

\begin{tabular}{|c|c|c|c|c|c|c|c|c|c|c|}
\hline Parameter & $x$ & $f$ & $E G R$ & $P_{I C E}$ & $P_{L E M}$ & $\eta_{I C E}$ & $\eta_{F P E G}$ & $\eta_{H T}$ & $\eta_{E H R}$ & $\eta_{C}$ \\
\hline $\boldsymbol{P}_{\text {exhaust }}$ & -0.037 & -0.028 & 5.73 & -2.51 & -2.26 & -0.12 & -0.01 & 1.84 & -0.62 & -0.52 \\
\hline $\boldsymbol{P}_{\text {intake }}$ & 0.0001 & 0.01 & -9.09 & 4.79 & 5.33 & 0.25 & 0.9 & -3.7 & 1.52 & -1.57 \\
\hline Bore & 0.0995 & 0.066 & 1.27 & 0.20 & 2.11 & 0.15 & 0.4 & 2.70 & -0.47 & -0.69 \\
\hline $\boldsymbol{S}_{\text {max }}$ & 0.8923 & -0.025 & 0.1950 & 0.97 & 0.85 & 0.12 & 0.06 & -0.6 & 0.09 & 0.00 \\
\hline $\boldsymbol{T}_{\text {intake }}$ & -0.02 & $-0 . .22$ & 0.30 & -0.89 & -0.95 & -0.42 & -0.48 & 0.49 & -0.5 & 0.2 \\
\hline
\end{tabular}

When the intake temperatures were increased by $1 \%$, the peak cylinder temperatures were increased. As a result, the heat transfer losses were increased by $0.5 \%$. This increase in the heat transfer losses reduced the cylinder pressures and cylinder forces. These parameters, in turn, reduced the translator displacement in the upcoming cycles. With the reduced displacement, the EGR was increased due to low pressure gradient and low velocity at the EPO. Due to these reasons, the indicated and electrical powers were reduced by $0.9 \%$ and $1.0 \%$ per $1 \%$ increase in the intake temperature.

The main factors in calculating the LSI values were the goodness of the fit and the initial parameters. The initial values were obtained based on the fundamental case presented in Section 2.3. The variations in the normalized indicated power with respect to normalized intake pressure and temperatures are shown in Figure 75. From Figure 75, two sets of data were reported for pressure and temperatures. One set of data corresponded to the actual data obtained from the numerical model evaluations. The second set of data represented the curve fit used to calculate 
the LSI. Based on Figure 75, the scavenging pressure curve fit was more accurate than the intake temperature curve fit. The accuracy was defined by using coefficient of determination $\left(R^{2}\right)$ values. Though there were slight deviations, the $R^{2}$ values of these curve fits were found to be 0.99 and 0.97 . Similarly, all the LSI values considered the curve fits with $R^{2}$ values greater than 0.95 . The variation in the normalized indicated power output with respect to the normalized bore and maximum stroke is presented in Figure 76.

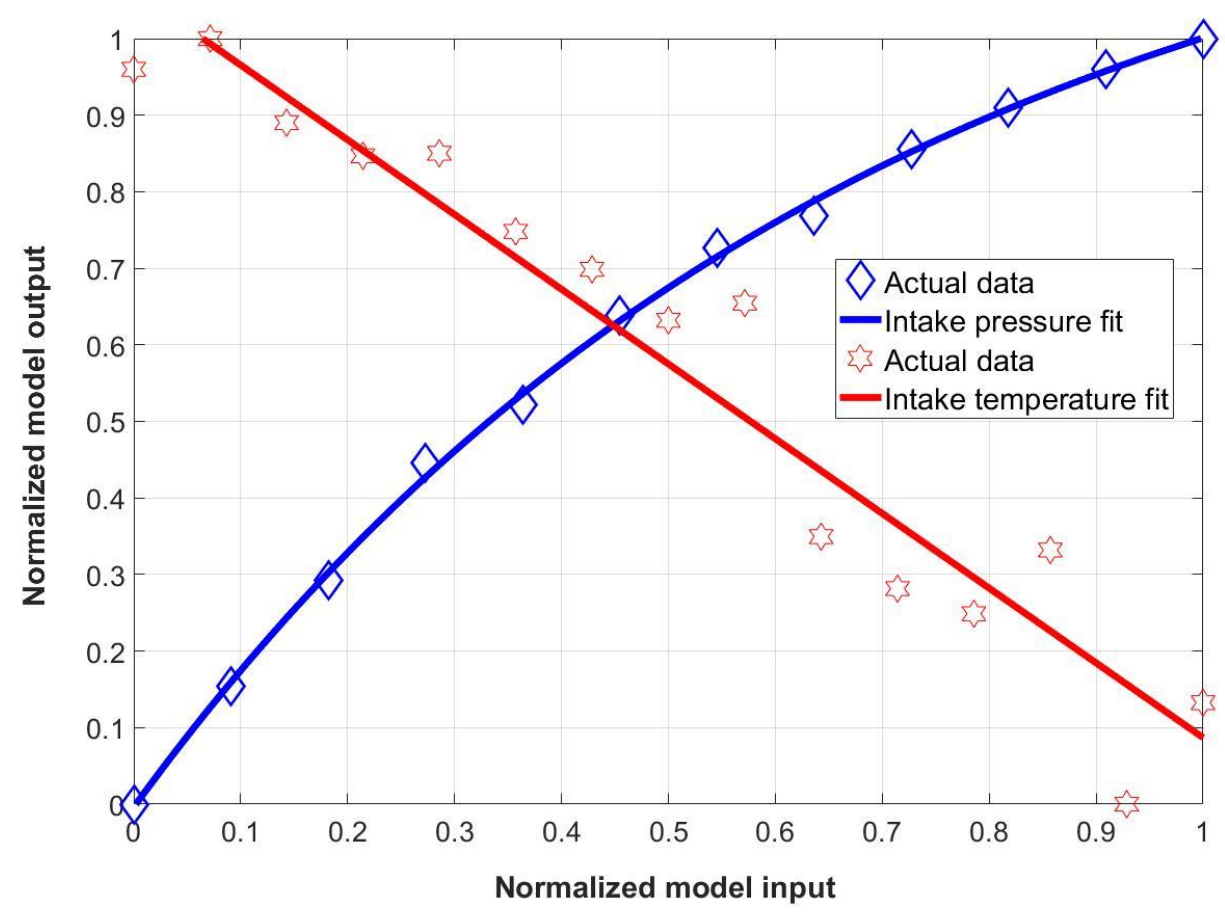

Figure 75: Variation in normalized indicated power with normalized scavenging and intake temperature.

From Figure 76, the normalized indicated power distribution followed a linear trend for cylinder bore. However, the maximum stroke followed a Fourier distribution of $2^{\text {nd }}$ order. This high order curve fitting was required especially for high $\mathrm{R}^{2}$ values. Moreover, the scatter in the maximum stroke was because of the variations in the outputs of multiple submodels. From Table 4, a $1 \%$ increase in the maximum stroke does not correspond to the $1 \%$ increase in the effective stroke. This was due to two factors. The first factor was the increase in frictional losses. The second factor was the increase in the minimum clearance value for a given compression ratio. Moreover, with the change in the maximum stroke, the port geometry was also changed. These changes further caused the variations in the cylinder forces. The effective displacement was calculated based on the resultant of these forces. Besides, the assumptions employed in various numerical 
submodels were also responsible for the slight reduction in the effective stroke length. In summary, the effective stroke was increased by $0.9 \%$ per $1 \%$ increase in the maximum stroke length. Finally, the cycle-to-cycle variations induced by various engine submodels caused the uneven shape in the output distribution in Figure 76.

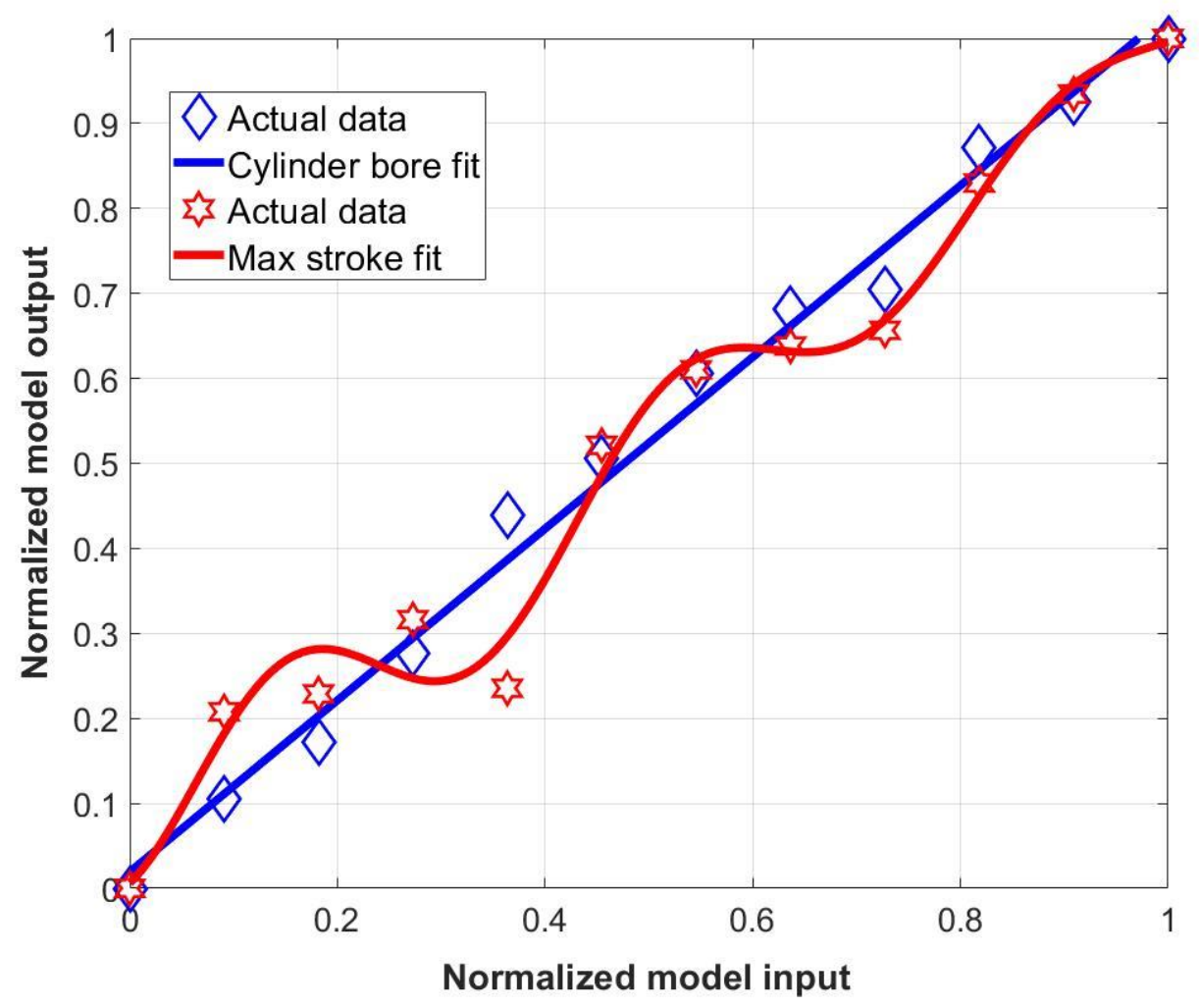

Figure 76: Variation in normalized indicated power with normalized bore and maximum stroke length.

\subsubsection{Exhaust \& scavenging port geometry}

In the numerical model, the exhaust and scavenging port dimensions were provided as inputs. These dimensions were defined in the numerical model as a percent of the maximum stroke length. For example, the exhaust port was located at 55\% of the maximum stroke from the zero location. In CSE, the port location, opening, and closing timings were defined by using crankshaft degrees. These opening and closing locations were fixed for a particular operating speed. However, in an FPEG system, the location of the initial position and effective stroke length defined the locations and timings. In other words, the port operations were directly linked to translator dynamics. By changing the initial position and dynamics, the port operations were either delayed or advanced. In this section, the exhaust port location, exhaust port width, exhaust port height, 
scavenging port location and scavenging port radius were varied one at a time. Similar to the previous case, the model was evaluated 15 times for each model input. In other words, a total of 75 model evaluations was considered for all the five model inputs. The range and the fundamental values for these parameters are mentioned in Table 5. The normalized LSI values obtained in this case are presented in Table 6.

Table 5: Range \& fundamental values of exhaust and scavenging port geometry.

\begin{tabular}{|c|c|c|c|}
\hline Parameter & Range & Fundamental value & Units \\
\hline Exhaust port location & $40-50$ & 42.5 & $\%$ \\
\hline Exhaust port width & $17.5-32.5$ & 25 & $\%$ \\
\hline Exhaust port height & $22.5-35$ & 27.5 & $\%$ \\
\hline Scavenging port location & $50-65$ & 57.5 & $\%$ \\
\hline Scavenging port radius & $12.5-25$ & 18.5 & $\%$ \\
\hline
\end{tabular}

Table 6: Normalized LSI values for various model outputs based on exhaust and scavenging port geometry.

\begin{tabular}{|c|c|c|c|c|c|c|c|c|c|c|}
\hline Paramete & $x$ & $f$ & $E G R$ & $P_{I C E}$ & $P_{L E M}$ & $\eta_{I C E}$ & $\eta_{F P E G}$ & $\eta_{H T}$ & $\eta_{E H R}$ & $\eta_{C}$ \\
\hline $\boldsymbol{E P}_{\boldsymbol{l}}$ & 0.000 & 0.001 & 0.003 & 0.117 & 0.014 & 0.03 & 0.007 & 0.006 & 0.003 & -0.005 \\
\hline $\boldsymbol{E} \boldsymbol{P}_{\boldsymbol{w}}$ & 0.000 & 0.000 & 0.000 & -0.00 & -0.00 & -0.00 & -0.00 & -0.001 & -0.00 & 0.000 \\
\hline $\boldsymbol{E} \boldsymbol{P}_{\boldsymbol{h}}$ & 0.000 & 0.000 & -0.01 & 0.003 & 0.018 & 0.0005 & 0.0007 & -0.002 & 0.000 & 0.000 \\
\hline $\boldsymbol{S} \boldsymbol{P}_{\boldsymbol{l}}$ & -0.02 & -0.39 & 0.90 & -2.87 & -2.25 & -0.007 & -0.008 & 0.003 & -1.75 & -0.58 \\
\hline $\boldsymbol{S} \boldsymbol{P}_{\boldsymbol{r}}$ & 0.000 & 0.01 & -0.78 & 0.305 & 0.45 & 0.3 & 0.32 & -0.31 & 0.11 & -0.07 \\
\hline
\end{tabular}

The subscripts ' $l$ ', ' $w$ ', ' $r$ ', and ' $h$ ' represent location, width, radius, and heights respectively. The exhaust port was considered as a projected rectangular opening. The scavenging port was modeled as both projected circular and rectangular openings in the numerical model. However, for the sensitivity analysis, it was considered as a projected circular opening on the cylinder liner. These port dimensions were mainly used in the gas exchange models. Especially in the gas exchange models, the port areas influenced the mass flow rates. Based on Table 6, the location and radius of the scavenging port had noticeable but minimal effect on the model outputs. The LSI values, in this case, were lower compared to the LSI values in Table 4. This was because of two reasons. The first reason was due to the narrow range considered for the model inputs as 
shown in Table 5. Changing the ports beyond these ranges resulted in an unstable FPEG operation. In other words, operating conditions should be changed to change the port geometry beyond these regions. The second reason was the minimal effect of these model inputs in defining the outputs.

Mainly, the delayed SPO reduced the amount of time available for the scavenging process. Moreover, with the reduced scavenging time, the amount of fuel and air mixture flow into the cylinder was also reduced. Due to the reduction in fuel and air mixture, the indicated and FPEG power outputs were reduced by $2.9 \%$ and $2.3 \%$. Similarly, the increase in the scavenging port radius increased the amount of intake mass flow rate. This was because of the increase in the intake flow area. Finally, the increased mass flow rate increased the indicated and LEM electrical power by $0.4 \%$. From Table 6 , the port geometry had a minimal scope for improving the FPEG performance.

\subsubsection{FPEG system design \& operating conditions}

In this section, the FPEG system design \& operating conditions like LEM efficiency, spring stiffness, and translator mass were considered. A total of 15 model inputs was considered in this case. Out of these 15 inputs, 4 of them were related to the HRR process. The HRR parameters included the Wiebe form factors, combustion efficiency, and duration. The next 5 of them were the Hohenberg heat transfer correlation coefficients. The final 6 of them were LEM efficiency, spring stiffness, moving mass, trapped compression ratio, equivalence ratio, and ignition timing. The range and the fundamental values of the operating conditions are mentioned in Table 7 . The same variable ranges were considered in the global sensitivity studies presented in Sections 4.4 and 4.5. The normalized LSI values obtained in this case are presented in Table 8.

In Table 8, the grey-colored cells represented the factors defining the HRR process. The orangecolored cells represented the heat transfer correlation coefficients. The green-colored cells represented the FPEG operating conditions. In the HRR process, the combustion efficiency was found to have a dominant effect. After combustion efficiency, the combustion duration, Wiebe form factor ' $a$ ', and Wiebe form factor ' $m$ ' followed the dominance order. The increase in the combustion efficiency had a positive effect on the FPEG performance. However, an increase in the combustion duration reduced the indicated and electrical power. 
Table 7: Range \& fundamental values of FPEG system operation conditions.

\begin{tabular}{|c|c|c|c|}
\hline Parameter & Range & Fundamental value & Units \\
\hline Wiebe form factor a & $4-6$ & 5 & - \\
\hline Wiebe form factor b & $1.5-3$ & 2 & - \\
\hline Combustion duration & $1.5-3$ & 2.25 & $\mathrm{~ms}$ \\
\hline Combustion efficiency & $80-95$ & 95 & $\%$ \\
\hline Hohenberg correlation a & $70-130$ & 97.5 & - \\
\hline Hohenberg correlation b & Neg $0.02-0.10$ & Neg 0.06 & - \\
\hline Hohenberg correlation c & $0.4-1.2$ & 0.8 & - \\
\hline Hohenberg correlation d & Neg $0.2-0.6$ & Neg 0.4 & - \\
\hline Hohenberg correlation e & $0.4-1.2$ & 0.8 & - \\
\hline LEM efficiency & $75-95$ & 75 & $\%$ \\
\hline Spring stiffness & $450-750$ & 558 & $\mathrm{kN} / \mathrm{m}$ \\
\hline Translator mass & $2-2.75$ & 2.75 & $\mathrm{~kg}$ \\
\hline Trapped compression ratio & $6.25-10.25$ & 8.25 & - \\
\hline Equivalence ratio & $0.9-1.1$ & 1.0 & - \\
\hline Ignition timing & $0-4$ & 4 & $\mathrm{~m} / \mathrm{s}$ \\
\hline
\end{tabular}

From Table 8, a 1\% increase in combustion efficiency improved the indicated and electrical power by $1 \%$. However, the indicated and system efficiencies were improved by $0.8 \%$. The $0.2 \%$ change between efficiency and power was due to the combined effects of friction, heat transfer, and exhaust heat losses. Increasing the combustion duration decreased the indicated and FPEG power output. The main reason for the reduction was due to the reduction in the cylinder pressure at TDC. This reduced pressure peak was because of the reduced MFB percentage at TDC. The variations in the MFB profiles with combustion duration were presented in our paper [2]. Moreover, with increasing the combustion duration, the exhaust heat rejection losses were also increased. This was due to the shift in the MFB profile later in the expansion stroke. Finally, the Wiebe form factors had a negligible effect on indicated and electrical power outputs. This was due to the constant combustion duration maintained for all the cases. Since the combustion duration was fixed, only the HRR distribution was changed. However, the combustion was finished within the same time in all cases. Because of this, the Wiebe form factors had a negligible effect on the FPEG performance. In other words, the modeling should change the combustion duration and efficiencies when Wiebe form factors were changed. These changes were considered in the global sensitivity studies for these model inputs in Section 4.4.3. 
Table 8: Normalized LSI values for various model outputs based on FPEG system operating conditions.

\begin{tabular}{|c|c|c|c|c|c|c|c|c|c|c|}
\hline Parameter & $x$ & $f$ & $E G R$ & $P_{I C E}$ & $P_{L E M}$ & $\eta_{I C E}$ & $\eta_{F P E G}$ & $\eta_{H T}$ & $\eta_{E H R}$ & $\eta_{C}$ \\
\hline$a$ & 0.002 & 0.006 & 0.03 & 0.07 & 0.07 & 0.03 & 0.04 & 0.11 & -0.05 & -0.02 \\
\hline$m$ & 0.000 & -0.01 & -0.01 & -0.02 & -0.00 & -0.01 & -0.00 & -0.13 & 0.03 & 0.01 \\
\hline$\eta_{\text {comb }}$ & 0.027 & 0.02 & -0.81 & 0.97 & 1.07 & 0.73 & 0.77 & 0.81 & 0.45 & -0.28 \\
\hline$t_{\text {comb }}$ & -0.006 & -0.02 & -0.04 & -0.16 & -0.19 & -0.12 & -0.13 & -0.36 & 0.16 & 0.03 \\
\hline$H_{a}$ & -0.004 & -0.002 & 0.11 & -0.15 & -0.16 & -0.14 & -0.15 & 0.66 & -0.13 & 0.036 \\
\hline$H_{b}$ & 0.002 & 0.000 & -0.08 & 0.12 & 0.12 & 0.07 & -0.57 & -0.57 & 0.06 & -0.02 \\
\hline$H_{C}$ & -0.009 & -0.003 & 0.25 & -0.35 & -0.4 & -0.34 & -0.38 & 1.87 & -0.27 & 0.11 \\
\hline$H_{d}$ & -0.015 & -0.017 & 0.55 & -0.7 & -0.87 & -0.62 & -0.70 & 4.18 & -0.32 & 0.51 \\
\hline$H_{e}$ & -0.006 & -0.003 & 0.20 & -0.3 & -0.34 & -0.24 & -0.26 & 1.21 & -0.20 & 0.06 \\
\hline$\eta_{L E M}$ & 0.000 & 0.000 & 0.000 & 0.00 & 1.00 & 0.00 & 1.00 & 0.00 & 0.00 & -2.88 \\
\hline$k$ & -0.048 & 0.359 & 0.92 & -0.23 & -0.25 & -0.07 & -0.07 & -0.03 & -0.04 & 0.08 \\
\hline$m$ & 0.011 & -0.59 & -0.74 & -0.30 & -0.17 & 0.35 & 0.35 & 0.53 & 0.16 & -0.08 \\
\hline$C R$ & 0.07 & 0.027 & -0.47 & 0.47 & 0.44 & 0.09 & 0.09 & -0.19 & 0.003 & 0.025 \\
\hline$\phi$ & 0.012 & 0.008 & 0.13 & 0.48 & 0.50 & -0.3 & -0.3 & -0.28 & -0.40 & -0.06 \\
\hline$I G N$ & -0.007 & -0.02 & -0.06 & -0.24 & -0.20 & -0.17 & -0.18 & -0.34 & 0.18 & -0.03 \\
\hline
\end{tabular}

In terms of heat transfer, of all the coefficients, the temperature coefficient $\left(H_{d}\right)$ was found to have a dominant effect. After $H_{d}$, the pressure coefficient $\left(H_{c}\right)$, velocity coefficient $\left(H_{e}\right)$, leading coefficient $\left(H_{a}\right)$, and volume coefficient $\left(H_{b}\right)$ followed the dominance order. From Table 8 , a $1 \%$ percentage change in ' $H_{d}$ ' reduced the indicated and electrical power by $0.7 \%$ and $0.9 \%$. The efficiencies were reduced by $0.6 \%$ and $0.7 \%$. In summary, these coefficients had a significant effect on the dynamics, performance, heat transfer losses in the subsequent cycles.

The final set of model inputs considered the main FPEG design \& operating parameters. The set included the LEM efficiency, spring stiffness, moving mass, trapped compression ratio, equivalence ratio, and ignition timing. In the numerical model, the LEM efficiency was provided as an input. The increase in LEM efficiency increased the LEM power output and FPEG efficiency. This was due to a reduction in the conversion losses. Moreover, the power and FPEG efficiencies were increased linearly with a linear increase in LEM efficiency. Unlike electrical power, the 
conversion inefficiency exhibited a non-linear effect. The reason for the non-linear effect was due to the presence of frictional losses along with the LEM losses in the conversion inefficiency. The conversion losses were reduced from $37.5 \%$ to $18.5 \%$ as the LEM efficiency was changed from $75 \%$ to $95 \%$. Further, from Table 8, the LEM efficiency only had an effect on the LEM power, efficiency, and conversion losses. No changes were observed in the engine operating conditions. Due to the same engine operating conditions, the power generated from the engine was not affected.

When spring stiffness was considered as model input, the deviations were mainly observed in the frequency and the EGR. Increasing the spring stiffness by $1 \%$, increased the frequency and EGR values by $0.4 \%$ and $0.9 \%$. For the same moving mass, doubling the spring stiffness should increase the frequency by $41.4 \%$. This value was in an ideal case without any combustion forces and losses. In the numerical model, the increase in the frequency was found to be $35.9 \%$. The reduction in combustion forces and increase in the losses reduced the frequency increase by $5.5 \%$. Moreover, the reduction in the combustion forces with increasing spring stiffness was mainly attributed to three reasons. The first reason was the increase in EGR with increasing stiffness. With increased EGR, the amount of fuel delivered was reduced. In other words, the controller reduced the fuel to maintain stoichiometric conditions. As a result, the indicated work and peak cylinder pressures were reduced with increasing spring stiffness.

Secondly, the ignition timing was retarded with increasing spring stiffness. This retarded ignition timing reduced the indicated power and increased EGR in the cylinder. Thirdly, the use of a constant combustion duration impacted the HRR profile. With increasing spring stiffness and constant combustion duration, the amount of fuel burned before TDC was reduced. Moreover, the $50 \%$ MFB location was retarded and occurred later in the expansion stroke with increasing stiffness. All these three factors reduced the indicated and electric power by $0.2 \%$ per $1 \%$ increase in the spring stiffness. With the reduction in the indicated power, the efficiencies were also reduced by $0.07 \%$.

For the same spring stiffness, doubling the moving mass should decrease the operating frequency by $70.7 \%$. This was again for the ideal case with no losses and combustion forces. In the numerical model, when the moving mass was doubled, the frequency was reduced by $59 \%$. In addition to the frequency, the indicated and electrical power outputs were reduced by $0.3 \%$ and $0.2 \%$ per $1 \%$ increase in the moving mass. The main reason for the power reduction was due to 
the reduction in the frequency. Even though the indicated power was reduced, the indicated work and peak cylinder pressure grew with increasing moving mass. One reason for the increase was due to the better blow down of the exhaust contents. The blow down was further increased the fuel delivered into the cylinder. This increased fuel also increased the amount of fuel burned. Moreover, the rate of change in the cylinder volume between two time steps was reduced with increasing moving mass. The reduced rate of cylinder volume change further increased the cylinder pressure rise. Moreover, the efficiencies per cycle were calculated on a fuel energy basis. As a result, the efficiencies were improved by $0.4 \%$ due to an increase in the indicated work.

For the remaining model inputs, increasing the compression ratio and equivalence ratio increased the engine and LEM power. In contrast, retarding the ignition timing closer to TDC reduced the indicated power. This was due to a delayed expansion process. With the reduction in the power output, the indicated and electrical efficiencies were also reduced with the retarded ignition timing. The variations in the normalized efficiency for different normalized inputs are presented in Figure 77. Increasing the compression ratio increased the indicated efficiency. Increasing the equivalence ratio from lean condition increased the indicated efficiency until the stoichiometric equivalence ratio. A further increase in the equivalence ratio over 1.0 reduced the indicated efficiency. Especially, in the rich operation, the efficiency was reduced because of increased fuel slip and combustion losses. For the ignition timing, the timing was too advanced initially. As a result, the indicated efficiency was less initially. With retarding the ignition timing to the MBT location, the indicated efficiency achieved its peak value. Further retarding the ignition timing shifted the location of peak pressure away from TDC. Consequently, the fuel energy potential was lost. This lost fuel energy potential reduced the indicated efficiency after the MBT timing.

In summary, the OAT method provided quantitative information about the effect of model inputs on the model outputs. Moreover, only one input was varied at the time. In terms of flow conditions, the intake pressure was found to have a dominant effect on electrical power. This was due to the increased fuel flow rate into the cylinder. In terms of bore and stroke, the cylinder bore had a dominant effect on power output. This was due to the increased frequency and cylinder forces. The effect of the port locations and geometry had a negligible effect on the output power. In terms of FPEG operating conditions, the combustion efficiency had a dominant effect on output power followed by equivalence ratio, compression ratio, spring stiffness, and moving mass. These conclusions were only true when the interactions between the model inputs were not considered. However, these OAT studies were helpful in understanding the system variations. Since the actual 
FPEG systems have input interactions, global sensitivity studies were required. Initially, the global sensitivity studies were performed on all these 25 parameters. These studies were aimed at understanding the interactions between the model inputs. Based on the initial global sensitivity studies, seven model inputs were considered for a final global sensitivity study. The procedure for selecting these seven model inputs was explained in the upcoming section.

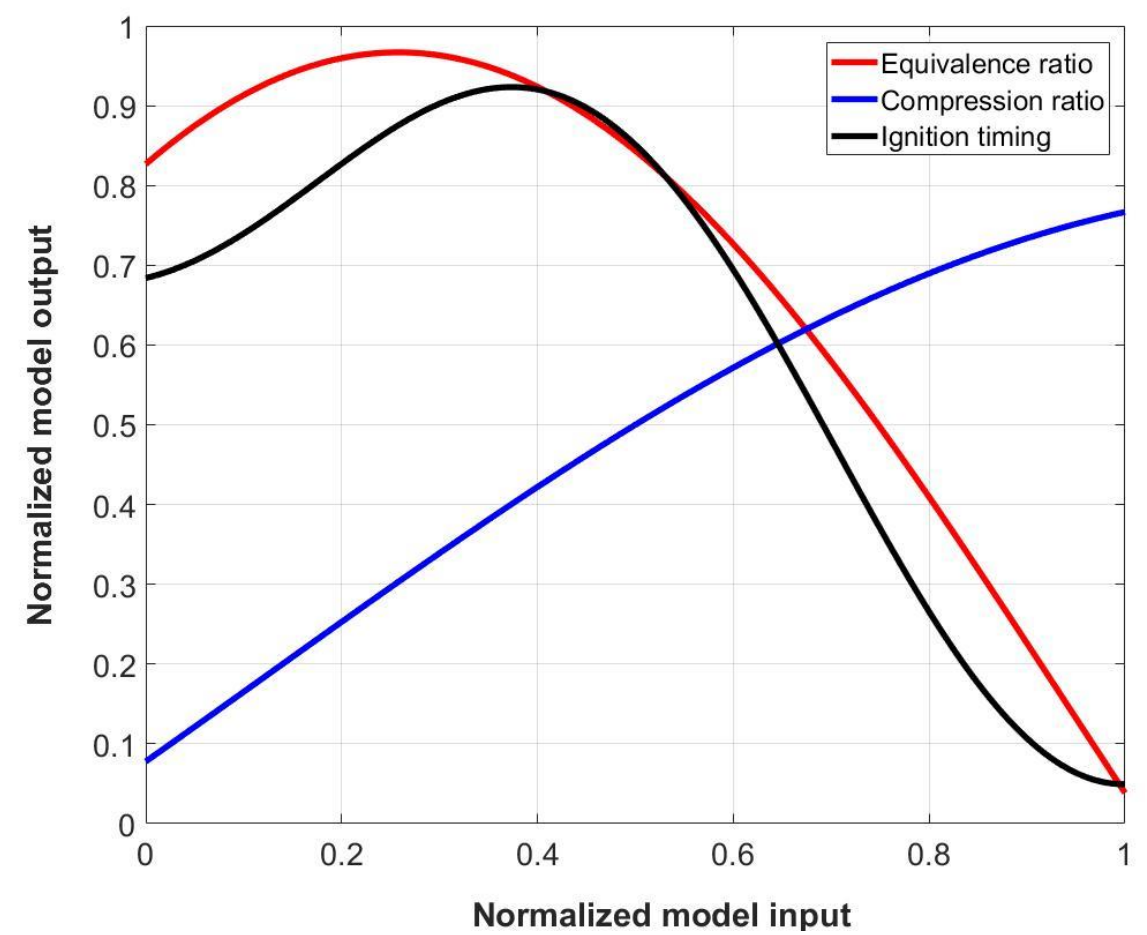

Figure 77: Variation in normalized indicated efficiency with normalized equivalence ratio, compression ratio and ignition timing.

\subsection{Global sensitivity studies}

In the local sensitivity studies, the relationship between the model inputs and model outputs was understood. However, the combined effects of the model inputs were poorly understood. Moreover, the LSI values were true only if the input and output fit was accurate. To overcome these disadvantages, a global sensitivity study with the Spearman correlation method was considered [120, 122]. The Spearman correlation method used the ranked transformation methods to calculate the sensitivity indexes [120]. Initially, this method arranged the data in the order of their ranks. The smallest model output was assigned a rank value of 1 . Later, the ranks 
were assigned for all the model outputs in the increasing order. Finally, the largest model output was assigned a rank value of $\mathrm{N}$. If the multiple model outputs had the same value, an average rank was assigned for those model outputs. After organizing the data, the correlation methods were used to define the coefficients. Unlike the standard methods, the correlations were applied to the ranks rather than the actual model outputs.

In the global sensitivity study, the Spearman coefficients were calculated for all the inputs considered in the OAT studies. However, unlike the local sensitivity, a total of 7500 global model evaluations were considered in this case. These 7500 samples were obtained by using a uniform probability distribution with the Sobol sampling method [121]. Unlike the OAT method, multiple model inputs were varied at a time in the global sensitivity methods. Specifically, the partial Spearman correlation coefficients were considered in this investigation [122]. Partial coefficients were considered when more than one model input was changed at a time. This partial Spearman correlation coefficients calculated the Global Sensitivity Index (GSI). Moreover, the effects of all other model inputs were excluded in calculating the GSI. In other words, the partial correlation between the model input $\left(x_{1}\right)$ and the model output $(y)$ for a given set of model inputs $\left(x_{1}, x_{2}, x_{3}, x_{4}, x_{5}\right)$, is the connection between the errors of $x_{1}$ and $y$. These errors were obtained from the regression of $x_{1}$ and $y$ with all other model inputs. The corresponding mathematical equations for calculating the Spearman coefficient are provided in Eq. 3 [120].

$$
\begin{gathered}
\rho_{x_{i} y \mid z}=\frac{\rho_{x_{i} y}-\rho_{x_{i} z} * \rho_{y z}}{\sqrt{\left(\left(1-\rho_{x_{i} z}{ }^{2}\right)\left(1-\rho_{y z}{ }^{2}\right)\right)}} \\
\rho_{x y}=\frac{\sum_{j=1}^{N}\left(x_{j}-\bar{x}\right)\left(y_{j}-\bar{y}\right)}{\sqrt{\left[\left(\sum_{j=1}^{N}\left(x_{j}-\bar{x}\right)\right)\left(\sum_{j=1}^{N}\left(y_{j}-\bar{y}\right)\right)\right]}} \\
\rho_{x y}=\frac{\operatorname{cov}(x, y)}{\sigma_{x} \sigma_{y}} \\
z \in \bigcup_{j=1, j \neq i}^{k} x_{j}
\end{gathered}
$$

Eq. 3

In Eq. 3, the term ' $z$ ' is the complementary set of model inputs other than $x_{i}$. The term ' $\rho_{x y}$ ' represent the strength of the relationship between a model input and model output. This strength 
was obtained by calculating the covariance and individual standard deviations $(\sigma)$. The terms $\bar{x}$ and $\bar{y}$ represent the mean of selected input and outputs respectively. The term 'j' represents the number of inputs selected for the global sensitivity study.

\subsubsection{Cylinder geometry \& flow conditions}

In this section, the results were obtained by varying all the cylinder geometry and flow conditions at a time. The set of parameters included the cylinder bore, stroke length, scavenging pressure, exhaust pressure, and intake temperature. Based on the sequences generated by the Sobol algorithm, the numerical model was evaluated. In this case, only two model output variations were presented. The selected model outputs were the indicated power and FPEG system efficiency. However, the variations in the other model outputs were also analyzed while making the conclusions. The variation in the LEM power output with respect to the change in cylinder bore is shown in Figure 78.

Unlike the linear distribution in Figure 76, the variations in the LEM electrical power were nonlinear. The cases with indicated power closer to $0 \mathrm{~W}$ represented the cases of failed evaluations. Moreover, Figure 76 also contained a lot of interactions. The interactions were mainly due to the change in multiple submodel outputs within the numerical model. For example, the change in the cylinder bore changed the cylinder forces for the same compression ratio. This change in the cylinder forces further changed the dynamics. Later, these dynamics changed the gas exchange, friction, heat transfer, and control submodels. As a result, an interdependency of variables was observed. Moreover, the distribution in Figure 78 only provided the information about the extent of interactions. However, it failed to provide the information of the dominant model input in causing those interactions. The statistical equation-based partial Spearman correlation coefficients provided this information. The Spearman coefficients for these model inputs with respect to indicated power are provided in Figure 79. The distribution in Figure 79 is called a tornado plot.

In general, the Spearman coefficient values were found to be in-between -1 and +1 . The positive value indicates the increase in the model output. The negative value implies the decrease in the model output. The closer the Spearman coefficient value to 1 , the higher the dominance of the model input on the model output. From Figure 79, of all the model inputs, the scavenging pressure was found to be dominant. Increasing the scavenging pressure supercharged the system. The 
supercharging increased the amount of fuel and air trapped inside the combustion chamber. As a result, this supercharging improved the indicated power output.

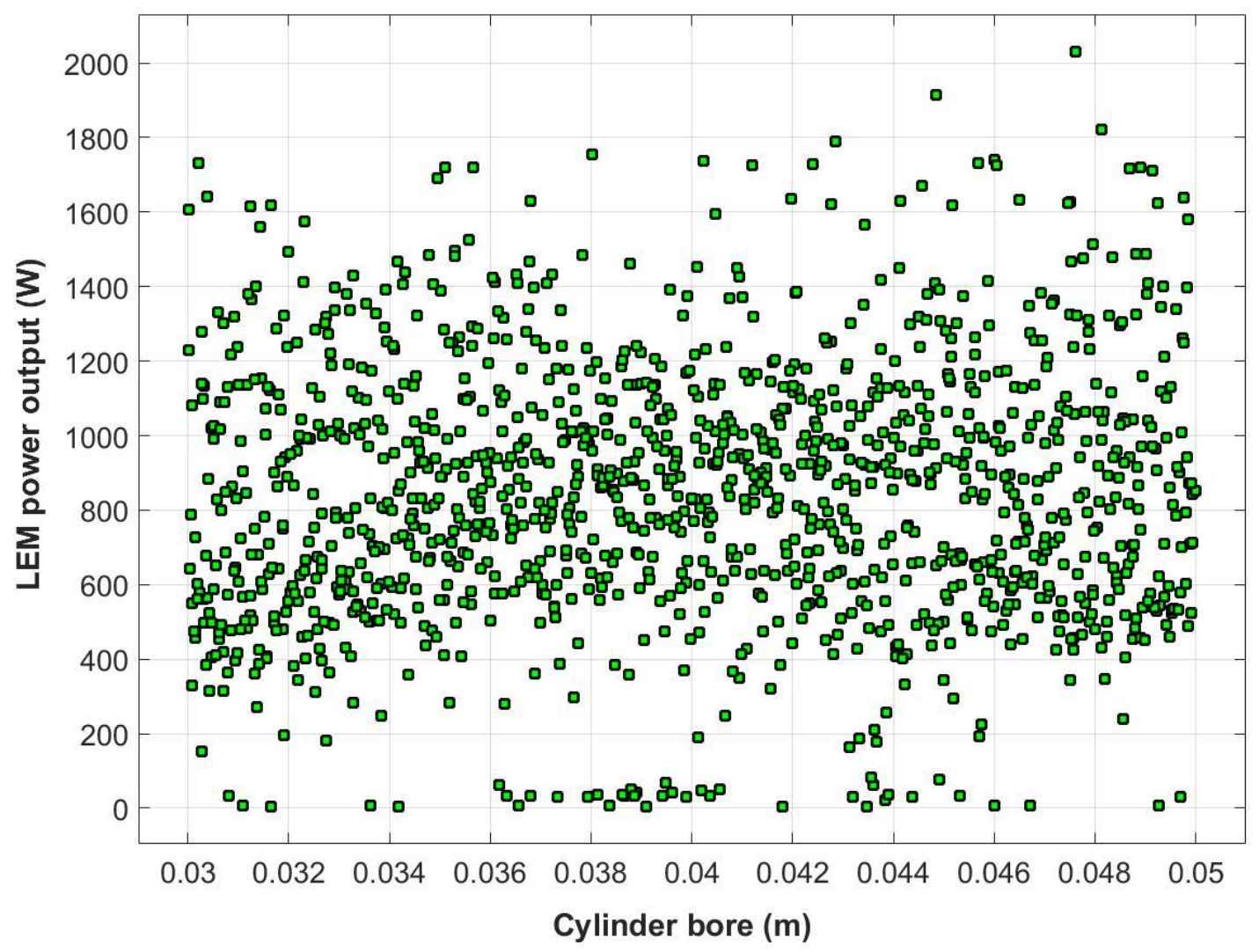

Figure 78: Variation in the LEM power output with cylinder bore in global sensitivity analysis.

After intake pressure, the effective stroke and cylinder bore were found to have high Spearman coefficients for the indicated power. Increasing the effective stroke for the same frequency increased the indicated work. The combustion forces and indicated work increased with an increase in the cylinder bore. This increase in the indicated work further increased the indicated power for the same operating frequency. On the negative side, the exhaust pressure was found to have a dominant effect on the indicated power. With an increase in the exhaust pressure, the pressure gradient between the cylinder and exhaust pressure was reduced. This decrease in the pressure gradient increased the amount of burned products inside the combustion chamber. With an increase in the EGR, the amount of fuel delivered was reduced. The fuel was reduced to maintain the stoichiometric conditions. This reduction in the amount of fuel delivered reduced the 
indicated power with increasing exhaust pressure. Finally, of all these parameters, the intake temperature was found to have the least dominant effect on the indicated power. The Spearman coefficients for these model inputs with respect to FPEG system efficiency are presented in Figure 80 .

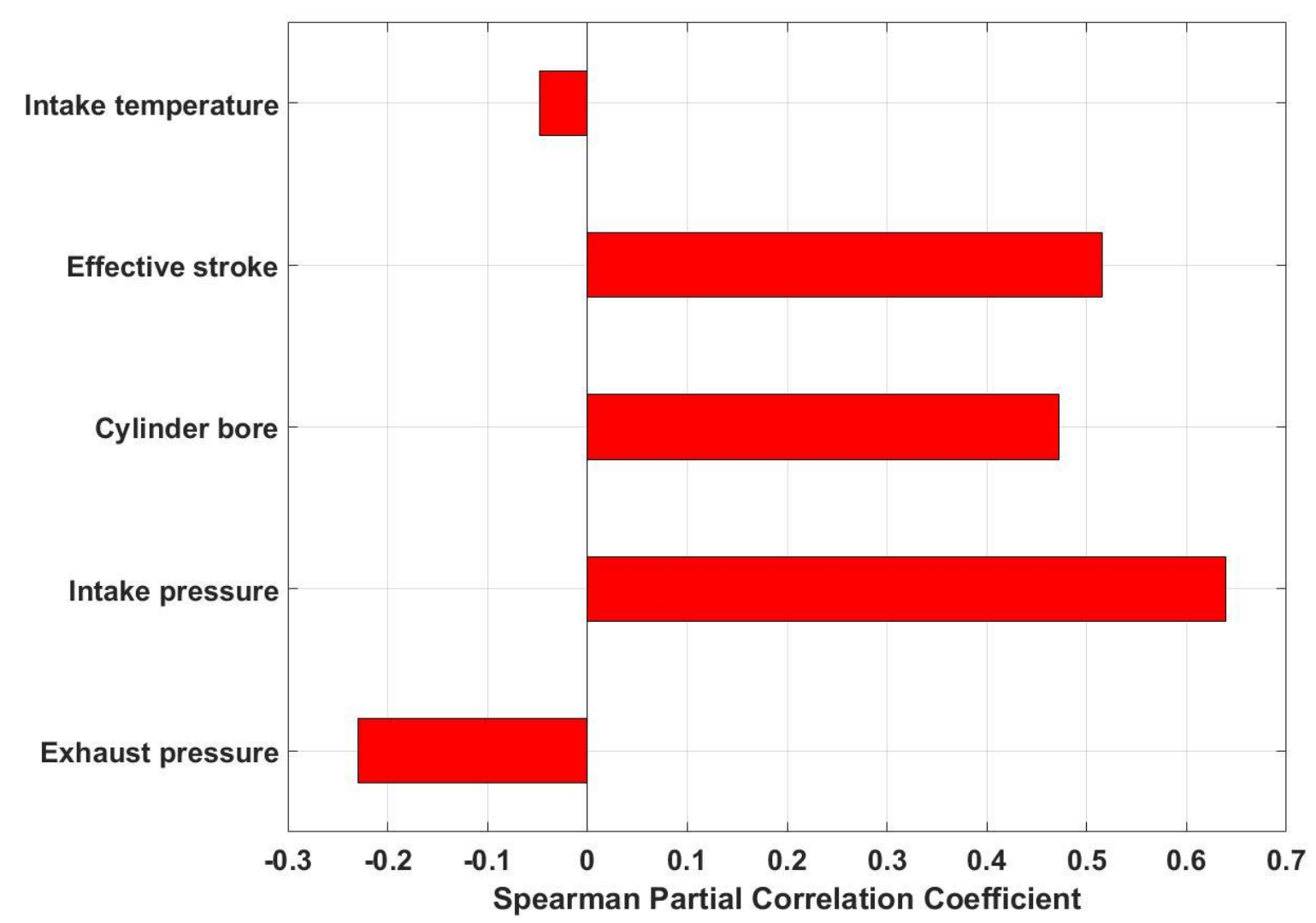

Figure 79: Spearman correlations for indicated power output with cylinder geometry and flow conditions.

Though the scavenging pressure increased the indicated power, it had a negative effect on the FPEG efficiency. This was due to an increase in the exhaust heat rejection and fuel slip losses with increasing the scavenging pressure. Moreover, the frictional losses were also increased with an increase in the cylinder pressures. All the three factors together reduced the FPEG system efficiency with the scavenging pressure. With an increase in the bore and stroke, the FPEG efficiency was also increased along with the indicated power. The effective stroke was found to have a dominant effect compared to the cylinder bore. The relative reduction in the FPEG efficiency due to the cylinder bore was due to increased frictional and heat transfer losses. The 
intake temperature had the highest negative dominant effect on the FPEG efficiency. The primary reason for the negative dominance was due to an increase in the combustion temperatures. These cylinder temperatures further increased the heat transfer losses in the system. Finally, the exhaust pressure was found to have the least dominant effect on the FPEG system efficiency. This was due to the reduction in the exhaust heat losses as more EGR was retained in the system.

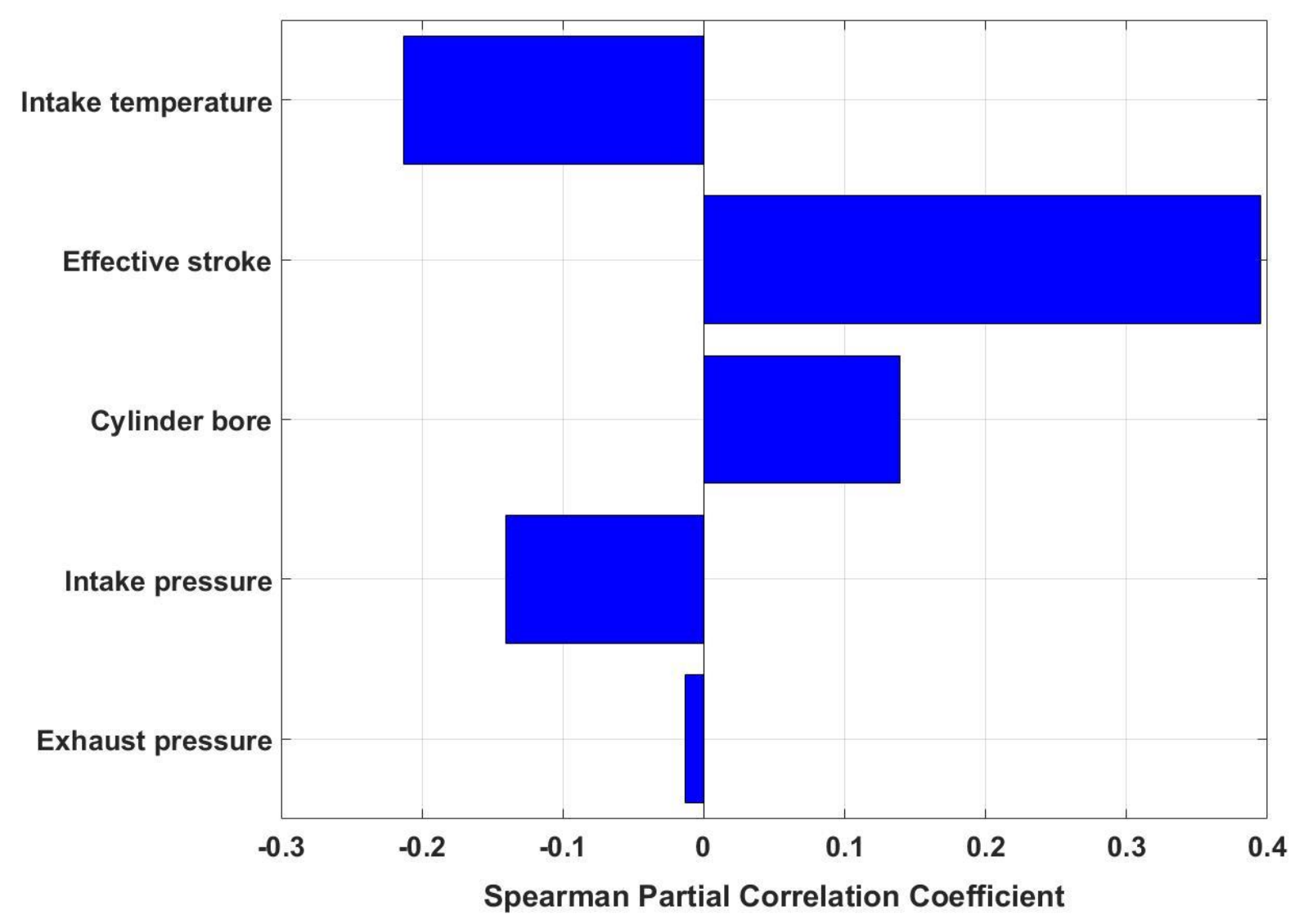

Figure 80: Spearman correlations for FPEG system efficiency with cylinder geometry and flow conditions.

\subsubsection{Exhaust \& scavenging port locations}

In this section, the global sensitivity analysis was carried out for the exhaust and scavenging port geometries. The Spearman coefficients for the port geometry with respect to indicated power and FPEG system efficiencies are shown in Figure 81. 


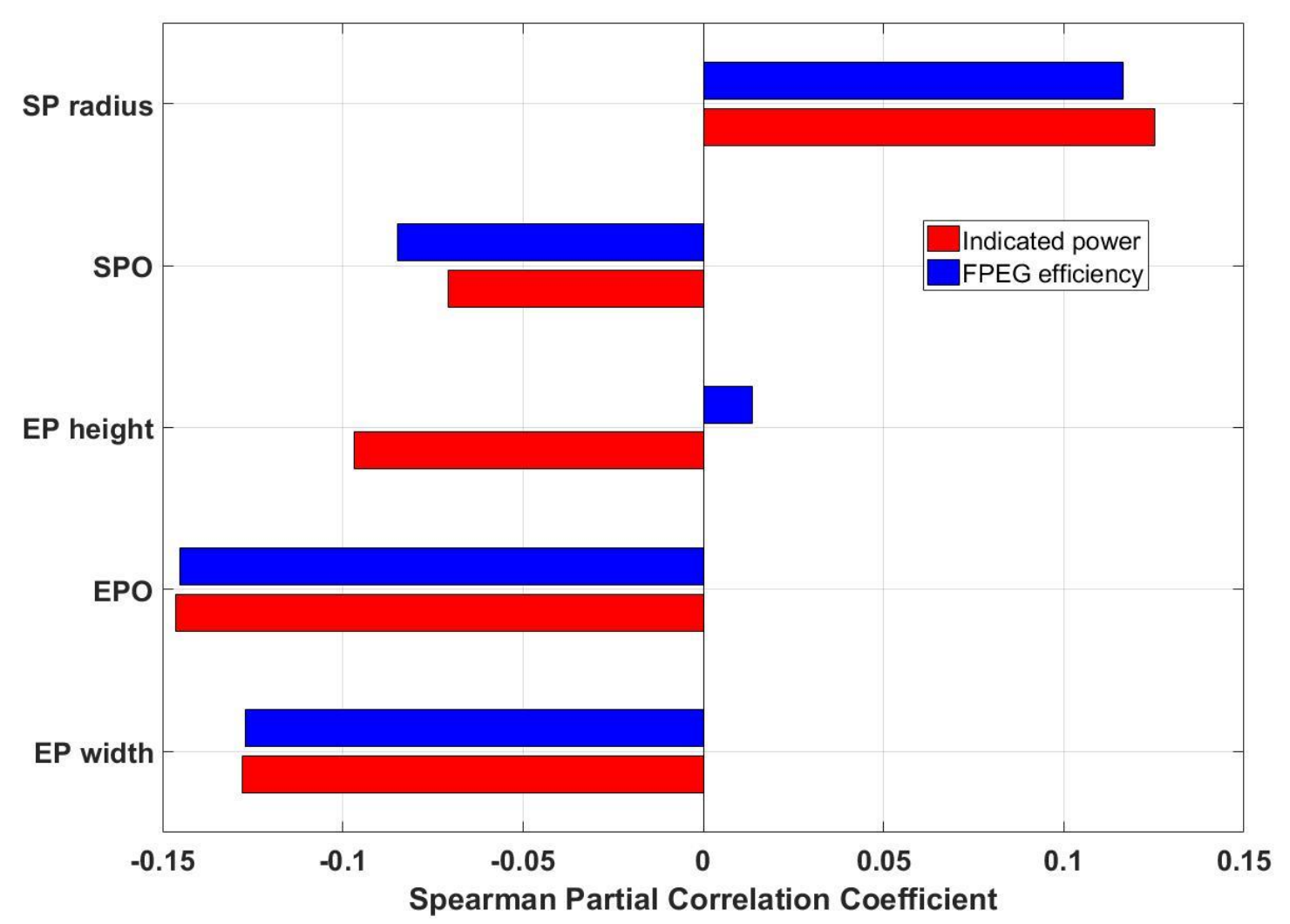

Figure 81: Spearman correlations for indicated power and FPEG system efficiency with varying port location and geometry.

Unlike the previous case, the Spearman coefficients were as low as 0.15 . These values represent the minimal effect of port geometry on the FPEG performance. Of all the model inputs, the scavenging port radius was found to have a dominant effect on the indicated power and FPEG efficiency. This was due to more air and fuel mixture trapped inside the combustion chamber. This increase in the fuel and air mixture also increased the HRR. This HRR further improved the indicated power. On the negative side, the EPO location was found to have a dominant effect. Firstly, the delayed EPO utilized the expansion potential of the combustion gases. However, the amount of EGR inside the cylinder was also increased with the delayed EPO. This increase in the EGR reduced the fuel input and mass of fuel burned in the successive cycles. On the whole, delaying the EPO and SPO reduced the indicated power. However, the reduction in the indicated power was relatively less when compared to the cylinder geometry and flow conditions. 


\subsubsection{FPEG system design \& operating conditions}

In this section, the FPEG operating conditions were varied globally. Similar to the OAT method, the HRR, heat transfer, LEM, and spring conditions were varied. Moreover, in the OAT method, the spring stiffness and moving mass were considered as independent parameters. However, in this case, they were considered as dependent parameters. In other words, the change in the spring stiffness also represented the change in the moving mass. To attain this, the translator mass was divided into two components. The two components were the static and dynamic masses. The static mass represented the mass of permanent magnets and piston assembly. The dynamic mass represented the moving mass related to the spring stiffness. In this investigation, the dynamic mass was considered as $20 \%$ of the individual spring mass. For example, if 20 springs were considered in the experimental FPEG system with an individual spring mass of 300 $\mathrm{gm}$. The total dynamic mass for that system was found to be $1.2 \mathrm{~kg}$. This dynamic mass was changed with spring stiffness. The Spearman correlation coefficients for HRR parameters with

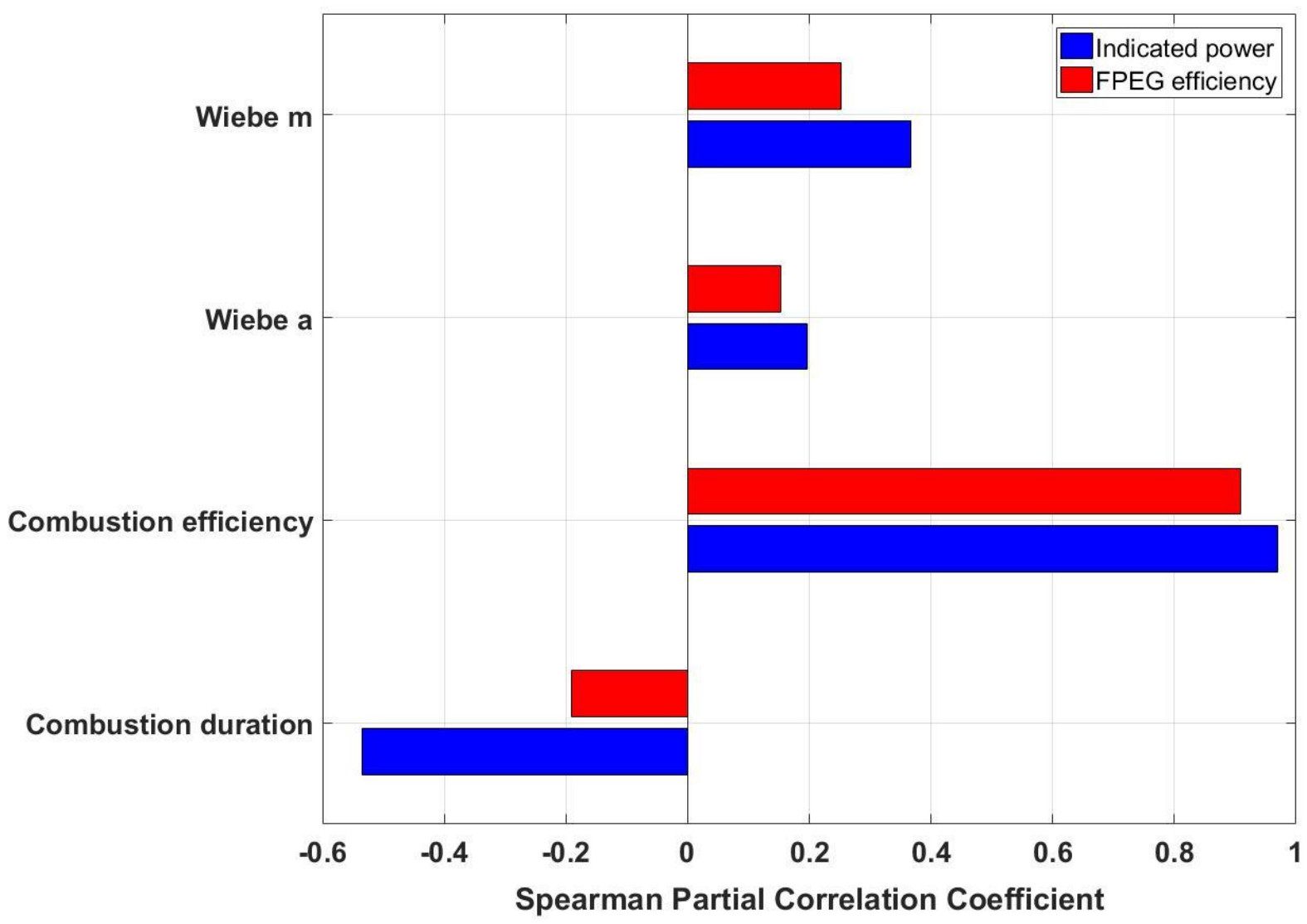

Figure 82: Spearman correlations for indicated power and FPEG system efficiency with combustion heat release parameters. 
Next to the combustion efficiency, the combustion duration was found to have a dominant effect on the indicated power and FPEG efficiencies. An increase in the combustion duration reduced the indicated power and FPEG efficiencies. With an increase in the combustion duration from 1.5 $\mathrm{ms}$ to $3 \mathrm{~ms}$, the indicated power was reduced from $1.6 \mathrm{~kW}$ to $1.4 \mathrm{~kW}$. This was due to two reasons. Firstly, for a short combustion duration, most of the fuel was burned before TDC. As a result, the trapped compression ratio and frequencies were increased for a short combustion duration. Secondly, with increased combustion duration, the combustion process was delayed. Consequently, the fuel energy potential was lost. In other words, the percentage of fuel burned before TDC was reduced. Moreover, the exhaust heat rejection losses were also increased with the delayed combustion process. Due to these exhaust heat rejection losses, the FPEG system efficiency was reduced more than the indicated power.

When Wiebe form factors were considered, the form factor ' $m$ ' was found to have a dominant effect. This form factor ' $m$ ' defines the shape of HRR. For a low value of form factor ' $m$ ', the $50 \%$ MFB location was found to occur before TDC. The presence of the 50\% MFB location before TDC resulted in the negative work on the piston. As the form factor ' $m$ ' was increased, the 50\% MFB occurred after TDC. This shift improved the indicated work and power outputs. The Wiebe form factor 'a' corresponds to the maximum percentage of fuel burned. At a given time equal to combustion duration, the maximum MFB described by the Wiebe function was less than unity by a factor of $e^{-a}$. For instance, at the time equal to combustion duration, the maximum MFB of $99.3 \%$ was obtained for ' $a$ ' value of 5 . Similarly, for 'a' value of 4 , the maximum MFB was found to be $98.2 \%$. The higher the value of form factor ' $a$ ' the closer the maximum MFB to unity. From Figure 82, increasing the form factor ' $a$ ' increased the indicated power and FPEG system efficiency. This increase was due to an increase in the maximum MFB closer to unity. This increase in the fuel burned further increased the cylinder pressures and translator velocities. On a final note, excluding the combustion efficiency, the combustion duration was found to have the dominant effect when only the HRR parameters were considered.

Next, the global sensitivity studies were performed on the Hohenberg heat transfer coefficients. The Spearman coefficients for Hohenberg heat transfer coefficients with respect to indicated power and FPEG efficiencies are shown in Figure 83. Similar to the OAT method, the increase in the heat transfer coefficients reduced the indicated power and FPEG system efficiencies. This was due to an increase in heat transfer losses. Of all the heat transfer coefficients, the temperature coefficient had the dominant effect. Increase the temperature coefficient increased 
the heat transfer coefficient which further increased the heat transfer losses. The temperature coefficient dominance was followed by pressure, volume, velocity, and leading coefficients.

Finally, the global sensitivity analysis was conducted on the FPEG operating conditions. The parameters include the LEM, spring, and engine operating conditions like equivalence ratio, compression ratio, and ignition timing. Moreover, the stroke length was also considered in this case. Unlike the previous case, the spring stiffness and moving masses were considered as dependent inputs. In other words, the change in the spring stiffness accompanied the change in the dynamic moving mass. While an increase in the spring stiffness was beneficial, an increase in the moving mass was detrimental to the frequency. The Spearman coefficients for FPEG operating conditions with respect to indicated power and FPEG efficiencies are shown in Figure 84.

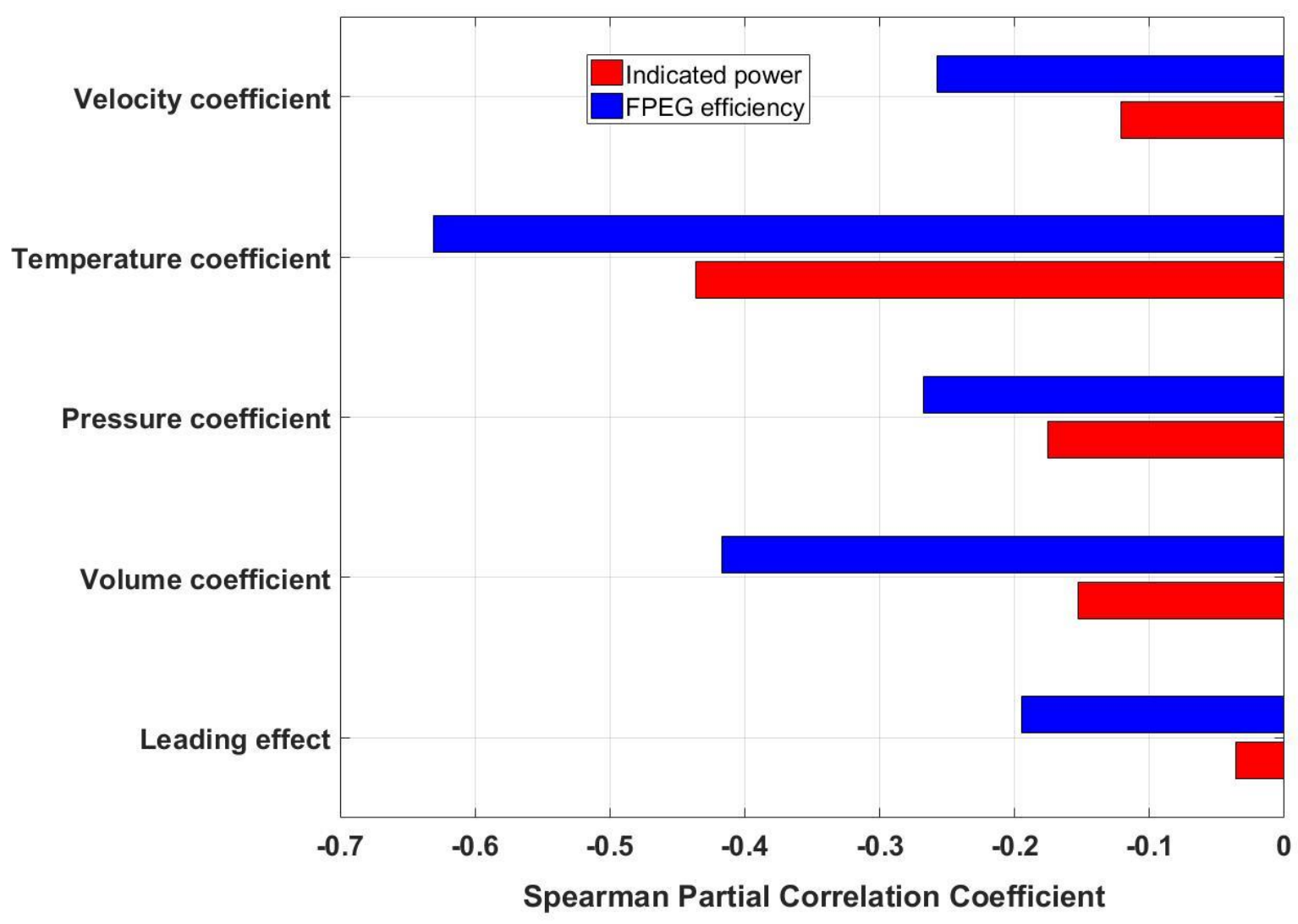

Figure 83: Spearman correlations for indicated power and FPEG system efficiency with heat transfer correlation coefficients. 
Of all the operating conditions, the stroke length and spring stiffness were found to have a dominant effect on the indicated power and FPEG efficiency. Increasing the spring stiffness increased the frequency. This frequency increase further increased the engine power output. With an increase in the stroke length, the translator velocities were increased. Moreover, the translator covered more cylinder volume with increasing stroke length. As a result, the indicated work and power outputs were increased with increasing stroke length. An increase in the indicated power also increased the LEM electrical power. On the negative side, an increase in the translator velocities increased the frictional losses in the system. These frictional losses impacted the conversion of indicated power to electrical power. Owing to these increased conversion losses, a lower Spearman coefficient was obtained for FPEG efficiency when compared to the indicated power.

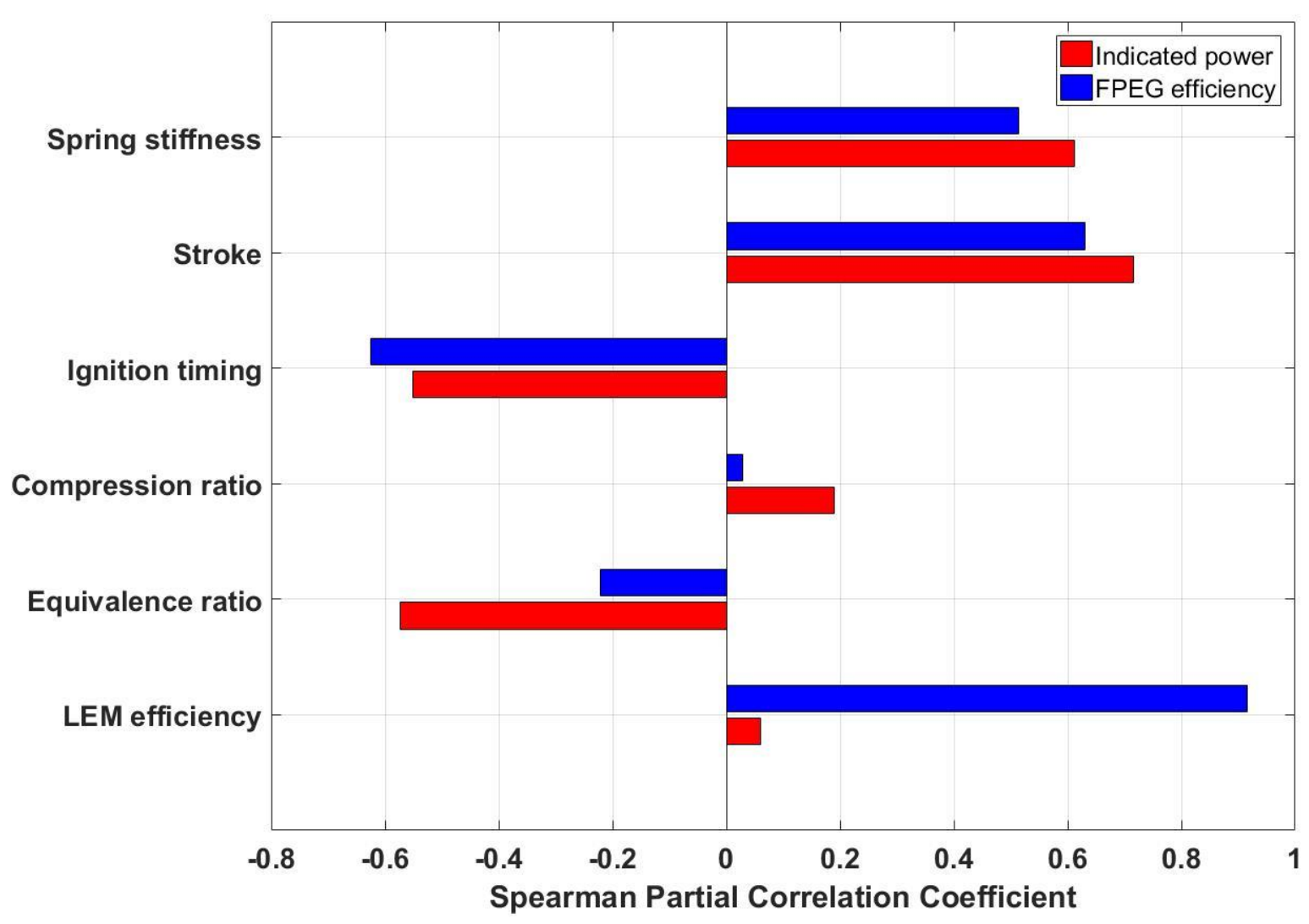

Figure 84: Spearman correlations for indicated power and FPEG system efficiency FPEG operating conditions. 
On the whole, when the model inputs shown in Figure 84 were considered, the stroke length was found to have the dominant effect. Retarding the ignition timing reduced the indicated power and FPEG efficiencies. This was due to a loss in fuel energy potential. Similarly, increasing the equivalence ratio increased the FPEG performance until the stoichiometric conditions. Later, a further increase in the equivalence ratio reduced the indicated power output and FPEG efficiencies. An increase in the trapped compression ratio increased the indicated power and FPEG efficiencies. However, the increase in efficiency was far less than the increase in the indicated power. This was mainly because of the increase in heat transfer and frictional losses. These losses reduced the corresponding increase in the FPEG efficiency with the compression ratio increase. Finally, an increase in the LEM conversion efficiency increased the FPEG system efficiency almost linearly. Moreover, the LEM conversion efficiency acted on the conversion from indicated to electric energy. As a result, minimal improvement in the indicated power was observed.

Finally, the results obtained in the section were used to rank the model inputs in the order of their dominance. This was done by screening the model inputs in each section. Of the 25 model inputs, seven of them were considered for the final global sensitivity study. The segregation process and the results from the final sensitivity study are reported in the upcoming section.

\subsection{Final global sensitivity studies}

This section reported the results from the final sensitivity studies of seven model inputs. The basis for the final sensitivity studies was obtained from screening the results presented in Sections 4.3 and 4.4. The main objective of the final sensitivity study was to utilize the numerical model for improving the experimental FPEG performance. Hence, the model inputs within the scope of the experimental prototype were considered for the final global sensitivity analysis. For example, the Hohenberg heat transfer coefficients were dependent on pressure, temperature, and dynamics. Hence, the Hohenberg coefficients were not considered for the final global sensitivity study. Similarly, the Wiebe form factors were not considered. This is because these factors were dependent upon the ignition timing and charge conditions. The port locations can be changed experimentally by using head spacing. However, the port geometry had minimal effect on the FPEG performance. Therefore, the port geometry was eliminated for the final global sensitivity study. In other words, the port geometry was fixed for the final sensitivity study. However, the port operations were directly linked to translator dynamics. 
During, the OAT method, both spring stiffness and mass were considered as independent variables. However, for the final global study, they were considered dependent. Therefore, only the spring stiffness was changed. The moving mass was varied linearly with the spring stiffness in the numerical model. In terms of ignition timing, an MBT timing algorithm was developed. This algorithm evaluated the numerical model at the optimized timing for all the cases. More details regarding the MBT timing algorithm are presented in Chapter 5. Finally, four model inputs, i.e. intake temperature, LEM efficiency, combustion efficiency, and equivalence ratio were maintained fixed. This was due to the liner effect of combustion and LEM efficiencies on FPEG performance. Hence, these parameters were excluded from the final global study. Moreover, the equivalence ratio created substantial power losses and impacted the spring forces. As a result, the stoichiometric condition was considered for the final global sensitivity study.

After excluding and fixing a few model inputs, seven model inputs were found to be remaining. The remaining model inputs were the scavenging pressure, exhaust pressure, cylinder bore, spring stiffness, stroke length, trapped compression ratio, and combustion duration. The scavenging and exhaust pressure variations were considered with the objective of using resonant exhaust and intake systems [123]. A study by Darzi showed the improvement in brake power with the optimized resonant intake and exhaust systems [117]. In a similar way, the spring design enabled the systems with different spring stiffness. The systems can be ranged from weak to spring dominant cases. In other words, the spring design allows the engine to be operated on a wide frequency range. The stroke length, cylinder bore, and the trapped compression ratio were considered to incorporate variable FPEG conditions. Considering these reasons, the abovementioned seven model inputs were considered for the final global sensitivity analysis study.

With these seven inputs, the numerical model was evaluated for 1500 samples. These samples were generated by the Sobol space filling algorithm. Later, the Spearman coefficients were used for ranking the inputs in the order of their dominance. In general, two different cases were considered in the final global sensitivity study. In the first case, the stroke length was considered to be variable for a given compression ratio. This was done by using a trapped compression ratio controller devised in Section 5.4.3. By using this controller, the system was made to run at the constant compression ratio. However, the BDC location was not controlled. In the second case, the stroke length was fixed. The effective stroke length of $30 \mathrm{~mm}$ was considered. The fixed stroke was maintained by using a dead center clearance setpoint controller devised in Section 5.5.4. 
Using this controller, the FPEG system was made to run within specified dead centers similar to the CSE.

\subsubsection{Variable stroke length}

In the section, the cylinder bore, effective stroke, scavenging pressure, exhaust pressure, spring stiffness, combustion duration, and compression ratio were considered. The range and the fundamental values for these parameters are shown in Table 9. The Spearman correlation coefficients of these seven model inputs with respect to indicated power and FPEG efficiencies are provided in Figure 85.

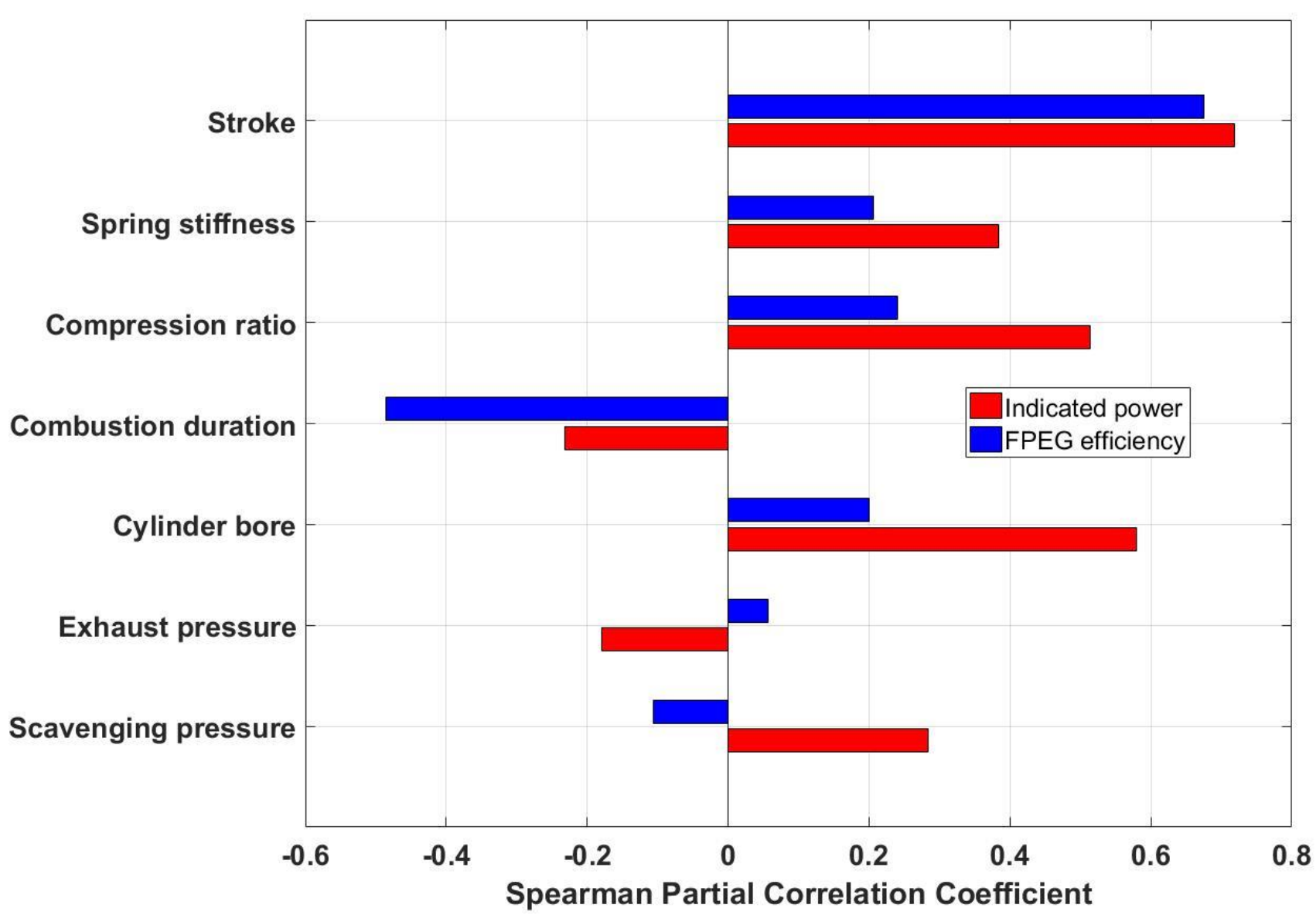

Figure 85: Spearman correlations for indicated power and system efficiency of an FPEG system with variable stroke length. 
Table 9: Range \& fundamental values of parameters for final global sensitivity study.

\begin{tabular}{|c|c|c|c|}
\hline Parameter & Range & Fundamental value & Units \\
\hline Cylinder bore & $30-46$ & 38 & $\mathrm{~mm}$ \\
\hline Effective stroke length & $26-36$ & 30 & $\mathrm{~mm}$ \\
\hline Scavenging pressure & $1.01-1.21$ & 1.10 & $\mathrm{bar}$ \\
\hline Exhaust pressure & $0.95-1.01$ & 0.99 & $\mathrm{bar}$ \\
\hline Combustion duration & $1.5-3.0$ & 2.25 & $\mathrm{~ms}$ \\
\hline Trapped compression ratio & $6-13$ & 8.25 & - \\
\hline Spring stiffness & $486-702$ & 558 & $\mathrm{kN} / \mathrm{m}$ \\
\hline
\end{tabular}

From Figure 85, the effective stroke was found to have a major effect compared to all the model inputs under constraint of their selected ranges. An increase in effective stroke increased both indicated power and efficiency. With an increase in the stroke length, the translator velocities were increased. Moreover, the translator covered more cylinder volume with increasing stroke length. As a result, the indicated work and power outputs were increased with increasing stroke length. Furthermore, an increase in the effective stroke length for the same cylinder bore reduced the cylinder heat transfer. This was due to a reduction in the surface area. In addition to this, the time available for the gas exchange was also increased with increased stroke length. As a result, the amount of fuel and air mixture delivered was increased. Altogether, the reduced heat transfer losses increased the FPEG efficiency with an increase in the stroke length. The variations in the LEM electrical power with an increase in effective stroke length are shown in Figure 86.

From Figure 86, the LEM power was increased almost linearly with the increasing stroke length. This was due to an increase in the translator velocities. When the effective stroke was increased from $26 \mathrm{~mm}$ to $34 \mathrm{~mm}$, the LEM power was increased from $400 \mathrm{~W}$ to $1200 \mathrm{~W}$. Moreover, a lot of interactions in the LEM power were observed at stroke lengths below $30 \mathrm{~mm}$. These interactions were attributed to the short gas exchange and port operation durations at stroke lengths below $30 \mathrm{~mm}$. As a result, the variations in the gas exchange and port operation durations were high. These high variations caused the interactions in LEM power. Finally, for stroke lengths above 30 $\mathrm{mm}$, the numerical model predicted an almost linear increase in the LEM power. This is evident from the congested numerical samples for the stroke lengths above $30 \mathrm{~mm}$. 


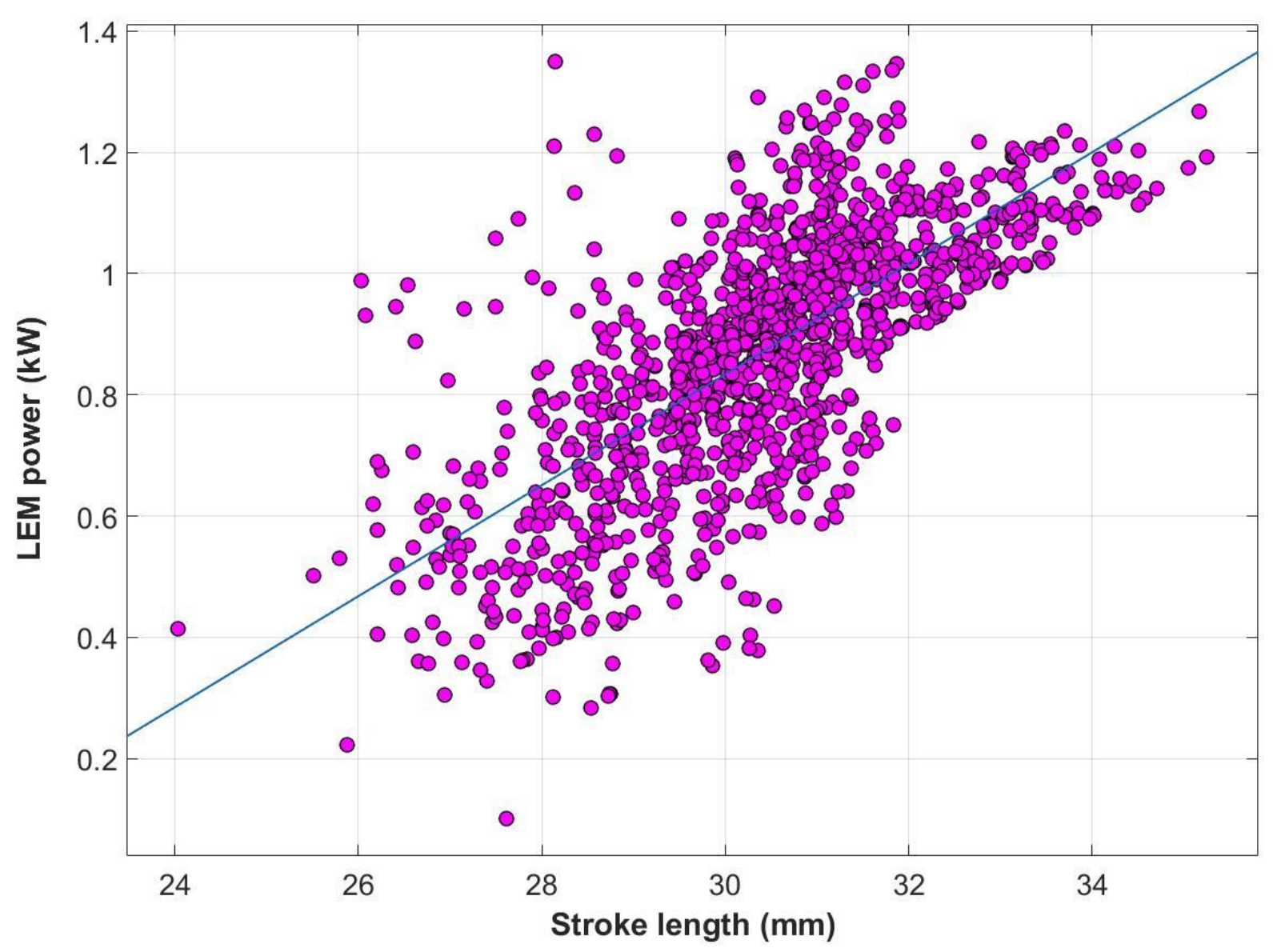

Figure 86: Variation in the LEM power output with the stroke length.

The next important model inputs were the cylinder bore and compression ratio. An increase in the cylinder bore increased the combustion cylinder forces and indicated work. This was due to an increase in the cylinder volume for a given stroke due to piston area. Moreover, with the increase in the compression ratio, the cylinder pressure was increased until the heat transfer losses curbed the further increase. The cylinder pressures further increased the indicated power output. After the bore and compression ratio, the spring stiffness was found to have the dominant effect on the indicated power. It is well known that increasing the spring stiffness increases the moving mass. The system frequency is dependent upon the relative increase in the ratio of spring stiffness to moving mass. In this investigation, the spring stiffness was varied from $486 \mathrm{kN} / \mathrm{m}$ to $702 \mathrm{kN} / \mathrm{m}$. Based on these values, the frequency was increased by $12 \mathrm{~Hz}$. This narrow frequency band was mainly responsible for the reduced spring stiffness dominance. The main reason for the narrow 
spring stiffness was due to the limitations of springs in the experimental system. The variation in the LEM power output with respect to frequency is shown in Figure 87. Unlike the distribution in Figure 86, the LEM power showed a lot of interactions with frequency. As a result, the Spearman coefficient was found to be less for the spring stiffness.

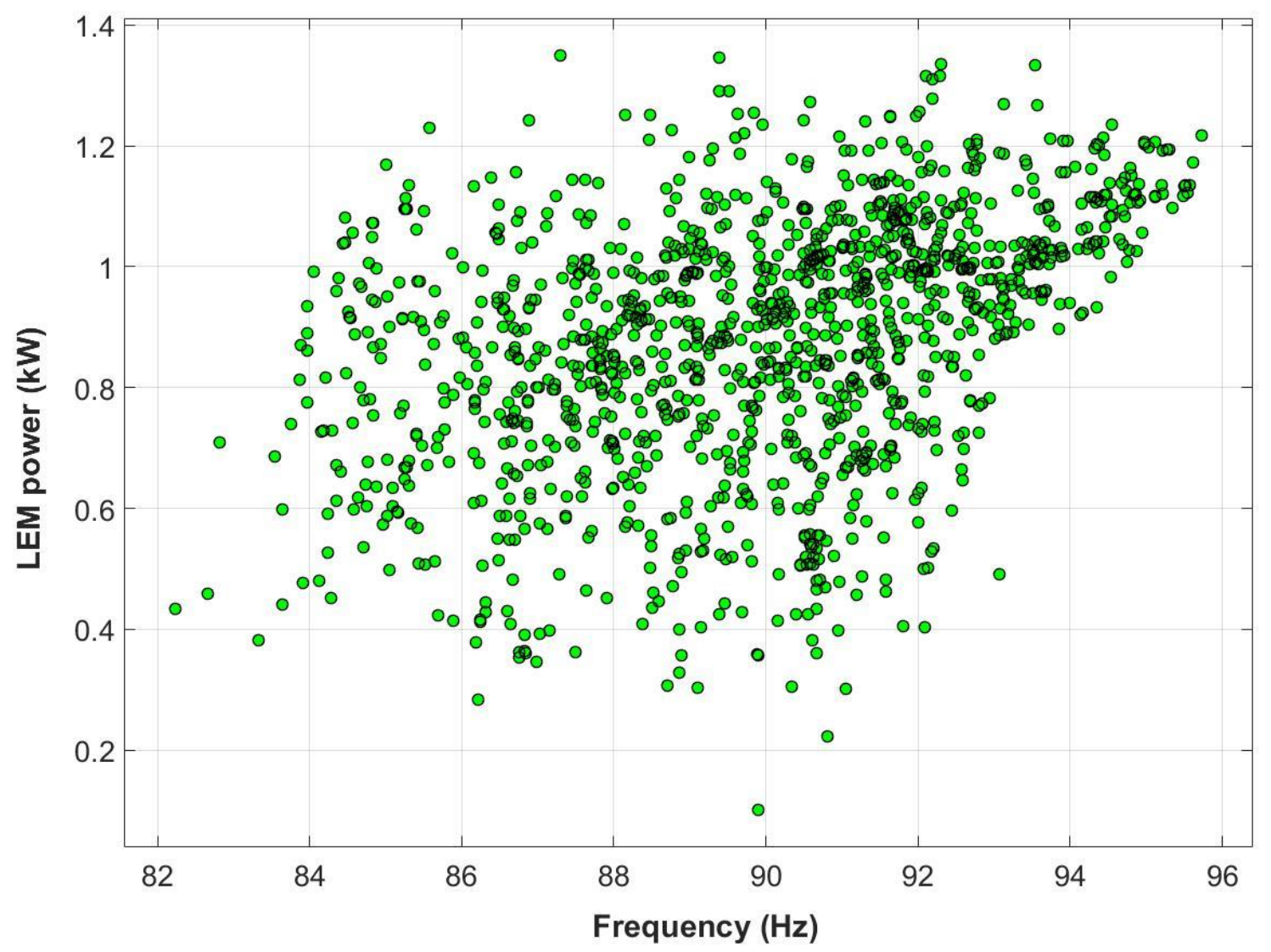

Figure 87: Variation in the LEM power output with operating frequency.

After spring stiffness, the scavenging pressures were found to have a major effect on the indicated power. Increasing the scavenging pressure effectively discharged the exhaust products. This effective discharge of exhaust products allowed more fuel and air mixture trapped inside the combustion chamber. With an increase in fuel and air mixture, the HRR was increased. This increased HRR was further responsible for an increase in indicated power. Moreover, the supercharging increased exhaust heat, friction, fuel slip, and conversion losses. These losses reduced the FPEG efficiency. On the whole, supercharging was used as an option to improve the 
indicated power. However, this supercharging was beneficial only if the FPEG efficiency was not the main objective.

On the negative side, increasing the exhaust pressure reduced the indicated power output. As the exhaust pressure was increased, the pressure gradient between the cylinder and exhaust was reduced. Moreover, the flow models in this investigation were dependent upon the pressure gradient. With the reduced pressure gradient, the exhaust discharged out of the system was reduced. In other words, the EGR in the cylinder was increased with increasing exhaust pressure. To maintain the stoichiometric condition, the controller reduced the amount of fuel delivered. This reduced fuel reduced the HRR and indicated power.

Finally, the combustion duration reduced the indicated power more dominantly than other model inputs. The primary reason for this decrease was the loss of fuel energy potential with delayed combustion. In addition to this, the exhaust heat losses were also increased with an increase in combustion duration. Moreover, the heat transfer and frictional losses were reduced with increasing combustion duration. However, the increase in the exhaust heat was higher than the reduction in heat transfer losses. As a result, the reduction in the FPEG efficiency was more than the indicated power.

On the whole, the stroke length was found to have a dominant effect on the indicated power when a variable FPEG system was considered. The stroke length was followed by cylinder bore, compression ratio, spring stiffness, scavenging pressure, combustion duration, and exhaust pressures. In terms of FPEG system efficiency, again the stroke length was found to be a dominant effect than other inputs. The stroke length was followed by combustion duration, compression ratio, cylinder bore, spring stiffness, scavenging pressure, and exhaust pressures.

\subsubsection{Fixed stroke length}

In the previous section, the stroke and compression ratio were varied in the broader range. Hence, these inputs were found to have a dominant effect on FPEG performance. In this section, a global sensitivity analysis study was performed with a constant effective stroke of $30 \mathrm{~mm}$. The other model inputs were changed in the same range as shown in Table 9. The only exception was the range of trapped compression ratio. The compression ratio was changed in-between 6 to 9 in this case. The reduced range incorporated the operating range of an experimental FPEG. Moreover, 
the fixed stroke was maintained by a dead center clearance setpoint controller. More details regarding the dead center clearance setpoint controller are found in Chapter 5 . With the fixed stroke and reduced compression ratio range, the model imitated the experimental FPEG system. The Spearman coefficients for model inputs with a fixed stroke of $30 \mathrm{~mm}$ are shown in Figure 88.

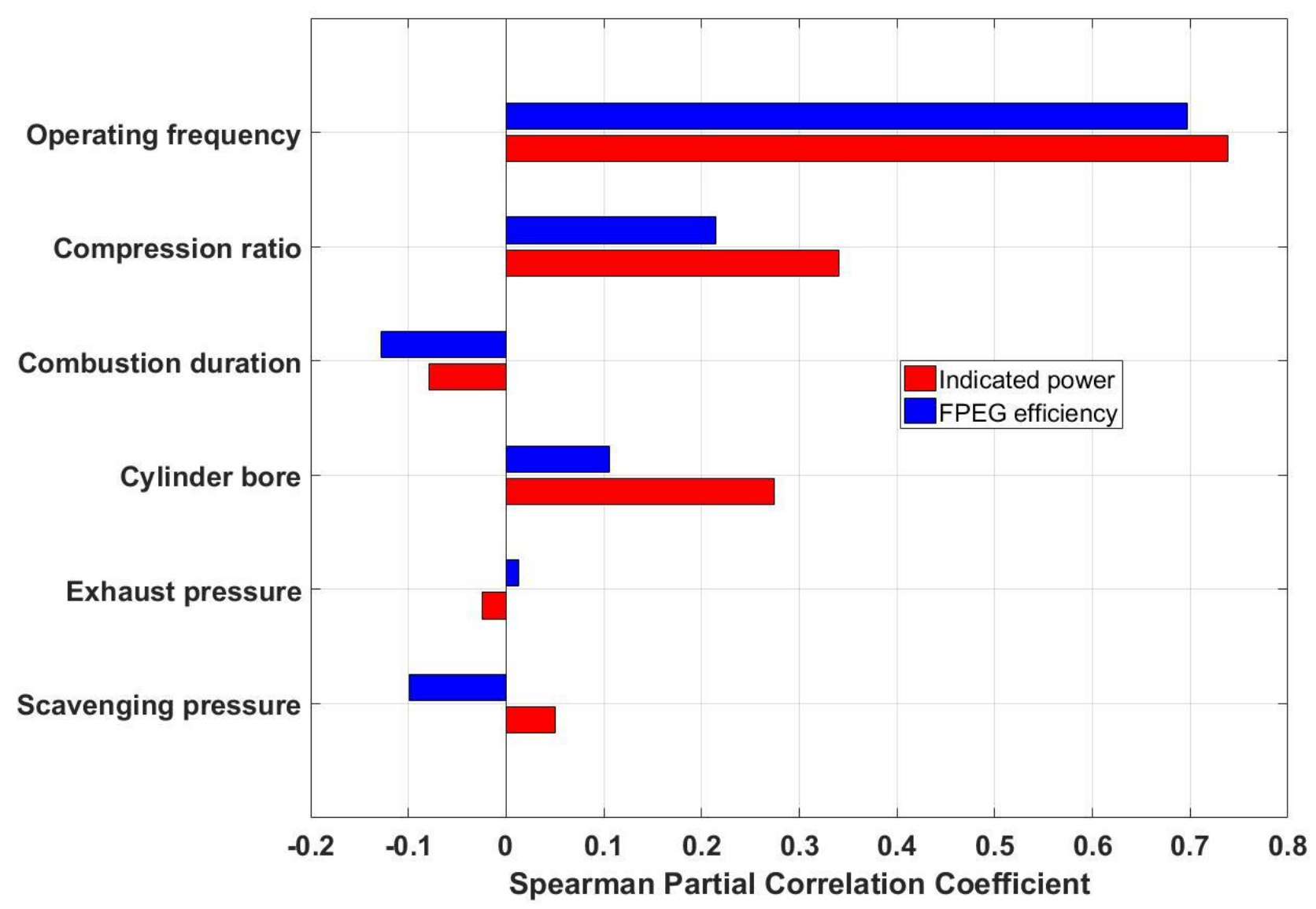

Figure 88: Spearman partial correlation coefficients for model inputs at a constant effective stroke of $30 \mathrm{~mm}$.

From Figure 88, the frequency defined by spring stiffness was found to have a dominant effect on the FPEG performance. One reason for the dominance of frequency was due to a reduction in the range of compression ratio. Moreover, the interactions induced by stroke were also eliminated in this case. The variable stroke length interactions increased the dominance of the cylinder bore and compression ratio in the results mentioned in Section 4.5.1. Since these interactions were eliminated, the Spearman correlations were reduced for the compression ratio and bore. However, the compression ratio and cylinder bore were found to be second and third dominant 
inputs after the frequency. Similar to Section 4.5.1 results, the combustion duration, scavenging and exhaust pressures were found to be the least dominant inputs. While increasing the scavenging pressure increased the indicated power, the increase in the combustion duration and exhaust pressures reduced the indicated power.

On the whole, of all the model inputs, the effective stroke length was the most dominant. This was because of its combined effect in improving the LEM and engine performance. The stroke length was followed by the cylinder bore, compression ratio, and spring stiffness. Of these four model inputs, three inputs were related to the system geometry. One of them was the operating variable, i.e. spring stiffness. Based on the results, it is evident that the key to the FPEG system performance is the FPEG geometry itself. The sensitivity analysis results are employed in the further improvement of the experimental FPEG system. 


\section{FPEG CONTROL ARCHITECTURE}

In CSE systems with the crankshaft mechanism, the rotating speed is controlled by two methods. The first method uses an electronic system with a trigger or position sensor. The second method uses a mechanical system employing centrifugal force. In either method, the controller's responsibility is to match the engine power to the load demand. Without such a controller, the CSE still finds a steady operating rotating speed. However, the rotating speed of an uncontrolled system is decided by the non-linear nature of the friction or engine load. This rotating speed usually does not correspond to the target rotating speed. With an extreme mismatch of energy demand and supply, the engine is either runs at an over speed or stall. This is because of a cyclic net addition or subtraction of energy to the flywheel. In other words, the quest is for cycle-to-cycle constant energy production represented by a constant flywheel rotational speed when used with a steady load.

The FPEG systems without springs naturally run in a limited frequency band. With the inclusion of stiff springs, the frequency band turns out to be even tighter when compared to the design without springs. As a result, the FPEG cannot store substantially more or less energy without changing its frequency and stroke. If the LEM load consumption is greater than the engine combustion output, then the stroke length, peak cylinder pressure, the velocity at mid-stroke will reduce on the following cycles. As a result, the system will "ring down" to a stall. If the engine combustion delivers too much energy, this energy leads to increased gas pressure energy with an increased stroke and compression ratio. At mid-stroke, this same combustion energy is assigned to high translator velocity associated with an increase in stroke length. In the end, the heat transfer, ring friction, and blowby losses also consume excess engine power. However, this operating condition is unlikely to be optimal or efficient. As a result, the system is subjected to mechanical damage. This is similar to the CSE system operating at too high a speed. Therefore, it is essential to manage the energy in the FPEG system. In other words, matching the engine output to the generator demand.

The FPEG energy management is obtained by utilizing an energy balance control algorithm. This transient control algorithm is similar to the use of a governor and flywheel in a CSE system. In a CSE, the rotational speed changes with the constant compression ratio. However, in an FPEG system, the goal is to regulate the piston dynamics at a constant frequency. Therefore, it is important to monitor the FPEG system energy from cycle-to-cycle. For modeling purposes, one 
may regulate the FPEG system's energy balance by managing either engine output or generator demand, or both. From a MATLAB®/Simulink modeling perspective either is acceptable to achieve the steady state operation. In this dissertation, the energy balance controller was implemented to modify the LEM demand for different operating conditions. The key motivation for considering the LEM load was to build a CHP system functioning at a near-constant load of $1 \mathrm{~kW}$. Moreover, the throttling of the engine created a substantial power loss. In other words, the throttling impacted the spring forces as well as the energy output. Considering these reasons, the controller's aim was to modulate the LEM load at a wide-open throttle condition.

In addition to this energy management controller, other controls were also required for the steady FPEG operation. One of the controls was the control of the LEM operating modes. Depending upon the system energy, the LEM was operated as a motor and a generator. During the startup, the LEM was operated as a motor till the combustion favorable conditions were met. The combustion favorable conditions were defined based on compression ratio and port locations. After reaching the combustion favorable conditions, the LEM was switched to the generator mode. In case of ring-down or stall in the generating process, the LEM was switched back to the motoring mode again. Besides these, controls were also required for MBT timing, ignition signals, and injector parameters. To achieve stable FPEG operation, a model-based multi-level control strategy was developed in MATLAB®/Simulink. This chapter deals with the development of a model-based multi-level control strategy. Especially during the generating phase, a controller with six levels was proposed in the numerical model. The levels include model-based calibration maps, energy management, MBT timing, ignition signals, injection pulse width, and injection signals. The submodel of the controller at its highest level is shown in Figure 89.

The submodel received the information of compression ratio, translator dynamics, location of ports, combustion favorable compression ratio, exhaust composition, equivalence ratio, MFB, and dead center locations. Based on these inputs, the multi-level controller provided LEM and engine modes, MBT timing, ignition signals, injector parameters, generator gain, and motoring frequency as outputs for the plant system. The individual submodels for these six levels and the calculation procedure of these outputs were provided in the upcoming sections. 


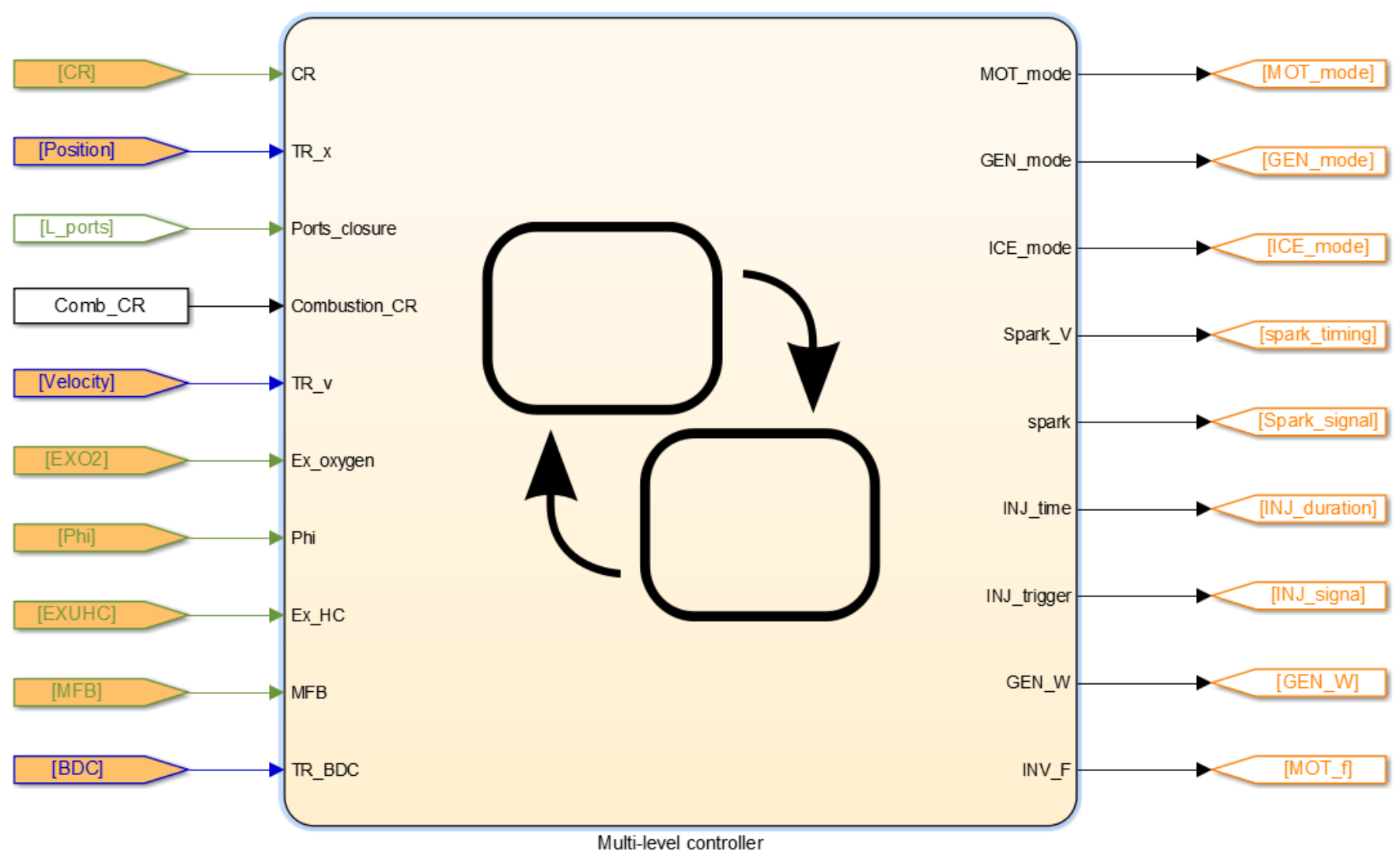

Figure 89: Submodel of a multi-level controller developed in MATLAB®/Simulink.

\subsection{LEM mode switch controller}

This section reported the control logic related to the LEM switching between motor and generator modes. Initially, the LEM was operated as a motor until the combustion favorable conditions of ICE were met. The combustion favorable conditions were defined by prescribing a target trapped compression ratio. The compression ratio was used as a combustion favorable condition due to the measurement of experimental FPEG dynamics. As a result, the compression ratio was calculated in real time. In other words, this control algorithm was developed with an intent of employing it in the experimental prototype. If the actual FPEG compression ratio exceeded the target trapped compression ratio, the LEM was switched from motor to generator mode. The control logic of switching between the LEM operating modes is shown in Figure 90.

In Figure 90, the arrows represented the transition from one state to another state. By default, initially, the controller entered the motoring phase. Thus, the LEM was operated as a motor. This 
was evident from the value of '1' assigned to the 'MOT_mode' variable. Moreover, the motoring and generating modes (i.e. 'MOT_mode' and 'GEN_mode') were the two exclusive states of the same LEM. These exclusive states were never activated or executed at the same time. In other words, the variable 'MOT_mode', 'GEN_mode' cannot be equal to 1 at the same time. Due to this condition, the variable 'GEN_mode' was set to zero. Moreover, the cylinder conditions were not favorable to combustion during the motoring mode. Therefore, the engine was also turned off. When the engine was turned off, no fuel was introduced into the combustion chamber.

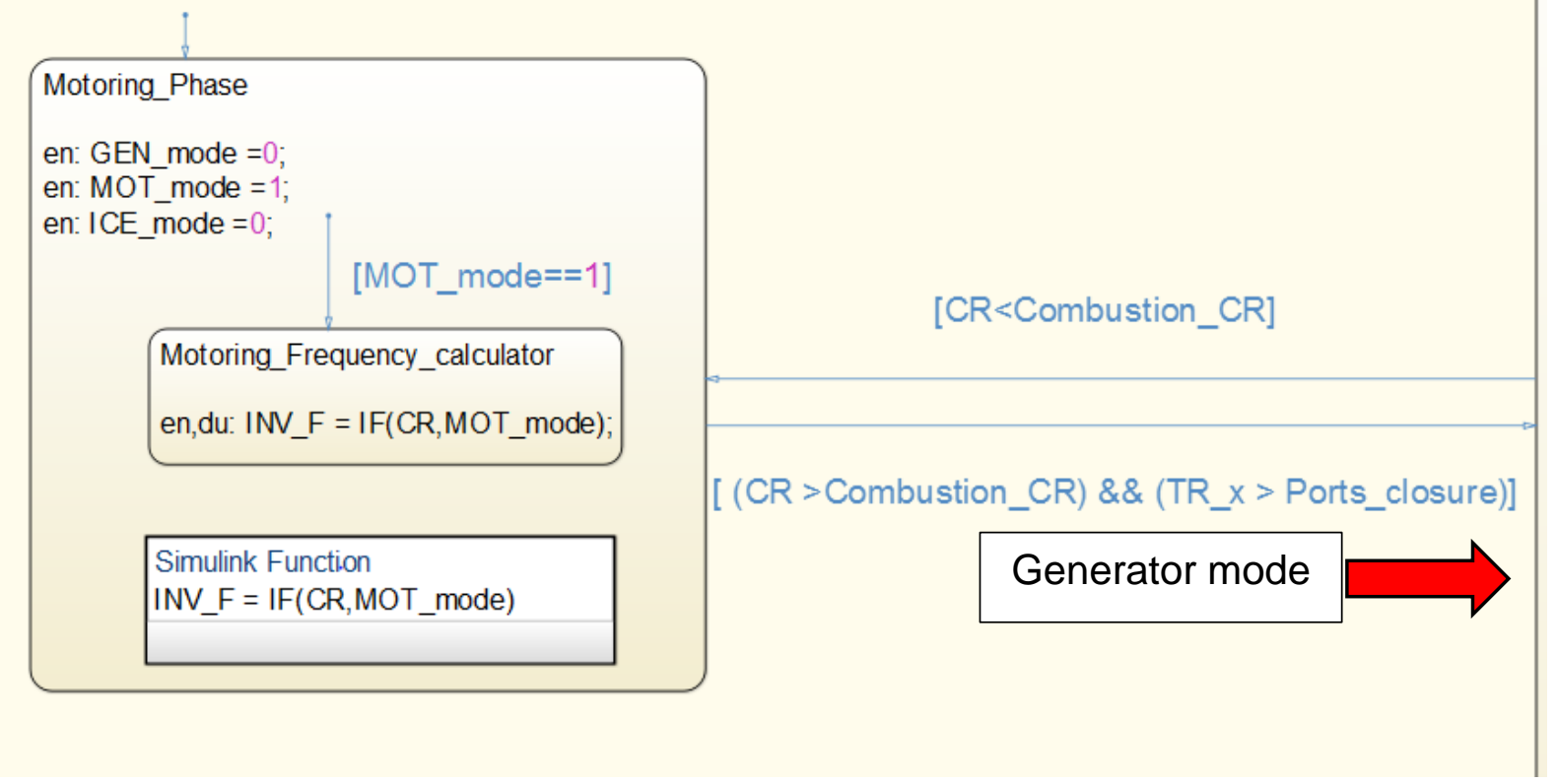

Figure 90: Submodel of a LEM inverter model developed in MATLAB®/Simulink.

From Figure 90, it was evident that the motoring mode had two transitions defined by the blue signal arrows. One transition was switching from the motoring phase to the generating phase. This transition was attained only if the following two conditions were met. The first condition was the displacement exceeding the EPC location. The second condition was the actual compression ratio exceeding the combustion-favorable compression ratio. This combustion-favorable compression ratio was provided as an input to the numerical model. If these two requirements were true, then the LEM was switched from motoring to generating mode. Similarly, if the actual compression ratio was less than the combustion-favorable compression ratio, then the LEM was switched back to the motoring mode. These control logics were shown above and below the blue 
arrows in Figure 90. In addition to these modes, an additional neutral mode can also be added to the current control architecture. This neutral model represents the variable generator load. However, the controller in this dissertation was limited to only two operating modes.

When the motoring phase was active, the controller provided resonant frequency as the output. The resonant frequency was calculated by applying the Phase Lock Loop (PLL) to the translator velocity. Later, this frequency was used to calculate the location and width of the motoring pulse. As mentioned in Chapter 2, an $\mathrm{H}$-bridge circuit was designed for the operation of the LEM as a motor. The H-bridge circuit consisted of four IGBT's systems arranged in two legs. Since the FPEG system employed stiff springs, only two IGBT's were operated at any time. The return thrust force was supplied by the springs under the expansion process. The translator Stateflow ${ }^{\circledR}$ diagram for the calculation of piston reversal and dead center locations is shown in Figure 91. This diagram calculated the TDC, BDC locations, and frequency based on the sign change in translator velocity.

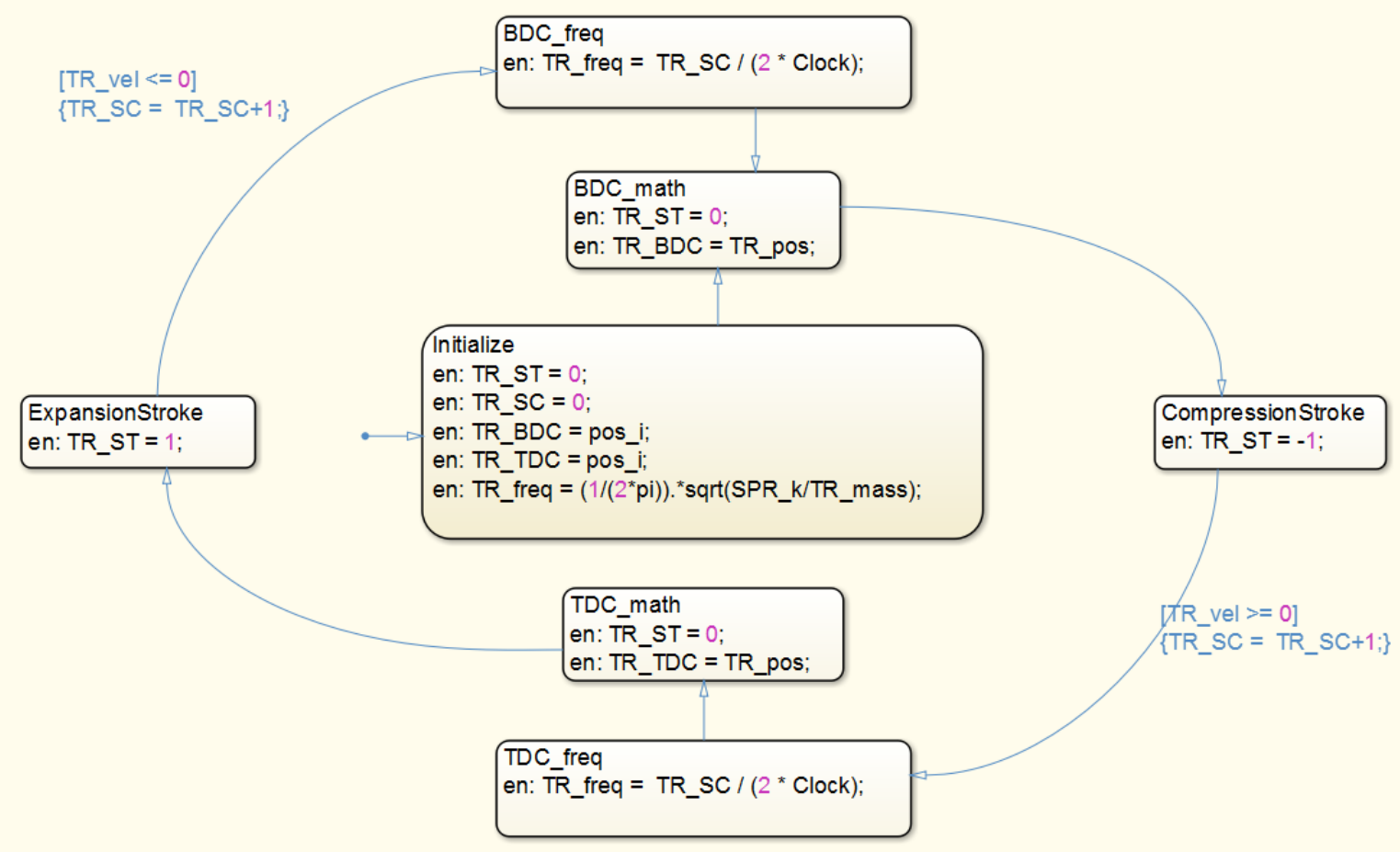

Figure 91: Calculation of resonant frequency based on piston reversal. 
From Figure 91, initially, the FPEG was started from rest. This rest position was assumed as BDC. The BDC location was calculated by using a 'BDC_math' state representation block. After, the index ('TR_ST') for the stroke direction (i.e. compression or expansion stroke) was calculated. The indexes of ' -1 ' \& ' +1 ' corresponded to the compression and expansion strokes respectively. In other words, the translator velocity was assumed to be negative for the FPEG compression stroke. Once the translator velocity became positive, the controller calculated the resonant frequency. This value was obtained based on the time period between two consecutive velocity sign changes. After calculating the resonant frequency, the controller calculated the TDC position and changed the stroke index. Once the controller detected the negative velocity, the resonant frequency, and BDC locations were calculated. Finally, the frequency and dead center locations were used in the engine cylinder model for defining the ignition and injection parameters.

\subsection{Hierarchical generator controller}

In the generating phase, the controller should manage the system energy along with providing the ignition and injection controls. The submodel of the generating phase controller is shown in Figure 92. During the generating mode, the generator and engine systems were activated. This was evident from the GEN_mode and ICE_mode parameter values. In the motoring phase, the functional block of motoring frequency was represented by a solid line. In the generating phase, the functional blocks were represented by dashed lines. The solid lines represented the series or exclusive operating modes. The dashed lines represented the parallel or independent operating modes. In a parallel operating mode, two or more states were active at any instant. For example, the model-based calibration map and energy management subsystems were active at the same time. In a series operating mode, only one functional block was active at any instant. In addition, a number was assigned on the top right for each functional block in this mode. These numbers represented the order of execution. In Figure 92, the model-based calibration maps block was given the first preference. It was followed by the energy management, ignition, and injection blocks. If the compression ratio was below the target value, the LEM was switched back to the motoring phase. Moreover, the ignition and injection systems were also turned off due to this switching. More details about various control blocks in the generator phase are explained below. 


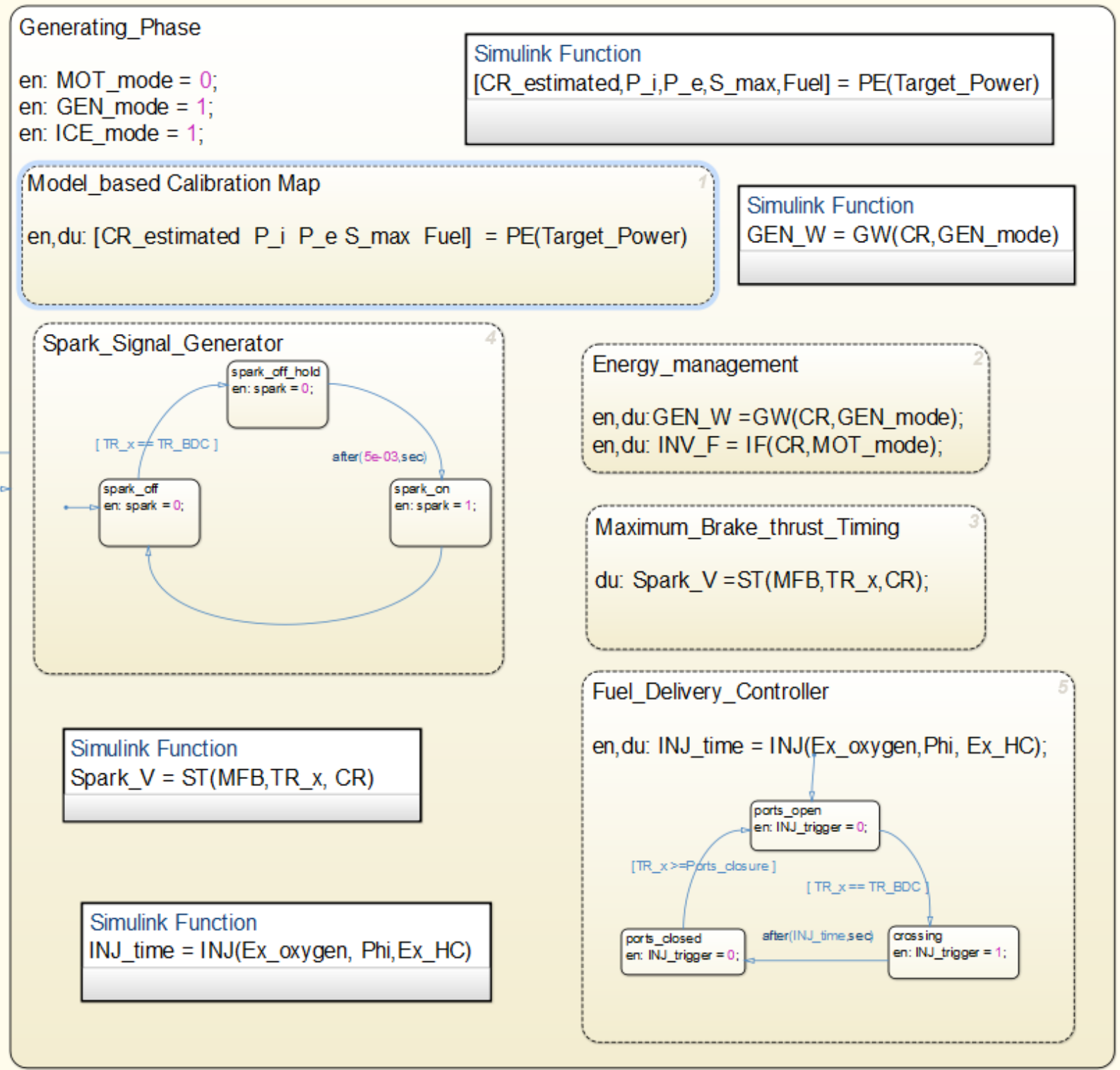

Figure 92: Generating phase controller submodel developed in MATLAB®/Simulink.

\subsection{Model-based calibration map controller}

The main purpose of the model-based calibration map controller was to act as a supervisory controller. This controller provided the target FPEG operating parameters for a given power output. The sensitivity analysis results from Chapter 4 were used to attain these maps. In an SI engine, the indicated power was controlled by controlling the throttle, exhaust pressure, and fuel. 
In CSE systems, the indicated power was also controlled by changing the speed along with those variables. Since the FPEG system used stiff springs, it operated in a narrow frequency band. Hence, the only way to control the FPEG indicated power was to change the operating conditions. The operating conditions included the air induced into the system using the throttle, exhaust pressure, fuel, and ignition timings. Moreover, the variable stroke length and compression ratio were also considered. The model-based calibration maps played an essential role during the operation of FPEG plant and controller models. These maps were provided to the controller as two-dimensional lookup tables. The model-based calibration maps for various parameters are shown in Figures 93 to 95.

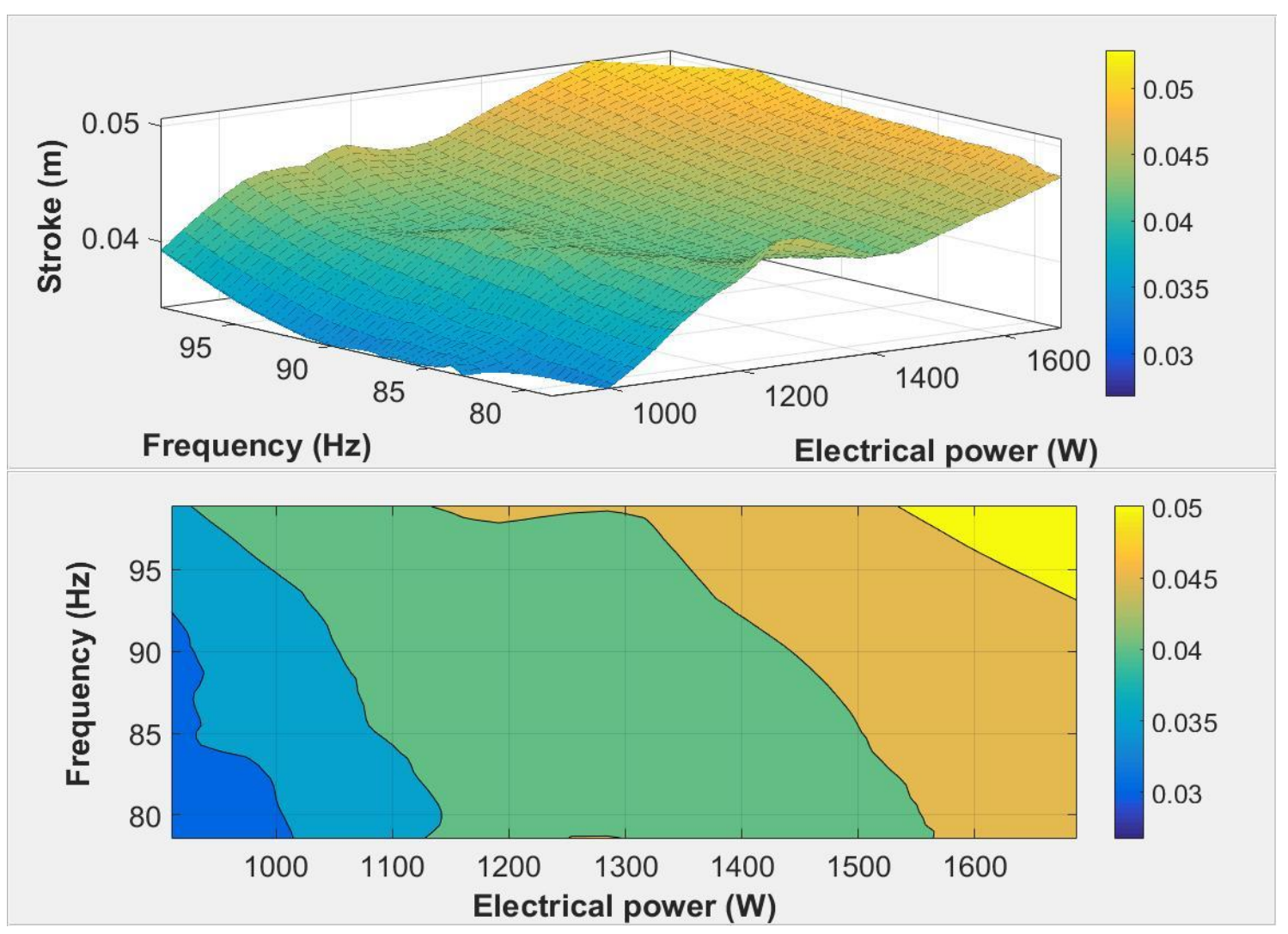

Figure 93: Model-based calibration map of stroke length for target electrical power and frequency.

Initially, the LEM power demand was provided to the numerical model. For example, the LEM demand was set to $1 \mathrm{~kW}$ for the modeled FPEG system. There was no restriction on the LEM demand. Due to the DOE ARPA-E targets, the LEM electric power was set to a fixed value rather than a variable. 


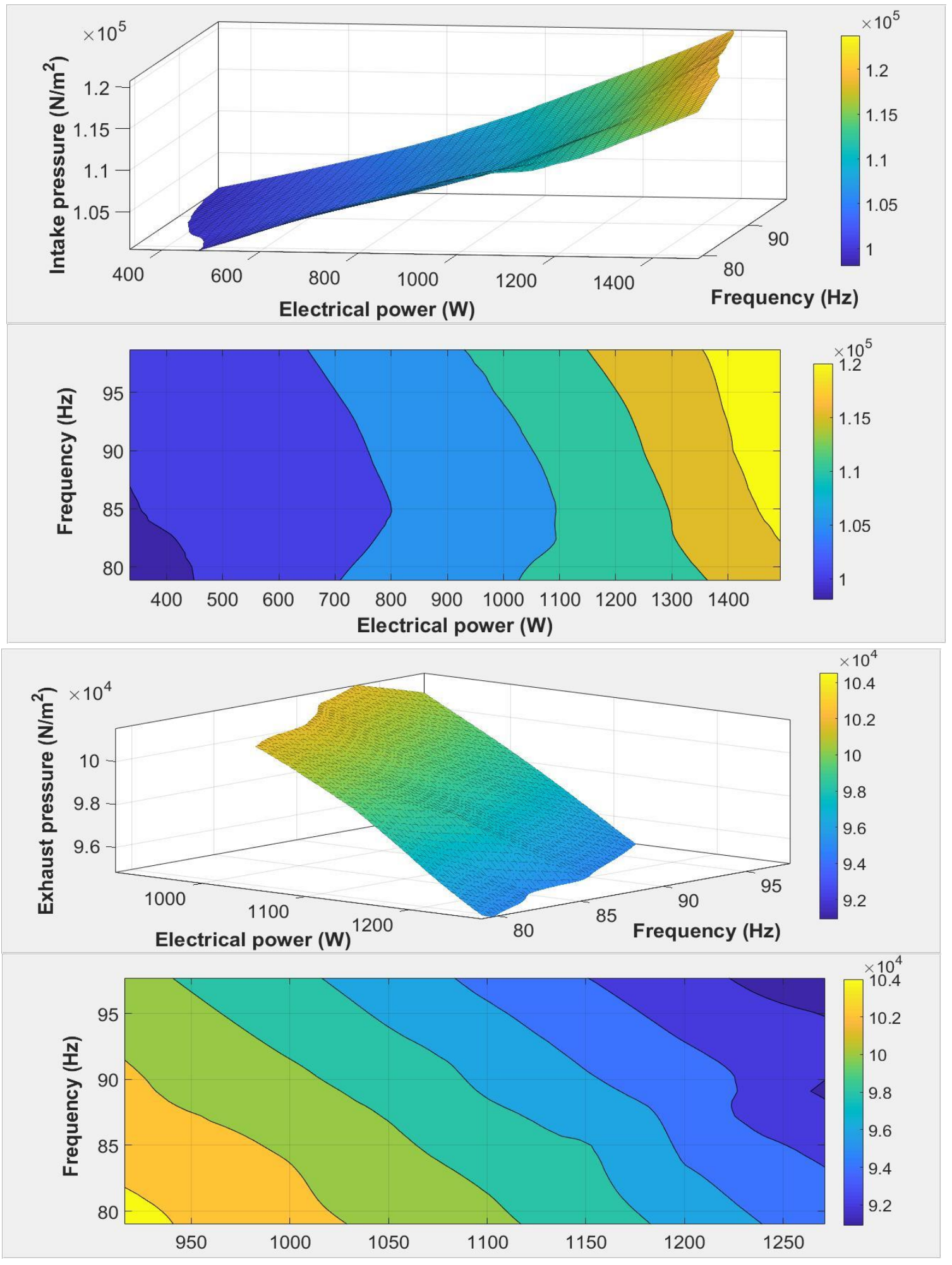

Figure 94: Model-based calibration map of intake and exhaust pressures for target electrical power and frequency. 
The next important variable was the system frequency. The LEM demand and frequency were considered as the base parameters. The model-based controller map provided the outputs based on the base parameters. The outputs were the target compression ratio, stroke length, scavenging, and exhaust pressures. While developing these calibration maps, the ignition timing was fixed to the MBT timing. In other words, the FPEG was operated at an optimized ignition timing for all the conditions. The fuel in this investigation was considered by means of equivalence ratio. Based on the target equivalence ratio and EGR, the controller regulated the fuel.

With the change in the LEM demand, the target operating parameters were updated at each time step. Later, they were provided as inputs to the energy management controller and FPEG plant systems in the next level.

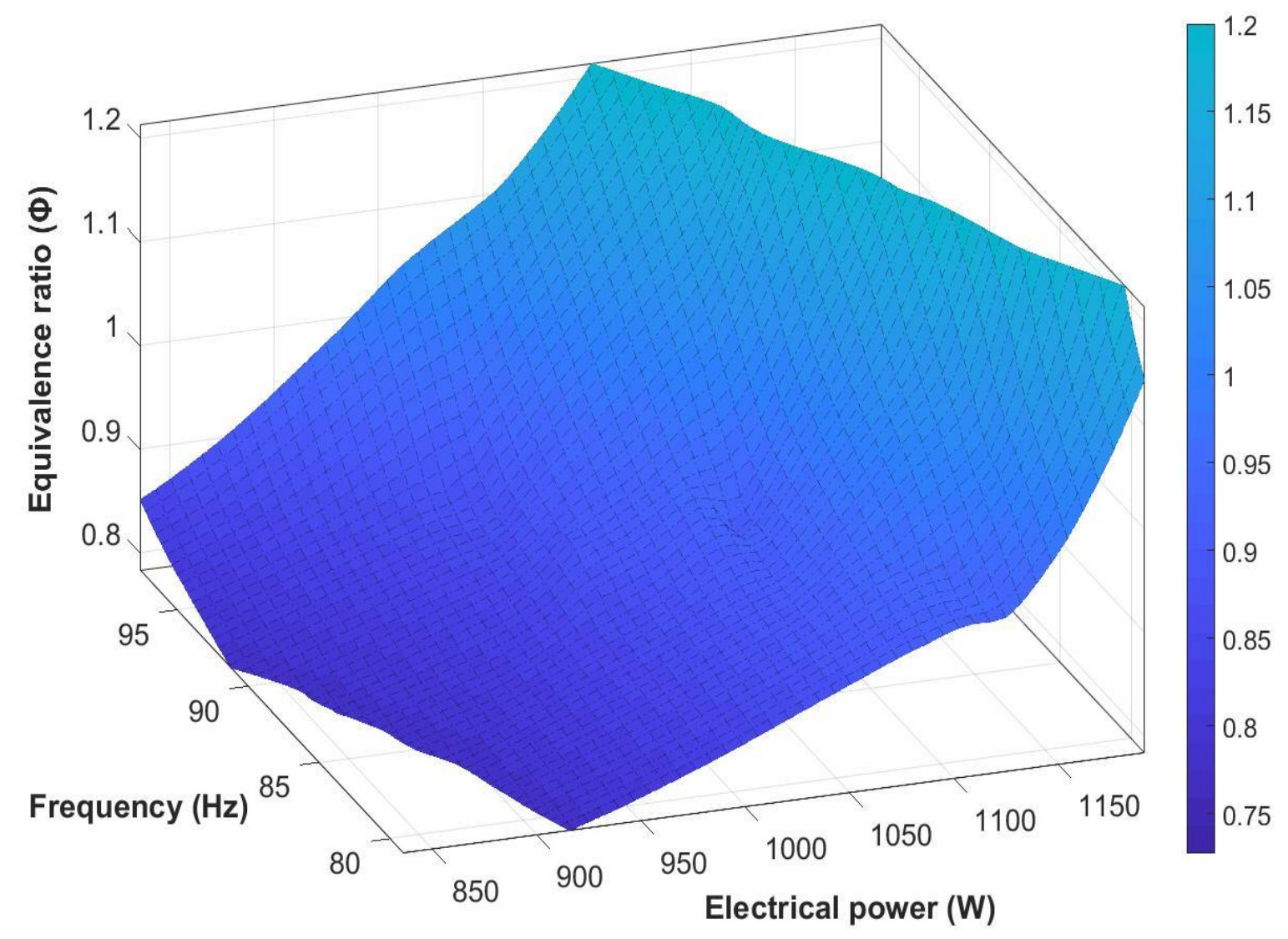

Figure 95: Model-based calibration map of equivalence ratio for target electrical power and frequency. 


\subsection{Energy management controller}

As mentioned in the introduction, the energy management from ICE to LEM demand was important. Without the energy management controller, the FPEG system either ramped up, misfired or ramped down to a stall. This ramp up and ramp down FPEG operation was unlikely to be optimal. Moreover, the operation was defined by the losses in the system. In this section, an energy management controller was modeled for stable FPEG operation. Initially, the FPEG system was made to operate without an energy management controller. This study highlighted the importance and the need for an external controller for stable FPEG operation.

During this study, the LEM power was modeled as a product of translator velocity and LEM load coefficient (gain). The LEM load coefficient scaled the LEM energy according to the engine output in one cycle. In other words, increasing the load coefficient increased LEM power. Figure 96 shows the peak pressure and mid-stroke velocity variations for different LEM load coefficients.

In Figure 96, each line represents the FPEG system behavior for a given LEM load coefficient. A difficulty during the numerical modeling was associated with the FPEG cycle-to-cycle energy balance. The same difficulty was reflected in Figure 96 . For a LEM coefficient of $15 \mathrm{~N}-\mathrm{s} / \mathrm{m}$, the LEM consumed less power than the engine power generated in one cycle. As a result, the peak pressures and the compression ratio were increased from cycle-to-cycle. This process was repeated until the heat transfer losses curbed the further increase. The uneven peak pressure distribution in Figure 96 was mainly due to the cycle-to-cycle variations in various submodel operations.

For a LEM coefficient of $26 \mathrm{~N}-\mathrm{s} / \mathrm{m}$, the LEM consumed more energy per cycle than the engine output generated in one cycle. Consequently, the compression ratio and stroke length decreased gradually from cycle-to-cycle. The reduction in the compression ratio and stroke length further reduced the peak cylinder pressures. The green line in the center of Figure 96 showed the FPEG operation with an external controller. This was represented as '22WC' in the legend in Figure 96. In this case, the LEM load coefficient was varied with respect to the error in the trapped compression ratio. 

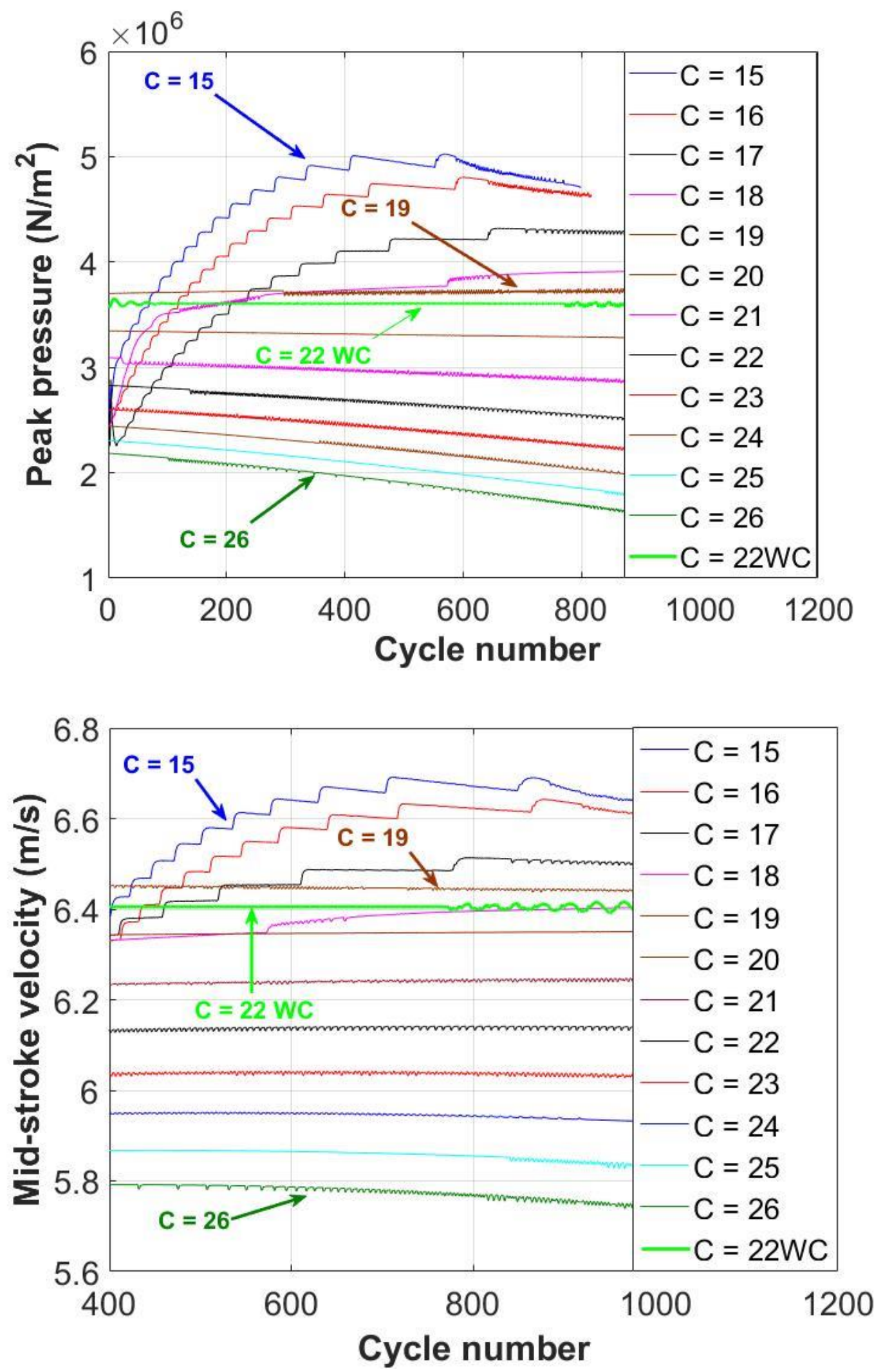

Figure 96: Variations in peak pressure and mid-stroke velocity of the FPEG for different LEM load coefficients. 
Due to the external controller, the FPEG system operated steadily with minimal cycle-to-cycle variations. Moreover, the average load coefficient value was found to be $19.45 \mathrm{~N}-\mathrm{s} / \mathrm{m}$ with an external controller. From Figure 96, for a LEM coefficient of $19 \mathrm{~N}-\mathrm{s} / \mathrm{m}$, the peak pressures almost remained constant with minimal cycle-to-cycle variations. With the peak pressure reduction, the indicated work was also reduced. This reduction in the indicated work further resulted in the efficiency reduction. The same trend was observed in the mid-stroke velocities for different load coefficients as shown in Figure 96.

One interesting feature observed in this study was the variation in the EGR with the LEM load coefficient. The EGR was calculated based upon the mass management submodel. The mass flow rate model was developed based on the perfect gas mixing model. As the load coefficient was increased from $15 \mathrm{~N}$-s/m to $26 \mathrm{~N}$-s/m, the EGR percentage was increased from $32 \%$ to $43 \%$. As the load coefficient was increased, the pressure gradient between the EPO cylinder pressure and exhaust pressure was reduced. The pressure gradient was mainly responsible to the reduction in the cylinder pressure on successive cycles. These pressure variations increased the EGR as the load coefficient was increased. Moreover, the compression ratio and mid-stroke velocity reduction in successive cycles also opened the exhaust port for a long time. Finally, from Figure 96, the FPEG cycle-to-cycle variations were high without an external controller. This was due to the net cyclic addition and subtraction of energy in the system. These cycle-to-cycle variations eventually stalled the system. Hence, the FPEG system needed an external controller to manage the cycle-to-cycle energy within the system.

\subsubsection{Energy management controller objectives}

Due to the absence of the crankshaft mechanism, the instantaneous summation of the forces defined the FPEG piston dynamics. The force deviations between consecutive cycles resulted in a variable compression ratio and variable stroke length. In an FPEG design, either the peak pressure, mid-stroke velocity, compression ratio, or dead center clearance set points were considered as the control variables. In a CSE, these variables were fixed parameters. If these control variables were increased on consecutive cycles, too much power was produced by the engine. If these control variables were decreased on consecutive cycles, the FPEG system moved towards a stall. Moreover, the actions of ignition and injection controls were dependent upon energy management control. The current section concentrated only on the energy 
management control for stable FPEG operation. Therefore, the main intentions of the FPEG energy management control system are:

1. The energy generated from the engine must be closely equivalent to the LEM energy demand every cycle.

2. The proposed control system should control either the compression ratio, dead center set points, peak pressures, or mid-stroke velocities independently. These variables must be controlled within tight tolerances at a substantially constant frequency.

3. The proposed energy balance control system should prevent excessive cylinder pressure in the system. Moreover, it should ensure the proper scavenging process during the transition from starting to generating mode.

In the CSE system, the crankshaft and connecting rod mechanism take care of these objectives. In an FPEG system, an energy management controller was used to achieve these objectives. Moreover, the proposed control systems were devised only at the modeling level. The hardware applications of the strategies were beyond the scope of the current research investigation. However, the controller was modeled with an intention of using it as the pathway for improving and optimizing the experimental FPEG design.

In this dissertation, compression ratio, dead center clearance set points, mid-stroke velocity, and maximum cylinder pressure were considered as the control variables. The target values for these control variables were selected based upon the experimental FPEG system. The target values were maintained constant throughout the dissertation. Moreover, the increase in these target values increased the FPEG efficiency and power output. However, varying the target values was beyond the scope of this dissertation. Initially, the numerical model was solved for the system of forces acting on the translator rod. Later, the control variables were calculated based on piston dynamics. These control variables were further evaluated in the energy management controllers. When the energy management controller detected the deviation in the target values, the controllers corrected the LEM load generating coefficient. The updated load coefficient was then used to calculate the LEM force. Finally, the corrected piston dynamics were attained based on the updated LEM force. 
In this dissertation, the LEM load was considered as an output variable. The reason for considering the LEM load was to build a CHP system functioning at a near-constant load of $1 \mathrm{~kW}$. Moreover, the throttling of the engine created substantial inefficiency. The engine forces further impacted the compression spring forces. Owing to these reasons, the controller's aim was to modulate the LEM load at a wide-open throttle condition.

\subsubsection{Energy management controller configurations}

In this investigation, two different controller architectures (i.e. PID and PDF) were considered at the modeling level. The architectures of these controllers are shown in Figure 97. These controllers were selected due to their widespread applications and performance characteristics. The PID controller, as shown in Figure 97, continuously analyzed an error value. The error was calculated based on the difference between the measured process variable (MPV) and the target setpoint (TSP). After error calculations, the correction was applied using the summation of proportional, integral and derivative terms. The control function of the PID feedback mechanism is expressed mathematically in Eq. 1.

$$
\begin{gathered}
C(t)=K_{p} e(t)+K_{i} \int_{0}^{t} e\left(t^{\prime}\right) d t^{\prime}+K_{d} \frac{d e(t)}{d t} \\
e(t)=T S P-M P V
\end{gathered}
$$

Eq. 1

In Eq. 1, $C(t)$ is the correction applied to the LEM load based on the error term. The term $e(t)$ is the continuously calculated error term. The terms $K_{p}, K_{i}, K_{d}$ are proportional, integral, and differential term constants respectively. The controller coefficients were optimized for both the controllers presented in this dissertation. The target compression ratio was set to 8.25. This value was calculated from the experimental FPEG system. The dead center clearance setpoints were selected based on the compression ratio and an effective stroke of $28.75 \mathrm{~mm}$. The effective stroke of $28.75 \mathrm{~mm}$ was selected because of the effective stroke of an experimental FPEG system. For the peak pressure input, the maximum pressures were maintained to be 35 bar. 

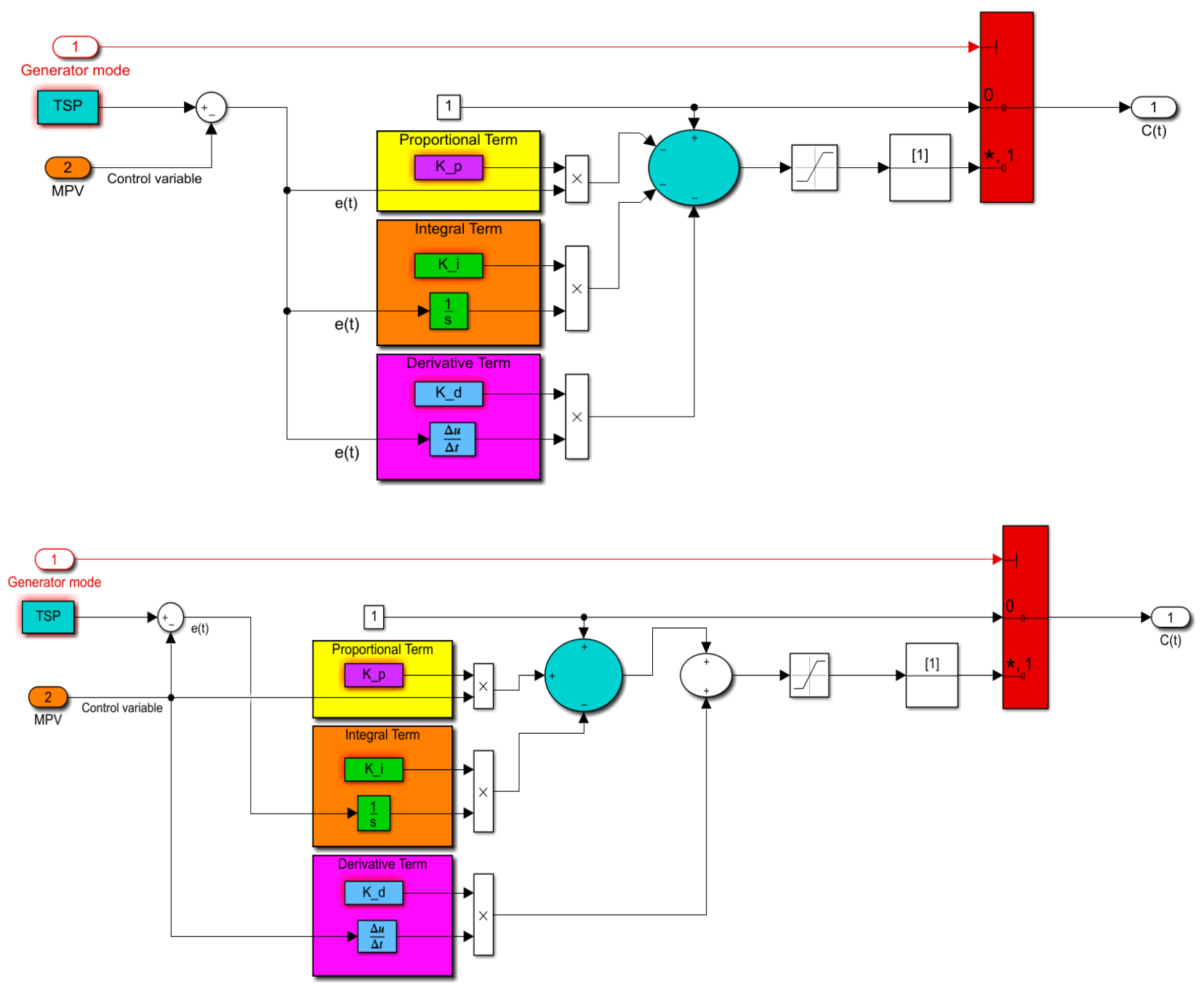

Figure 97: Control architecture of PID \& PDF controllers modeled in MATLAB®/Simulink.

In contrast, the PDF controller generally has better load management capabilities when compared to the PID controller [100]. However, the PID controller has a good target setpoint tracking ability. In the FPEG system, the rapid load changes pose the greatest challenge. The control function of the PDF controller is expressed mathematically in Eq. 2.

$$
C(t)=-K_{p} * M P V-K_{d} * M P V+k_{i} \int_{0}^{t} e\left(t^{\prime}\right) d t^{\prime}
$$

Eq. 2 
The PDF controller was not very responsive to the rapid target setpoint changes compared to the PID controller [95]. Owing to this advantage, the PDF controller was more suitable in the FPEG system with rapid load changes. The non-responsiveness of the PDF controller was due to the lack of derivative or proportional terms in the controller forward path. Therefore, the target setpoint variations of the PDF were much lower than the PID controller with optimized coefficients. By employing these controllers and stiff springs, the FPEG system behaved similarly to the CSE with substantially constant frequency and stable piston dynamics. The energy management controller behavior with the four control inputs were presented in the sections below.

\subsubsection{Controller simulation - Trapped compression ratio input}

In this section, the transient FPEG system response was investigated with trapped compression ratio as an input. The corresponding proportional, integral, and differential coefficients were tuned to optimized conditions. The optimal condition referred to minimum system settling time from these controllers. Moreover, only the compression ratio was controlled. In other words, the controller prevented excessive pressures in the combustion chamber. However, the stroke length was still variable. This was due to the lack of controller action at BDC.

Figure 98 shows the variation in compression ratio and TDC setpoint during the transient FPEG operation. Based on the modeling results, the PID and PDF controllers behaved in the same way in controlling the compression ratio. These two controllers prevented the compression ratio from reaching a high value during the transient mode. Moreover, a higher compression ratio was observed for the PDF controller when compared to the PID controller. This was due to the differences in the gain term action in these controllers. Moreover, only the integral term was used in the direct path of the controller in the PDF. As a result, the non-responsiveness of the PDF controller resulted in a higher compression ratio peak than the PID controller. In summary, both the controllers took rapid action in controlling the compression ratio. The target value was reached within 5 cycles after detecting the deviation in the compression ratio.

From Figure 98, during the first few cycles, the compression ratio was below the target setpoint. This was also evident from the positive deviation in the TDC setpoint. The gradual increase in the compression ratio in the initial cycles represented the resonance starting process. Once the combustion favorable conditions were reached, the deviation in the TDC position was reduced. As mentioned earlier, these combustion favorable conditions were determined in terms of a target 
compression ratio and port locations. Once the actual compression ratio was higher than the target value, then the mixture was ignited. Figure 99 shows the variations in the in-cylinder pressures and LEM power for both the controllers. The LEM power distribution in Figure 99 was obtained from a PID controller operation. The gradual increase in the cylinder pressures in the beginning was due to the mechanical resonance starting process. Due to resonance process, the pressures, the effective stroke length, and the compression ratio were gradually increased from cycle-to-cycle.
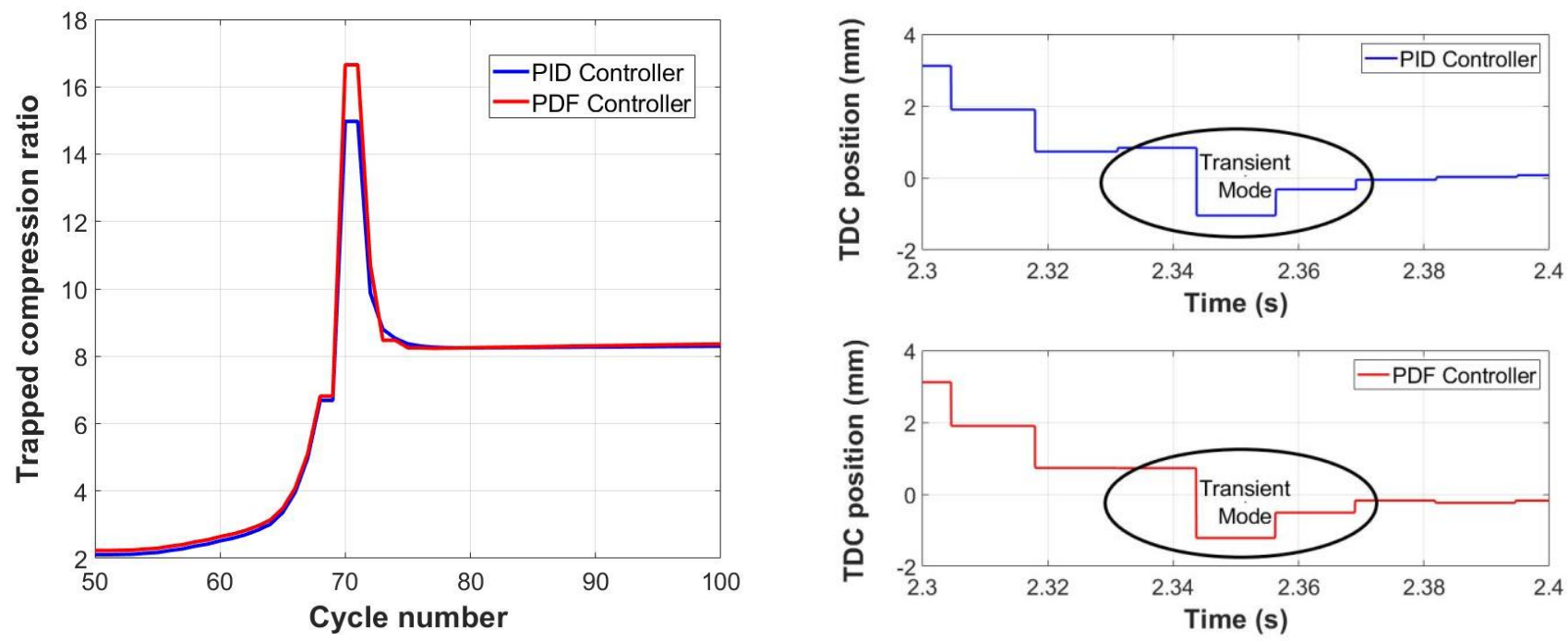

Figure 98: Deviations in trapped compression ratio and TDC position with PID and PDF controllers.
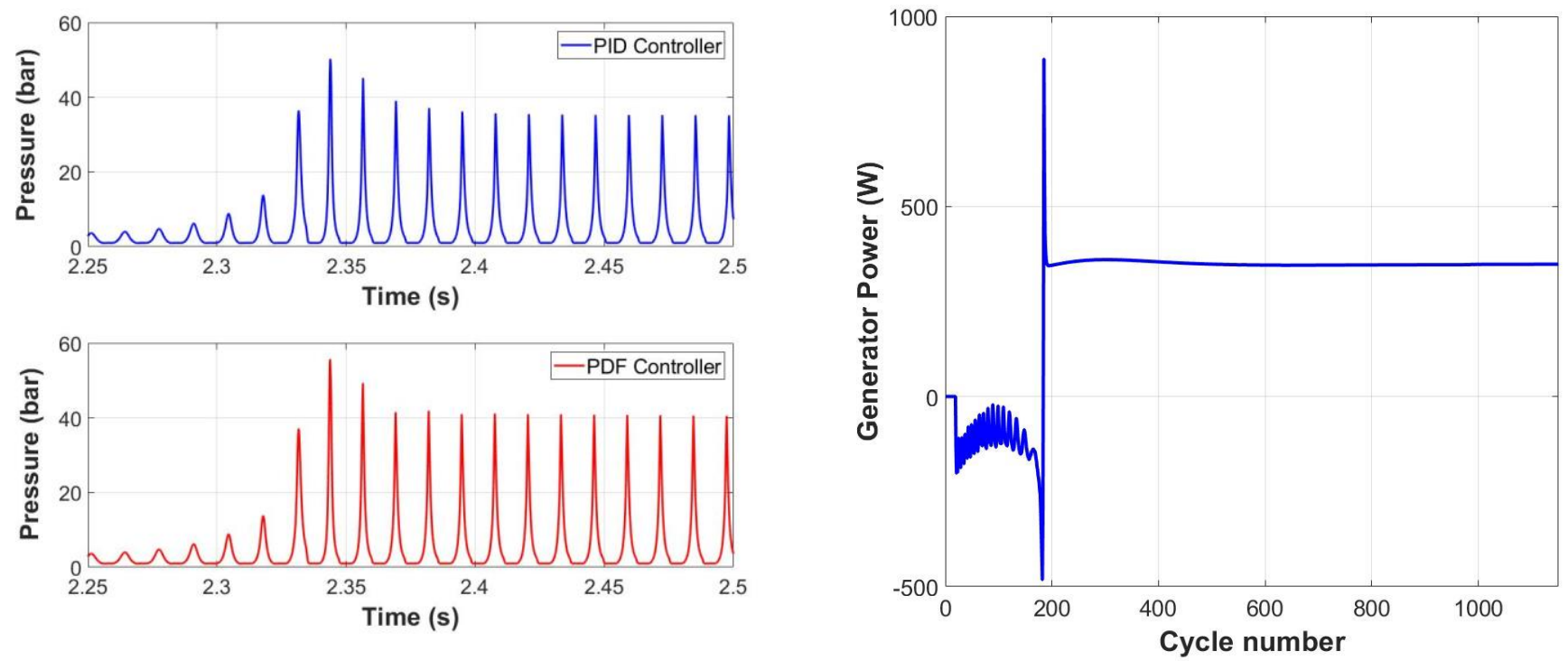

Figure 99: Deviations in cylinder pressures and LEM power output. 
During the transient mode between 2.34 to $2.37 \mathrm{~s}$, the controller detected an increase in the compression ratio. Therefore, the controller acted at $2.35 \mathrm{~s}$. As a result, the pressures, and the compression ratio deviations were reduced on the following cycles. Moreover, from Figure 99, the LEM power was high when the compression ratio was high. Later, the LEM power was gradually reduced with a reduction in the actual compression ratio. During the steady state operation, the LEM power was maintained relatively constant at $350 \mathrm{~W}$. Figure 100 shows the PID controller action due to step changes in the compression ratio.

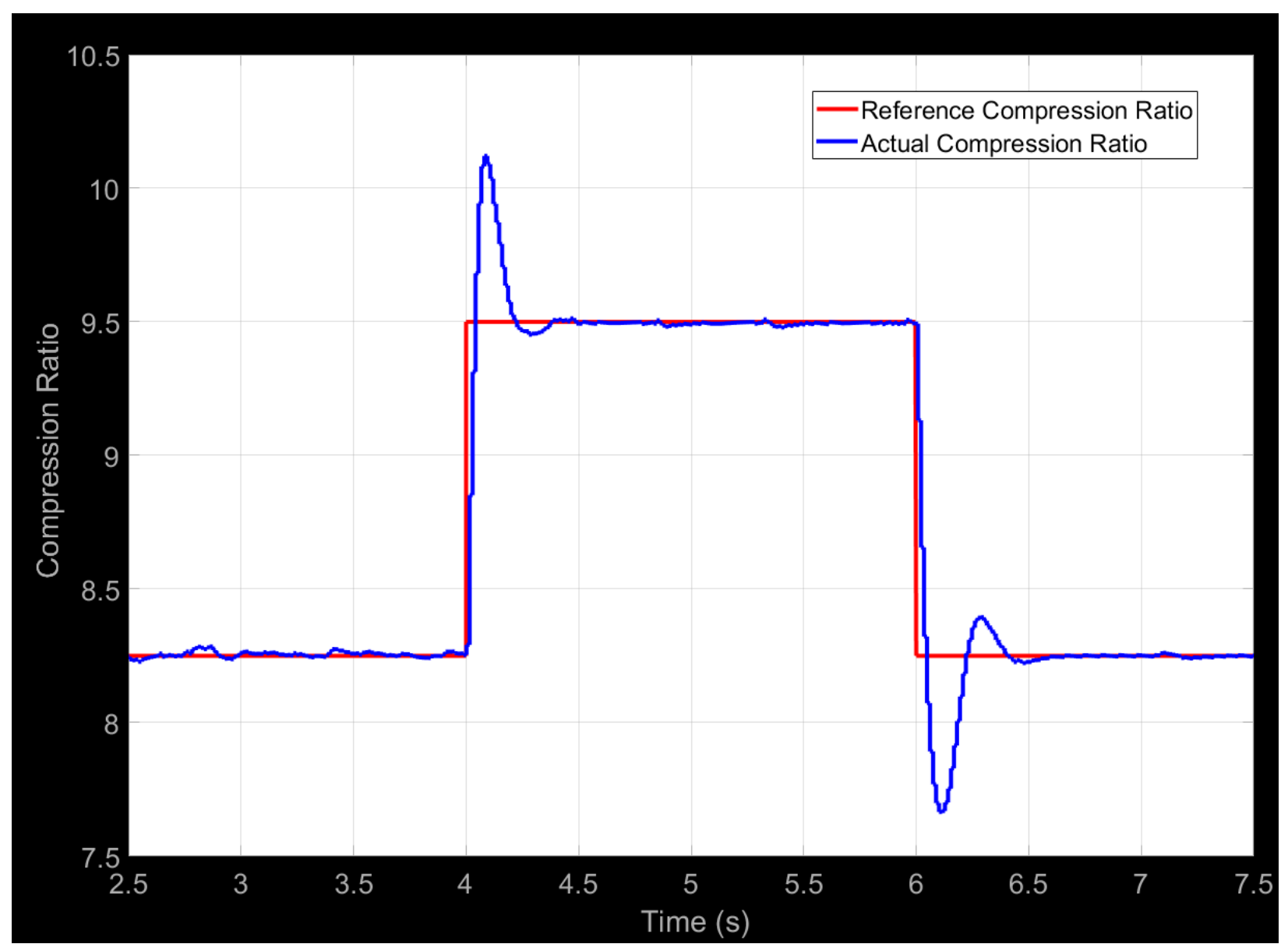

Figure 100: PID Controller action due to step changes in the compression ratio.

To test the feasibility of the energy management controller for transient operation, the compression ratio was increased from 8.3 to 9.5 at 4 seconds. Later, at 6 seconds, the 
compression ratio was again changed back to 8.3. The variations in the compression ratio were due to variations in the LEM load demand. Moreover, the control action was subjected to a constraint in this case. In order words, a saturation limit was applied to the LEM gain. The LEM gain was varied by only $+/-15 \%$ in any cycle. This saturation prevented the controller from taking immediate action. Moreover, this saturation limit was important especially in the FPEG system with rapid cycle-to-cycle variations. Without saturation limits, the external controller promoted cycle-to-cycle variations rather than reducing them. From Figure 100, the controller took corrective actions within $0.38 \mathrm{~s}$ for a step increase and a step decrease in the compression ratio. Based on the results, the controller was feasible to be used in the experimental FPEG system.

\subsubsection{Controller simulation - Dead center setpoint input}

The TDC and BDC clearance setpoint tracking was the most common method to control the FPEG piston motion [12, 16]. However, most of these control approaches were directed towards controlling the fuel load rather than the LEM load. In this dissertation, the focus was upon controlling the LEM load rather than the fuel input. Initially, the dead center clearance setpoints were provided as inputs to the numerical model. In this case, the effective stroke length was constant. This was because of controlling both the TDC and BDC locations. In other words, this configuration allowed the FPEG system to operate within the prescribed dead centers. Moreover, with fixed dead centers, the port locations were fixed at a particular location. As a result, this configuration allowed FPEG to operate similarly to the CSE system within the narrow frequency band. In addition, the ICE optimization was easy with this controller when compared to the compression ratio controller. This was due to fixed port geometry and stroke length for a given operation frequency. The controller responses with respect to dead center inputs are explained by a series of figures.

Figure 101 shows the deviation in the TDC and BDC tracking setpoint deviation and trapped compression ratio for both the controllers. From Figure 101, both the controllers had a similar response to the deviations. However, the slight deviations were observed. These deviations in the controller action were because of the differences in the action of multiple terms. In a PID controller, the action of the controller depended upon the deviation in the setpoints. The integral, differential and proportional terms controlled the LEM load based on the error calculated. However, in a PDF controller, the action was taken only by the integral term. The differential and 
proportional actions depended upon the actual process control variable rather than the error. These differences caused the differences in the trends of these controllers in Figure 101.
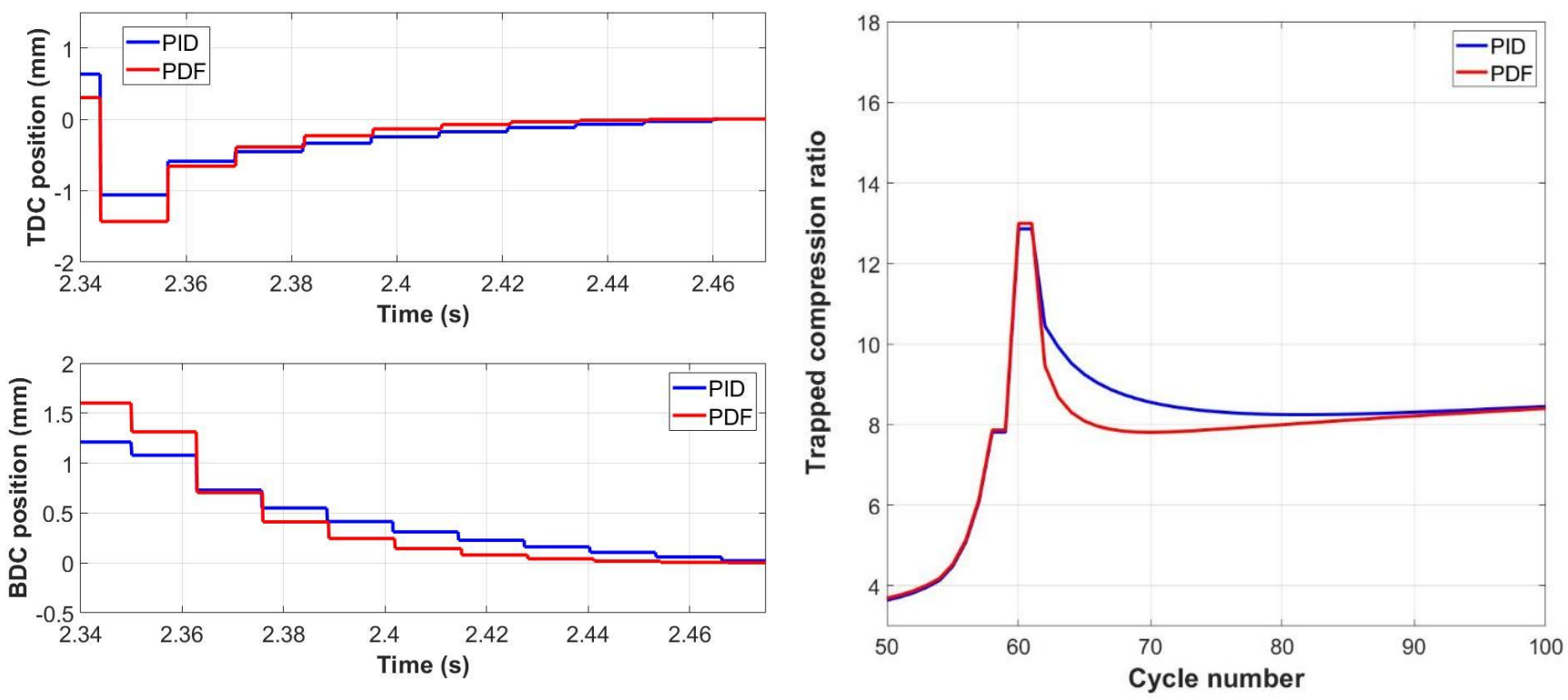

Figure 101: Deviations in dead center positions \& compression ratio with PID and PDF controllers for dead center setpoint inputs.

Similar to the compression ratio peak in Figure 98, the compression ratio also attained a high initial peak in this case. The PID and PDF controllers were unable to prevent the high initial peak. The main reason for that high peak was because of the delay in the error detection and the controller action. However, once the controller detected the error, the action was much rapid. This was clear from the reduction in the compression ratio from 13 to 8 within five consecutive cycles. From Figure 101, it is seen that the PDF controller had a significantly faster response than the PID controller. This was because of the better load handling capabilities of the PDF integral term. While this was one explanation, the manually tuned coefficients were also responsible for the deviation.

The cycle-to-cycle variations in the in-cylinder pressures for both the controllers are presented in Figure 102. Similar to cylinder pressures in Figure 99, the cylinder pressures were within reasonable limits. In the compression ratio control case, the pressures were increased to 60 bar. This was because of the high initial peak. Later, they were reduced to 30 bar within 5 cycles because of the controller action. In case of a dead center setpoint control, the cylinder pressures 
were increased to 40 bar initially. This was again because of the high initial peak. Later, it was reduced to 30 bar within 5 cycles because of controllers taking action on the deviations. Similar to the compression ratio control, both the PID and PDF controllers controlled the FPEG system during the transient mode. Therefore, this controller with dead center clearance setpoint inputs can also be used in the experimental system.
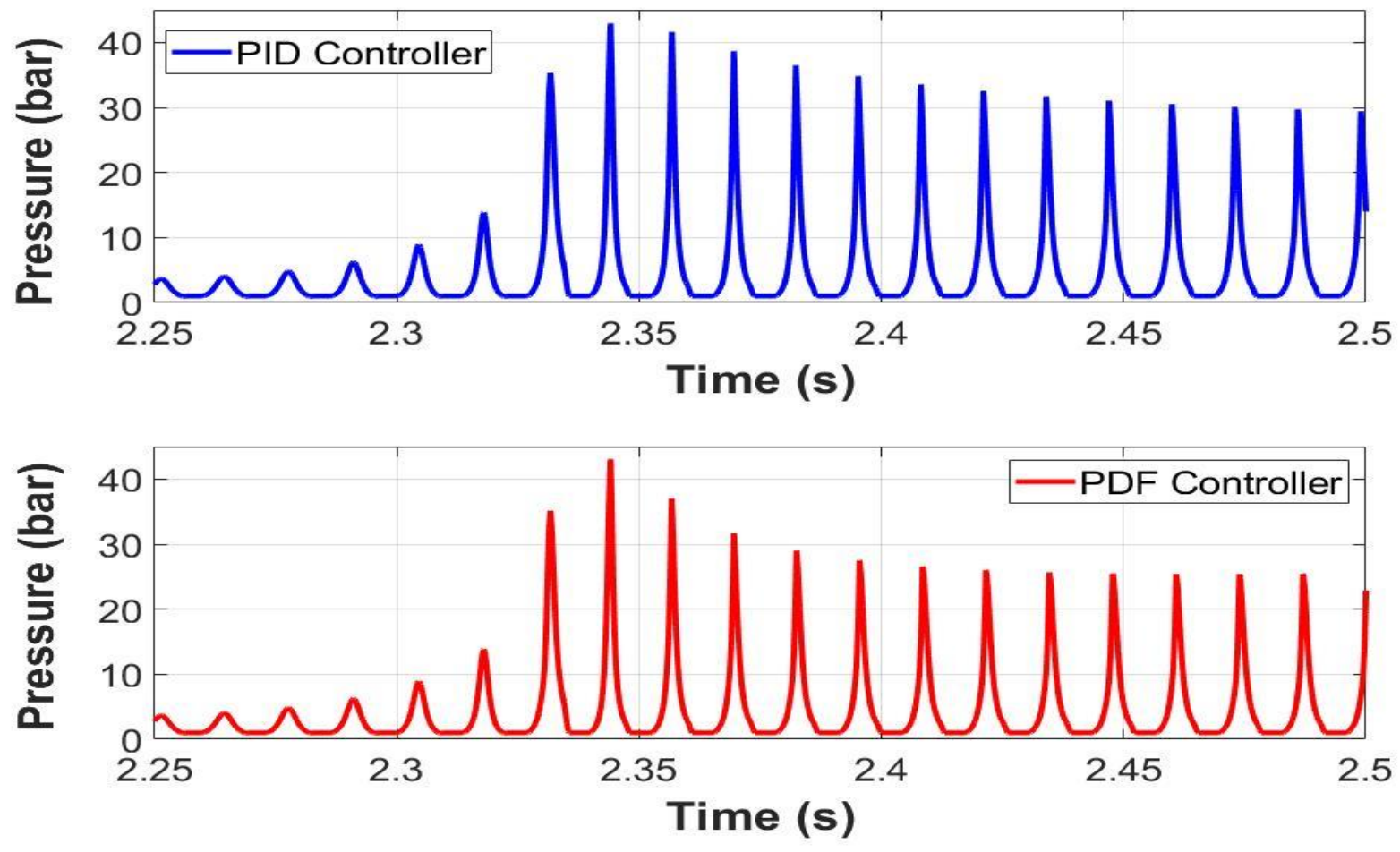

Figure 102: Cycle-to-cycle cylinder pressure variation with dead center clearance set point control.

\subsubsection{Controller simulation - Peak cylinder pressure input}

Many researchers have investigated FPEG control systems using compression ratio and dead center clearance setpoints. However, the FPEG control using peak cylinder pressure and midstroke velocity has not been explored until now. The upcoming sections build upon the feasibility of these control approaches. In this section, the peak cylinder pressure was provided as an input to both the controllers. The target value of the peak cylinder pressure was set at 35 bar for both the controllers. The results presented in this and the upcoming sections were fundamental with optimized controller coefficients. Additional control investigations were required to use these 
inputs as control variables in the FPEG system. Figure 103 shows the variations in the compression ratio and cylinder pressures with peak cylinder pressure as controller input.
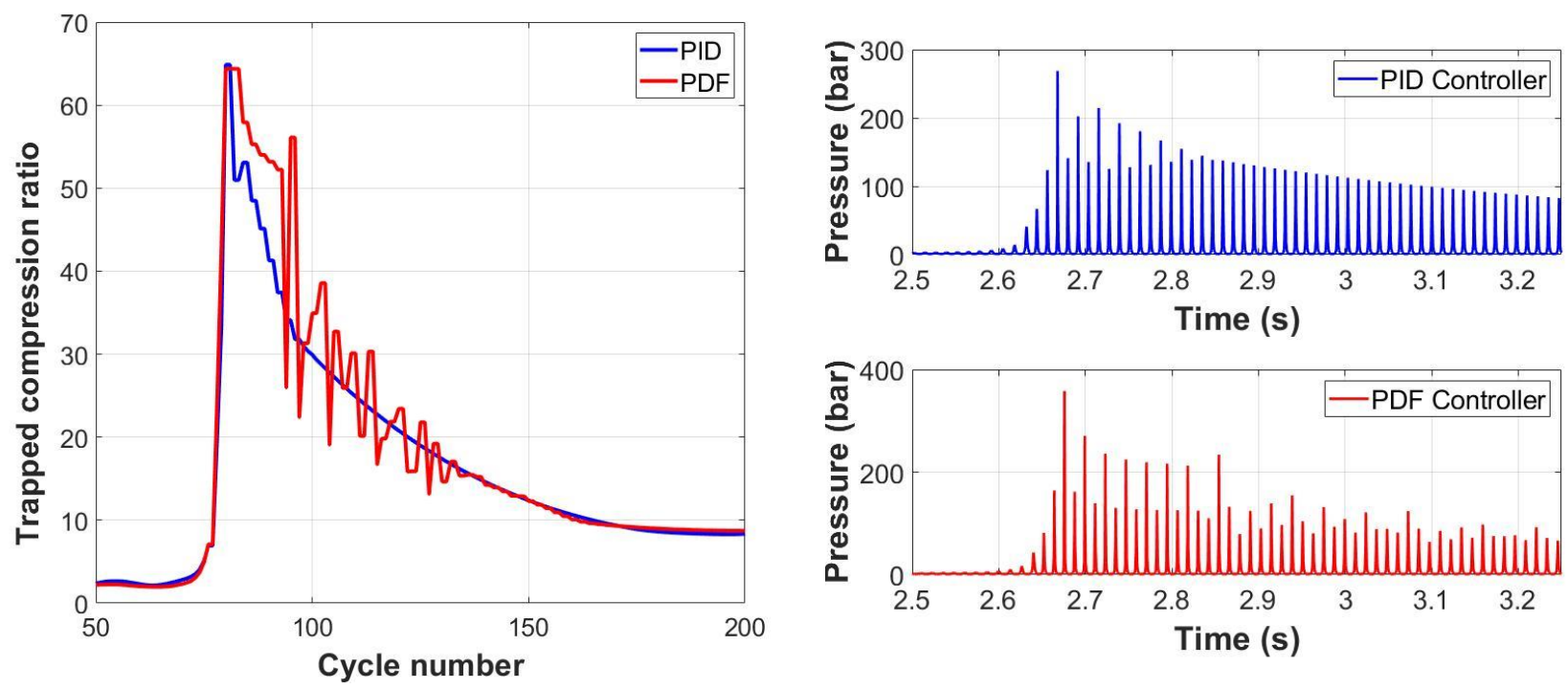

Figure 103: Cycle-to-cycle variations in compression ratio and cylinder pressures with peak cylinder pressure input.

The compression ratio, in this case, had a higher peak of 60 when compared to $15-20$ in the previous cases. The controllers had no control in avoiding the initial error peaks. This initial peak compression ratio of 60 has nothing to do with the controller selection. However, after detecting the peaks, the PID controller has smoother variations when compared to the PDF controller. One reason for such smoother variations was because of the good setpoint tracking ability of the PID controller. In a PID controller, the action was applied using proportional, integral, and differential terms on the error. This controller action on the error was responsible for the smoother variations in the PID controller. As mentioned earlier, the PDF was nonresponsive to the rapid load changes. This was because of the controller action defined by only the integral term in the PDF controller. The proportional and differential terms were eliminated in the forward path of the PDF controller. In other words, the proportional and differential actions were calculated from the actual pressure rather than the error in pressures. As a result, the PDF controller showed unsmooth variations during the transient process.

In addition to this, a slight increase or decrease in the compression ratio deviations caused rapid cylinder pressure variations. This was evident from the pressure variations in Figure 103. From 
Figure 103, because of the high compression ratio, the cylinder pressure increased to 250 bar. However, in previous cases, the pressures were around 60 bar. Moreover, the modeled cylinder pressures were influenced by the multiple submodels within the system. As a result, the PID and PDF controllers took corrective action within 100 cycles to eliminate the error. These variations were more clearly seen in the TDC position deviations shown in Figure 104.
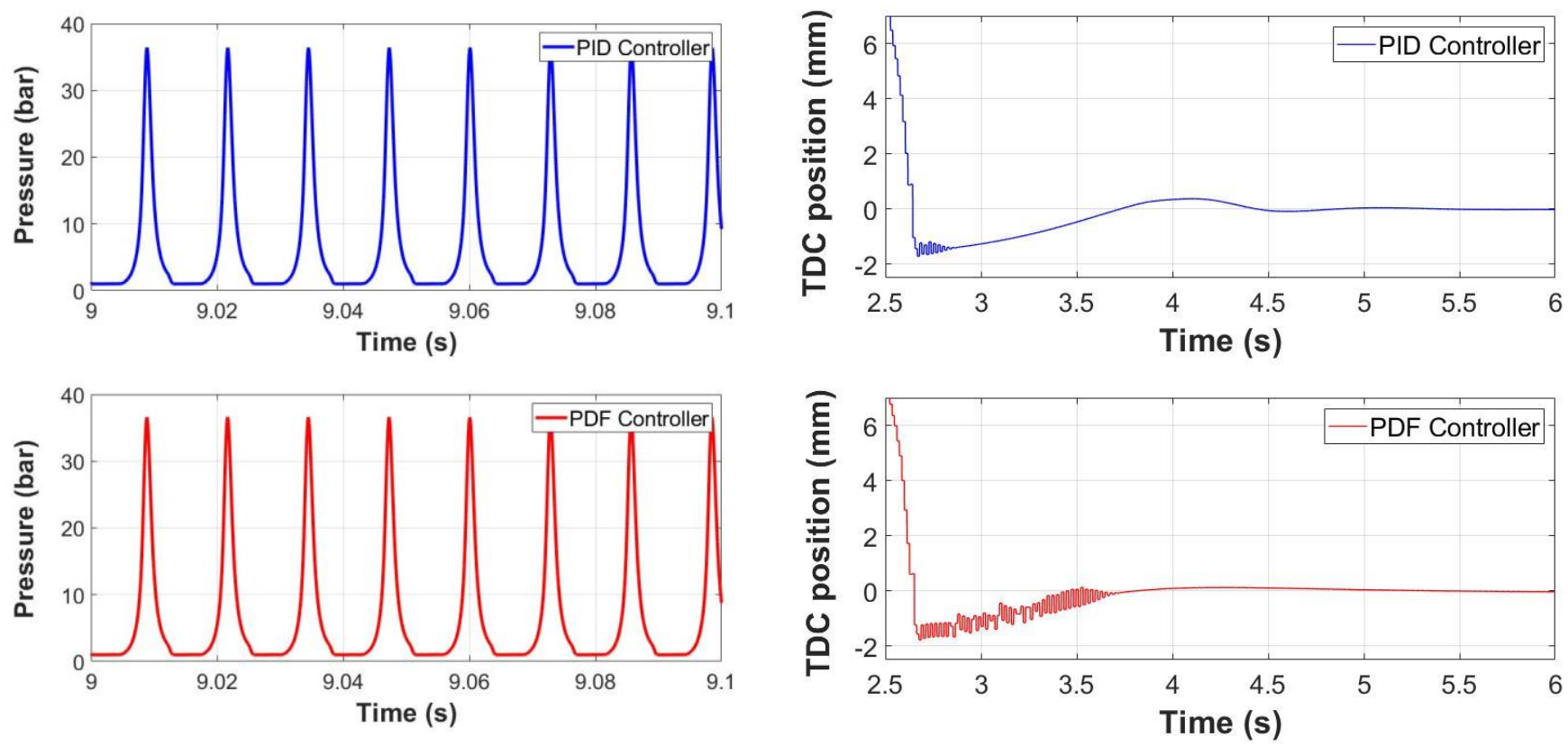

Figure 104: Steady state in-cylinder pressure \& dead center positions with maximum pressure as an input to the controllers.

From Figure 104, it is evident that the controller took $1 \mathrm{~s}$ to $1.5 \mathrm{~s}$ to obtain a stable value for compression ratio and dead center clearance setpoints. In terms of frequency, these values correspond to 70-100 cycles. The controllers in the previous cases controlled the variations in 510 cycles. The delayed controller action with peak pressure input makes it inferior when compared to other inputs. For example, in previous cases, the controller regulated the dynamics initially. Later, multiple submodels that were dependent upon dynamics were controlled. However, in case of a peak pressure input, multiple submodels were controlled initially. Later, in the end, the dynamics were controlled. As a result, this controller was inferior when compared to the controllers in previous sections. 
Though the compression ratio was high initially, the controllers took the corrective action on peak cylinder pressures. The only difference was the gradual action rather than the swift action in previous cases. However, the system reached a steady state. This was evident from a steady peak cylinder pressure of 35 bar as seen in Figure 104. Based on the results, it is evident to use peak pressure as an input to the controllers for steady FPEG operation. However, a detailed investigation was required to achieve rapid corrective action.

\subsubsection{Controller simulation - Mid-stroke velocity input}

With the stiff spring addition, the dynamics were almost sinusoidal and symmetric around the neutral position. The dynamics in such a spring-dominant system were defined mainly by the stiff springs. In the experimental FPEG system, the peak spring and peak cylinder forces were of the magnitude of $11 \mathrm{kN}$ and $5 \mathrm{kN}$. The peak spring force magnitude was more than twice the peak cylinder force magnitudes. In this section, the mid-stroke piston velocity was provided as an input to the controllers. In the previous cases, TDC was defined and provided to the controller. In this case, the neutral position and target mid-stroke velocities were provided to the controller. The target mid-stroke velocity was set to $7 \mathrm{~m} / \mathrm{s}$. This value was calculated based on the mean piston speed of the experimental FPEG system operating at a frequency of $120 \mathrm{~Hz}$ and $30 \mathrm{~mm}$ effective stroke. Figure 105 shows the mid-stroke velocity variations for both PID \& PDF controller.

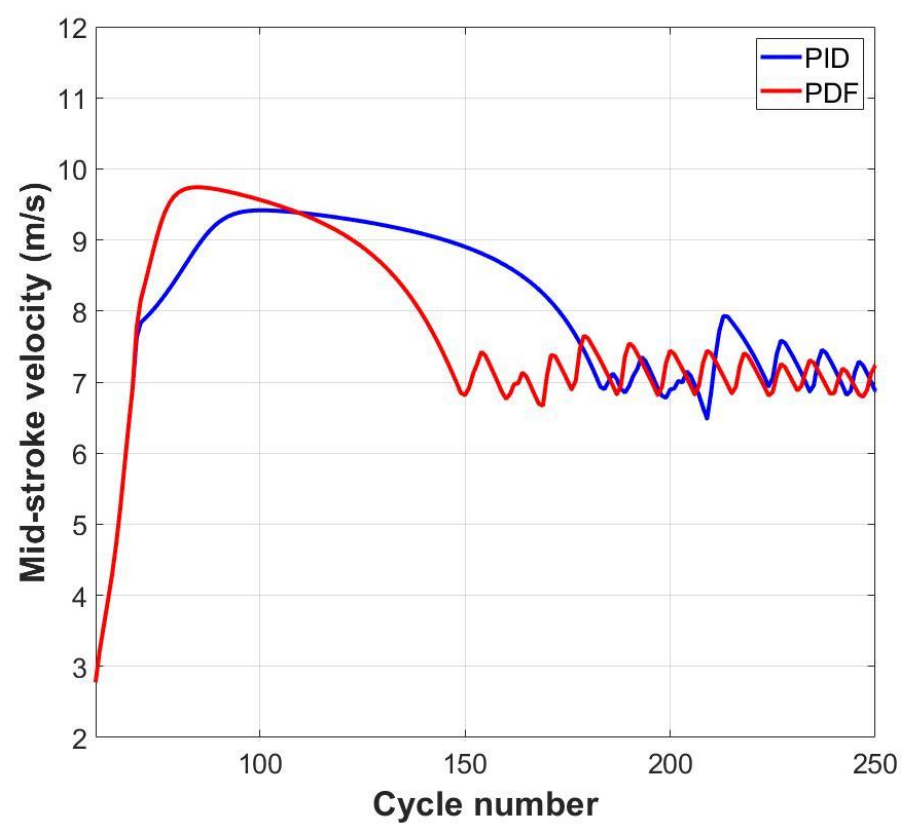

Figure 105: Variations in mid-stroke velocity with mid-stroke velocity control input. 
Based on Figure 105, the mid-stroke velocities rose to the value of $10 \mathrm{~m} / \mathrm{s}$. This was because of the high initial peak. Later, they were gradually reduced to the setpoint value of $7 \mathrm{~m} / \mathrm{s}$. However, unlike previous cases, the controllers failed to maintain the mid-stroke velocity of $7 \mathrm{~m} / \mathrm{s}$. It was evident from cycle-to-cycle variations in the mid-stroke velocities from 200 to 250 cycles in Figure 105. The cycle-to-cycle variations were because of the lack of proper information on dead center locations to the controller. In other words, the controllers were underdefined. In the previous three cases, the dead center positions were defined either by using the compression ratio, dead center clearance setpoints, and maximum cylinder pressures. However, in this case, only mid-stroke velocity was provided without any information about dead center locations. As a result, the FPEG system found its own dead center locations. These locations were defined based on the nonlinear nature of the forces acting on the piston. These dead center locations were not ideal. Moreover, they were deviated from the actual dead center setpoints. The deviations are shown in Figure 106.

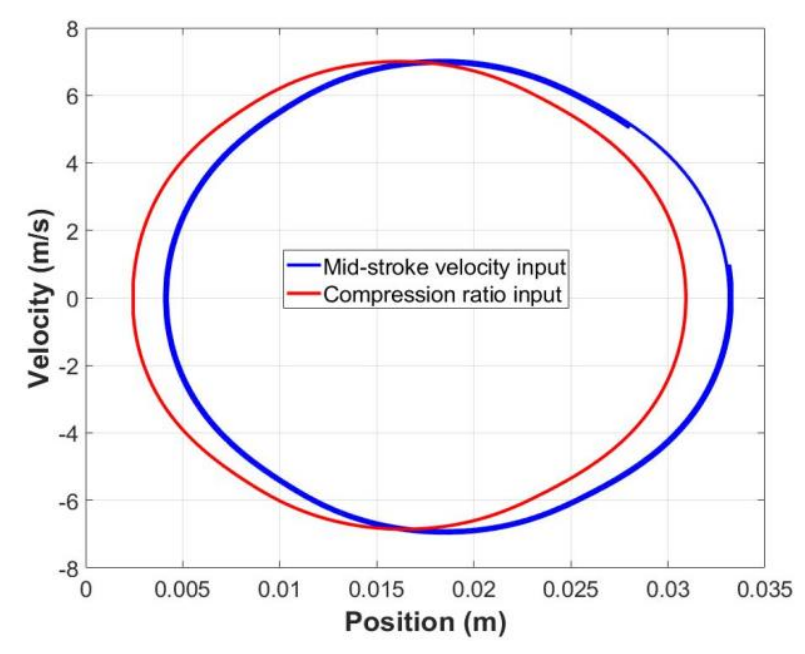

Figure 106: Velocity versus position distribution during steady state with compression ratio and mid-stroke velocity input to the controllers.

Based on Figure 106, the dead center positions were shifted towards the right when compared to the compression ratio input. One reason was due to the neutral position of the system. In all the control inputs, the neutral position was set at $18 \mathrm{~mm}$ from TDC. However, since the dead center positions were defined for compression ratio input, this neutral point was modified. In this case, however, this neutral position was not modified. As a result, the system found its own dead center based on the non-linear nature of forces. Owing to this dead center deviations, the port locations, 
operations, and combustion attributes were also affected. From Figure 106, the mid-stroke velocity was the same for both the mid-stroke velocity and trapped compression ratio controllers. However, the dynamics were shifted towards the right in a mid-stroke velocity approach. The compression ratio and cylinder pressures were also reduced in this case. The compression ratio variations during the transient and steady state operations are shown in Figure 107. Based on Figure 107, both the PID \& PDF controllers behaved in the same way.
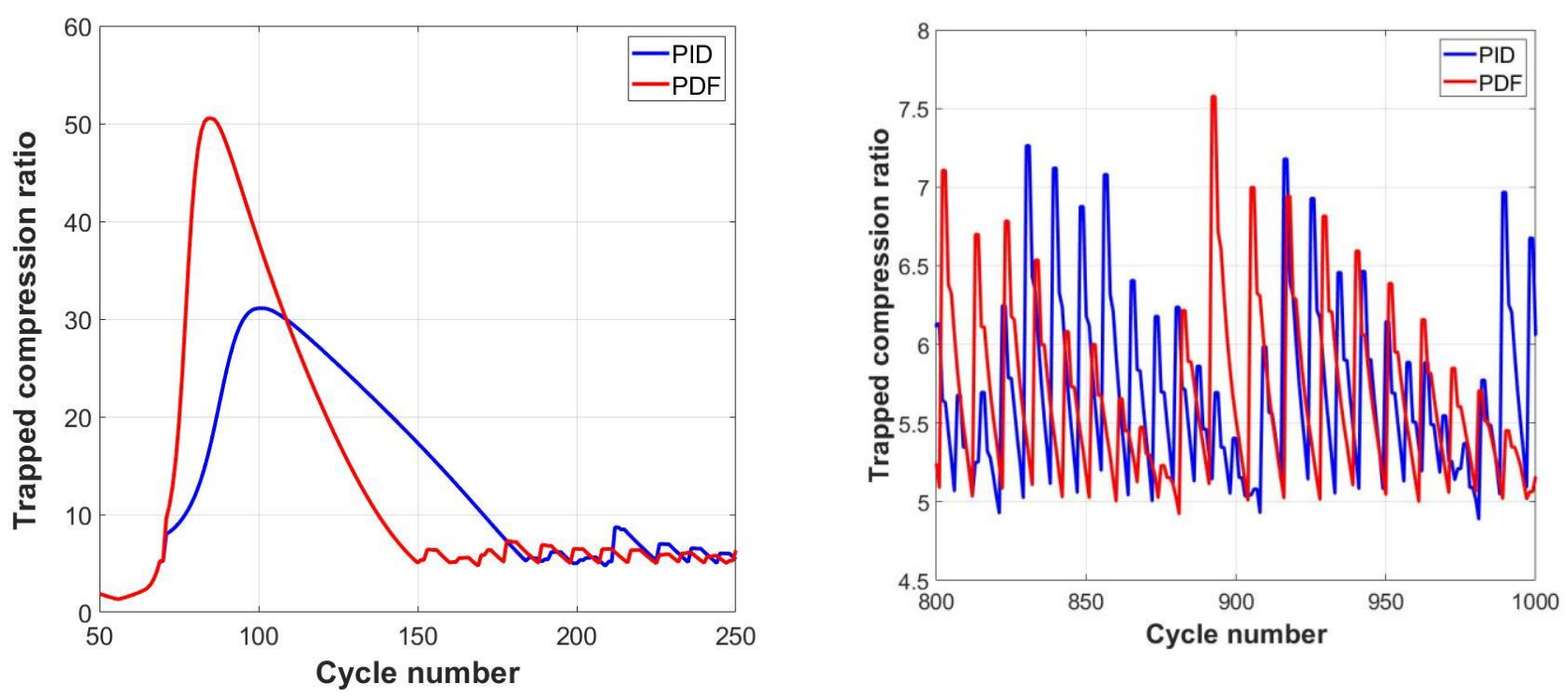

Figure 107: Variation in the trapped compression ratio during transience and steady state operation.

From Figure 106 and 107, the deviation in the FPEG system dynamics changed the FPEG operational characteristics. From Figure 107, the compression ratio was found to vary between 5-7.5 during the steady state operation. Due to a reduction in the compression ratio, the cylinder pressures were also reduced to 10-15 bar. Further, these pressures reduced the indicated and electrical performance. In addition to the compression ratio, the expansion ratio was also reduced. This was because of the EPO location moving closer to TDC. Due to this, the EGR percentage was also increased. Based on the results, the mid-stroke velocity itself was not enough to control the FPEG system. The mid-stroke velocity needs to be used in conjunction with peak pressure, compression ratio, and dead center set point control variables. These additional control variables provided the information on dead centers to the controller. Due to the additional variables, the mid-stroke velocity controller is now properly defined. With the updated mid-stroke velocity controller, the FPEG can be operated steadily at an optimized performance. 


\subsubsection{Effect of model time step on controller behavior}

In PID and PDF controllers, the integral and differential term actions were time-dependent. In other words, they were discrete time-based integration and differentiation terms. The sample time at which these terms were calculated will change the output. An investigation was conducted to understand the effect of the model time step on the controller behavior. In this case, the peak cylinder pressure was provided as an input to the controller. Four different time steps were considered in this investigation. They were $0.5 \mu \mathrm{s}, 5 \mu \mathrm{s}, 7.5 \mu \mathrm{s}$, and $10 \mu \mathrm{s}$. The changes in the compression ratio and dynamics with different time steps were shown in Figure 108.
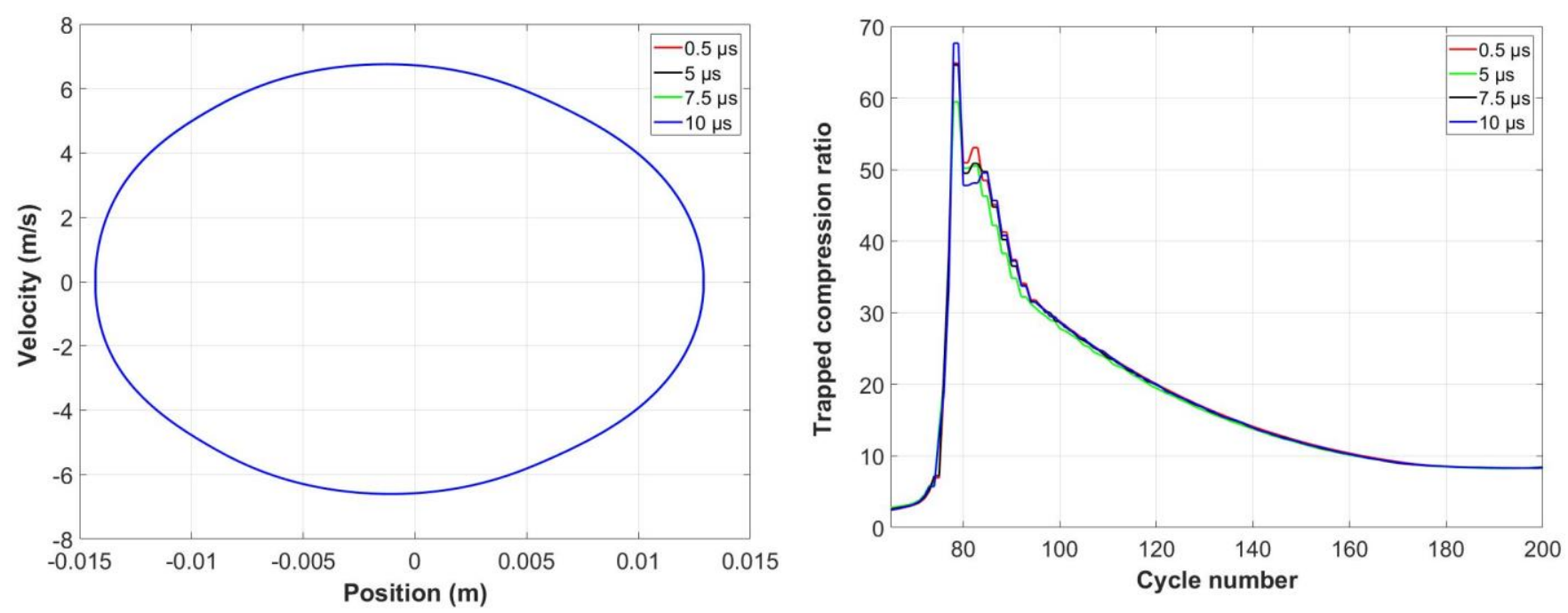

Figure 108: Variation in the trapped compression ratio and dynamics with different time steps during the transient mode operation.

From Figure 108, the numerical model time step had no effect on the controller behavior. The controllers with different time steps reached the steady state within the same time. The only difference was the difference in the control actions taken at different time steps. These differences were evident from the oscillations in the trapped compression ratio from 80 to 100 cycles. Moreover, the system attained the same dynamics for all the time steps during the steady state 
operation. Based on these, only the controller coefficients had a major impact on the controller setpoint ability.

\subsubsection{Cycle-to-cycle variations}

The addition of stiff mechanical springs reduces the cycle-to-cycle variations in the compression ratio and cylinder pressures. To test the proposed hypothesis, a stochastic distribution was considered for the modeled combustion duration and efficiencies. The same hypothesis was applied by Robinson et al. for a dual cylinder FPEG system with springs [45]. In reality, these factors depend upon cylinder contents, port locations, and piston dynamics. The numerical model cannot capture these complex relationships. Hence, the stochastic components were added to the combustion efficiency and combustion duration to understand the cycle-to-cycle variations. The stochastic characteristics imposed on the combustion parameters are shown in Figure 109 [124]. The combustion efficiency was varied from $90 \%$ to $100 \%$ with a mean of $95 \%$. The

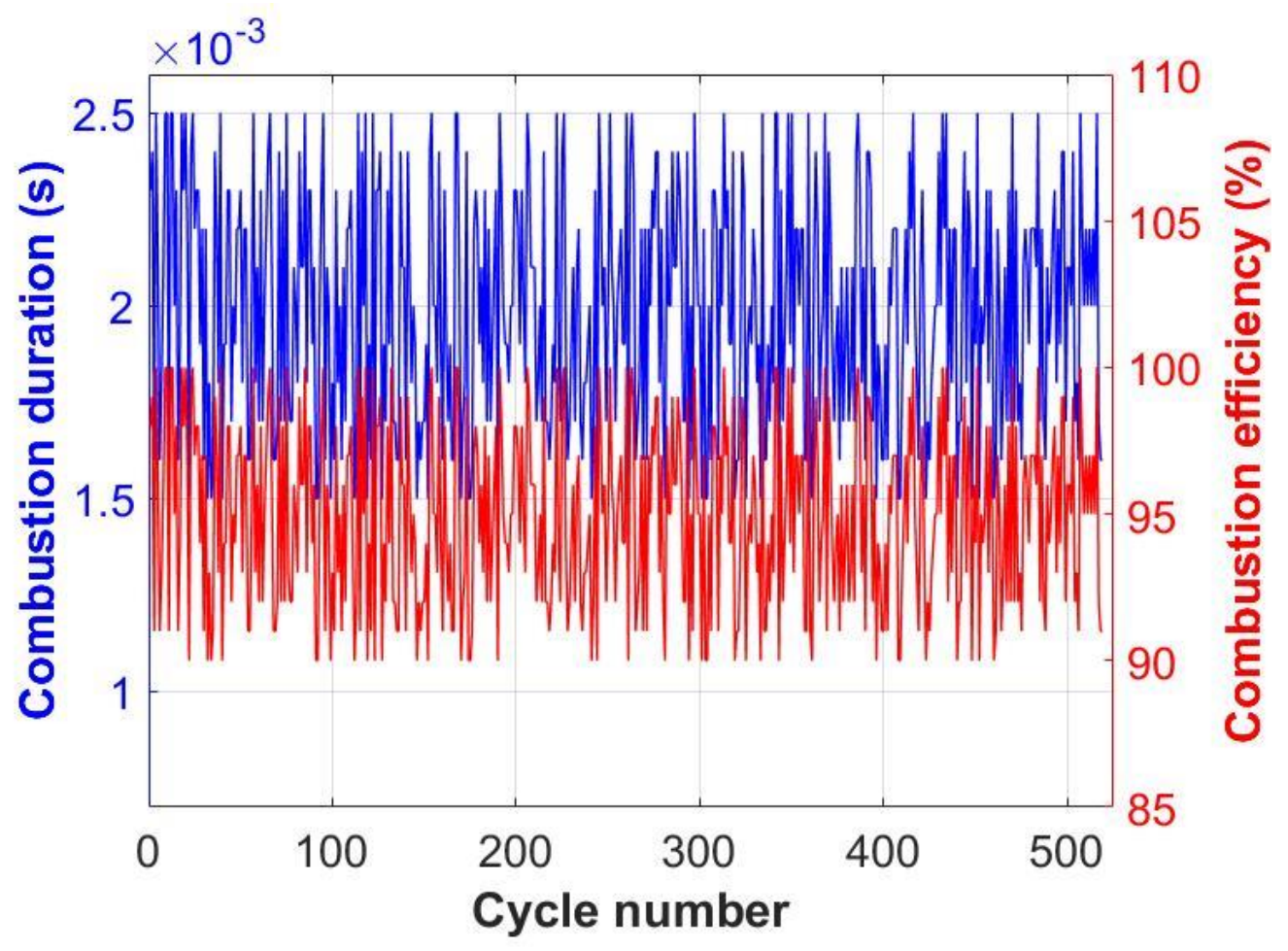

Figure 109: Stochastic variations imposed on combustion duration and combustion efficiency. 
combustion duration was changed from $1.5 \mathrm{~ms}$ to $2.5 \mathrm{~ms}$ with a mean of $2.0 \mathrm{~ms}$. Apart from these variables, all other parameters were held constant as given in Table 2.
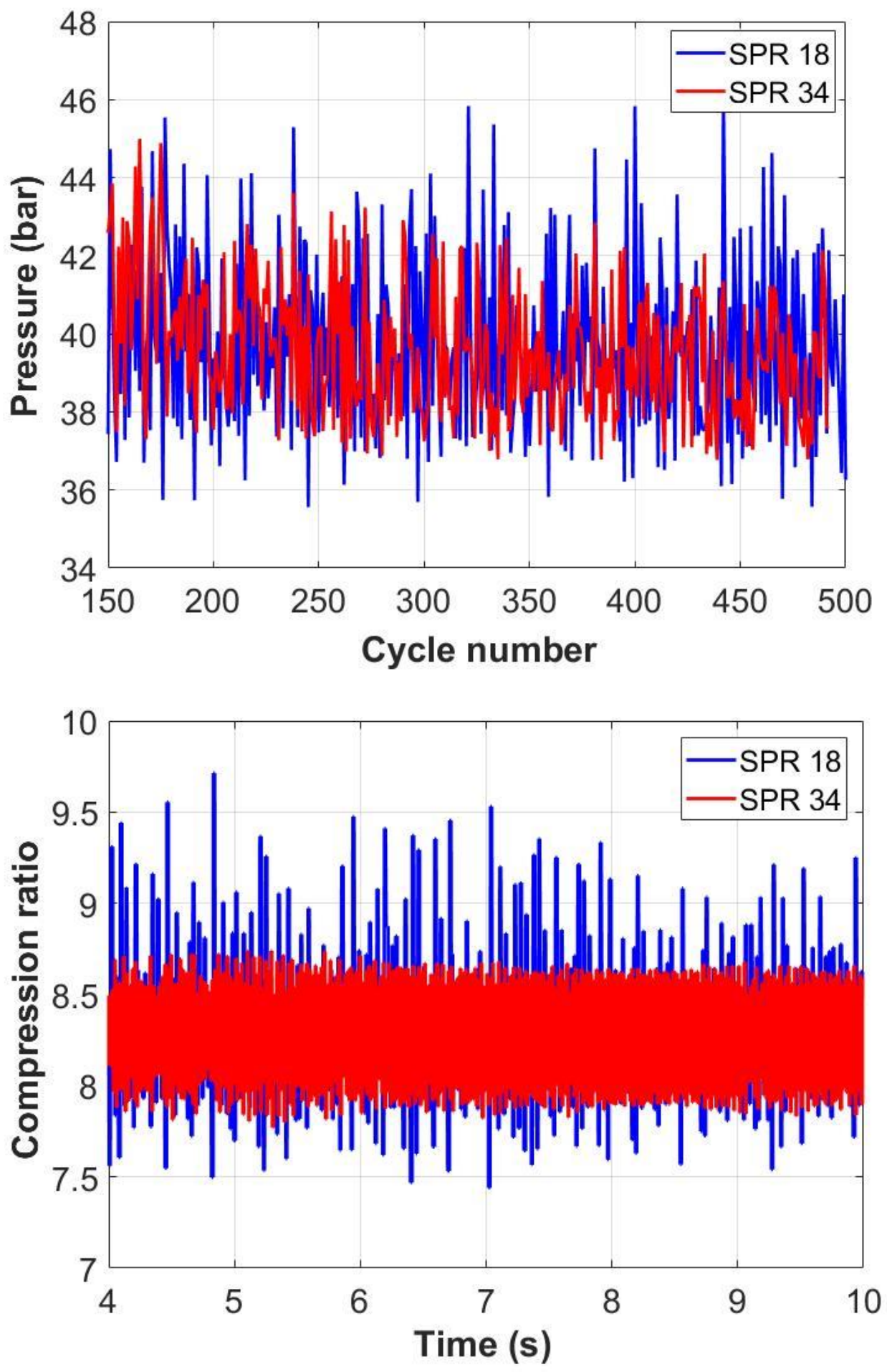

Figure 110: Variations in cylinder pressure and compression ratio with increasing spring stiffness. 
Figure 110 shows the variations in peak cylinder pressures and compression ratio for the lowest and the highest spring number of 18 and 34 . The spring number of 18 corresponds to the spring stiffness of $495 \mathrm{kN} / \mathrm{m}$. The spring number of 34 corresponds to the spring stiffness of $935 \mathrm{kN} / \mathrm{m}$. From Figure 110, the cycle-to-cycle variations were lower for 34 springs when compared to the 18 spring FPEG system. The same trend was observed in the compression ratio variations.

The cycle-to-cycle variations presented in Figure 110 were evaluated by using a coefficient of variation (COV). The COV's were calculated by using the standard deviation divided by the mean of that variable. Figure 111 shows the COV of the compression ratio and peak pressures with increasing spring stiffness.

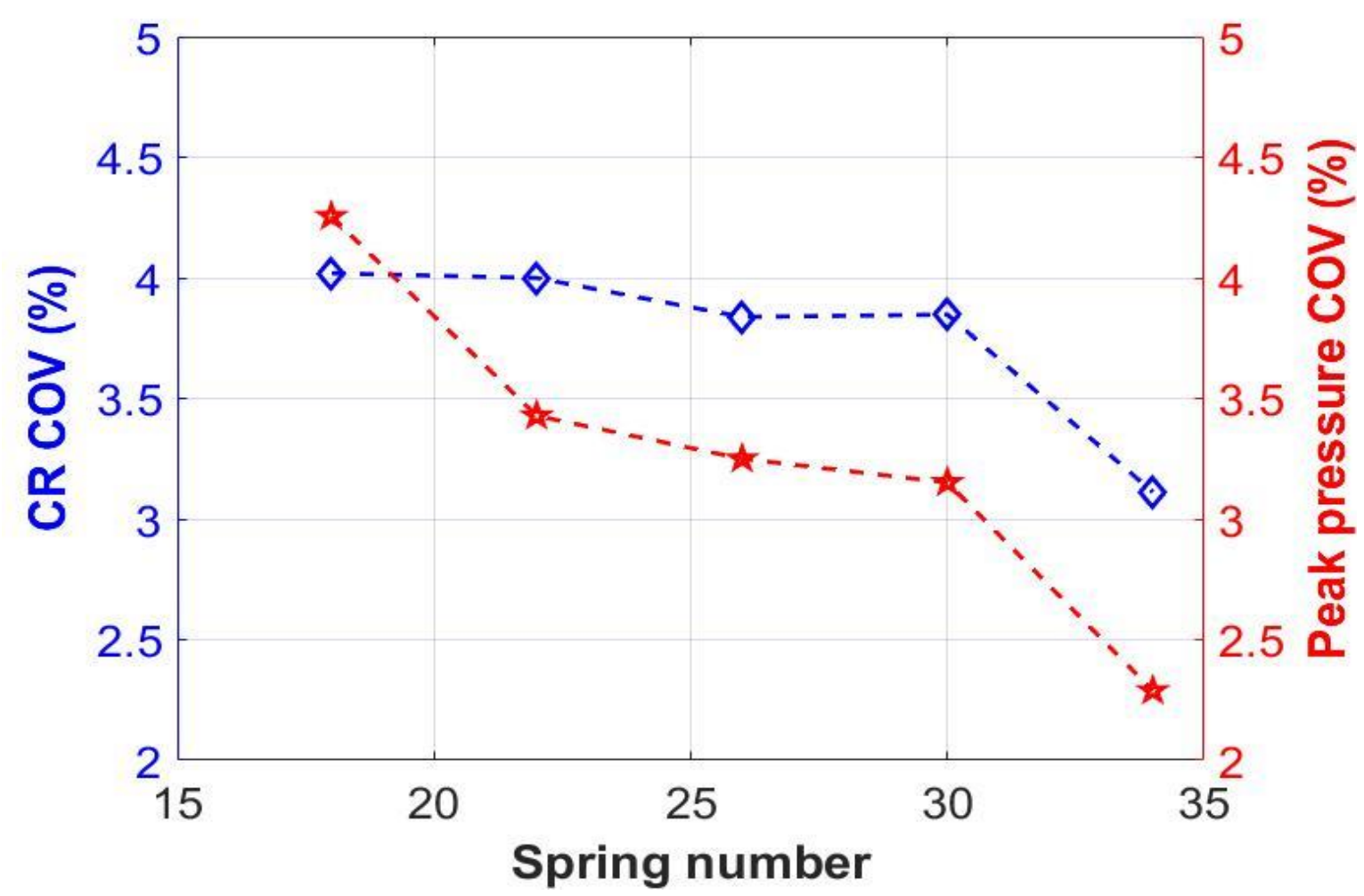

Figure 111: COV of compression ratio and peak pressures with increasing spring stiffness.

As seen in Figure 111, the COV of the compression ratio was reduced from $4.1 \%$ to $3.1 \%$. The COV of peak pressures was reduced from $4.3 \%$ to $2.3 \%$. Without the stochastic nature, the COV was found to be less than $1 \%$ with controllers in action. This was due to the controllers governing the outputs of multiple submodels. Moreover, without stochastics, the operating conditions 
remained the same. For an experimental FPEG system with a spring stiffness of $575 \mathrm{kN} / \mathrm{m}$, the COV in peak pressures was found to be $5 \%$. This value was slightly higher than the modelpredicted COV of peak pressures. The model-predicted COV was found to be $3.5 \%$ for the same spring stiffness. From Figure 111, it is evident that the addition of stiff springs in the FPEG system reduced the cycle-to-cycle variations. In addition to this, the cycle-to-cycle variations were also investigated with different control algorithms. In this case, the same number of springs were considered. Similar to the previous case, the cycle-to-cycle variations were evaluated using the COV. Figure 112 shows the cycle-to-cycle variations in the compression ratio and peak pressures with different control inputs [125]

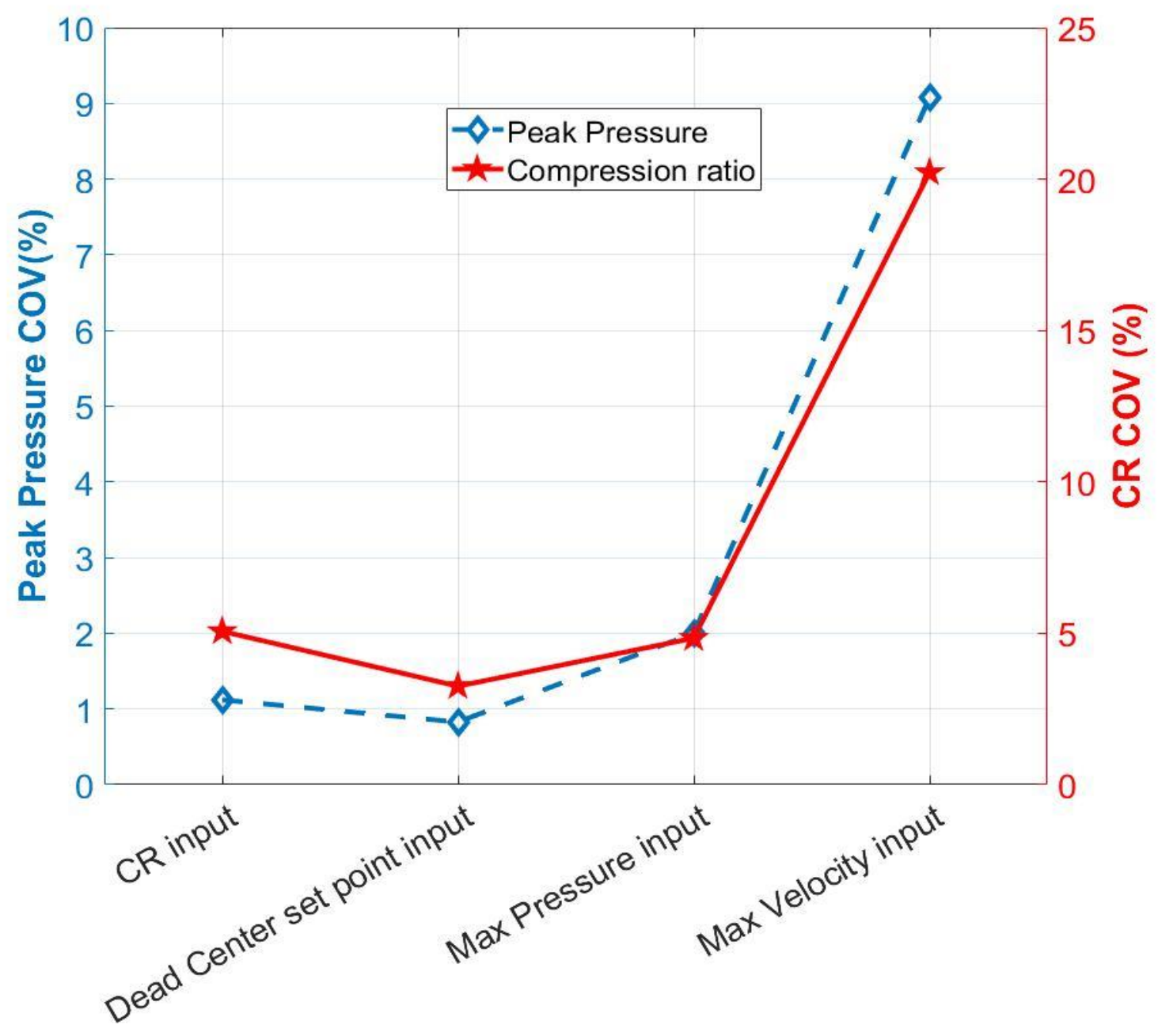

Figure 112: Cycle-to-cycle variations in compression ratio and peak cylinder pressures with different controller inputs. 
Figure 112, it is evident that the COV was maintained below $5 \%$ for all the controllers except the mid-stroke velocity controller. The COV of peak pressure and compression ratio rose to $8 \%$ and $20 \%$ for the mid-stroke velocity input. Therefore, based on the modeling results, the FPEG system can be controlled with all the controllers except the mid-stroke velocity. As mentioned earlier, the mid-stroke velocity controller should be used in conjunction with other controllers. In other words, the mid-stroke velocity controller was underdefined. Though preliminary analysis was conducted in this dissertation, a more detailed analysis was required.

\subsection{MBT timing \& ignition signal controller}

MBT timing in an FPEG system referred to the optimized ignition timing for the maximized engine thrust. In CSE systems, the MBT timing was obtained by carrying out the parametric sweep studies. These studies require a substantial experimental effort. To avoid this, an MBT timing detection algorithm was devised for an FPEG system. The algorithm was based on the closedloop control method developed by Zhu et al. [126]. The devised algorithm used time-based cylinder pressure as an input. Later, the MBT timing was obtained by locating the zero acceleration or maximum velocity of the net pressure. This MBT detection process was valid only for the SI engines with only one peak HRR process. Moreover, a closed-loop controller was developed at the modeling level to obtain the MBT timing. Usually, the combustion process in an SI FPEG engine was defined using a time-based MFB profile. This MFB curve defines the combustion energy release at each time step after the ignition signal. A typical MFB signal along with its first and second order derivatives is shown in Figure 113.

The first derivative of the MFB curve represented the combustion process velocity. In order words, the combustion process velocity was proportional to the HRR. The second derivative of MFB represented the acceleration of the combustion process by volume. From Figure 113, the 50\% of MFB coincided with the location of peak HRR. The peak HRR location was the same as the zero acceleration of the MFB curve. This was evident from the dotted brown line in Figure 113. Based on various algorithms and experimental tests, the $50 \%$ MFB occurs at around $8^{\circ}$ to $10^{\circ}$ after TDC in a CSE [126]. The degrees after or before TDC had no meaning in an FPEG system. Hence, the $50 \%$ MFB location was defined in terms of displacement after TDC. In this investigation, the $50 \%$ MFB location was assumed to occur at $0.24 \mathrm{~mm}$ after TDC. This $0.24 \mathrm{~mm}$ corresponds to $8^{\circ}$ to $10^{\circ}$ after TDC in CSE of the same size and frequency. This value was calculated based on the effective stroke length, instantaneous time, and operating frequency. Initially, this value was 
provided as an input to the numerical model. Moreover, this value was defined a variable so that it can be changed depending upon the requirements.

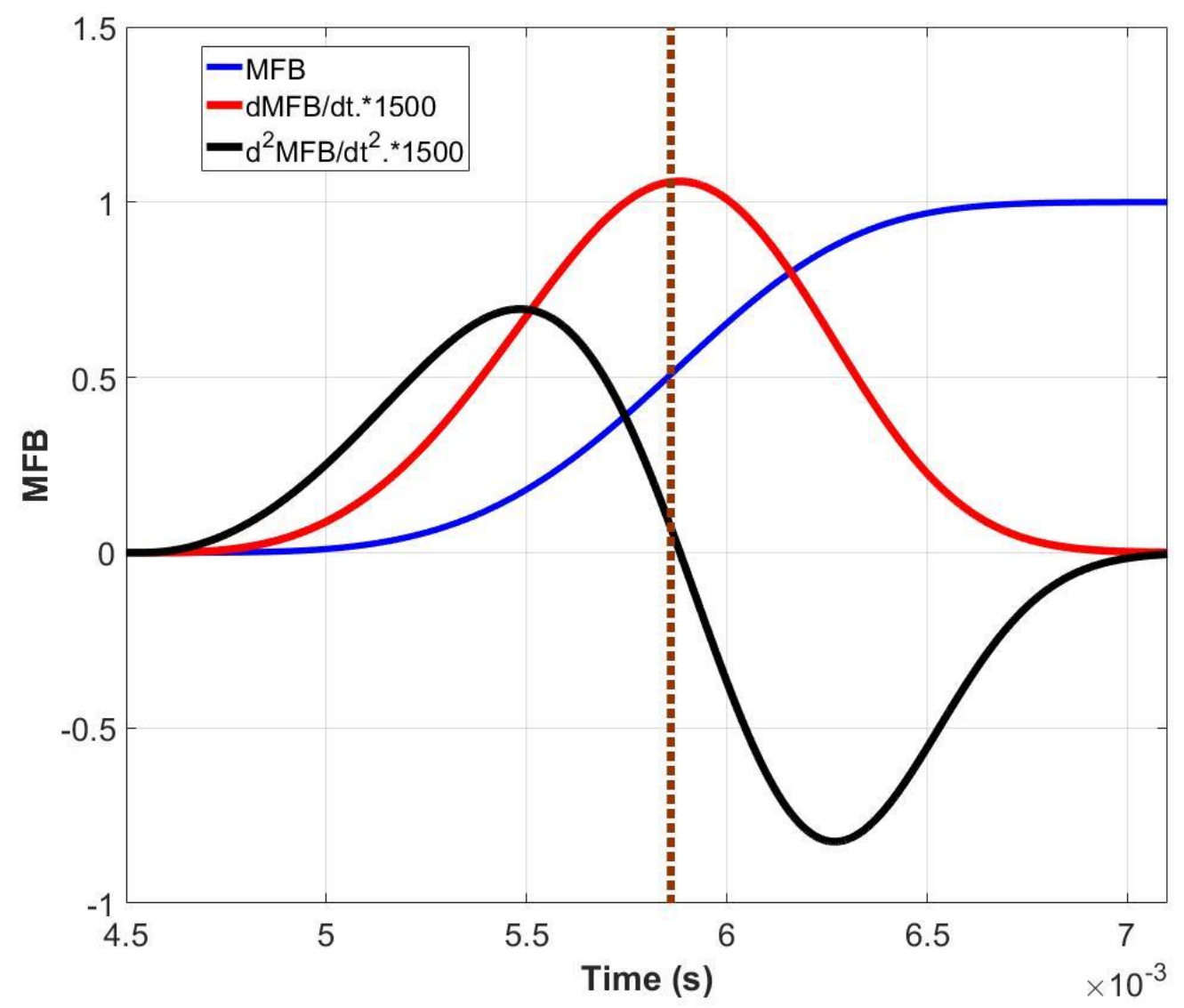

Figure 113: Distribution of MFB and its derivatives after igniting the mixture.

The MFB profile cannot be calculated during the FPEG operation. Therefore, the MFB profile was defined by using Rassweiler-Withrow method [126]. This method used cylinder pressure as an input. Moreover, it also used the cylinder volume at the ignition location as a reference. Later, the pressure increase after the ignition was calculated at each time step. In other words, the net pressure was calculated at each time step. This net pressure at each time step indicated the MFB percentage at that time step. Initially, the net pressure change between any time steps was calculated by the following equation in Eq. 3 .

$$
\Delta P_{i}=\left\{P_{i+1}-P_{i}\left[\frac{V_{i}}{V_{i+1}}\right]^{k}\right\} \frac{V_{i}}{V_{\text {spark }}}
$$




$$
\begin{gathered}
P_{n e t, i}=P_{n e t, i-1}+\Delta P_{i} \\
M F B_{i}=\frac{P_{n e t, i}}{P_{n e t, f}}
\end{gathered}
$$

In Eq. 3, the term ' $i$ ' represents the current time step. The term ' $P$ ' is the cylinder pressure. The terms ' $V$ ' and ' $V_{\text {spark }}$ ' represent the instantaneous volume and volume at the instant of ignition.

At first, the MFB profile was obtained based on net pressure. Later, the zero acceleration and $50 \%$ MFB locations were calculated. One can use either one of these methods for calculating the $50 \%$ MFB location. However, the use of these two approaches eliminated the time-step dependency of the numerical model. If the time step was high, the numerical model cannot capture the exact $50 \%$ MFB location. Hence, both the $50 \%$ MFB and the zero acceleration locations were used. The submodel for the $50 \%$ MFB location and MBT timing is shown in Figure 114. The submodel in Figure 114 was divided into three sections. The first set of blocks represented the differentiation and logical operations on the left. These blocks calculated the location of $50 \%$ MFB. The outputs of these blocks were binary. As seen in Figure 114, two approaches were used to calculate the 50\% MFB location. The first approach directly used the MFB signal calculated from the net pressure. The second approach located the zero acceleration location of the MFB signal.

The binary output of the $50 \%$ MFB location was used to enable a triggered subsystem. This triggered subsystem calculated the 50\% MFB location in terms of displacement after TDC. The triggered subsystem was represented in blue color in Figure 114. The compression ratio, exhaust port locations, and net pressure rise were provided as an input to the triggered subsystem. With the variable displacement every cycle, the compression ratio, and exhaust port opening locations were also changed. Consequently, the 50\% MFB location was changed with every cycle. The third set of blocks consisted of a PID controller. This controller modified the ignition timing so that the $50 \%$ MFB occurred exactly at $0.24 \mathrm{~mm}$ after TDC. At first, the initial spark velocity was provided as an input to the numerical model. The spark velocity of $-4 \mathrm{~m} / \mathrm{s}$ was considered as an initial value for the ignition timing. This value was later scaled by the PID controller gradually for the maximized indicated thrust. The PID controller actions on 50\% MFB location and ignition timing are shown in Figure 115. 


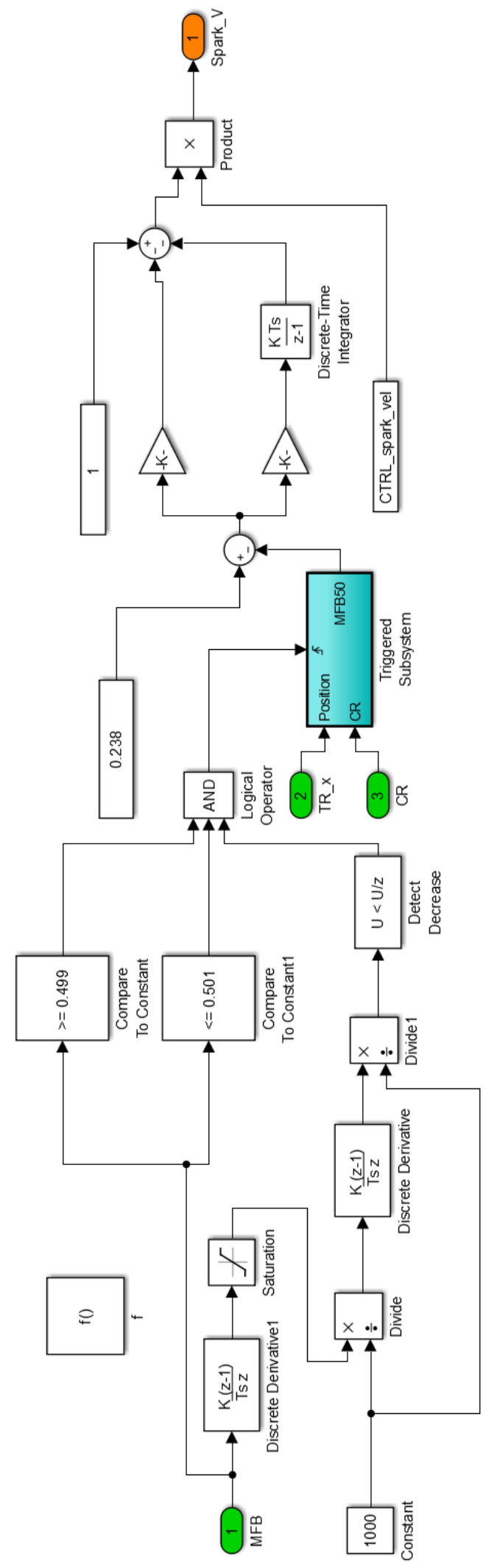

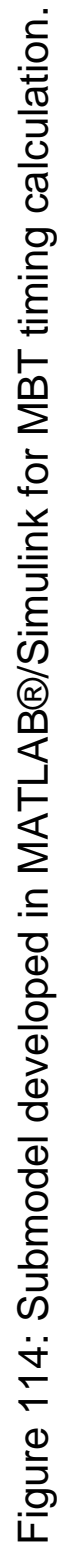


From Figure 115, initially in the generation phase, the ignition was activated almost at TDC. As a result, the compression ratio was increased initially. Moreover, the 50\% MFB location occurred at $7 \mathrm{~mm}$ after TDC. With the MBT algorithm, the ignition timing was optimized for the maximum indicated thrust. In other words, the ignition timing was changed gradually from $-1 \mathrm{~m} / \mathrm{s}$ to $-4.8 \mathrm{~m} / \mathrm{s}$. With the change in the ignition timing, the $50 \%$ MFB location was also changed from $7 \mathrm{~mm}$ to $0.24 \mathrm{~mm}$ after TDC. In summary, the current MBT algorithm eliminated the manual tuning process for an optimized FPEG performance.

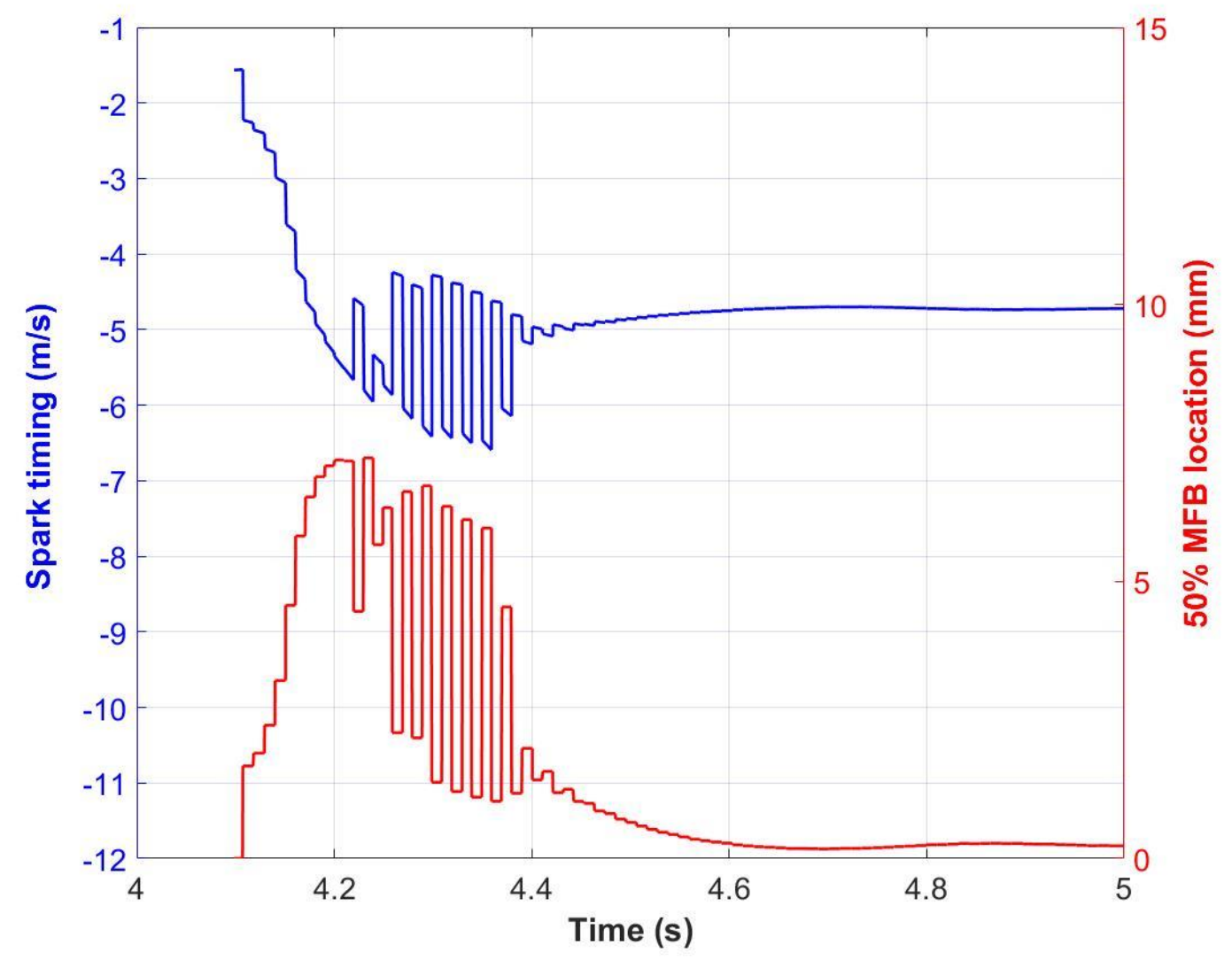

Figure 115: PID controller action in regulating the 50\% MBT location and spark timing.

After calculating the MBT location, the state flow diagram was used to activate the modeled combustion process. The combustion process activation in the model referred to the initiation of the HRR process. The state flow diagram is shown in Figure 116. The output of the state flow diagram was the binary spark signal. The binary output of ' 1 ' acted as a trigger to the combustion submodel. Before the generating mode, the ignition system was turned off. This was because of 
non-favorable combustion conditions in the engine cylinder. After entering the generation mode, by default, the ignition signal was turned off. During the compression stroke, the controller checked if the actual velocity was less than the MBT ignition velocity. If that condition was true, the ignition signal was changed from off state to hold state. Once the actual velocity exceeded the MBT velocity, the spark signal was generated. This spark signal was later provided as an input to the engine cylinder model. Finally, due to the spark signal, the combustion process was initiated according to the time-based Wiebe function.

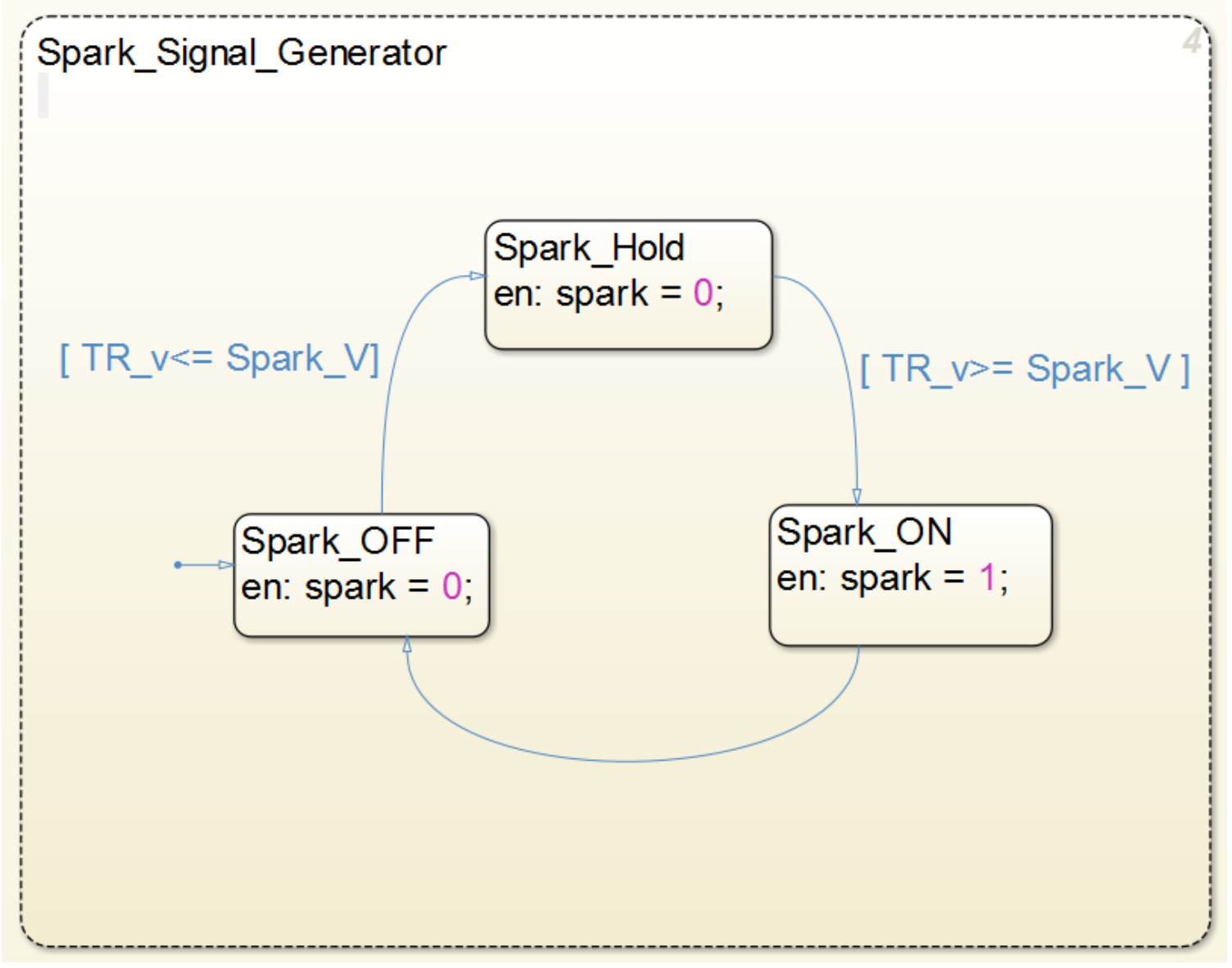

Figure 116: Submodel for spark signal generation in MATLABß/Simulink.

\subsection{Injection pulse width \& signal controller}

The fuel delivery into the FPEG system was modeled for both fumigated and direct-injected operation. In a fumigated operation, the fuel based on the mass flow controller was delivered into the intake throat volume. This intake throat volume in the model referred to the crankcase compression volume in the experimental FPEG system. At the SPO, the homogenous mixture 
was then transferred into the combustion chamber. This delivery was modeled by using a pressure gradient flow model.

In a direct-injected operation, the fuel was directly introduced into the combustion chamber. The air was delivered to the combustion chamber through the scavenging ports. The model considered only the homogenous mixture condition. In other words, the model failed to capture the mixing properties of fuel and air mixture. Thus, the fuel can be introduced either before or after the EPC. However, the mixture stratification issues prevent the fuel to be injected after the EPC for an injection pressure of 4.1 bar [117]. To mimic the experimental process, the fuel was injected around BDC. Unlike the flow models mentioned for exhaust and intake, the fuel injection flow was only one-directional. In other words, there was no backflow of fuel into the injector throttle area based on the pressure difference.

The fuel injection controller required the information of Start of Injection (SOI) and pulse width of the injector. Similar to the ignition timing, these values can be specified in terms of velocity or time or displacement. For example, the SOI can be defined to occur $0.1 \mathrm{~ms}$ after BDC or when the velocity exceeds $0.5 \mathrm{~m} / \mathrm{s}$ or when the displacement exceeds $5 \%$ of effective stroke from BDC. In an FPEG system, the displacement was changed from cycle-to-cycle. With the displacement changes, the dead center positions were also changed. To incorporate these cycle-to-cycle variations, the SOI was defined in terms of displacement. The control logic for the SOI was shown in Figure 117.

Initially, in the motoring mode, the fuel was not introduced into the cylinder. During the generating mode, by default, the injector was on a hold mode. In Figure 117, the fuel was injected from the injector exactly at BDC. The controller changed the injector state from hold to 'ON' condition exactly at BDC. This SOI was considered as an input variable that can be also changed depending upon the requirements. In other words, the SOI position can be matched with the respective experimental SOI location. The next step in the process was the calculation of the pulse width. The pulse width in this investigation was calculated by using three PID controllers. These controllers were based on trapped equivalence ratio, exhaust oxygen, and exhaust hydrocarbon percentages. The control loop with PID controllers is shown in Figure 118. For a stoichiometric equivalence ratio and with zero exhaust oxygen and hydrocarbon percentages, the three PID controllers scaled the injection pulse. An initial pulse width of $2.5 \mathrm{~ms}$ was also provided to the controller. 


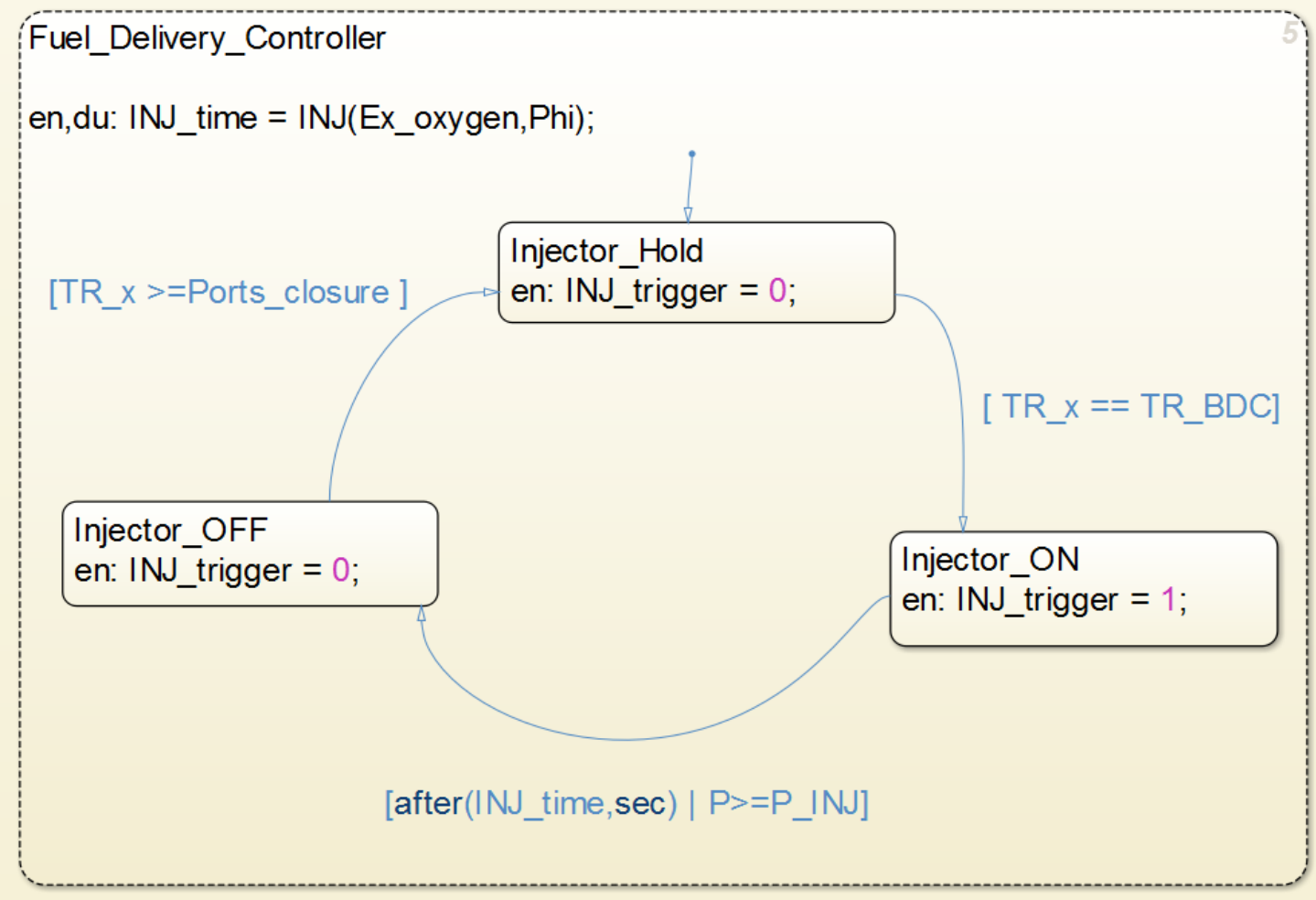

Figure 117: Generation of injection signals for an FPEG system at modeling level with varying displacement.

In addition, the direct injection must be completed in a defined, finite time window in an FPEG system. Experimentally, the fuel was injected into the engine cylinder at a pressure of $4.1 \mathrm{bar}$. If the cylinder pressure exceeded the fuel injection pressure, then the flow was halted. Moreover, the fuel and air mixtures were back flowed into the injector if the injector was open. These backflows should be considered in the numerical model in order to mimic the experimental injection system. Therefore, the pressure of 4.1 bar was regarded as a critical pressure. If the cylinder pressure during the injection process exceeded the critical injection pressure, the injector was turned off. The same control logic is shown in Figure 117 when the injector changed its state from 'ON' to 'OFF' condition. The injector was in 'ON' condition when either one of the following two conditions was true. The first condition was the actual injection duration less than the prescribed injection duration. The second condition was the cylinder pressure less than the critical injection pressure. If any of these two conditions were false, the injector was turned off by the controller. With these two conditions, the modeled system mimicked the experimental injection process. 


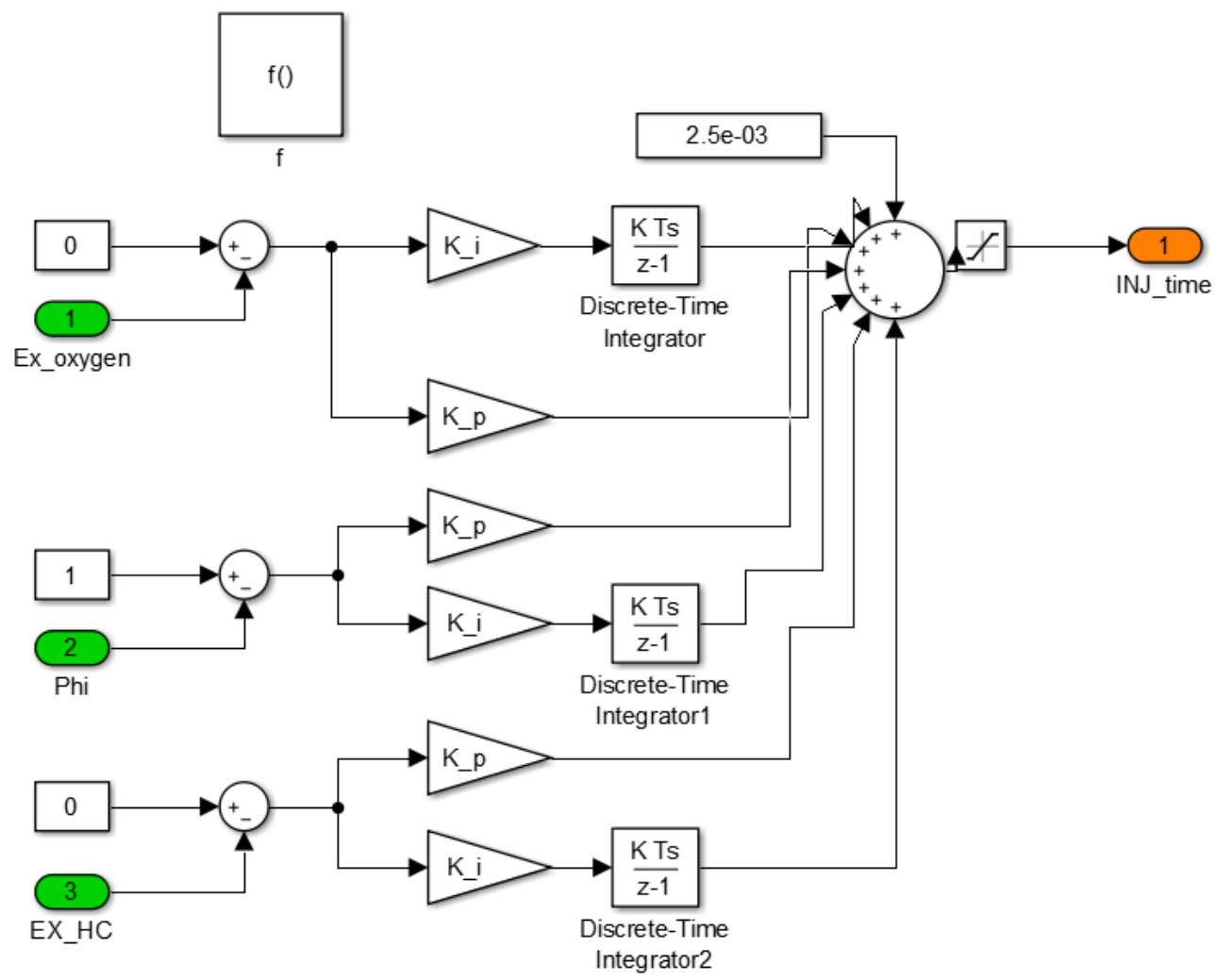

Figure 118: Injector pulse width calculation submodel based on feedback PID controllers.

Throughout this chapter, a fundamental multi-level cascade control strategy was explored for an FPEG system. The MATLAB® code generation capabilities allow the proposed controller to be integrated with the experimental system. Initially, a mode-switch controller was developed for the LEM. This mode-switch controller switched the LEM during the cases of cold-start, hot-start, stall, and misfired conditions. The numerical model was then expanded to develop a model-based calibration map controller. These calibrations acted as a supervisory controller for defining the FPEG operating conditions for a given load. Moreover, these calibrations improved the FPEG efficiency at part load conditions. This was due to the selection of operating conditions with high efficiency. Later, an energy management controller was developed for a stable FPEG operation. The feasibility of this energy management controller was checked for multiple control inputs. Finally, the control strategies were developed for MBT and injection timings for a stoichiometric FPEG operation. 


\section{CONCLUSIONS \& FUTURE WORK}

\subsection{Summary of Research Effort}

The main objective of this dissertation was to model spring-assisted FPEG dynamics and performance and to apply the modeling results for the experimental prototype development. In other words, the goal was to use the modeling results to optimize the experimental FPEG. In the final step, the experimental results were further utilized to improve the model processes and model understanding.

The FPEG system presented in this dissertation offered potential advantages over the CSE in terms of indicated efficiency. The design simplicity, friction reduction, and reduced moving parts improved the indicated efficiency of these systems over the CSE. However, the cycle-to-cycle variations and lack of robust system control resulted in an unstable FPEG operation. Mainly, the work presented in this dissertation contributed to the FPEG research in four aspects.

The first aspect of this dissertation was the accurate modeling and analysis of stiff spring-assisted FPEG system. To achieve this, a numerical model was developed in MATLAB®/Simulink for single and dual cylinder FPEG systems. The proposed model considered all the processes involved in the FPEG system. These processes include piston dynamics, combustion, heat transfer, thermodynamics, gas exchange, friction, motor, generator, and mechanical spring forces. Moreover, a component-based modeling procedure was implemented in this dissertation. This procedure provided a basis for future attempts that involve optimization of the experimental system. Finally, multiple model evaluations helped in understanding the operating characteristics and energy balance insights of FPEG systems. Moreover, these evaluations were ranged from weak or no spring to spring dominant systems. Finally, the FPEG characteristics were then compared with the CSE of the same size and frequency.

The second aspect of this dissertation validated the numerical model. Later, the experimental results were utilized to refine and improve the existing model. A $1 \mathrm{~kW}$, single cylinder FPEG system corresponding to a numerical model was developed at WVU. The experimental results obtained from the fumigated FPEG operation were used to validate the numerical model. The validation process was sought in two ways. The first way was the comparison of the FPEG system behavior during the starting mode. This way validated the LEM motor and spring models by 
excluding combustion, ignition, and heat transfer submodels. The second way considered the comparison of the FPEG system behavior during a stable generating mode. The coefficients used in various submodels were tuned to demonstrate the output accuracy. Moreover, a systematic strategy was devised and used to validate system behavior. Finally, the preliminary results from a direct-injected operation were provided to highlight FPEG cycle-to-cycle variations.

The third aspect of this dissertation was the model expansion for sensitivity analysis and parametric studies. These studies revealed the importance of several assumptions within the numerical model. Moreover, they also assisted in understanding the FPEG design space. In this dissertation, a Simulink based Sensitivity Analysis Toolbox ${ }^{\mathrm{TM}}$ was used for parametric and sensitivity studies. A total of 25 model inputs was identified and analyzed. The analysis helped in understanding the effects of these model inputs on FPEG system behavior. The results from these studies were illustrated with tornado charts and scatter plots. Finally, the conclusions from the sensitivity analysis studies supported the interdependency of model inputs. Moreover, these studies also provided the basis for the optimization of the experimental system.

The fourth and final aspect of this dissertation considered the development of a multi-level control strategy for stable FPEG operation. The proposed control strategy included controls for LEM mode switching, model-based calibration, energy management, ignition, and injection timings. At first, the LEM switching logic between motoring and generator modes was presented. In this logic, initially, the LEM was allowed to act as a motor until the combustion favorable conditions were reached. After attaining the combustion favorable conditions, the LEM was switched to the generating mode. Later, a model-based calibration map controller defined the target FPEG operating parameters for a given load demand. Despite the stiff spring addition, the FPEG needed an external controller for stable operation. As a result, an energy management controller was devised to control the LEM load for different control inputs. Finally, an MBT algorithm based on cylinder pressure was devised for a wide operating range. The combined use of all these controllers demonstrated the current state-of-art control. Moreover, these controllers extended the FPEG system usage for a wide LEM load demand.

\subsection{Major conclusions}

Based on the research effort and results presented in this dissertation, the following conclusions can be made. 
- Both in the experimental and modeled FPEG system, a high interdependency of variables was observed. This high interdependency was essential to define the basis when examining the effects of specific variables in an FPEG system.

- With stiff spring addition, the FPEG dynamics were nearly identical to the CSE dynamics when the ports were closed. However, the deviations in the dynamics were observed mainly when the ports were opened. These dynamics variations were mainly attributed to the deflection profile of stiff springs.

- The total gas exchange duration was reduced by $12.0 \%$ and $2.5 \%$ for dual and single FPEG systems when compared to the CSE. Especially, the scavenging duration alone was reduced by $14.8 \%$ and $5.7 \%$ for dual and single cylinder FPEG systems. These reduced gas exchange durations were responsible for the reduced fuel trapping inside the FPEG combustion chamber.

- With dynamics variations, the indicated efficiency of single and dual FPEG systems was $1.5 \%$ and $28.3 \%$ higher than the CSE for the same geometry and operating frequency. Moreover, a dual cylinder FPEG system had two engine cylinders of the same displacement used in the single cylinder design. A relatively high increase in the dual system efficiency was due to a reduction in the scavenging losses.

- In terms of electric power, the single and dual systems generated $827.5 \mathrm{~W}$ and $1752.7 \mathrm{~W}$ for an effective displacement of $34 \mathrm{cc}$, moving mass of $2.75 \mathrm{~kg}$, and spring stiffness of 558 $\mathrm{kN} / \mathrm{m}$. The dual system electric power was $112 \%$ higher than the single cylinder system electric power. For the same operating conditions and fuel input, the dual system power output should be twice the single system output. The additional $12 \%$ increase in the dual system electric power was because of high indicated power and frequency for the same conditions.

- The overall FPEG system efficiency was lower than the CSE system for the same operating conditions. The final efficiencies of single and dual FPEG systems were reduced by $9.1 \%$ and $14.1 \%$ when compared to the CSE. This efficiency reduction was due to the 
presence of additional losses in the FPEG systems. The losses included vibration, LEM, and inherent stiff spring losses.

- The stiff spring dominance on FPEG dynamics was demonstrated. By increasing the spring stiffness, the velocity profile became more sinusoidal with respect to time and symmetric with respect to the neutral location. Moreover, increasing the spring stiffness reduced the dynamics variations between the CSE and FPEG systems.

- For a total displacement of $34 \mathrm{cc}$, trapped compression ratio of 8.25 , and an assumed combustion efficiency of $95 \%$, the modeled frequency increased from $72.3 \mathrm{~Hz}$ to $80.8 \mathrm{~Hz}$ with increasing the stiffness from $372 \mathrm{kN} / \mathrm{m}$ to $744 \mathrm{kN} / \mathrm{m}$. For the same conditions, the electric power was increased from $812 \mathrm{~W}$ to $877 \mathrm{~W}$ for a single cylinder FPEG system. For the same conditions, in a dual cylinder system, the frequency was changed from 76.8 $\mathrm{Hz}$ to $84.1 \mathrm{~Hz}$. Moreover, the electric power was increased from $1710 \mathrm{~W}$ to $1840 \mathrm{~W}$ for the same conditions.

- For a total displacement of $34 \mathrm{cc}$, and an operating frequency of $80 \mathrm{~Hz}$, the total gas exchange duration was highest for the CSE and lowest for the dual cylinder FPEG system. This lowest gas exchange in the dual design was because of the presence of combustion forces from another engine cylinder at BDC. When compared to the CSE, the total gas exchange duration was reduced by $12.1 \%$ and $2.5 \%$ for dual and single FPEG systems. Similarly, the scavenging duration alone was reduced by $14.8 \%$ and $5.7 \%$ for dual and single cylinder FPEG systems respectively.

- An experimental FPEG system corresponding to the numerical model was developed. The experimental system generated $550 \mathrm{~W}$ of electricity with an indicated efficiency of $13.8 \%$. Later, the experimental results validated the relationships used in the numerical model.

$>$ The resonance starting method was found to be viable for starting the FPEG system. The feasibility was demonstrated by both the modeled and experimental systems. The experimental system reached EPO, SPO, and $20 \%$ of the maximum stroke in 21,39 , and 49 resonant cycles. To reach $67 \%$ of the maximum stroke, the stiff springs stored $27.5 \mathrm{~J}$ or $44.5 \%$ of the maximum spring energy storage. 
$>$ During the resonant starting process, the model predicted the displacement, velocity, and cylinder pressures with $92.3 \%, 90.5 \%$, and $89.0 \%$ accuracy when compared to the experimental resonance process.

$>$ During the generating process, the model predicted the displacement, velocity, cylinder pressure, and LEM power with $96.0 \%, 90.1 \%, 88.3 \%$, and $99.1 \%$ accuracies.

- The numerical model was further expanded for sensitivity analysis and parametric studies. These studies quantified the uncertainties created by the model inputs in predicting the model outputs. A total of 25 model inputs was considered ranging from geometry, engine, spring, and LEM parameters.

> Based on local and global sensitivity approaches, mainly seven inputs were found to have a dominant effect on FPEG performance. These inputs were the cylinder bore, effective stroke, scavenging pressure, exhaust pressure, spring stiffness, combustion duration, and compression ratio.

> A final global sensitivity analysis was carried for the main model inputs. The main inputs were selected based on the local and global sensitivity results. In the final sensitivity study, two different cases were investigated. These cases were variable and fixed stroke conditions. In the first case, the variable stroke length had a dominant effect on FPEG efficiency. This was followed by cylinder bore, compression ratio, and spring stiffness. The dominant effect of the stroke was due to an increase in the indicated work, trapped mixture, and velocities for the same frequency. The reduced dominance of the spring stiffness was due to the narrow frequency band. When the spring stiffness was increased from $486 \mathrm{kN} / \mathrm{m}$ to 702 $\mathrm{kN} / \mathrm{m}$, the frequency was increased by only $12 \mathrm{~Hz}$.

$>$ In the second case, the effective stroke length was maintained constant in order to mimic the experimental system. In this case, the frequency was found to have a dominant effect on the FPEG performance. This was followed by the compression ratio and cylinder bore. The reduced compression ratio and cylinder 
bore dominance was due to a reduction in the interactions induced by the stroke length.

- A multi-level control strategy was developed at the modeling level. The proposed control strategy included LEM mode switching, model-based calibration, energy management, ignition, and injection controls.

$>$ With the LEM mode switch controller, the resonance starting and generating behavior of the FPEG system was established. The feasibility of this LEM mode switch controller was demonstrated by the starting and generating characteristics used in the model validation.

Dith no energy management controller, the LEM consumed more or less energy than the combustion energy generated in one cycle. When the LEM consumed more energy than the combustion energy, the stroke, peak pressure, and velocity at mid-stroke were reduced on the following cycles. Later, the system stalled after certain cycles of operation if this process was continued.

When the combustion energy was more than the LEM demand, the stroke, cylinder pressure, and compression ratios were increased. At mid-stroke, this same combustion energy resulted in increased translator velocities. These parameters (compression ratio, stroke length, cylinder pressure, and velocity) were considered as control inputs for the energy management controller. In other words, these parameters defined the excess energy storage in the FPEG system.

> An active energy management controller was developed to match the engine output to the LEM demand. The feasibility of the developed controller was checked for four different inputs. The inputs were compression ratio, dead center clearance setpoints, peak cylinder pressure, and mid-stroke velocity. Of all the inputs, the dead center clearance setpoints, when used with stiff springs, reduced the cycleto-cycle variations to less than $5 \%$ in stroke length. The reduced cycle-to-cycle variations were mainly due to the combustion stability. This stability was attained because of the fixed port locations and similar conditions. 
An energy management controller based on mid-stroke velocity failed to control the FPEG system. In this case, the dead centers were deviated from their ideal locations. Moreover, the controller was underdefined. The compression ratio was changed from 5-7.5 over a span of 200 cycles. Due to these variations, the COV in the compression ratio was as high as $20 \%$.

> Finally, an MBT algorithm was proposed for the first time for the FPEG systems with variable stroke length. The proposed MBT algorithm was implemented on an FPEG system at the modeling level. Overall, the proposed controller with stiff springs reduced the modeled system's cycle-to-cycle variations to less than $5 \%$.

\subsection{Future work}

The research presented in this dissertation can be broadened in two ways in the future. The first way is the use of sensitivity analysis and model-based controller results in the optimization of the system design. The second way is to further improve the numerical model by using experimental results and sophisticated submodels.

Here are the few recommendations for improving the numerical model in the future:

- The most important future work related to this dissertation is to optimize the experimental system. This can be attained by extending the sensitivity analysis results.

- The next important work is to demonstrate the feasibility of the multi-level controller in the experimental system. The MATLAB® code generation capabilities make it easy to be integrated with the experimental system.

- The feasibility of the energy management controller should be tested experimentally. The procedure for implementing different energy management controllers is undergoing at the end of this dissertation. If successful, it allows for optimizing the FPEG combustion performance based on variable piston trajectories. 
- The proposed MBT timing should be implemented experimentally as a basis to control the combustion instabilities. Moreover, further improvements in the FPEG performance can be obtained by testing these controllers.

- Though not pursued in this investigation, it is possible to predict the FPEG performance by providing a reference sinusoidal signal to mimic the dynamics. It is also possible that the proposed controller can consider variable combustion parameters statistically based on the experimental system.

- For single and dual cylinder FPEG systems, the mount vibration and balancing issues are yet to be addressed [127]. Future research should examine implementing a vibrational model in the FPEG system. This model helps in understanding the impact of mounting on the FPEG performance. This problem can be easily addressed with confidence, by adding an additional spring-mass-damper in the numerical model.

- The implementation of linear and non-linear springs (bounce chamber) deserves attention. The parametric study should be focused on different spring designs to understand the systems stresses, spring inertia, frequency, and dynamics. Regardless, this parametric study enables different FPEG designs based on innovative spring design and variable motion.

- A more accurate and robust LEM model based on Finite Element Analysis (FEA) should be implemented [128]. This allows in understanding the effects of cogging, flux density, stator, and translator parameters on the FPEG performance.

- The complexity of the combustion and gas exchange processes was studied based on simplified mathematical models. The integration of one-dimensional or three-dimensional CFD models for these processes would surely help in improving the model accuracy. However, the model execution times may be increased substantially.

- Finally, the variant approach of the numerical model allows it to be adapted for a wider array of FPEG systems. These systems include but are not limited to different combustion modes, spring designs, LEM designs, and fuels. 


\section{BIBLIOGRAPHY}

[1] Atkinson, C. M., Petreanu, S., Clark, N. N., Atkinson, R. J., McDaniel, T. I., Nandkumar, S., and Famouri, P., "Numerical simulation of a two-stroke linear engine-alternator combination," SAE Technical Paper, no. 1999-01-0921, 1999. https://doi.org/10.4271/1999-01-0921

[2] Bade, M., Clark, N. N., Robinson, M. C., and Famouri, P., "Parametric Investigation of Combustion and Heat Transfer Characteristics of Oscillating Linear Engine Alternator," Journal of Combustion, vol. 2018, no. 2907562, 2018. https://doi.org/10.1155/2018/2907572

[3] Robinson, M. C., "Analysis and Optimization of a Dual Free Piston, Spring Assisted, Linear Engine Generator," Ph.D. Dissertation, West Virginia University, Morgantown, WV, USA, 2015. https://doi.org/10.33915/etd.6516

[4] Hung, N., and Lim, O., "A review of free-piston linear engines," Applied Energy, vol. 178, pp. 78-97, 2016. https://doi.org/10.1016/j.apenergy.2016.06.038

[5] Pateras, P. R., "Motor-compressor apparatus". United States of America Patent 1,657,641, 31 January 1928.

[6] Pateras, P. R., "Motor compressor of free piston type". United States of America Patent 2,241,957, 23 May 1941.

[7] Nuemann, K., "Junkers Free-Piston Compressor," Mechanical Engineering, vol. 57, no. 3, pp. 246-247, 1935.

[8] Farmer, H. O., "Free-piston compressor-engines," Proceedings of the Institution of Mechanical Engineers, vol. 156, no. 1, pp. 253-271, 1947.

https://doi.org/10.1243/PIME PROC $1947 \quad 156 \quad 043 \quad 02$

[9] London, A., and Oppenheim, A., "The free-piston engine development-Present status and design aspects," ASME Transactions, vol. 74, no. 2, pp. 1349-1361, 1952.

[10] Eichelberg, G., "Free piston generators," Swiss Construction Magazine, vol. 11, pp. 673-379, 1948.

[11] Braun, A., "Bounce chambers for multi-cylinder linear engine compressors". United States of America Patent 4,705,460, 10 November 1987. 
[12] Robinson, M. C., and Clark, N. N., "Fundamental Explorations of Spring-Varied, Free Piston Linear Engine Devices," Journal of Engineering for Gas Turbines and Power, vol. 137, no. 10, 2015. https://doi.org/10.1115/1.4030094

[13] Li, K., Zhang, C., and Sun, Z., "Precise piston trajectory control for a free piston engine," Control Engineering Practice, vol. 34, pp. 30-38, 2015.

https://doi.org/10.1016/j.conengprac.2014.09.016

[14] Toth-Nagy, C., "Linear engine development for series hybrid electric vehicles," Ph.D. Dissertation, West Virginia University, Morgantown, WV, USA, 2004. https://doi.org/10.33915/etd.2642

[15] Svrcek, M., and Edwards, C., "Emissions from an extreme-compression, free-piston engine with diesel-style combustion," International Journal of Engine Research, vol. 13, no. 3, pp. 238252, 2012. https://doi.org/10.1177/1468087411435207

[16] Callahan, T., and Ingram, S., "Free-piston engine linear generator for hybrid vehicles modeling study," Interim Report January - August 1994, Southwest Research Institute, San Antonio, TX, USA, 1995.https://apps.dtic.mil/dtic/tr/fulltext/u2/a294318.pdf

[17] Sandia National Laboratories, "Free-Piston Engines: A Possible Route to Hybrid-Electric Vehicles", Release date: $20^{\text {th }}$ November 2014, Last accessed: $27^{\text {th }}$ April 2020. http://crf.sandia.gov/free-piston-engines-a-possible-route-to-hybrid-electric-vehicles/

[18] Mikalsen, R., and Roskilly, A. P., "A review of free-piston engine history and applications," Applied Thermal Engineering, vol. 27, no. 14, pp. 2339-2352, 2007.

https://doi.org/10.1016/i.applthermaleng.2007.03.015

[19] Song, Y., Feng, H., Zuo, Z., Wang, M., and Guo, C., "Comparison research on different injection control strategy of $\mathrm{Cl}$ free piston linear generator in one-time starting process," Energy Procedia, vol. 61, pp. 1597-1601, 2014. https://doi.org/10.1016/..egypro.2014.12.180

[20] Wikipedia, “GM XP_500", Release date: $21^{\text {st }}$ March 2014, Last accessed: $27^{\text {th }}$ April 2020. https://de.wikipedia.org/wiki/GM XP-500.

[21] Kosaka, H., Akita, T., Moriya, K., Goto, S., Hotta, Y., Umeno, T., and Nakakita, K., "Development of free piston engine linear generator system part 1- Investigation of Fundamental Characteristics," SAE Technical Paper, no. 2014-01-1203, 2014.

https://doi.org/10.4271/2014-01-1203 
[22] Goertz, M., and Peng, L., "Free piston engine its application and optimization," SAE Technical Paper, no. 2000-01-0996, 2000. https://doi.org/10.4271/2000-01-0996

[23] Carter, D., and Wechner, E., "The free piston power pack: Sustainable power for hybrid electric vehicles," SAE Technical Paper, no. 2003-01-3277, 2003.

https://doi.org/10.4271/2003-01-3277

[24] Mikalsen, R., and Roskilly, A. P., "A computational study of free-piston diesel engine combustion," Applied Energy, vol. 86, no. 7, pp. 1136-1143, 2009.

https://doi.org/10.1016/i.apenergy.2008.08.004

[25] Xu, Z., and Chang, S., "Prototype testing and analysis of a novel internal combustion linear generator integrated power system," Applied Energy, vol. 87, no. 4, pp. 1342-1348, 2010. https://doi.org/10.1016/j.apenergy.2009.08.027

[26] Fredriksson, J., and Denbratt, I., "Simulation of a two-stroke free piston engine," SAE Technical Paper, no. 2004-01-1871, 2004. https://doi.org/10.4271/2004-01-1871

[27] Zhao, Z., Zhang, F., Huang, Y., and Zhao, C., "An experimental study of the cycle stability of hydraulic free-piston engines," Applied Thermal Engineering, vol. 54, no. 2, pp. 365-371, 2013. https://doi.org/10.1016/i.applthermaleng.2013.02.033

[28] Rahman, M. M., Ariffin, A. K., Jamaludin, N., and Haron, C., "Vibration fatigue analysis of cylinder head of a new two-stroke free piston engine using finite element approach," Structural Integrity and Durability, vol. 1, no. 2, pp. 121-129, 2005.

[29] Johansen, T. A., Egeland, O., Johannessen, E. A., and Kvamsdal, R., "Dynamics and control of a free-piston diesel engine," Journal of Dynamic Systems, Measurement, and Control, vol. 125, no. 3, pp. 468-474, 2003. https://doi.org/10.1115/1.1589035

[30] Golovitchev, V. I., Bergman, M., and Montorsi, L., "CFD modeling of diesel oil and DME performance in a two-stroke free piston engine," Combustion Science and Technology, vol. 179, no. 1, pp. 417-436, 2007. https://doi.org/10.1080/00102200600837242

[31] Van Blarigan, P., "Advanced internal combustion electrical generator," Proceedings of the 2002 DOE Hydrogen Program Review, Golden, CO, USA, 2002.

[32] Goldborough, S. S., and Van Blarigan, P., "Optimizing the scavenging system for a two-stroke cycle, free piston engine for high efficiency and low emissions: a computational approach," SAE Technical Paper, no. 2003-01-0001, 2003. https://doi.org/10.4271/2003-01-0001 
[33] Jia, B., Smallbone, A., Zuo, Z., Feng, H., and Roskilly, A. P., "Design and simulation of a twoor four-stroke free-piston engine generator for range extender applications," Energy Conversion and Management, vol. 111, pp. 289-298, 2016. https://doi.org/10.1016/j.enconman.2015.12.063

[34] Nandkumar, S., "Two-Stroke Linear Engine," M.S. Thesis, West Virginia University, Morgantown, WV, USA, 1998. https://doi.org/10.33915/etd.928

[35] Tikkasen, S., Lammila, M., Herranen, M., and Vilenius, M., "First cycles of the dual hydraulic free piston engine," SAE Technical Paper, no. 2000-01-2546, 2000.

https://doi.org/10.4271/2000-01-2546

[36] Wu, Y., Wang, Y., Zhen, X., Guan, S., and Wang, J., "Three-dimensional CFD (computational fluid dynamics) analysis of scavenging process in a two-stroke free-piston engine," Energy, vol. 68, pp. 167-173, 2014. https://doi.org/10.1016/j.energy.2014.02.107

[37] Johnson, T. A., Leick, M. T., and Moses, R. W., "Experimental Evaluation of a Prototype Free Piston Engine-Linear Alternator (FPLA) System," SAE Technical Paper, no. 2016-01-0677, 2016. https://doi.org/10.4271/2016-01-0677

[38] Gou, Y., "The performance simulation of single cylinder electric power confined piston engine," AIP Proceedings, vol. 1834, no. 1, 2017. https://doi.org/10.1063/1.4981579

[39] Mikalsen, R., and Roskilly, A. P., "The design and simulation of a two-stroke free-piston compression ignition engine for electrical power generation," Applied Thermal Engineering, vol. 28, no. 5, pp. 589-600, 2008. https://doi.org/10.1016/i.applthermaleng.2007.04.009

[40] Toth-Nagy. C., and Clark, N. N., "The linear engine in 2004," SAE Technical Paper, no. 200501-2140, 2005. https://doi.org/10.4271/2005-01-2140

[41] Schneider, S., and Friedrich, H. E., "Experimental Investigation and Analysis of Homogeneous Charge Compression Ignition in a Two-Stroke Free-Piston Engine," SAE International Journal of Engines, vol. 9, no. 2015-32-0706, pp. 365-373, 2016.

https://www.jstor.org/stable/26284819

[42] ARPA-e, "Oscillating Linear Engine Alternator", Release date: $18^{\text {th }}$ June 2015, Last accessed: $27^{\text {th }}$ April 2020. arpa-e.energy.gov/?q=slick-sheet-project/oscillating-linear-engine-and-alternator [43] Clark, N. N., Nandkumar, S., and Famouri, P., "Fundamental analysis of a linear two-cylinder internal combustion engine," SAE Technical Paper, no. 982692, 1998.

https://doi.org/10.4271/982692 
[44] Goldsborough, S. S., and Van Blarigan, P., "A numerical study of a free piston IC engine operating on homogeneous charge compression ignition combustion," SAE Technical Paper, no. 1999-01-0619, 1999. https://doi.org/10.4271/1999-01-0619

[45] Robinson, M. C., and Clark, N. N., "Study on the Use of springs in a Dual Free Piston Engine Alternator," SAE Technical Paper, no. 2016-01-2233, 2016. https://doi.org/10.4271/2016-01-2233

[46] Atchen, P. A. J., "A review of free piston engine concepts," SAE Technical Paper, no. 941776, 1994. https://doi.org/10.4271/941776

[47] Gong, X., Zaseck, K., Kolmanovsky, I., and Chen, H., "Dual-loop control of free piston engine generator," IFAC-Papers OnLine, vol. 48, no. 15, pp. 174-180, 2015.

https://doi.org/10.1016/i.ifacol.2015.10.025

[48] Zhu, Y., Wang, Y., Zhen, X., Guan, S., Wang, J., Wu, Y., Chen, Y., and Yin, S., "The control of an opposed hydraulic free piston engine," Applied Energy, vol. 126, pp. 213-220, 2014. https://doi.org/10.1016/j.apenergy.2014.04.007

[49] Hibi, A., and Ito, T., "Fundamental test results of a hydraulic free piston internal combustion engine," Proceedings of the Institution of Mechanical Engineers, Part D: Journal of Automobile Engineering, vol. 218, no. 10, pp. 1149-1157, 2004.

https://doi.org/10.1177/095440700421801010

[50] Bade, M., Clark, N., Famouri, P., and Guggilapu, P. D., "Translator dynamics and performance comparison on one and two cylinder free piston engines," ASME International Mechanical Engineering Congress \& Exposition, Pittsburgh, PA, USA, 2018.

[51] Bade, M., Clark, N. N., Famouri, P., and Guggilapu, P. D., "Performance Comparison of a Single Cylinder and a Dual Cylinder Free Piston Engine," Journal of Engineering for Gas Turbines and Power, vol. 141, no. 8, 2019. https://doi.org/10.1115/IMECE2018-88689

[52] Jeong, S., "Comparative Study of Linear Oscillating Generators," Ph.D. Dissertation, Technical University of Braunschweig, Braunschweig, Germany, 2015.

[53] Li, Q., Xiao, J., and Huang, Z., "Flat-type permanent magnet linear alternator: A suitable device for a free piston linear alternator," Journal of Zhejiang University, vol. 10, no. 3, pp. 345352, 2009. https://doi.org/10.1631/jzus.A0820224

[54] Rinderknecht, F., "A highly efficient energy converter for a hybrid vehicle concept-focused on the linear generator of the next generation," 2013 Eighth International Conference and Exhibition 
on Ecological Vehicles and Renewable Energies, Monte Carlo, Monaco, 2013. https://doi.org/10.1109/EVER.2013.6521533

[55] Leick, M. T., and Moses, R. W., "Experimental Evaluation of the Free Piston Engine-Linear Alternator (FPLA)," Technical Report, Sandia National Laboratories, Albuquerque, NM, USA, 2015.https://prod-ng.sandia.gov/techlib-noauth/access-control.cgi/2015/152095.pdf

[56] Petrichenko, D., Tatarnikov, A., and Papkin, I., "Approach to electromagnetic control of the extreme positions of a piston in a free piston generator," Modern Applied Science, vol. 9, no. 1, pp. 119-128, 2014. http://dx.doi.org/10.5539/mas.v9n1p119

[57] Oh, Y. I., Kim, G. C., and Lim, O. T., "A study for generating power on operating parameters of powerpack utilizng linear engine," Transactions of the Korean Hydrogen and New Energy Society, vol. 23, no. 2, pp. 183-190, 2012. https://doi.org/10.7316/KHNES.2012.23.2.183

[58] Jia, B., Zuo, Z., Feng, H., Tian, G., and Roskilly, A. P., "Development approach of a sparkignited free-piston engine generator," SAE Technical Paper, no. 2014-01-2894, 2014. https://doi.org/10.4271/2014-01-2894

[59] Petreanu, S., "Conceptual analysis of a four-stroke linear engine," Ph.D. Dissertation, West Virginia University, Morgantown, WV, USA, 2001. https://doi.org/10.33915/etd.2368

[60] Krasselt. J, Foster. D. E, Ghandhi. J, Herold. R, Reuss. D, and Najt. P, "Investigations into the Effects of Thermal and Compositional Stratification on $\mathrm{HCCl}$ Combustion - Part I: Metal Engine Results," SAE Technical Paper, no. 2009-01-1105, 2009.

https://doi.org/10.4271/2009-01-1105

[61] Xu, S., Wang, Y., Zhu, T., Xu, T., and Tao, C., "Numerical analysis of two-stroke free piston engine operating on $\mathrm{HCCl}$ combustion," Applied Energy, vol. 88, no. 11, pp. 3712-3725, 2011. https://doi.org/10.1016/j.apenergy.2011.05.002

[62] Chiang, C. J., Yang, J. L., Lan, S. Y., Shei, T. W., Chiang, W. S., and Chen, B. L., "Dynamic modeling of $\mathrm{SI} / \mathrm{HCCl}$ free-piston engine generators," 2011 6th IEEE Conference on Industrial Electronics and Applications, Beijing, China, 2011. https://doi.org/10.1109/ICIEA.2011.5975849

[63] Hung, N. B., Lim, O., and lida, N., "The effects of key parameters on the transition from SI combustion to $\mathrm{HCCl}$ combustion in a two-stroke free piston linear engine," Applied Energy, vol. 137, pp. 385-404, 2015. https://doi.org/10.1016/i.apenergy.2014.10.001 
[64] Van Blarigan, P., Pardiso, N., and Goldsborough, S. S., "Homogeneous charge compression ignition with a free piston: A new approach to ideal Otto cycle performance," SAE Technical Paper, no. 982484, 1998. https://doi.org/10.4271/982484

[65] Li, Q., Xiao, J., and Huang, Z., "Simulation of a two-stroke free-piston engine for electrical power generation," Energy \& Fuels, vol. 22, no. 5, pp. 3443-3449, 2008.

https://doi.org/10.1021/ef800217k

[66] Beachley, N. H., and Fronczak, F. J., "Design of a free-piston engine-pump," SAE Technical Paper, no. 921740, 1992. https://doi.org/10.4271/921740

[67] Toutant, W. T., "The Worthington-Junkers free piston air compressor," Naval Engineers Journal, vol. 64, no. 3, pp. 583-594, 1952.

https://doi.org/10.1111/j.1559-3584.1952.tb02985.x

[68] Edward, D., "Free piston gas generator". United States of America Patent 2,478,375, 9 August 1949.

[69] McMullen, J. J., and Ramsey, R., "The free-piston type of gas turbine plant and applications," ASME Transactions, vol. 76, no. 1, pp. 15-29, 1954.

[70] Specht, D. H., "Evaluation of free piston-gas turbine marine propulsion machinery in GTS William Patterson," SAE Technical Paper, no. 620280, 1962. https://doi.org/10.4271/620280

[71] Amann, C., "Evaluating alternative internal combustion engines: 1950-1975," Journal of Engineering for Gas Turbines and Power, vol. 121, no. 3, pp. 540-545, 1999.

https://doi.org/10.1115/1.2818506

[72] Atchen, P. A. J., and Vael, G. E. M., " The Innas Forklift Truck, Working under Constant Pressure," $1^{\text {st }}$ International Fluid Power Conference, Aachen, Germany, 1998.

[73] Famouri, P., Cawthorne, W. R., Clark, N., Nandkumar, S., Atkinson, C., Atkinson, R., McDaniel, T., and Petreanu, S., "Design and testing of a novel linear alternator and engine system for remote electrical power generation," IEEE Power Engineering Society 1999 Winter Meeting, New York, NY, USA, 1999. https://doi.org/10.1109/PESW.1999.747434

[74] Jia, B., Mikalsen, R., Smallbone, A., and Roskilly, A. P., "A study and comparison of frictional losses in free-piston engine and crankshaft engines," Applied Thermal Engineering, vol. 140, pp. 217-224, 2018. https://doi.org/10.1016/..applthermaleng.2018.05.018 
[75] Bade, M., Clark, N. N., Musho, T., and Famouri, P., "Piston rings friction comparison in a free piston engine and conventional crankshaft engines," ASME Internal Combustion Engine Fall Technical Conference, San Diego, CA, USA, 2018. https://doi.org/10.1115/ICEF2018-9774

[76] Flynn, G., "Observations on 25,000 hours of free-piston-engine operation," SAE Technical Paper, no. 570042, 1957. https://doi.org/10.4271/570042

[77] Yuan, C., Xu, J., and Feng, H., "In-cylinder heat transfer and gas motion of a free-piston diesel engine generator," Proceedings of the Institution of Mechanical Engineers, Part A: Journal of Power and Energy, vol. 231, no. 8, pp. 739-752, 2017. https://doi.org/10.1177/0957650917717627

[78] Aichlmayr, H. T., "Design considerations, modeling, and analysis of micro-homogeneous charge compression ignition combustion free-piston engines," Ph.D. Dissertation, Univeristy of Minnesota, Minneapolis, MN, USA, 2002.

http://citeseerx.ist.psu.edu/viewdoc/download?doi=10.1.1.467.9071\&rep=rep1\&type=pdf

[79] Jia, B., Zuo, Z., Feng, H., Tian, G., Smallbone, A., and Roskilly, A. P., "Effect of closed-loop controlled resonance based mechanism to start free piston engine generator: Simulation and test results," Applied Energy, vol. 164, pp. 532-539, 2016.

https://doi.org/10.1016/i.apenergy.2015.11.105

[80] Zulkifli, S. A., Karsiti, M. N., and Aziz, A. R. A., "Starting of a free-piston linear enginegenerator by mechanical resonance and rectangular current commutation," IEEE Vehicle Power and Propulsion Conference, Harbin, China, 2008. https://doi.org/10.1109/VPPC.2008.4677748

[81] Jia, B., Zuo, Z., Feng, H., Tian, H., and Roskilly, A. P., "Investigation of the starting process of free-piston engine generator by mechanical resonance," $6^{\text {th }}$ International Conference on Applied Energy, Taipei, China, 2014.

[82] Mikalsen, R., and Roskilly, A. P., "Performance simulation of a spark ignited free-piston engine generator," Applied Thermal Engineering, vol. 28, no. 14, pp. 1726-1733, 2008.

https://doi.org/10.1016/..applthermaleng.2007.11.015

[83] Baruah, P. C., "A free-piston engine hydraulic pump for an automotive propulsion system," SAE Technical Paper, no.880658, 1988. https://doi.org/10.4271/880658

[84] Huang, L., "An opposed-piston free-piston linear generator development for HEV," SAE Technical Paper, no. 2012-01-1021, 2012. https://doi.org/10.4271/2012-01-1021 
[85] Kleemann, A. P., Dabadie, J. C., and Henriot, S., "Computational design studies for a highefficiency and low-emissions free piston engine prototype," SAE Technical Paper, no. 2004-012928, 2004. https://doi.org/10.4271/2004-01-2928

[86] Mao, J., Zuo, Z., and Feng, H., "Parameters coupling designation of diesel free-piston linear alternator," Applied Energy, vol. 88, no. 12, pp. 4577-4589, 2011.

https://doi.org/10.1016/j.apenergy.2011.05.051

[87] Kaario, O., "Comparison between single-step and two-step chemistry in a compression ignition free piston engine," SAE Technical Paper, no. 2000-01-2937, 2000.

https://doi.org/10.4271/2000-01-2937

[88] Somhorst, J. H. E., and Achten, P. A. J., "The combustion process in a DI Diesel hydraulic free piston engine," SAE Technical Paper, no. 960032, 1996. https://doi.org/10.4271/960032

[89] Shoukry, E. F., "Numerical simulation for parametric study of a two-stroke compression ignition direct injection linear engine," Ph.D. Dissertation, West Virginia University, Morgantown, WV, USA, 2003. https://doi.org/10.33915/etd.1889

[90] Yuan, C., Feng, H., He, Y., and Xu, J., "Combustion characteristics analysis of a free-piston engine generator coupling with dynamic and scavenging," Energy, vol. 102, pp. 637-649, 2016. https://doi.org/10.1016/j.energy.2016.02.131

[91] Sun, C., Wang, Z., Yin, Z., and Zhang, T., "Investigation of control method for starting of linear internal combustion engine-linear generator integrated system," SAE Technical Paper, no. 201501-1729, 2015. https://doi.org/10.4271/2015-01-1729

[92] Yang, R., Gong, X., Hu, Y., and Chen, C., "Motion control of free piston engine generator based on LQR," 34th Chinese Control Conference, Hangzhou, China, 2015.

https://doi.org/10.1109/ChiCC.2015.7260927

[93] Nemecek, P., and Vysoky, O., "Control of two-stroke free-piston generator," 6th Asian Control Conference, Bali, Indonesia, 2006.

[94] Goto, S., Moriya, K., Kosaka, H., Akita, T., Hotta, Y., Umeno, T., and Nakakita, K., "Development of free piston engine linear generator system part 2-investigation of control system for generator," SAE Technical Paper, no. 2014-01-1193, 2014.

https://doi.org/10.4271/2014-01-1193 
[95] Mikalsen, R., and Roskilly, A. P., "The control of a free-piston engine generator. Part 2: Engine dynamics and piston motion control," Applied Energy, vol. 87, no. 4, pp. 1281-1287, 2010. https://doi.org/10.1016/j.apenergy.2009.06.035

[96] Zhang, C., and Sun, Z., "Trajectory-based combustion control for renewable fuels in free piston engines," Applied Energy, vol. 187, pp. 72-83, 2017.

https://doi.org/10.1016/j.apenergy.2016.11.045

[97] Zhang, C., Chen, F., Li, L., Xu, Z., Liu, L., Yang, G., Lian, H., and Tian, Y., "A Free-Piston Linear Generator Control Strategy for Improving Output Power," Energies, vol. 11, no. 1, 2018. https://doi.org/10.3390/en11010135

[98] Xu, Z., and Chang, S., "Improved moving coil electric machine for internal combustion linear generator," IEEE Transactions on Energy Conversion, vol. 25, no. 2, pp. 281-286, 2010. https://doi.org/10.1109/TEC.2009.2038368

[99] Xia, H., Pang, Y., and Grimble, M., "Hybrid modelling and control of a free-piston energy converter," 2006 IEEE Conference on Computer Aided Control System Design, Munich, Germany, 2006. https://doi.org/10.1109/CACSD-CCA-ISIC.2006.4776675

[100] Jia, B., Mikalsen, R., Smallbone, A., Zuo, Z., Feng, H., and Roskilly, A. P., "Piston motion control of a free-piston engine generator: A new approach using cascade control," Applied Energy, vol. 179, pp. 1166-1175, 2016. https://doi.org/10.1016/j.apenergy.2016.07.081

[101] Mao, J., Zuo, Z., Li, W., and Feng, H., "Multi-dimensional scavenging analysis of a freepiston linear alternator based on numerical simulation," Applied Energy, vol. 88, no. 4, pp. 11401152, 2011. https://doi.org/10.1016/i.apenergy.2010.10.003

[102] Sofianopoulos, A., Zhou, Y., Lawler, B., and Mamalis, S., "Gas exchange processes of a small HCCl free piston engine-A computational study," Applied Thermal Engineering, vol. 127, pp. 1582-1597, 2017. https://doi.org/10.1016/j.applthermaleng.2017.08.089

[103] Babajimopoulos, A., Assanis, D. N., and Fiveland, S. B., "An approach for modeling the effects of gas exchange processes on $\mathrm{HCCl}$ combustion and its application in evaluating variable valve timing control strategies," SAE Technical Paper, no. 2002-01-2829, 2002.

https://doi.org/10.4271/2002-01-2829 
[104] Meymian, N. Z., Clark, N. N., Musho, T., Darzi, M., Johsnson, D., and Famouri, P., "An optimization method for flexural bearing design for high-stroke high-frequency applications," Cryogenics, vol. 95, pp. 82-94, 2018. https://doi.org/10.1016/..cryogenics.2018.09.008

[105] Heywood, J., "Internal Combustion Engine Fundamentals," McGraw-Hill, Inc., New York, NY, USA, 1998.

[106] Blair, G., "Design and simulation of two-stroke engines," Society of Automotive Engineers, Warrendale, PA, USA, 1996.

[107] Wiebe, I., "Semi-empirical for the combustion speed," Proceedings of Conference on Piston Engines, Moscow, USSR, 1956.

[108] Darzi, M., Johnson, D., Ulishney, C., Bade, M., and Meymian, N. Z., "Baseline evaluation of ignition timing and compression ratio configurations on efficiency and combustion stability of a small-bore, two-stroke, natural gas engine," ASME 2017 International Mechanical Engineering Congress and Exposition, Tampa, FL, USA, 2017.https://doi.org/10.1115/IMECE2017-70078

[109] Ma, M. T., "Incorporation of lubricant shear-thinning in a two-dimensional lubrication analysis for automotive piston-ring packs," SAE Technical Paper, no. 2000-01-1786, 2000. https://doi.org/10.4271/2000-01-1786

[110] Woschni, G., "A universally applicable equation for the instantaneous heat transfer coefficient in the internal combustion engine," SAE Technical Paper, no. 670931, 1967.

https://doi.org/10.4271/670931

[111] Annand, W. J. D., "Heat transfer in the cylinders of reciprocating internal combustion engines," Proceedings of the Institution of Mechanical Engineers, vol. 177, no. 1, pp. 973-996, 1963. https://doi.org/10.1243/PIME PROC 196317706902

[112] Hohenberg, G. F., "Advanced approaches for heat transfer calculations," SAE Technical Paper, no. 790825, 1979. https://doi.org/10.4271/790825

[113] Patir, N., and Cheng, H. S., "An average flow model for determining effects of threedimensional roughness on partial hydrodynamic lubrication," Journal of Lubrication Technology, vol. 100, no. 1, pp. 12-17, 1978. https://doi.org/10.1115/1.3453103

[114] Greenwood, J. A., and Tripp, J. H., "The contact of two nominally flat rough surfaces," Proceedings of the Institution of Mechanical Engineers, vol. 185, no. 1, pp. 625-633, 1970. https://doi.org/10.1243/PIME PROC 197018506902 
[115] Crouch, R. F., and Cameron, A., "Viscosity-temperature equations for lubricants," Journal of Institute of Petroleum Technologists, vol. 47, pp. 307-313, 1961.

[116] Subramanian, J., Mahmudzadeh, F., Bade, M., and Famouri, P., "Simulation and Experimental Validation of Equivalent Circuit Parameters and Core Loss in a Tubular Permanent Magnet Linear Generator for Free Piston Engine applications," 2019 IEEE International Electric Machines \& Drives, Sandiego, CA, USA, 2019.https://doi.org/10.1109/IEMDC.2019.8785345

[117] Darzi, M., “A Framework for Energy Optimization of Small, Two-Stroke, Natural Gas Engines for Combined Heat and Power Applications," Ph.D. Dissertation, West Virginia University, Morgantown, WV, USA, 2019. https://doi.org/10.33915/etd.4019

[118] Yuan, C., Ren, H., and Xu, J., "Comparison of the gas exchange of a loop scavenged freepiston engine alternator and the conventional engine," Applied Thermal Engineering, vol. 172, pp. 638-349, 2017. https://doi.org/10.1016/j.applthermaleng.2017.08.059

[119] Darzi, M., Johnson, D., Ulishney, C., Bade, R. M. B., Meymian, N. Z., Thompson, G., Clark, N., and Famouri, P., "Quantification of Energy Pathways and Gas Exchange of a Small Port Injection SI Two-Stroke Natural Gas Engine Operating on Different Exhaust Configurations," SAE Technical Paper, no. 2018-01-1278, 2018.https://doi.org/10.4271/2018-01-1278

[120] Ekstrom, P., "A simulation toolbox for sensitivity analysis," M.S. Project Report, Faculty of Science and Technology, Uppsala University, Uppsala, Sweden, 2005.

[121] Sobol, I. M., "On the distribution of points in a cube and the approximate evaluation of integrals," USSR Computational Mathematics and Mathematical Physics, vol. 7, no. 4, pp. 86112, 1967. https://doi.org/10.1016/0041-5553(67)90144-9

[122] Mathworks, "Statistics and Machine Learning Toolbox User's guide," The MathWorks, Inc., Natick, MA, 2019. https://www.mathworks.com/help/pdf doc/stats/stats.pdf

[123] Ulishney, C. J., "Experimental Design, Testing, and Evaluation of Methods to Improve the Efficiency and Reduce Emissions from a Small Two-stroke Natural Gas Engine," M.S. Thesis, West Virginia University, Morgantown, WV, USA, 2018.

https://doi.org/10.33915/etd.3707

[124] Bade, M., Clark, N., Famouri, P., Guggilapu, P. D., Darzi, M., and Johnson, D., "Sensitivity Analysis and Control Methodology for Linear Engine Alternator," SAE International Journal of 
Advances and Current Practices in Mobility, vol.128, no. 2019-01-0230, pp. 578-587, 2019. https://doi.org/10.4271/2019-01-0230

[125] Bade, M., Clark, N., Famouri, P., and Guggilapu, P. D., "Feasibility of Multiple Piston Motion Control Approaches in a Free Piston Engine Generator," SAE Technical Paper, no. 2019-012599, 2019. https://doi.org/10.4271/2019-01-2599

[126] Zhu, G. G., Daniels, C. F., and Winkelman, J., "MBT timing detection and its closed-loop control using in-cylinder pressure signal," SAE Technical Paper, no. 2003-01-3266, 2003. https://doi.org/10.4271/2003-01-3266

[127] Meymian, N. Z., Clark, N., Subramanian, J., Hieskell, G., Johnson, D., Mahmudzadeh, F., Darzi, M., Musho, T., and Famouri, P., "Quantification of windage and vibrational losses in flexure springs of a one kW two-stroke free piston linear engine alternator," SAE Technical Paper, no. 2019-01-0816, 2019. https://doi.org/10.4271/2019-01-0816

[128] Subramanian, J., Hieskell, G., Mahmudzadeh, F., and Famouri, P., "Study of radial and axial magnets for linear alternator-Free piston engine system," 2017 North American Power Symposium (NAPS), Morgantown, WV, USA, 2017.

https://doi.org/10.1109/NAPS.2017.8107293 